\title{
Electrical Status Epilepticus in Sleep
}

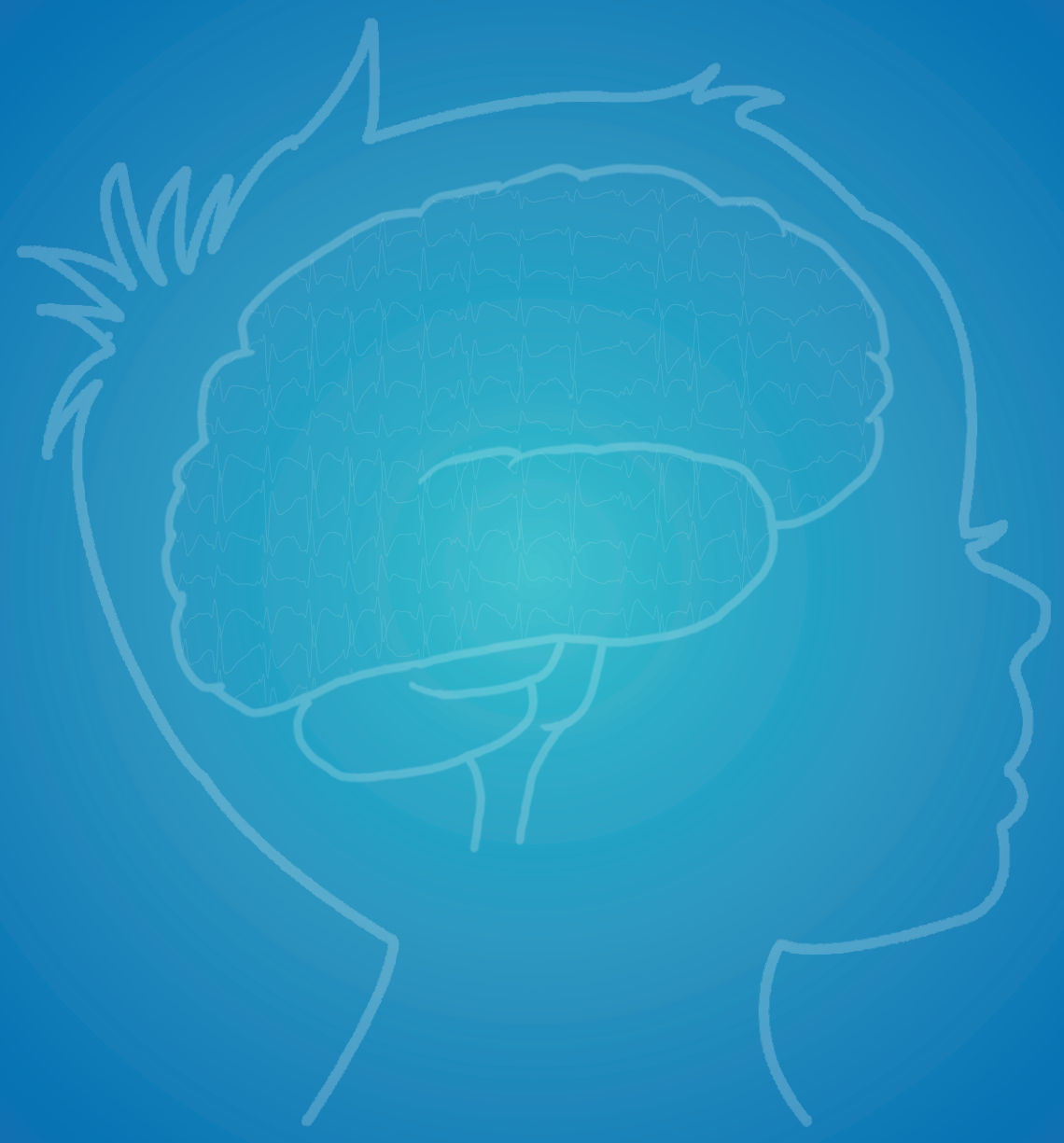

Bart van den Munckhof 


\section{Electrical Status \\ Epilepticus in Sleep}

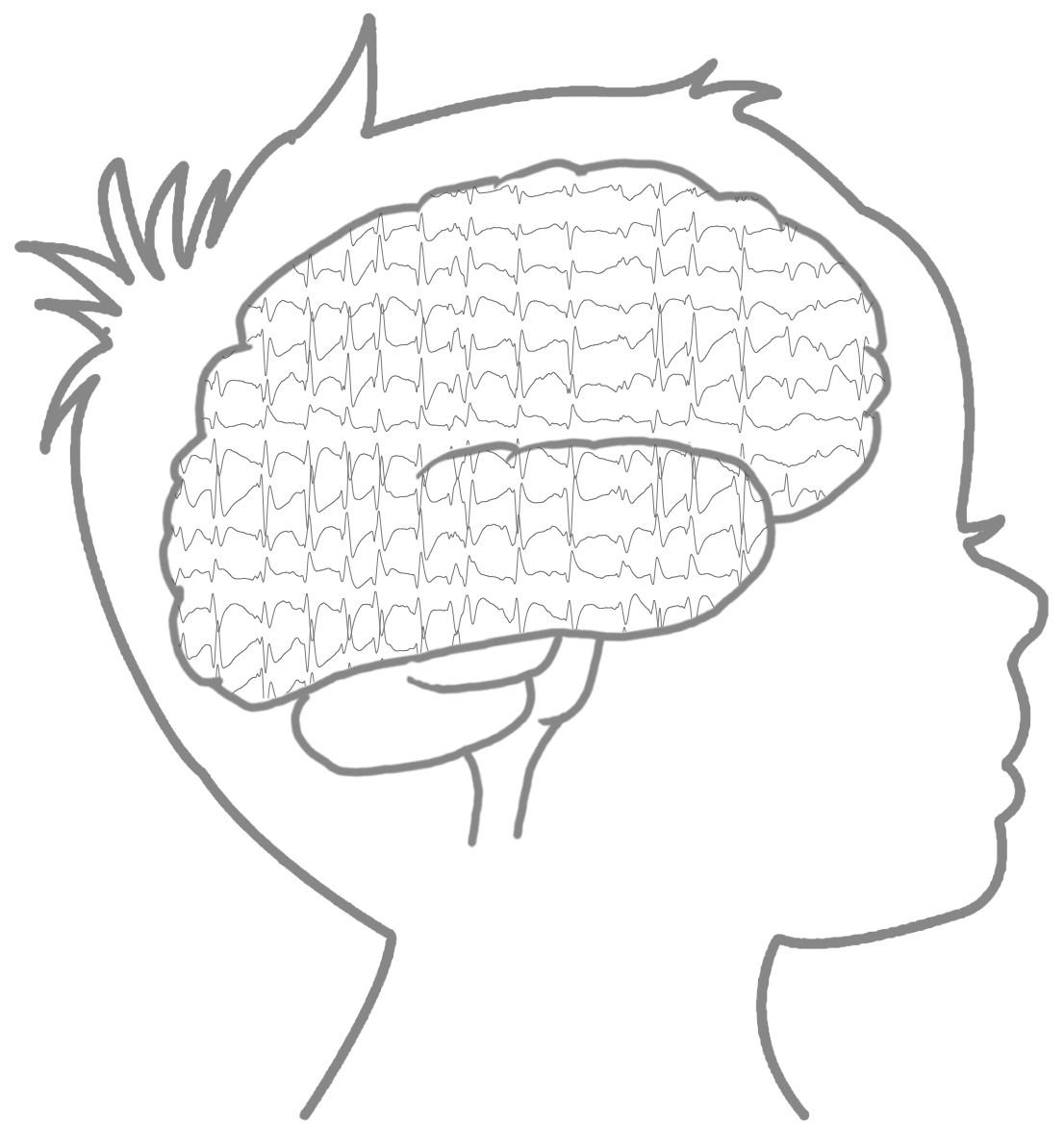

Bart van den Munckhof 
Copyright Bart van den Munckhof, 2020

ISBN: 978-94-93184-62-6

Layout and design: proefschrift AIO, Guus Gijben

Cover design: Sophie van Driel, adapted by Guus Gijben

Printed by: proefschrift-aio.nl

Financial support for the research presented in this thesis was generously provided by the Dutch Epilepsy Fund (Epilepsiefonds) and the Wilhelmina Children's Hospital research fund (WKZ fonds).

Logistical support for the conduct of the RESCUE ESES trial was provided by the European Clinical Research Infrastructures Network (ECRIN)

Financial support for the printing of this thesis by the UMC Utrecht Brain Center, UCB Pharma, Eisai B.V., ChipSoft and by Peter van den Munckhof and Annemiek Hamers is gratefully acknowledged. 


\section{Electrical Status Epilepticus in Sleep}

\section{Elektrische Status Epilepticus in Slaap}

(met een samenvatting in het Nederlands)

\section{Proefschrift}

ter verkrijging van de graad van doctor aan de

Universiteit Utrecht

op gezag van de

rector magnificus, prof.dr. H.R.B.M. Kummeling,

ingevolge het besluit van het college voor promoties

in het openbaar te verdedigen op

donderdag 15 oktober 2020 des middags te 4.15 uur

door

\section{Bart van den Munckhof}

geboren op 7 juli 1986

te Nijmegen 


\section{Promotor:}

Prof. dr. K.P.J. Braun

Copromotor:

Dr. F.E. Jansen 


\section{Contents}

1. General introduction, aims and outline 7

\section{PART I. Aetiology and Pathophysiology}

2. Perinatal thalamic injury: MRI predictors of electrical status epilepticus in sleep and long-term neurodevelopment

3. Inflammatory mediators in human epilepsy: A systematic review and meta-analysis

4. Serum inflammatory mediators correlate with disease activity in electrical status epilepticus in sleep (ESES) syndrome

5. Sleep slow wave homeostasis and cognitive functioning in children with electrical status epilepticus in sleep

\section{PART II. Treatment}

6. Treatment of electrical status epilepticus in sleep: A pooled analysis of 575 cases

7. Treatment of electrical status epilepticus in sleep: Clinical and EEG characteristics and response to 147 treatments in 47 patients

8. Corticosteroids or clobazam for ESES syndrome: a European, multicenter, randomized, controlled clinical trial (RESCUE ESES)

9. Summary

10. General discussion

\section{Appendix}

Nederlandstalige samenvatting | Summary in Dutch 214

Dankwoord | Acknowledgements 220

List of Publications 230

Curriculum Vitae 

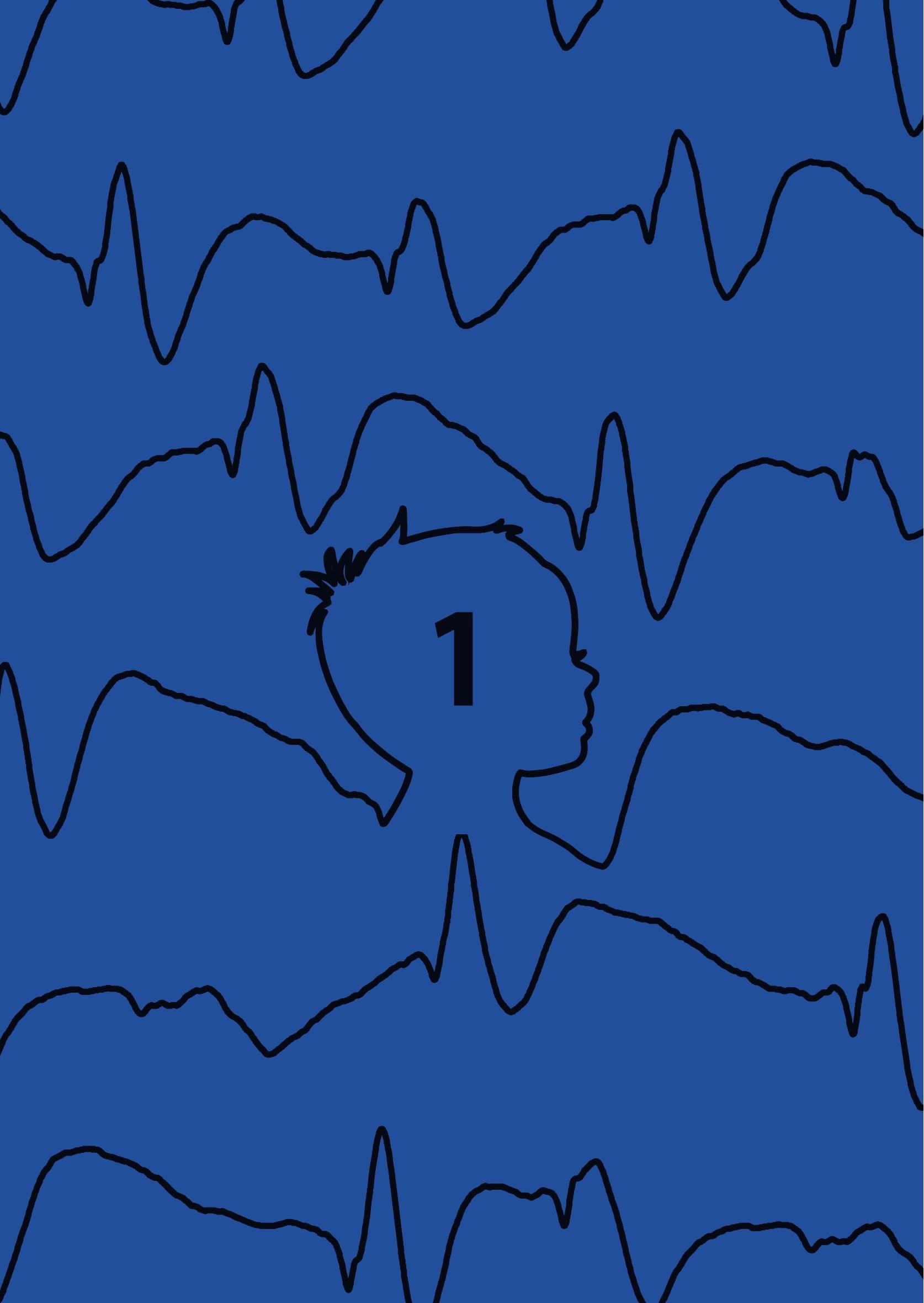


\section{Chapter 1}

General introduction 


\section{Historical perspective and terminology}

In 1971, electrical status epilepticus in sleep (ESES) was first described in six children with sleep induced continuous spike and wave discharges in the electro-encephalogram (EEG), that occurred night after night and sometimes lasting over a period of many years.' This dramatic EEG pattern persisted throughout the entire night and subsided upon awakening, but was not accompanied by usual manifestations of seizures during sleep. Most impressively, all of these children had cognitive deficits and five out of six also had clinical daytime seizures.

Variable terminology has been used since this first report; the combination of abundant epileptiform activity in sleep and cognitive deficits has been referred to as "encephalopathy with ESES", "ESES syndrome" or "continuous spikes and waves during (slow wave) sleep (CSWS)". The spike wave index (SWI), representing the proportion of sleep time with epileptiform activity, has been used to define the severity of epileptiform activity. Initially, a spike wave index above $85 \%$ was considered necessary for the diagnosis ESES, while more recently, cases with a SWI of 50-85\% are also considered part of the ESES spectrum. ${ }^{3.4}$ Epileptiform activity can be generalised or (multiffocal. Examples of sleep EEG findings in children with ESES are shown in figure 1.

\section{Epidemiology and clinical picture}

The exact incidence of encephalopathy with ESES is unknown; depending on study population and definition, it is estimated to constitute $0.2-1.9 \%$ of paediatric epilepsy cases. 5,6 Patients usually present with a delay, arrest or regression of development or behaviour, at an age between 2 and 12 years. Developmental deficits in children with ESES are diverse and include an acquired epileptic aphasia (Landau-Kleffner syndrome), global cognitive deficits, and pervasive developmental disorders. ${ }^{7-9}$ Some children are initially diagnosed with Rolandic epilepsy and later develop learning difficulties and ESES spectrum EEG-abnormalities. Others present with a developmental arrest or regression on top of a pre-existing delay. The majority (80-90\%) of the children with encephalopathy with ESES also have (occasional) seizures. ${ }^{6,10}$

\section{Aetiology and pathophysiology}

A cause for encephalopathy with ESES is found in a third up to half of the cases. Structural abnormalities, in particular thalamic injury and polymicrogyria (often accompanied by thalamic hypoplasia), have been linked to encephalopathy with ESES.11-13 Furthermore, children with mutations in the GRIN2A gene, encoding the a2 subunit of the N-methyl$\mathrm{d}$-aspartate (NMDA)-selective glutamate receptor, have been found to frequently develop ESES. ${ }^{14-16}$ Evidence that inflammation plays a role in patients with epilepsy, has accumulated over the years. ${ }^{17-21}$ In addition, immune proteins were found to also have a role in brain development and synaptic plasticity.2. More specifically, several findings suggest activation 
of the immune system in children with encephalopathy with ESES. In a previous study, antibrain antibodies were found in children with Landau-Kleffner Syndrome. ${ }^{23}$ Together with reported improvement in cognitive functioning after administration of corticosteroids ${ }^{24,25}$, this suggests that dysregulation of the immune system plays a role, at least in some ESES cases.

HowtheepileptiformEEGabnormalitiesinchildrenwithESES maylead to neurodevelopmental deficits, is incompletely understood. The so-called "synaptic homeostasis hypothesis" describes the process of daytime learning and nocturnal rest as a balance between the formation of new neuronal synapses (daytime synaptic upscaling) and the degradation of smaller synapses during sleep (nocturnal synaptic downscaling). ${ }^{26-28}$ Previous studies have suggested that synaptic downscaling during sleep -as reflected by slow wave morphology in sleep EEG recordings- is hampered in children with encephalopathy with ESES. ${ }^{29-31}$

\section{Treatment}

The evidence for the treatment of encephalopathy with ESES is limited to mostly small and retrospective studies. Uniform outcome measures to evaluate the effect of treatment are lacking. Conventional anti-epileptic drugs (AEDs) are often prescribed, even in children without overt clinical seizures, in an attempt to improve EEG findings and cognitive functioning in children with ESES. However, disappearance or reduction of the EEGabnormalities in sleep and cognitive improvement have been reported in only a minority of cases. ${ }^{32-34}$ Among conventional AEDs, sulthiam and levetiracetam have been reported to be relatively effective in ESES patients. ${ }^{34-37}$ Benzodiazepines are often considered the first-line treatment option in children with ESES and appear to be more effective than conventional AEDs in reducing the sleep-potentiated EEG-abnormalities and improving or stabilising cognition. ${ }^{34,38-40}$ Corticosteroids are often considered second-line treatment in the context of ESES with relatively high treatment success rates. ${ }^{24,25}$ In a subgroup of patients with a unilateral structural epileptogenic lesion, epilepsy surgery can be considered. ${ }^{41}$ Because of the limitations of previous studies, no definite recommendations can be made for the initial treatment of encephalopathy with ESES. A randomized controlled trial, with systematic assessment of neuropsychological and EEG outcomes, is needed to prove which first-line treatment is most effective.

\section{Prognosis}

ESES resolves spontaneously during puberty. However, several studies have shown that long-term neuropsychological outcome is variable and ranges from normal to severe intellectual disability. Patients with underlying structural abnormalities, earlier ESES onset, longer ESES duration and worse treatment response seem to have more severe cognitive sequelae. ${ }^{32,42,43}$ 


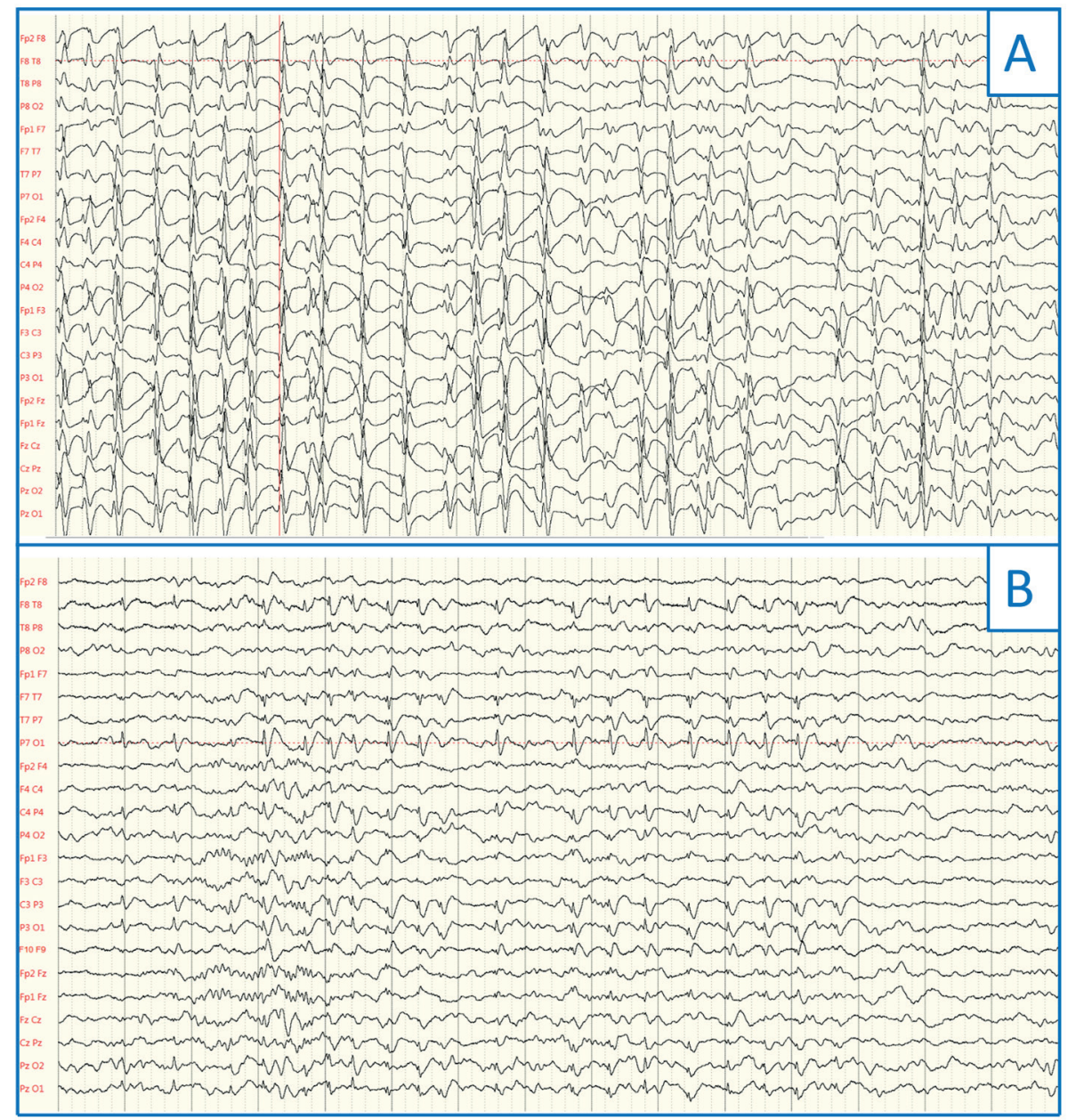

\section{Figure 1: Examples of EEG findings in children with encephalopathy with ESES}

A. EEG in non-REM sleep in a 10-year old girl with a past history of perinatal thalamic haemorrhage, who presented with a global developmental delay. The EEG shows bilateral synchronous diffuse high-amplitude spike-wave discharges and absence of physiological sleep phenomena ( $400 \mu \mathrm{V} / \mathrm{cm}, 15 \mathrm{sec} /$ page).

B. EEG in non-REM sleep in a 4 year old previously healthy boy, who presented with an acquired aphasia, showing bilateral (left-predominant) temporal spike-wave discharges and presence of normal sleep phenomena ( $200 \mu \mathrm{V} / \mathrm{cm}, 15 \mathrm{sec} /$ page).

\section{Outline of this thesis}

The main objectives of the studies described in this thesis are threefold: 1) to improve early recognition of children at risk of ESES development, 2) to increase our knowledge of ESES aetiology and pathophysiological mechanisms, and 3) to provide more evidence on optimal treatment regimens of children with encephalopathy with ESES (figure 2). 


\section{PART I. Aetiology and Pathophysiology}

In chapter 2, we studied, in a prospectively followed cohort, what proportion of patients with perinatal thalamic injury eventually developed ESES. Although previous studies have reported frequent co-occurrence, it was unknown how often patients with perinatal thalamic injury are affected. With this study, we also investigated whether neurodevelopmental outcome can be predicted with structural MRI volumetry of the thalamus, and whether diffusion tensor imaging characteristics are linked to neurodevelopment in children with perinatally acquired thalamic injury. In chapter $\mathbf{3}$, we report a systematic review and meta-analysis of inflammatory mediators, such as cytokines, in the broader context of patients with epilepsy. In chapter 4, we present the results of a pilot study of inflammatory mediators in blood samples of patients with encephalopathy with ESES.

In chapter 5, we investigated whether the extent of derangement of synaptic downscaling, as reflected by EEG slow waves, is associated with cognitive functioning (assessed with neuropsychological testing) and behavioural problems.

\section{PART II. Treatment}

In chapter 6 we describe a systematic review of the literature and pooled analysis of previous studies on the treatment of encephalopathy with electrical status epilepticus in sleep. In chapter 7, we assessed whether the effect of treatment on the EEG abnormalities is associated with subjective improvement in functioning and neuropsychological test results, and explored which factors predict treatment efficacy. In chapter 8 we present the study protocol of the European randomized controlled trial of treatment with corticosteroids versus clobazam (a benzodiazepine) in children with epileptic encephalopathy with ESES (acronym: RESCUE ESES*). The effect of treatment is evaluated with serial sleep EEG measurements and neuropsychological assessments over a period of 18 months and inflammatory mediators are analysed as a possible biomarker. We initiated and coordinated this international collaborative study with ongoing recruitment.

In chapter 9 the findings of the studies reported in this thesis are summarised. In chapter $\mathbf{1 0}$ the findings are interpreted in the context of existing knowledge, clinical implications are discussed, and suggestions for future research are proposed.

* RESCUE ESES: Randomized European trial of Steroids vs Clobazam Usage for Encephalopathy with Electrical Status Epilepticus in Sleep. 


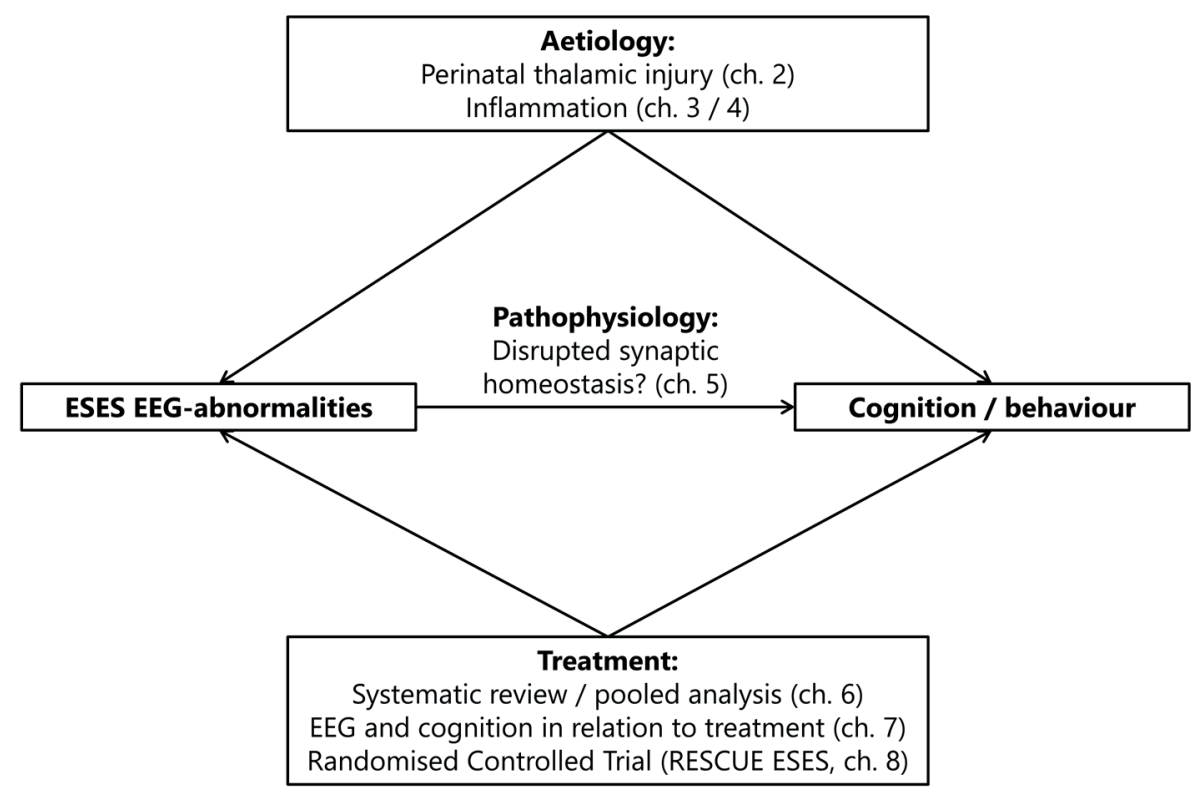

Figure 2: Schematic overview of the chapters of this thesis 


\section{References}

1. Patry G, Lyagoubi S, Tassinari CA. Subclinical "electrical status epilepticus"induced by sleep in children. Arch Neurol. 1971;24(3):242-252. doi:http://dx.doi.org/10.1001/archneur.1971.00480330070006

2. Fernández IS, Chapman KE, Peters JM, et al. The tower of Babel: Survey on concepts and terminology in electrical status epilepticus in sleep and continuous spikes and waves during sleep in North America. Epilepsia. 2013;54(4):741-750. doi:10.1111/epi.12039

3. Scheltens-De Boer M. Guidelines for EEG in encephalopathy related to ESES/CSWS in children. In: Epilepsia. Vol 50. ; 2009:13-17. doi:10.1111/j.1528-1167.2009.02211

4. Saltik S, Uluduz D, Cokar O, Demirbilek V, Dervent A. A clinical and EEG study on idiopathic partial epilepsies with evolution into ESES spectrum disorders. Epilepsia. 2005;46(4):524-533. doi:10.1111 /j.0013-9580.2005.45004

5. Hughes JR. A review of the relationships between Landau-Kleffner syndrome, electrical status epilepticus during sleep, and continuous spike-waves during sleep. Epilepsy Behav. 2011;20(2):247253. doi:10.1016/j.yebeh.2010.10.015

6. Sánchez Fernández I, Loddenkemper T, Peters JM, Kothare $S$ V. Electrical status epilepticus in sleep: Clinical presentation and pathophysiology. Pediatr Neurol. 2012;47(6):390-410. doi:10.1016/j. pediatrneurol.2012.06.016

7. Landau WM, Kleffner FR. Syndrome of acquired aphasia with convulsive disorder in children Neurology. 1957;7(8):1241, 8 pages following 1241. doi:10.1212/WNL.7.8.523

8. Giovanardi Rossi P, Parmeggiani A, Posar A, Scaduto MC, Chiodo S, Vatti G. Landau-Kleffner syndrome (LKS): long-term follow-up and links with electrical status epilepticus during sleep (ESES). Brain Dev. 1999;21(2):90-98. doi:10.1016/50387-7604(98)00071-0

9. Filippini M, Arzimanoglou A, Gobbi G. Neuropsychological approaches to epileptic encephalopathies. Epilepsia. 2013:54(SUPPL.8):38-44. doi:10.1111/epi.12422

10. Nickels K, Wirrell E. Electrical Status Epilepticus in Sleep. Semin Pediatr Neurol. 2008;15(2):50-60. doi:10.1016/j.spen.2008.03.002

11. Guzzetta F, Battaglia D, Veredice C, et al. Early thalamic injury associated with epilepsy and continuous spike-wave during slow sleep. Epilepsia. 2005;46(6):889-900. doi:10.1111/j.1528-1167.2005.64504

12. Kersbergen KJ, De Vries LS, Leijten FSS, et al. Neonatal thalamic hemorrhage is strongly associated with electrical status epilepticus in slow wave sleep. Epilepsia. 2013;54(4):733-740. doi:10.1111/ epi.12131

13. Bartolini E, Falchi M, Zellini F, et al. The syndrome of polymicrogyria, thalamic hypoplasia, and epilepsy with CSWS. Neurology. 2016;86(13):1250-1259. doi:10.1212/WNL.0000000000002526

14. Lemke JR, Lal D, Reinthaler EM, et al. Mutations in GRIN2A cause idiopathic focal epilepsy with rolandic spikes. NatPubl Gr. 2013;45(9):1067-1072. doi:10.1038/ng.2728

15. Lesca G, Rudolf G, Bruneau N, et al. GRIN2A mutations in acquired epileptic aphasia and related childhood focal epilepsies and encephalopathies with speech and language dysfunction. Nat Publ Gr. 2013:45(9):1061-1066. doi:10.1038/ng.2726

16. Carvill GL, Regan BM, Yendle SC, et al. GRIN2A mutations cause epilepsy-aphasia spectrum disorders. Nat Genet. 2013;45(9):1073-1076. doi:10.1038/ng.2727

17. Vezzani A, French J, Bartfai T, Baram TZ. The role of inflammation in epilepsy. Nat Rev Neurol. 2011;7(1):31-40. doi:10.2174/157339607782411538

18. Auvin S, Mazarati A, Shin D, Sankar R. Inflammation enhances epileptogenesis in the developing rat brain. Neurobiol Dis. 2010;40(1):303-310. doi:10.1016/j.nbd.2010.06.004

19. Kan $\mathrm{A}$ a, de Jager $\mathrm{W}$, de Wit $\mathrm{M}$, et al. Protein expression profiling of inflammatory mediators in human temporal lobe epilepsy reveals co-activation of multiple chemokines and cytokines. J Neuroinflammation. 2012;9(1):207. doi:10.1186/1742-2094-9-207 
Chapter 1. General introduction

20. Vezzani A, Moneta D, Richichi C, et al. Functional role of inflammatory cytokines and antiinflammatory molecules in seizures and epileptogenesis. Epilepsia. 2002;43 Suppl 5:30-35. doi:epis6012 [pii]

21. Vezzani A, Balosso S, Ravizza T. Neuroinflammatory pathways as treatment targets and biomarkers in epilepsy. Nat Rev Neurol. 2019. doi:10.1038/s41582-019-0217

22. Boulanger LM. Immune Proteins in Brain Development and Synaptic Plasticity. Neuron. 2009;64(1): 93-109. doi:10.1016/j.neuron.2009.09.001

23. Connolly AM, Chez MG, Pestronk A, Arnold ST, Mehta S, Deul RK. Serum autoantibodies to brain in Landau-Kleffner variant, autism, and other neurologic disorders. J Pediatr. 1999;134(5):607-613. doi:10.1016/S0022-3476(99)70248-9

24. Buzatu M, Bulteau C, Altuzarra C, Dulac O, Van Bogaert P. Corticosteroids as treatment of epileptic syndromes with continuous spike-waves during slow-wave sleep. Epilepsia. 2009;50:68-72. doi:10.1 111/j.1528-1167.2009.02224

25. Sinclair DB, SnyderTJ. Corticosteroids for theTreatment of Landau-Kleffner Syndrome and Continuous Spike- Wave Discharge During Sleep. 2005;32(5):300-306. doi:10.1016/j.pediatrneurol.2004.12.006

26. Huber R, Felice Ghilardi M, Massimini M, Tononi G. Local sleep and learning. Nature. 2004;430(6995): 78-81. doi:10.1038/nature02663

27. Tononi G, Cirelli C. Sleep function and synaptic homeostasis. Sleep Med Rev. 2006;10(1):49-62. doi:10.1016/j.smrv.2005.05.002

28. Tononi G, Cirelli C. Sleep and the Price of Plasticity: From Synaptic and Cellular Homeostasis to Memory Consolidation and Integration. Neuron. 2014;81(1):12-34. doi:10.1016/j.neuron.2013.12.025

29. Bölsterli BK, Schmitt B, Bast T, et al. Impaired slow wave sleep downscaling in encephalopathy with status epilepticus during sleep (ESES). Clin Neurophysiol. 2011;122(9):1779-1787. doi:10.1016/j. clinph.2011.01.053

30. Bölsterli Heinzle BK, Fattinger S, Kurth S, et al. Spike wave location and density disturb sleep slow waves in patients with CSWS (continuous spike waves during sleep). Epilepsia. 2014;55(4):584-591. doi:10.1111/epi.12576

31. Bölsterli BK, Gardella E, Pavlidis E, et al. Remission of encephalopathy with status epilepticus (ESES) during sleep renormalizes regulation of slow wave sleep. Epilepsia. 2017;58(11):1892-1901. doi:10.1111/epi.13910

32. Liukkonen E, Kantola-Sorsa E, Paetau R, Gaily E, Peltola M, Granström ML. Long-term outcome of 32 children with encephalopathy with status epilepticus during sleep, or ESES syndrome. Epilepsia. 2010. doi:10.1111/j.1528-1167.2010.02578

33. Caraballo RH, Veggiotti P, Kaltenmeier MC, et al. Encephalopathy with status epilepticus during sleep or continuous spikes and waves during slow sleep syndrome: A multicenter, long-term follow-up study of 117 patients. Epilepsy Res. 2013;105(1-2):164-173. doi:10.1016/j.eplepsyres.2013.02.010

34. Kramer U, Sagi L, Goldberg-Stern H, Zelnik N, Nissenkorn A, Ben-Zeev B. Clinical spectrum and medical treatment of children with electrical status epilepticus in sleep (ESES). Epilepsia. 2009;50(6):15171524. doi:10.1111/j.1528-1167.2008.01891

35. Fejerman N, Caraballo R, Cersõsimo R, Ferraro SM, Galicchio S, Amartino H. Sulthiame add-on therapy in children with focal epilepsies associated with encephalopathy related to electrical status epilepticus during slow sleep (ESES). Epilepsia. 2012;53(7):1156-1161. doi:10.1111/j.1528-1167.2012.03458

36. Aeby A, Poznanski N, Verheulpen D, Wetzburger C, Van Bogaert P. Levetiracetam efficacy in epileptic syndromes with continuous spikes and waves during slow sleep: Experience in 12 cases. Epilepsia. 2005. doi:10.1111/j.1528-1167.2005.00337

37. Larsson PG, Bakke KA, Bjørnæs $\mathrm{H}$, et al. The effect of levetiracetam on focal nocturnal epileptiform activity during sleep - A placebo-controlled double-blind cross-over study. Epilepsy Behav. 2012. doi:10.1016/j.yebeh.2012.02.024 
38. Sánchez Fernández I, Chapman K, Peters JM, et al. Treatment for continuous spikes and waves during sleep (CSWS): Survey on treatment choices in North America. Epilepsia. 2014;55:1099-1108. doi:10.1111/epi.12678

39. Sánchez Fernández I, Hadjiloizou S, Eksioglu Y, et al. Short-term response of sleep-potentiated spiking to high-dose diazepam in electric status epilepticus during sleep. Pediatr Neurol. 2012. doi:10.1016/j.pediatrneurol.2012.02.017

40. Veggiotti P, Pera MC, Teutonico F, Brazzo D, Balottin U, Tassinari CA. Therapy of encephalopathy with status epilepticus during sleep (ESES/CSWS syndrome): An update. Epileptic Disord. 2012;14(1):1-11. doi:10.1684/epd.2012.0482

41. Loddenkemper T, Cosmo G, Kotagal P, et al. Epilepsy surgery in children with electrical status epilepticus in sleep. Neurosurgery. 2009. doi:10.1227/01.NEU.0000336767.14252.76

42. Seegmüller C, Deonna T, Mayor Dubois C, et al. Long-term outcome after cognitive and behavioral regression in nonlesional epilepsy with continuous spike-waves during slow-wave sleep. Epilepsia. 2012;53(6):1067-1076. doi:10.1111/j.1528-1167.2012.03465

43. Pera MC, Brazzo D, Altieri N, Balottin U, Veggiotti P. Long-term evolution of neuropsychological competences in encephalopathy with status epilepticus during sleep: A variable prognosis. Epilepsia. 2013;54(SUPPL.7):77-85. doi:10.1111/epi.12313 


\section{PART I. \\ Aetiology and Pathophysiology}

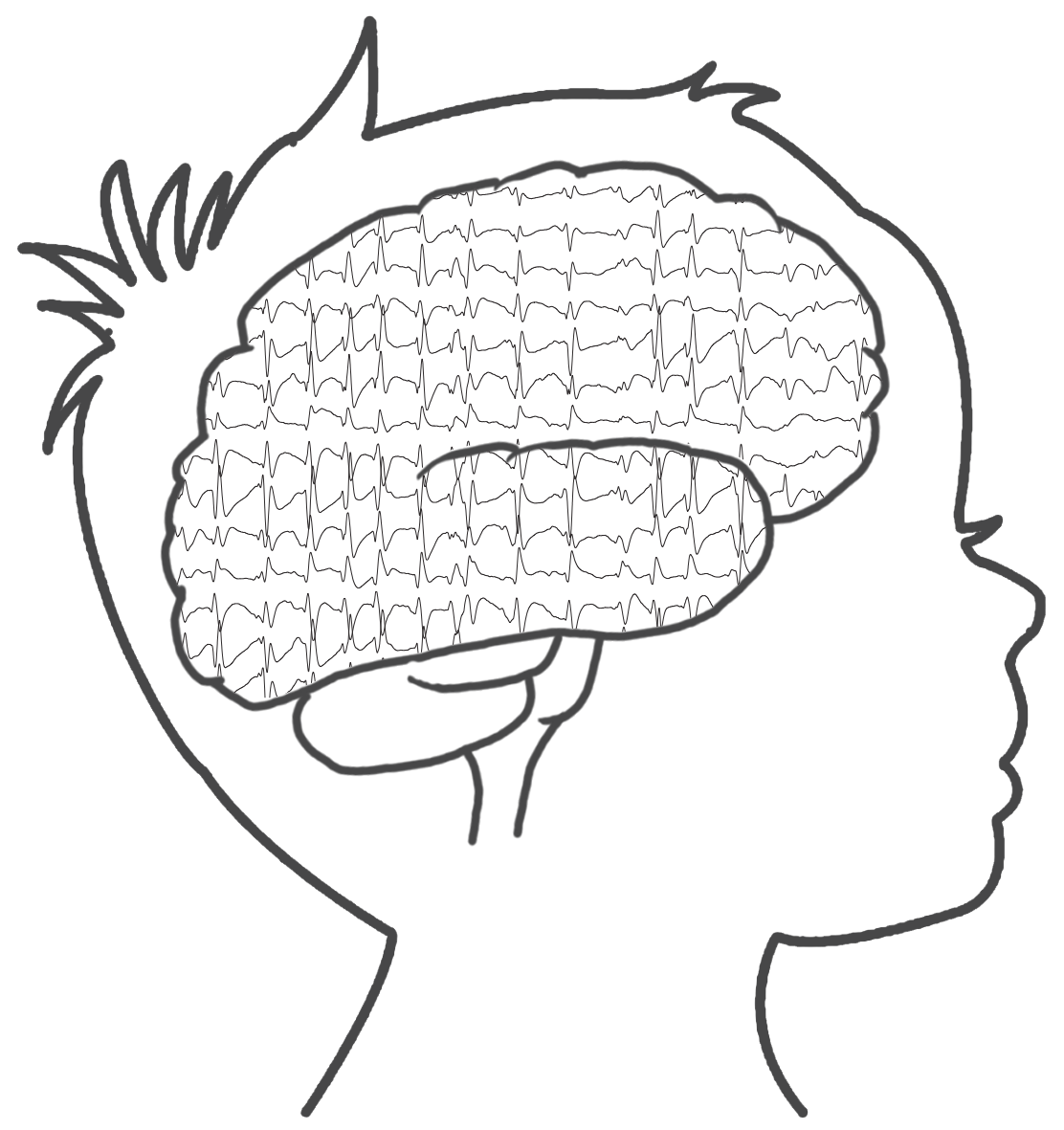



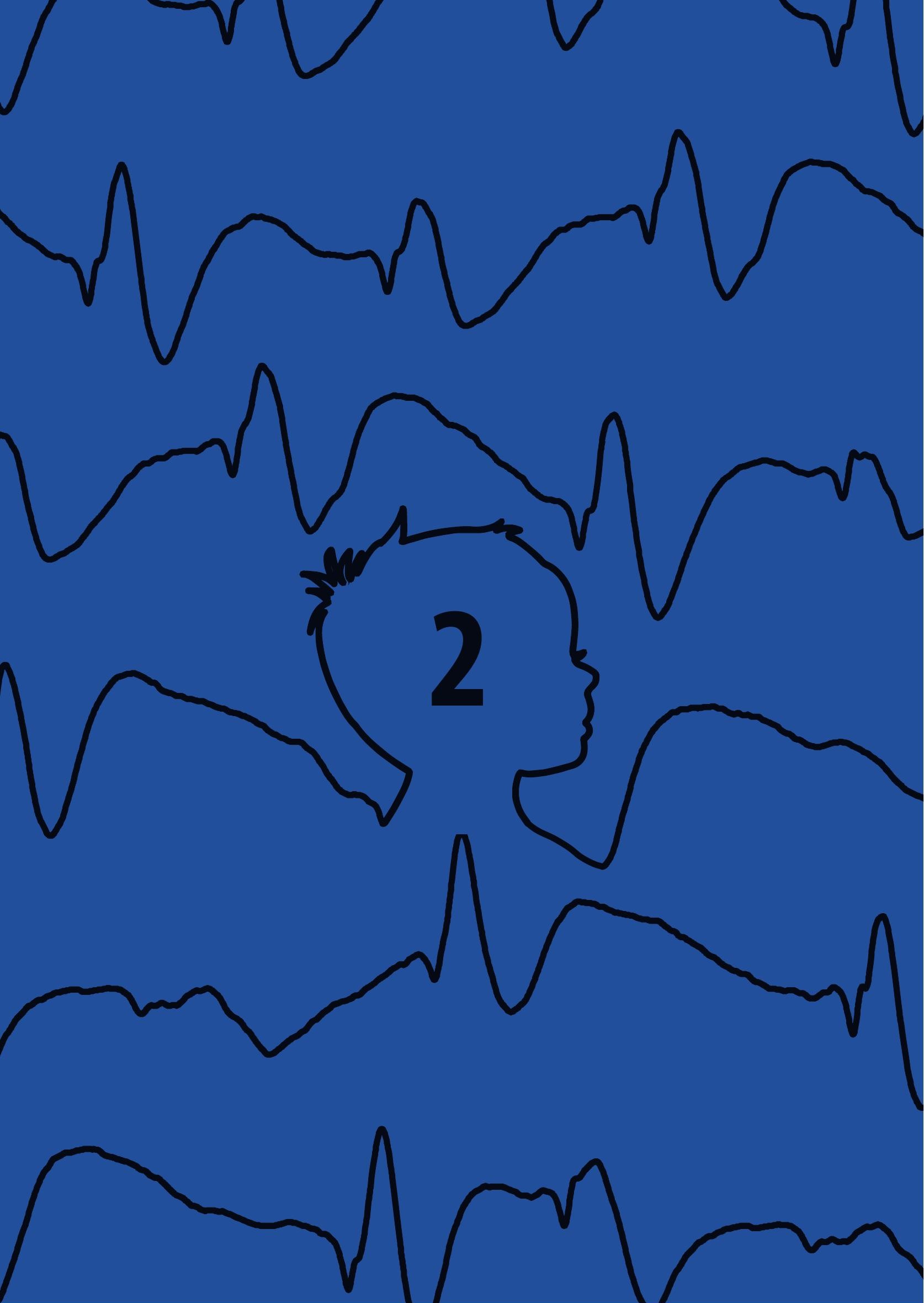


\section{Chapter 2}

\section{Perinatal thalamic injury: MRI predictors of electrical status epilepticus in sleep and long- term neurodevelopment}

\author{
Bart van den Munckhof \\ Anne F. Zwart \\ Lauren C. Weeke \\ Nathalie H.P. Claessens \\ Joost D.J. Plate \\ Alexander Leemans \\ Hugo J. Kuijf
}

\author{
Heleen C. van Teeseling \\ Frans S.S. Leijten \\ Manon J.N. Benders \\ Kees P.J. Braun \\ Linda S. de Vries \\ Floor E. Jansen
}




\section{Abstract}

Objective: Perinatal thalamic injury is associated with epilepsy with electrical status epilepticus in sleep (ESES). The aim of this study was to prospectively quantify the risk of ESES and to assess neuroimaging predictors of neurodevelopment.

Methods: We included patients with perinatal thalamic injury. MRI scans were obtained in the neonatal period, around three months of age and during childhood. Thalamic and total brain volumes were obtained from the three months MRI. Diffusion characteristics were assessed. Sleep EEGs distinguished patients into ESES (spike-wave index (SWI) $>85 \%$ ), ESES-spectrum (SWI 50-85\%) or no ESES (SWI<50\%). Serial Intelligence Quotient (IQ) / Developmental Quotient (DQ) scores were obtained during follow-up. Imaging and EEG findings were correlated to neurodevelopmental outcome.

Results: Thirty patients were included. Mean thalamic volume at three months was 8.11 $( \pm 1.67) \mathrm{ml}$ and mean total brain volume $526.45( \pm 88.99) \mathrm{ml}$. In the prospective cohort ( $n=23) 19$ patients (83\%) developed ESES (-spectrum) abnormalities after a mean followup of 96 months. In the univariate analysis, larger thalamic volume, larger total brain volume and lower SWI correlated with higher mean IQ / DQ after 2 years (Pearson's $r=0.74$, $p=0.001$; Pearson's $r=0.64, p=0.005$; and Spearman's rho $-0.44, p=0.03$ ). In a multivariable mixed model analysis, thalamic volume was a significant predictor of IQ / DQ (coefficient 9.60 [p<0.001], i.e., corrected for total brain volume and SWI and accounting for repeated measures within patients, a $1 \mathrm{ml}$ higher thalamic volume was associated with a 9.6 points higher IQ). Diffusion characteristics during childhood correlated with IQ / DQ after 2 years.

Significance: Perinatal thalamic injury is followed by electrical status epilepticus in sleep in the majority of patients. Thalamic volume and diffusion characteristics correlate to neurodevelopmental outcome.

\section{Key points box}

- The majority of patients with perinatal thalamic injury, will develop epilepsy with ESES after the age of two years.

- ESES is associated with neurodevelopmental deficits.

- Larger residual thalamic volume at the three months MRI correlates to higher total IQ / DQ during follow-up.

- MRI at three months appears to be useful to predict neurodevelopmental outcome in this population. 


\section{Introduction}

Perinatal thalamic injury can be the consequence of hemorrhage, ischemia or infection. Initial presentation in the neonatal period is often with lethargy and seizures. Cranial ultrasound generally reveals signs of thalamic injury and MRI is used to confirm the diagnosis and assess possible etiologies. Long-term neurodevelopmental outcome varies from normal to severe intellectual disability. ${ }^{1-5}$ After a seizure-free interval, epilepsy often recurs in childhood. Several studies have shown an association between perinatal thalamic injury and epilepsy with electrical status epilepticus in sleep (ESES). 1,2,6 How perinatal thalamic injury may cause ESES is unclear. Several studies suggested that loss of physiological thalamocortical interaction leads to pathological synchronized activity in NREM-sleep as well as loss of control over the developing cortico-cortical network. ${ }^{7-9}$

Epileptic encephalopathy with ESES is characterized by sleep-induced epileptiform activity accompanied by acquired neurodevelopmental deficits in children over 2 years of age..$^{10,11}$ Their sleep-EEG shows abundant epileptic discharges with a spike-wave index (SWI) of at least 50\%. ${ }^{12}$ ESES resolves spontaneously during puberty, while the developmental deficits generally persist afterwards. Early detection and prompt treatment initiation may improve neurodevelopmental outcome..$^{13,14}$

To the best of our knowledge it is not yet known, what proportion of patients with perinatal thalamic injury will develop epilepsy with ESES. We were able to address this question because these children are routinely in the follow-up clinic by a neonatologist and have interval EEGs in our center. In addition, early biomarkers for long-term neurodevelopment after perinatal injury were lacking and we aimed to study whether MRI results at three months and thalamic diffusion characteristics can predict neuropsychological assessment test results.

The aims of the current study were to: 1. quantify the proportion of children with perinatal thalamic injury that eventually develop epilepsy with ESES and ESES-spectrum in a prospectively followed cohort, 2. identify imaging predictors for the occurrence of ESES(spectrum) and for the severity of neurodevelopmental abnormalities. 3. explore whether DTI characteristics may reveal a possible mechanism that explains the occurrence of ESES and neurodevelopmental deficits after thalamic injury. 


\section{Methods}

\section{Patient selection}

We included patients with MRI-documented perinatal thalamic injury who were followed until at least 2 years of age and underwent at least one sleep EEG recording. Some of the included patients were also reported in a previous study. ${ }^{2}$

Subgroups were defined for the purpose of answering different research questions:

1) A prospectively followed cohort of consecutive patients who were diagnosed with perinatal thalamic injury between 2000 and 2015 and followed in our center from the neonatal period onwards. As part of routine clinical care in our hospital, these children undergo repeated screening of cognitive functioning by a neonatologist or pediatric neurologist, and undergo at least one sleep EEG after the age of two years. MRI scans are made in all children during the neonatal period, at around 3 months of age, and in the majority once again in childhood. Although the patients were not prospectively included in this study at the time of diagnosis of thalamic injury, they were prospectively followed according to our clinical protocol. This cohort was used to quantify the proportion of children with perinatal thalamic injury that developed epilepsy with ESES during follow-up.

2) Patients who were referred to our center during childhood with a history of perinatal thalamic injury - some of whom were referred because of epilepsy or ESES, diagnosed elsewhere. These patients were only included in this study if MRIs and EEG follow-up were available. This group of patients was added to the first cohort to correlate imaging findings from the neonatal period with neurodevelopment during follow-up. As they were not prospectively followed in our center, incidence of ESES could not be assessed in these patients.

\section{Data collection}

Clinical characteristics were collected from the neonatal period (gestational age, pregnancy / delivery complications, Apgar scores, etiology of injury, presence of seizures) and during follow-up (presence of seizures, neurodevelopmental milestones, and behavioral aspects). MRI-scans from the neonatal period, around 3 months and during childhood, if performed, were collected. The available amplitude-integrated EEGs (aEEGs) were analyzed for background pattern and epileptiform activity. Sleep-deprived (nap) EEGs and whole night recordings during follow-up were analyzed for background pattern, presence, location and quantity of epileptiform activity. Results of neurodevelopmental assessments, including screening of cognitive functioning, were collected. 


\section{Conventional MRI assessment}

MRIs from the neonatal period and around 3 months of age were re-assessed by an experienced neonatologist (LSdV) for the extent and location of thalamic injury. MRIs from childhood were assessed by an experienced pediatric neurologist (FEJ) for the same characteristics. Thalamic injury location was scored (anterior / ventral, medial or pulvinar).

\section{Thalamic volume and total brain volume}

Since MRI scans at 3 months are less influenced by acute injury (i.e. hemorrhage) than neonatal scans, these were used for thalamic and whole brain volumetry. Childhood MRI scans were made at different ages in our population and therefore considered less useful as an early prognostic marker. The software package Mevislab (https://www.mevislab. de/) was used to create manual segmentations of the thalamus using T1 / T2 images and perform semi-automatic volume-calculation. The thalamus was segmented per slice by one investigator (AFZ) with anatomical atlases as reference. The lateral / ventral border was defined using the posterior limb of the internal capsule as a landmark. The segmentations were checked by 3 investigators (NHCC, BvdM and FEJ) and adjusted where needed. Total brain volume was obtained using FSL (the FMRIB Software Library) for automatic segmentation of the T1 / T2 weighted images. The automatically segmented areas were visually inspected, the best segmentation was chosen and manually corrected if necessary. An example of a T1 weighted MRI of an included subject and segmentation of the thalamus and total brain volume is shown in figure 1.

\section{Diffusion tensor imaging (DTI)}

For patients whose MRI series (at 3 months and at childhood age) included DTI sequences, average bilateral thalamic fractional anisotropy (FA) and mean diffusivity (MD) values were determined. First, artefacts were corrected (e.g. signal drift, subject motion, eddy current induced geometric distortions) and scans were visually inspected for their quality. ${ }^{15-17}$ The scans of sufficient quality were further analyzed per T1-based region of interest (ROI), with the Oishi atlas as a reference template. ${ }^{18}$ DTI characteristics of the thalamus were calculated using ExploreDTI. ${ }^{19}$

\section{Neonatal electroencephalography (EEG)}

Two-channel aEEG with corresponding raw EEG data from the neonatal period were collected and analyzed. Background pattern and epileptiform activity during the earliest available 48 hours of aEEG were rated through pattern recognition by an experienced neonatologist. Background patterns were scored as continuous normal voltage (CNV, continuous trace, voltage 10-25 $(-50) \mu \mathrm{V}$ ), discontinuous normal voltage (DNV, discontinuous trace, voltage predominantly $>5 \mu \mathrm{V}$ ), burst-suppression (BS, discontinuous pattern; periods of very low voltage (inactivity) as well as higher amplitude bursts), 
continuous extremely low voltage (CLV, continuous pattern of very low voltage, around or $<5 \mu \mathrm{V}$ ), Flat tracing (FT: mainly inactive [isoelectric] trace, with very low voltage $<5 \mu \mathrm{V}$ ). Four categories of epileptiform activity were identified: No epileptiform activity (no EA), single seizure (SS: $<3$ discharges per 30 minutes), repetitive seizures (RS: $\geq 3$ discharges within 30 minutes), status epilepticus: "sawtooth" pattern.

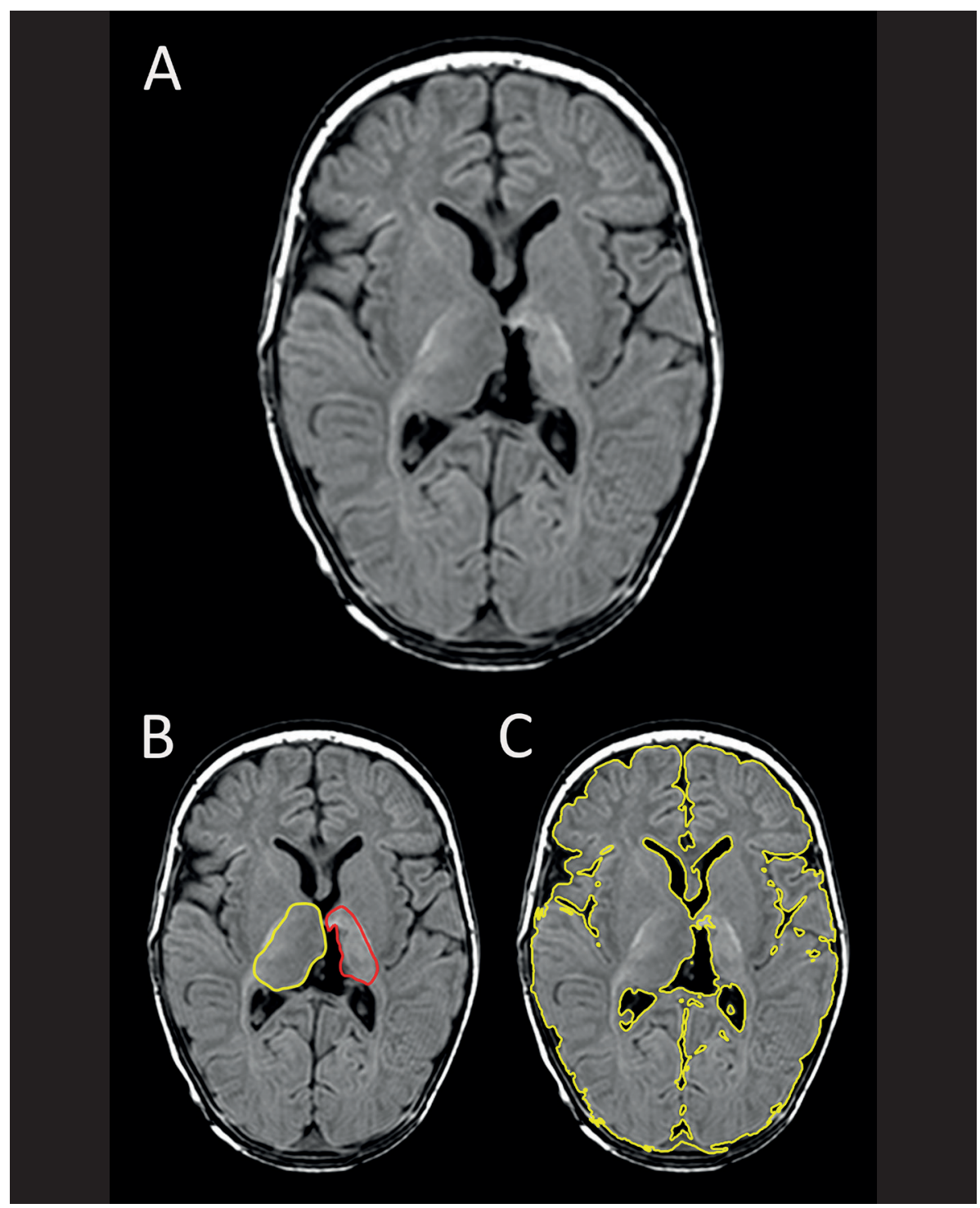

Figure 1. Illustrative MRI and segmentation in a 3 months-old patient with left-sided thalamic injury.

A. transversal slice of unprocessed T1 weighted MRI. B. manual segmentation of the thalamus. C. automatic segmentation the total brain volume. 


\section{EEG during follow-up}

Because of the known high risk of ESES development after perinatal thalamic injury, children with perinatal thalamic injury are routinely followed-up in our center with periodic sleepEEGs (either nap EEGs after sleep-deprivation or whole night registrations). All EEGs were evaluated for the presence of epileptiform activity (spike and wave complexes, spikes or sharp waves) in sleep. The follow-up EEG with the highest spike-wave index (SWI) was used as the "index EEG", because this was considered to best represent the severity of EEG abnormalities. The age at onset of ESES was variable in this population, thus an SWI assessment at the same age for all patients would not be representative. Also, the first (routine) EEG showing sleep activation of epileptiform activity may just be an early sign of the disease, while later EEGs show the full extent of abnormalities. The "index EEG" was assessed for background pattern, presence and distribution of epileptiform activity during wakefulness, sleep architecture and presence and quantification of epileptiform activity during sleep (SWI). The SWI was calculated in an epoch of 10 minutes (600 s) duration, starting 5 minutes after alpha attenuation or after sleep had clinically commenced. The number of seconds containing epileptiform discharges was divided by the total number of seconds in the epoch (600) and multiplied by 100 to reflect the SWI as a percentage. Classic ESES was defined as an SWI of above 85\% and ESES spectrum as an SWI of $50-85 \%$.

\section{Outcome definition}

Primary outcome for this study is the occurrence of epilepsy with ESES (-spectrum) abnormalities in the most severely affected EEG during follow-up. Secondary outcome is neurodevelopment as reflected by total Intelligence Quotient (preferred if available) or Developmental Quotient. Before the age of two years developmental quotients were obtained using the Griffiths mental development scales or the Bayley Scales of Infant and Toddler Development. After the age of two years the tests were chosen based on age and abilities of the children and included Wechsler Preschool and Primary Scale of Intelligence (WPPSI), Wechsler Intelligence Scale for Children (WISC III), Bayley Scales of Infant and Toddler Development, Griffiths mental development scales as well as the Snijders-Oomen non-verbal intelligence test (SON) in one case. We first performed our analyses with mean IQ / DQ after the age of two years as outcome measure. Subsequently, individual IQ / $\mathrm{DQ}$ measurements at all time points before and after the age of two years were used as outcome measure for a linear mixed model analysis.

\section{Statistical analysis}

An estimate of the risk of epilepsy with ESES after perinatal thalamic injury, while accounting for a difference in follow-up duration between patients, was provided by creating a KaplanMeier Curve for the prospectively followed cohort. Fisher's exact test was used to explore possible associations between thalamic and other injury lateralization and predominant 
EEG focus of epileptiform activity. The choice of parametric or non-parametric tests was based on the distribution of the data, using parametric tests (t-test / ANOVA) if the data did not clearly deviate from normal distribution, whereas for data that was not normally distributed, non-parametric (Mann-Whitney U test, Spearman's rank correlation) tests were used. The thalamic volume of patients who developed ESES(spectrum) was compared to that of patients who did not develop ESES (spectrum) with an independent samples t-test. Association between MRI-characteristics and SWI was assessed using Spearman's rank correlation coefficient for continuous and the Mann-Whitney $U$ test for categorical MRIvariables. A possible difference in mean IQ / DQ after two years of age according to the presence of injury in the different thalamic areas was assessed with independent samples t-tests. aEEG findings were tested for possible association to mean IQ / DQ after two years by means of a One-way Analysis Of Variance (ANOVA). Complete case univariable and multivariable linear regression analyses were performed with thalamic volume, total brain volume and SWI as possible predictors of mean total IQ / DQ after two years. 95\% confidence intervals ( $95 \% \mathrm{Cls}$ ) were calculated.

To account for differences in baseline intelligence levels between patients, and to include all IQ / DQ measurements despite difference in timing and tests between patients, a linear mixed model was fitted. All IQ / DQ measurements were defined as repeated outcome measures. The intercept was included as a random effect variable and thalamic volume, total brain volume and SWI were included as fixed variables. The covariance matrix model was chosen based on a comparison of the Akaike information criterion (AIC) of the model options in SPSS. The model with the Factor Analytic: First Order covariance matrix structure had the lowest AIC and was therefore considered to best fit the dataset.

P-values below 0.05 were considered significant. Statistical analysis was conducted using IBM SPSS Statistics Software version 25 by BvdM with advice and feedback by a clinical epidemiologist (JDJP).

\section{Standard Protocol Approvals, Registrations, and Patient Consents}

The study was approved by the medical ethics committee, who decided that the Dutch Medical Research Involving Human Subjects Act (WMO) did not apply.

\section{Data availability}

All anonymized data included in this study will be provided upon reasonable request to the corresponding author. 


\section{Results}

\section{Patient characteristics}

Twenty-three consecutive patients with perinatal thalamic injury were followed in the UMC Utrecht from the neonatal period onward and could be included in the prospectively followed (incidence) cohort. An additional seven patients, with perinatal thalamic injury, who were referred during childhood were included. Patient characteristics of the prospectively followed cohort and the whole cohort are shown in table 1.

Table 1. patient characteristics, clinical, EEG and neuropsychological findings

\begin{tabular}{|c|c|c|c|}
\hline \multicolumn{4}{|l|}{ neonatal period } \\
\hline & $\begin{array}{c}\text { All patients } \\
(n=30)\end{array}$ & $\begin{array}{c}\text { Prospectively } \\
\text { followed subgroup } \\
(n=23)\end{array}$ & $\begin{array}{l}\text { Retrospectively } \\
\text { added subgroup } \\
(n=7)\end{array}$ \\
\hline male $(\%)$ & $17 / 30(57 \%)$ & $15 / 23(65 \%)$ & $2 / 7(29 \%)$ \\
\hline gestational age: mean \pm sd & $39 \pm 2,5$ weeks & $39 \pm 2,5$ weeks & $38 \pm 2,7$ weeks \\
\hline pregnancy complications & $12 / 29(41 \%)$ & $11 / 23(48 \%)$ & $1 / 6(17 \%)$ \\
\hline delivery complications & $11 / 28(39 \%)$ & $11 / 23(48 \%)$ & $0 / 5(0 \%)$ \\
\hline birth weight: mean \pm sd & $3148( \pm 859) \mathrm{g}$ & $3151( \pm 877) \mathrm{g}$ & $3123( \pm 866) \mathrm{g}$ \\
\hline \multicolumn{4}{|l|}{ Apgar score at $1 / 5$ / 10 min: } \\
\hline mean & $7 / 9 / 9$ & $7 / 9 / 9$ & $7 / 9 /-$ \\
\hline median & $8 / 9 / 10$ & $8 / 9 / 10$ & $8 / 9 /-$ \\
\hline $\min$ & $4 / 5 / 6$ & $4 / 5 / 6$ & $4 / 8 /-$ \\
\hline $\max$ & $9 / 10 / 10$ & $9 / 10 / 10$ & $8 / 10 /-$ \\
\hline \multicolumn{4}{|l|}{ etiology: } \\
\hline sinus thrombosis & 20 & 17 & 3 \\
\hline arterial ischemic & 5 & 3 & 2 \\
\hline isolated hemorrhage & 2 & 2 & 0 \\
\hline infectious & 2 & 1 & 1 \\
\hline venous malformation & 1 & - & 1 \\
\hline neonatal convulsions & $24 / 30(80 \%)$ & $19 / 23(83 \%)$ & $5 / 7(71 \%)$ \\
\hline aEEG findings: & $n=15$ & $n=15$ & $\mathrm{~N} / \mathrm{A}$ \\
\hline PMA at aEEG: mean \pm sd & $40,6 \pm 1,4$ weeks & $40,6 \pm 1,4$ weeks & \\
\hline background*: CNV / DNV / BS / CLV / FT & $3 / 11 / 0 / 0 / 1$ & $3 / 11 / 0 / 0 / 1$ & \\
\hline epileptic activity & $13(87 \%)$ & $13(87 \%)$ & \\
\hline \multicolumn{4}{|l|}{ follow-up during childhood } \\
\hline duration of EEG follow-up: mean \pm sd & $95 \pm 40$ months & $96 \pm 40$ months & $93 \pm 42$ \\
\hline \multicolumn{4}{|l|}{ seizures: } \\
\hline focal & $12(40 \%)$ & $10(43 \%)$ & $2(29 \%)$ \\
\hline generalized & $4(13 \%)$ & $3(13 \%)$ & $1(14 \%)$ \\
\hline both & $5(17 \%)$ & $2(9 \%)$ & $3(43 \%)$ \\
\hline none & $9(30 \%)$ & $8(35 \%)$ & $1(14 \%)$ \\
\hline age at seizure onset: mean $\pm s d$ & $43 \pm 28$ months & $38 \pm 21$ months & $54 \pm 42$ months \\
\hline
\end{tabular}


Table 1. Continued

\begin{tabular}{|c|c|c|c|c|}
\hline \multicolumn{5}{|c|}{ EEG findings } \\
\hline wake: & normal background & $22(73 \%)$ & $20(87 \%)$ & $2(29 \%)$ \\
\hline \multirow[t]{4}{*}{ sleep: } & no SIEA & $3(10 \%)$ & $3(13 \%)$ & $0(0 \%)$ \\
\hline & SIEA & $1(3 \%)$ & $1(4 \%)$ & $0(0 \%)$ \\
\hline & ESES-spectrum & $8(27 \%)$ & $6(26 \%)$ & $2(29 \%)$ \\
\hline & classic ESES & $18(60 \%)$ & $13(57 \%)$ & $5(71 \%)$ \\
\hline \multicolumn{5}{|c|}{ Highest SWI in sleep: } \\
\hline \multirow{2}{*}{\multicolumn{2}{|c|}{$\begin{array}{l}\text { mean } \pm s d \\
\text { median }\end{array}$}} & $78 \pm 30 \%$ & $75 \pm 33 \%$ & $91 \pm 12 \%$ \\
\hline & & $91 \%$ & $89 \%$ & $98 \%$ \\
\hline \multicolumn{2}{|c|}{ range } & $0-100 \%$ & $0-100 \%$ & $70-100$ \\
\hline \multicolumn{2}{|c|}{ Number of anti-epileptic drugs used: mean \pm sd } & $3 \pm 3$ & $3 \pm 3$ & $4 \pm 3$ \\
\hline \multicolumn{5}{|c|}{$\begin{array}{l}\text { Neurodevelopment before documented ESES } \\
\text { (spectrum): }\end{array}$} \\
\hline \multicolumn{2}{|c|}{ normal } & $22(73 \%)$ & $18(78 \%)$ & $4(57 \%)$ \\
\hline \multicolumn{2}{|c|}{ intellectual impairment (IQ / DQ 70-85) } & $4(13 \%)$ & $3(13 \%)$ & $1(14 \%)$ \\
\hline \multicolumn{2}{|c|}{ intellectual disability $(I Q / D Q<70)$} & $4(13 \%)$ & $2(9 \%)$ & $2(29 \%)$ \\
\hline \multicolumn{5}{|c|}{$\begin{array}{l}\text { Dominant neurodevelopmental abnormalities during } \\
\text { follow-up / after ESES onset: }\end{array}$} \\
\hline \multicolumn{2}{|c|}{ global cognitive impairment } & $13(43 \%)$ & $9(39 \%)$ & $4(57 \%)$ \\
\hline \multicolumn{2}{|c|}{ language impairment } & $5(17 \%)$ & $3(13 \%)$ & $2(29 \%)$ \\
\hline \multicolumn{2}{|c|}{ behavioral disorder } & $1(3 \%)$ & $1(4 \%)$ & $0(0 \%)$ \\
\hline \multicolumn{2}{|c|}{ none } & $11(37 \%)$ & $10(44 \%)$ & $1(14 \%)$ \\
\hline \multicolumn{5}{|c|}{ Severity of ESES related impairment: } \\
\hline \multicolumn{2}{|c|}{ regression } & $5(17 \%)$ & $2(9 \%)$ & $3(43 \%)$ \\
\hline \multicolumn{2}{|c|}{ arrest } & $9(30 \%)$ & $6(26 \%)$ & $3(43 \%)$ \\
\hline \multicolumn{2}{|c|}{ delay } & $5(17 \%)$ & $5(22 \%)$ & $0(0 \%)$ \\
\hline \multicolumn{2}{|c|}{ none } & $11(37 \%)$ & $10(44 \%)$ & $1(14 \%)$ \\
\hline \multicolumn{5}{|c|}{ Total IQ / DQ during follow-up (mean \pm sd) at } \\
\hline \multicolumn{2}{|c|}{$\sim 6$ months } & $102 \pm 14, n=15$ & $102 \pm 14, n=15$ & $N / A$ \\
\hline & $\sim 12$ months & $93 \pm 20, n=23$ & $94 \pm 18, n=22$ & $55, n=1$ \\
\hline & $\sim 24$ months & $90 \pm 15, n=22$ & $92 \pm 13, n=21$ & $55, n=1$ \\
\hline & $\sim 2-5$ years & $86 \pm 19, n=16$ & $89 \pm 18, n=15$ & $55, n=1$ \\
\hline & $\sim 5-8$ years & $82 \pm 17, n=16$ & $89 \pm 17, n=12$ & $71, n=4$ \\
\hline & $\sim 8-13$ years & $66 \pm 16, n=10$ & $73 \pm 17, n=6$ & $56, n=4$ \\
\hline
\end{tabular}

\section{Table legend:}

sd: standard deviation, aEEG: amplitude-integrated EEG, PMA: post-menstrual age, CNV: continuous normal voltage,

DNV: discontinuous normal voltage, BS: burst-suppression, CLV: continuous extremely low voltage, FT: flat trace, N/A: Not available, SWI = spike-wave index, SIEA = sleep induced epileptiform activity

* Background is defined as worst background pattern and might have been influenced by medication. The patient with a FT background pattern was administered clonazepam and phenobarbital during aEEG registration. After six hours of severe aEEG depression his background showed marked improvement.

\section{Neurodevelopmental findings during follow-up}

A gradual decrease in mean IQ / DQ was seen with age (table 1). When comparing IQ / DQ before ESES was first detected (at 24 months) with the first IQ / DQ after ESES (spectrum) diagnosis for patients for whom these data were available at both timepoints $(n=18)$ a decrease from a mean IQ of 89 to 82 ( $p=0.046$, average time interval 43 months) was found. 


\section{MRI findings (table 2)}

In the total cohort of 30 patients, MR images of sufficient quality for clinical assessment were available from the neonatal period in 24 children, around three months of age in 23, and during childhood in 22 patients. The medial part of the thalamus was most often involved in the injury at the neonatal and three months MRIs, while at the childhood MRI the sequelae of the perinatal thalamic injury were more diffuse (table 2). Mean total (bilateral) thalamic volume at three months was $8.11 \mathrm{ml}$. A small mean increase in FA was seen from age three months into childhood (from 0.240 to 0.278 ).

\section{Primary findings}

\section{Occurrence of ESES (spectrum) abnormalities during follow up}

Mean EEG follow-up was 96 months in the prospectively followed cohort. Most patients (87\%) had a normal background pattern during wakefulness, while in sleep 6 patients had ESES-spectrum abnormalities (26\%) and an additional 13 patients showed a classic ESES pattern ( $57 \%$, table 1). The ESES(-spectrum) abnormalities were limited to one hemisphere in 8 cases (42\%) and bilateral in 11 cases (58\%). There was no significant difference in EEG follow-up between the patients with ESES spectrum abnormalities and those with classic ESES (93 vs 103 months, $p=0.62$ ). Figure 2 shows a Kaplan Meier curve for the occurrence of ESES (spectrum) in the prospectively followed cohort.

\section{Imaging predictors for the occurrence of ESES (-spectrum) and for the severity of neurodevelopmental abnormalities}

A large majority (19 of 23) of the prospectively followed patients developed ESES (-spectrum) abnormalities during follow-up. The patients who developed ESES, had a lower thalamic volume (mean \pm sd $7.8 \pm 1.5 \mathrm{ml}$ vs. $9.7 \pm 1.5 \mathrm{ml}, \mathrm{p}=0.04$ ). Because of the very small group of patients $(n=4)$ who did not develop ESES (spectrum) abnormalities, we considered statistical power too small to compare other characteristics between the two groups.

Larger thalamic volume and larger total brain volume, measured on three months MRI, were correlated to higher mean IQ / DQ after the age of 2 years ( $r=0.74, p=0.001$ and $r=0.64, p=0.005$, table 3 and supplementary figure 1). Injury involving the anterior / ventral portion of the thalamus was the only of the three predefined areas that was associated with mean IQ / DQ after 2 years of age (mean IQ / DQ 72 for those with vs. 89 for those without anterior / ventral injury, $\mathrm{p}=0.04, \mathrm{n}=19$ ). In a subgroup of patients with unilateral thalamic injury, according to visual inspection of the 3 months MRI $(n=18)$, the volume of the affected thalamic hemisphere was not correlated with, while the volume of the contralateral thalamic hemisphere was positively correlated with mean IQ / DQ after the age of 2 years $(r=0.38, p=0.17$ and $r=0.84, p<0.001$, table 3$)$. 
Chapter 2. Outcome after perinatal thalamic injury

Table 2. MRI findings

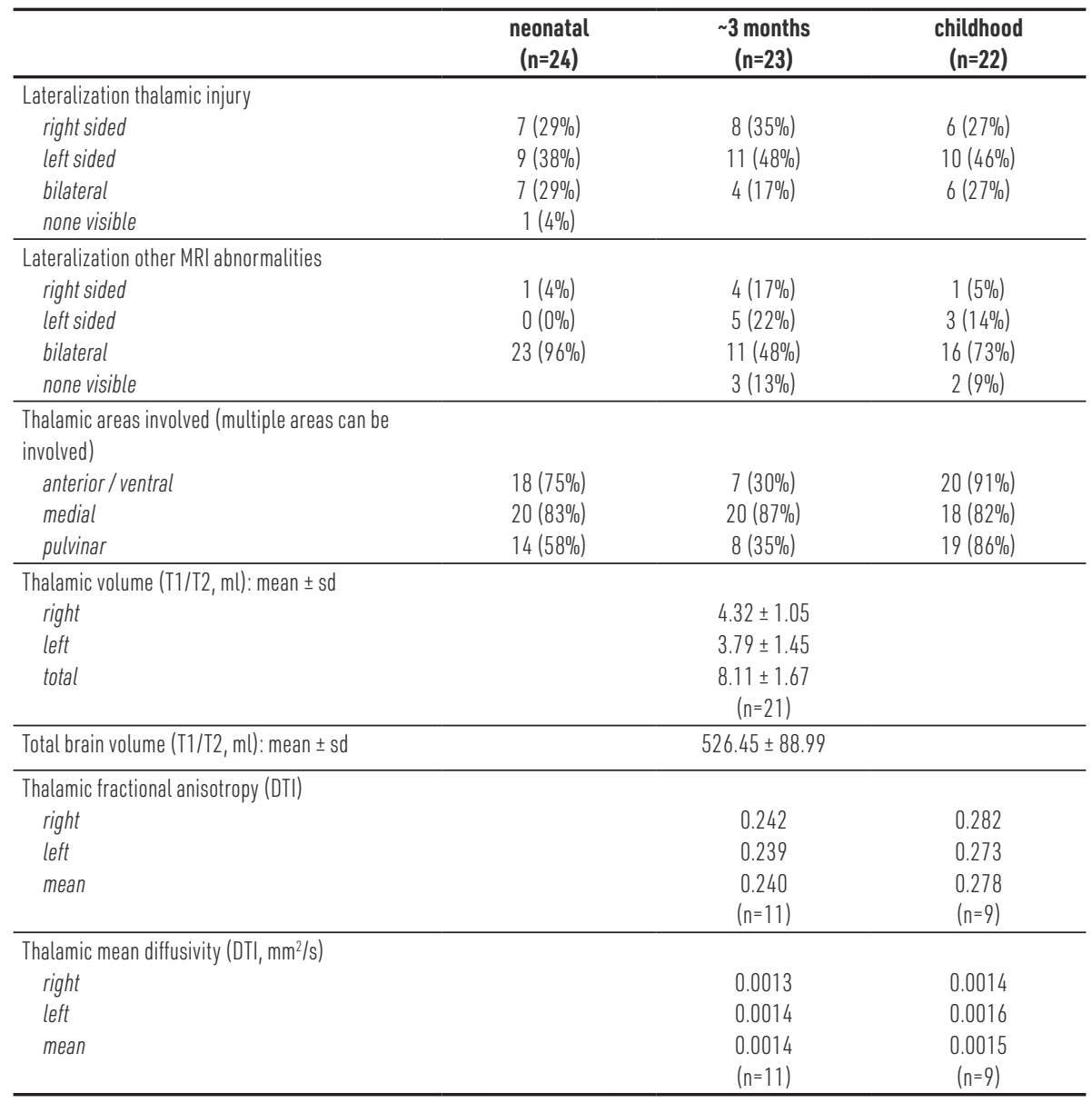

Multivariable linear regression analysis revealed that mean IQ / DQ after the age of two years was correlated with thalamic volume ( $\beta 6.4$ [95\% Cl 0.65 - 12.1]), but no longer with total brain volume $(\beta 0.05[95 \% \mathrm{Cl}-0.06-0.16])$ and SWI $(\beta 0.08$ [95\% Cl -0.12 - 0.27]). Thus, corrected for total brain volume and SWI, a $1 \mathrm{ml}$ higher thalamic volume was associated with an increase of 6.4 IQ points in this analysis.

With a linear mixed model we found that thalamic volume was an independent predictor of IQ / DQ (univariable coefficient 7.2 [95\% Cl 5.3 - 9.1], multivariable coefficient 9.6 [95\% CI 5.9 - 13.3]), while total brain volume and SWI were not significantly correlated with IQ / DQ (table 4). This means that, corrected for total brain volume and SWI and accounting for repeated measures within patients, a $1 \mathrm{ml}$ higher thalamic volume was associated with a 9.6 points higher $1 Q$ 


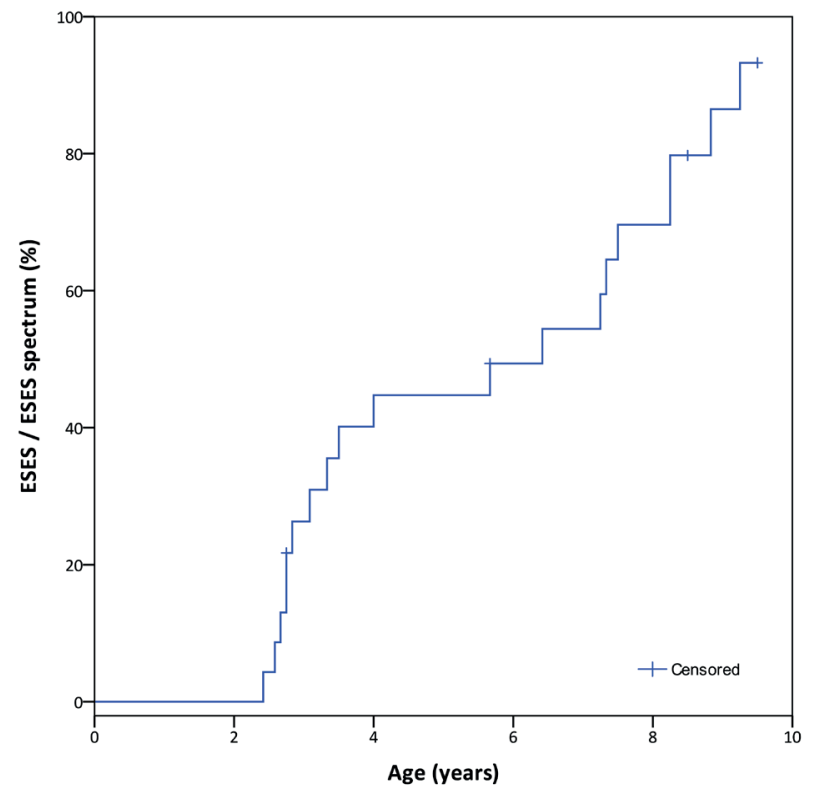

Figure 2. Kaplan-Meier curve for ESES or ESES spectrum in incidence cohort $(n=23)$

The percentage of patients with perinatal thalamic injury with ESES / ESES spectrum related to age.

Exploratory analysis of DTI characteristics and neurodevelopment during follow-up

Thalamic DTI characteristics at three months were not predictive for mean IQ / DQ after the age of 2 years. Childhood MRI DTI characteristics, however, were correlated with mean IQ / DQ after the age of two years: higher FA and lower MD predicted higher IQ ( $r=0.76$, $p=0.03$ and $r=-0.81, p=0.02$ respectively, table 3 ).

\section{Additional findings}

Correlation of MRI characteristics to epileptiform activity during follow-up

9 of 11 patients with unilateral thalamic injury and a lateralized EEG focus in sleep had their predominant EEG focus on the same side as their thalamic injury focus. Nonetheless, lateralization of thalamic injury ( $p=0.09)$, or lateralization of other MRI abnormalities ( $p$ $=0.99$ ) were not correlated to the predominant localization of epileptiform activity in sleep. There was no association between the affected thalamic areas at the three months MRI and SWI during follow-up. Thalamic volume at the three months MRI showed a trend towards correlation, while total brain volume did not show a correlation with SWI during follow-up (rho -0.42; $p=0.06$ and rho $-0.23 ; p=0.32, n=21$ ). DTI characteristics at three months ( $n=11$ ) were not correlated to SWI either (rho $0.58 ; p=0.06$ for FA and rho $-0.07 ; p=0.82$ for MD), while DTI characteristics at the childhood MRI ( $n=9)$ were strongly correlated to SWI: a higher thalamic FA was correlated to lower SWI (rho - $0.81, p=0.008$, table 3) and higher thalamic MD was correlated to higher SWI (rho 0.72, $p=0.03$, table 3). 
Table 3. Correlation of MRI characteristics and SWI / IQ during follow-up

\begin{tabular}{|c|c|c|c|c|}
\hline & \multicolumn{2}{|c|}{ SWI (\%) } & \multicolumn{2}{|c|}{ IQ } \\
\hline & rho & p & $\mathbf{r}$ & p \\
\hline \multicolumn{5}{|l|}{ volumetric measurements at 3 months MRI $(n=21)$} \\
\hline total thalamic volume (ml) & -0.42 & 0.06 & 0.74 & 0.001 \\
\hline unilateral cases*: volume of affected side (ml) & -0.39 & 0.11 & 0.38 & 0.17 \\
\hline unilateral cases*: volume of contralateral side (ml) & -0.34 & 0.16 & 0.84 & $<0.001$ \\
\hline total brain volume (ml) & -0.23 & 0.32 & 0.64 & 0.005 \\
\hline \multicolumn{5}{|l|}{ DTI characteristics } \\
\hline \multicolumn{5}{|l|}{ 3 months DTI $(n=11)$} \\
\hline thalamic fractional anisotropy (FA) & 0.58 & 0.06 & 0.03 & 0.93 \\
\hline thalamic mean diffusivity (MD) & -0.07 & 0.82 & 0.42 & 0.26 \\
\hline \multicolumn{5}{|l|}{ childhood DTI (n=9) } \\
\hline thalamic fractional anisotropy (FA) & -0.81 & 0.008 & 0.76 & 0.03 \\
\hline thalamic mean diffusivity (MD) & 0.72 & 0.03 & -0.81 & 0.02 \\
\hline
\end{tabular}

\section{Table legend:}

rho $=$ Spearman's rho, $r=$ Pearson's correlation coefficient

${ }^{*} \mathrm{n}=18$ cases with unilateral thalamic injury based visual inspection of the $\sim 3$ months MRI

Correlation of aEEG, and SWI to neurodevelopmental findings during follow-up The aEEG background pattern and presence / severity of epileptiform activity in the neonatal period were not correlated to mean IQ / DQ after two years of age ( $p=0.43$ and $p=0.62, n=14$ with available aEEG from the neonatal period and neurodevelopmental follow-up after the age of 2 years). Higher SWI was correlated to lower IQ during follow-up (rho $-0.44, p=0.03$ ).

\section{Multivariable analysis and linear mixed model}

Table 4. Possible predictors of cognitive function during follow-up

\begin{tabular}{|c|c|c|}
\hline \multicolumn{3}{|c|}{ Linear regression analysis of mean IQ outcome, at age $>2$ years } \\
\hline & $\begin{array}{l}\text { Univariable } \\
\beta(95 \% \mathrm{Cl}) \\
\end{array}$ & $\begin{array}{l}\text { Multivariable } \\
\beta(95 \% \mathrm{CI})\end{array}$ \\
\hline thalamic volume (ml) & $7.3(3.7-11.0)$ & $6.4(0.6-12.1)$ \\
\hline total brain volume (ml) & $0.13(0.04-0.21)$ & $0.05(-0.06-0.16)$ \\
\hline SWI $(\%)$ & $-0.19(-0.42-0.04)$ & $0.08(-0.12-0.27)$ \\
\hline \multicolumn{3}{|c|}{ Mixed linear model with DQ / IQ measurements as repeated measures } \\
\hline & $\begin{array}{l}\text { Univariable coefficient } \\
(95 \% \mathrm{CI})\end{array}$ & $\begin{array}{l}\text { Multivariable coefficient } \\
(95 \% \mathrm{CI})\end{array}$ \\
\hline thalamic volume (ml) & $7.2(5.3-9.1)$ & $9.6(5.9-13.3)$ \\
\hline total brain volume (ml) & $0.07(-0.02-0.15)$ & $-0.06(-0.13-0.01)$ \\
\hline SWI (\%) & $-0.18(-0.38-0.02)$ & $0.02(-0.12-0.16)$ \\
\hline
\end{tabular}

\section{Table legend:}

DQ: developmental quotient, IQ: Intelligence Quotient, SWI: Spike-wave index 


\section{Discussion}

In this study, we show that 1) the majority of patients with perinatal thalamic injury eventually develop epilepsy with classic ESES (SWI>85\%) or ESES spectrum (SWI 50-85\%),

2) Patients with perinatal thalamic volume who develop ESES(-spectrum) during follow-up had a lower thalamic volume at 3 months as compared to patients who did not develop ESES(-spectrum). Furthermore, higher residual thalamic volume at three months of age correlates with higher IQ / DQ at follow-up - also after correction for total brain volume and spike wave index - and 3) thalamic DTI indices during childhood correlate with the severity of epileptiform activity in sleep and with cognitive functioning.

The Kaplan Meier curve of our incidence cohort suggests that more than $90 \%$ of children with perinatal thalamic injury develop epilepsy with ESES spectrum abnormalities during follow-up. Previous studies also suggested a strong link between thalamic injury and ESES spectrum abnormalities. ${ }^{1,2}$ The current study is the first to confirm this in a prospectively followed cohort with long-term follow-up. As almost all patients had ESES during follow-up, we were unable to find predictors of the occurrence of ESES within the population of children with thalamic injury. Several possible mechanisms behind the occurrence of ESES in children with thalamic abnormalities have been proposed. The thalamus has the capacity to generate rhythmic oscillations (e.g. sleep spindles) in a thalamocortical circuit that is activated during sleep. These oscillations are thought to result from balanced activity between thalamic excitatory neurons and $\gamma$-aminobutyric acid (GABA)ergic inhibitory neurons. ${ }^{20}$ Loss or dysfunction of thalamic inhibitory neurons may lead to a pathologic derangement of these oscillations during sleep, as reflected by rhythmic spike and wave discharges., 71,22 As an additional mechanism, a recent study suggested that loss of thalamic control of plasticity of part of the cortex (i.e. incomplete loss of thalamocortical connectivity) leads to a pathological "augmentation response" and thereby a hyperexcitable cortical network. ${ }^{9}$

We investigated whether neurodevelopment can be predicted by the residual thalamic volume and found a strongly significant correlation: higher total thalamic volume at three months of age predicted higher IQ / DQ during follow-up. In cases with unilateral thalamic injury based on inspection of the three months MRI by the clinician, the volume of the contralateral thalamus was significantly correlated to mean IQ / DQ during follow-up while the ipsilateral thalamus was not. This suggests that the cognitive performance depends mainly on the least affected side. It could be that, although normal on visual inspection, more subtle changes in structure and function of the contralateral thalamic hemisphere are also present and that volume of the contralateral thalamic hemisphere is indicative of the compensatory capabilities. 
Chapter 2. Outcome after perinatal thalamic injury

Previous studies in both adults and children also found a correlation of thalamic volume and thalamocortical connections with aspects of cognitive functioning. ${ }^{23,24}$ Recent studies have suggested that the thalamus has an important role in sustaining attention, not only by filtering information to and between cortical regions, but also by enhancing the functional connectivity of relevant cortical areas. ${ }^{25}$ We hypothesized that, in addition to volume loss, altered structural organization of the thalamus could explain the cognitive deficits that are seen in children with epilepsy with ESES. By analyzing DTI characteristics of the thalamus, we explored this hypothesis. Although we did not find such a correlation when DTI measures were studied at the age of three months, higher thalamic FA and lower MD on DTI scans acquired during childhood were associated with higher IQ / DQ, suggesting that thalamic microstructure, as well as its volume, is important for neurodevelopment. Also, we found that higher FA and lower MD on later DTI scans were associated with lower SWI, which may reflect that more organized thalamic microstructure is associated with less epileptiform activity in sleep.

We found that a higher percentage of epileptiform activity, expressed as the spike-wave index, correlates to lower IQ after perinatal thalamic injury. ESES may be a factor that influences cognitive functioning in addition to the impact of the thalamic injury itself, or it could merely be a marker of severity of thalamic injury. Previous studies suggested that ESES itself contributes to cognitive deficits ${ }^{26-28}$ and that improvement of cognitive functioning can be expected if epileptiform activity is reduced with treatment. ${ }^{29}$ However, in these papers, wider populations were studied, including children in whom an underlying etiology was not detected. Future studies comparing patients with ESES after thalamic injury and patients with ESES of non-structural etiology (although this also is a heterogeneous group) may further elucidate whether the thalamic injury or the ESES is the main determinant of long-term neurodevelopment. In our multivariable analysis we did not find a significant correlation of SWI with IQ. A possible explanation is that, for each patient, we included the EEG with the highest SWI and did not consider that in some cases the epileptiform activity was reduced or disappeared with treatment.

Our study is the first to quantitatively assess the risk of epilepsy with ESES after thalamic injury in a prospectively followed cohort and combine multiple modalities (MR volumetry, DTI and EEG) as well as repeated clinical / neuropsychological assessments to reveal predictors of cognitive functioning.

Our results have to be interpreted with care. First, the relatively small and somewhat heterogeneous study population (inherent to the rarity of perinatal thalamic injury) inevitably results in some uncertainty regarding the cumulative incidence of epilepsy with ESES after thalamic injury. Second, although MRI acquisition in our center was done 
according to a standard schedule, MRI protocols changed during the period of conduct of our study, which results in variability of available imaging data. Especially our analysis of DTI characteristics should be seen as explorative as these sequences were available in a minority of patients. Our DTI data were of insufficient quality and quantity for reliable analysis of thalamocortical tracts and this would be an interesting subject to address in a future study. Although developmental screening in the first two years was done according to a standard protocol, follow-up afterwards with full neuropsychological assessment was performed at variable time points and may have been selective (patients with learning problems could have been tested more often). Lastly, the use of a multivariable mixed model analysis allowed the use of multiple TIQ measurements per patient and adjusts for the fact that each patient has his / her own "baseline" total IQ. However, using this multivariable mixed model in our relatively small study population, may have led to overfitting and could limit the generalizability of our results.

The results of our study implicate that clinicians treating children with perinatal thalamic injury should be aware of the risk of epilepsy with ESES. We recommend careful followup with assessment of cognitive functioning and serial sleep EEGs after the age of two years in these children. Parents should be made aware that a cognitive or behavioral deterioration of their child needs prompt evaluation. In case of ESES accompanied by a cognitive decline, treatment should be initiated soon, because early treatment of epilepsy with ESES can improve neurodevelopmental outcome. 14,27,29,30

In conclusion, perinatal thalamic injury is often followed by electrical status epilepticus in sleep in children after the age of two years. Cognitive performance of these children has a strong correlation with residual thalamic volume. The cognitive deficits in these patients may also be explained by abnormal structural organization of the thalamus as reflected by DTI characteristics. Future studies may elucidate whether loss of physiological thalamocortical connections explains these cognitive deficits.

\section{Acknowledgements}

This study was funded by the Dutch Epilepsy Fund (Epilepsiefonds, grant 13-17) and the Wilhelmina Children's Hospital Research Fund (grant R2634). The funding sources had no direct involvement in the conduct of the study.

We thank Mona C Toet for her help with the analysis of the neonatal amplitude-integrated EEGs, Floris Groenendaal for his help with the MRI assessment and Sophie Y van Driel for her help with the design of the included figures. 
Chapter 2. Outcome after perinatal thalamic injury

\section{Disclosures}

None of the authors has any conflict of interest to disclose.

\section{Ethical Publication Statement}

We confirm that we have read the Journal's position on issues involved in ethical publication and affirm that this report is consistent with those guidelines. 


\section{References}

1. Guzzetta F, Battaglia D, Veredice C, Donvito V, Pane M, Lettori D, et al. Early thalamic injury associated with epilepsy and continuous spike-wave during slow sleep. Epilepsia. 2005; 46(6):889-900.

2. Kersbergen KJ, De Vries LS, Leijten FSS, Braun KPJ, Nievelstein R a J, Groenendaal F, et al. Neonatal thalamic hemorrhage is strongly associated with electrical status epilepticus in slow wave sleep. Epilepsia. 2013; 54(4):733-40.

3. Zubiaurre-Elorza L, Soria-Pastor S, Junqué C, Fernandez-Espejo D, Segarra D, Bargalló N, et al. Thalamic changes in a preterm sample with periventricular leukomalacia: Correlation with whitematter integrity and cognitive outcome at school age. Pediatr Res. 2012; 71(4):354-60.

4. Kersbergen KJ, Groenendaal F, Benders MJNL, Van Straaten HLM, Niwa T, Nievelstein RAJ, et al. The spectrum of associated brain lesions in cerebral sinovenous thrombosis: Relation to gestational age and outcome. Arch Dis Child Fetal Neonatal Ed. 2011; 96(6).

5. Merlini L, Hanquinet S, Fluss J. Thalamic Hemorrhagic Stroke in the Term Newborn: A Specific Neonatal Syndrome with Non-uniform Outcome. J Child Neurol. 2017; 32(8):746-53.

6. Fernández IS, Takeoka M, Tas E, Peters JM, Prabhu SP, Stannard KM, et al. Early thalamic lesions in patients with sleep-potentiated epileptiform activity. Neurology. 2012; 78(22):1721-7.

7. Siniatchkin M, Groening K, Moehring J, Moeller F, Boor R, Brodbeck V, et al. Neuronal networks in children with continuous spikes and waves during slow sleep. Brain. 2010; 133(9):2798-813.

8. Bartolini E, Falchi M, Zellini F, Parrini E, Grisotto L, Cosottini M, et al. The syndrome of polymicrogyria, thalamic hypoplasia, and epilepsy with CSWS. Neurology. 2016; 86(13):1250-9.

9. Leal A, Calado E, Vieira JP, Mendonça C, Ferreira JC, Ferreira H, et al. Anatomical and physiological basis of continuous spike-wave of sleep syndrome after early thalamic lesions. Epilepsy Behav. 2018; 78:243-55.

10. Patry G, Lyagoubi S, Tassinari CA. Subclinical "electrical status epilepticus" induced by sleep in children. Arch Neurol. 1971; 24(3):242-52.

11. Landau WM, Kleffner FR. Syndrome of acquired aphasia with convulsive disorder in children. Neurology. 1957; 7(8):1241-9.

12. Scheltens-De Boer M. Guidelines for EEG in encephalopathy related to ESES/CSWS in children. In: Epilepsia. 2009. p. 13-7.

13. Nickels K, Wirrell E. Electrical Status Epilepticus in Sleep. Semin Pediatr Neurol. 2008; 15(2):50-60.

14. Van Den Munckhof B, Van Dee V, Sagi L, Caraballo RH, Veggiotti P, Liukkonen E, et al. Treatment of electrical status epilepticus in sleep: A pooled analysis of 575 cases. Epilepsia. 2015; 56(11):1738-46.

15. Vos SB, Tax CMW, Luijten PR, Ourselin S, Leemans A, Froeling M. The importance of correcting for signal drift in diffusion MRI. Magn Reson Med. 2017; 77(1):285-99.

16. Leemans A, Jones DK. The B-matrix must be rotated when correcting for subject motion in DTI data. Magn Reson Med. 2009; 61(6):1336-49.

17. Jones DK, Leemans A. Diffusion tensor imaging. Methods Mol Biol. 2011; 711:127-44.

18. Oishi K, Mori S, Donohue PK, Ernst T, Anderson L, Buchthal S, et al. Multi-contrast human neonatal brain atlas: Application to normal neonate development analysis. Neuroimage. 2011; 56(1):8-20.

19. Leemans A, Jeurissen B, Sijbers J, Jones DK. ExploreDTI: a graphical toolbox for processing, analyzing, and visualizing diffusion MR data. In: Proceedings 17th Scientific Meeting International Society for Magnetic Resonance in Medicine. 2009. p. 3537.

20. Steriade M. Sleep, epilepsy and thalamic reticular inhibitory neurons. Vol. 28, Trends in Neurosciences. 2005. p. 317-24.

21. Beenhakker MP, Huguenard JR. Neurons that Fire Together Also Conspire Together: Is Normal Sleep Circuitry Hijacked to Generate Epilepsy? Vol. 62. 2009. p. 612-32. 
Chapter 2. Outcome after perinatal thalamic injury

22. Agarwal R, Kumar A, Tiwari VN, Chugani H. Thalamic abnormalities in children with continuous spike-wave during slow-wave sleep: An F-18-fluorodeoxyglucose positron emission tomography perspective. Epilepsia. 2016; 57(2):263-71.

23. Hughes EJ, Bond J, Svrckova P, Makropoulos A, Ball G, Sharp DJ, et al. Regional changes in thalamic shape and volume with increasing age. Neuroimage. 2012;

24. Ball G, Pazderova L, Chew A, Tusor N, Merchant N, Arichi T, et al. Thalamocortical connectivity predicts cognition in children born preterm. Cereb Cortex. 2015; .

25. Halassa MM, Kastner S. Thalamic functions in distributed cognitive control. Nat Neurosci. 2017;

26. Scholtes FBJ, Hendriks MPH, Renier WO. Cognitive deterioration and electrical status epilepticus during slow sleep. Epilepsy Behav. 2005; 6(2):167-73.

27. Pera MC, Brazzo D, Altieri N, Balottin U, Veggiotti P. Long-term evolution of neuropsychological competences in encephalopathy with status epilepticus during sleep: A variable prognosis. Epilepsia. 2013; 54(SUPPL.7):77-85.

28. Tassinari CA, Rubboli G. Cognition and paroxysmal EEG activities: From a single spike to electrical status epilepticus during sleep. Epilepsia. 2006; 47(SUPPL. 2):40-3.

29. van den Munckhof B, Alderweireld C, Davelaar S, van Teeseling HC, Nikolakopoulos S, Braun KPJ, et al. Treatment of electrical status epilepticus in sleep: Clinical and EEG characteristics and response to 147 treatments in 47 patients. Eur J Paediatr Neurol. 2018; 22(1):64-71.

30. Maltoni L, Posar A, Parmeggiani A. Long-term follow-up of cognitive functions in patients with continuous spike-waves during sleep (CSWS). Epilepsy Behav. 2016; 60:211-7. 


\section{Supplementary material}

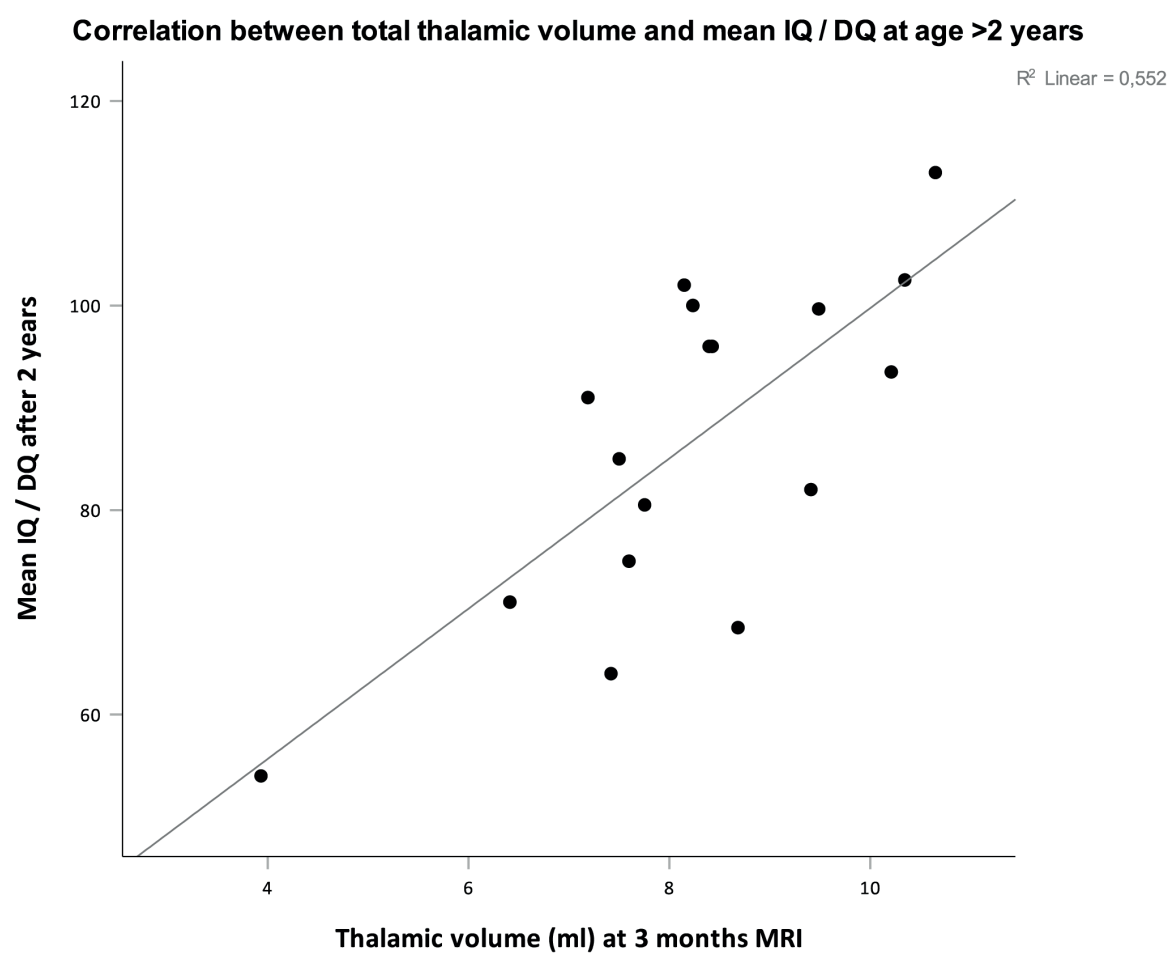

Figure S1. Correlation between total thalamic volume and mean IQ / DQ at age $>2$ years

The mean intelligence quotient / developmental quotient related to thalamic volume (ml) at the MRI at three months of age. 

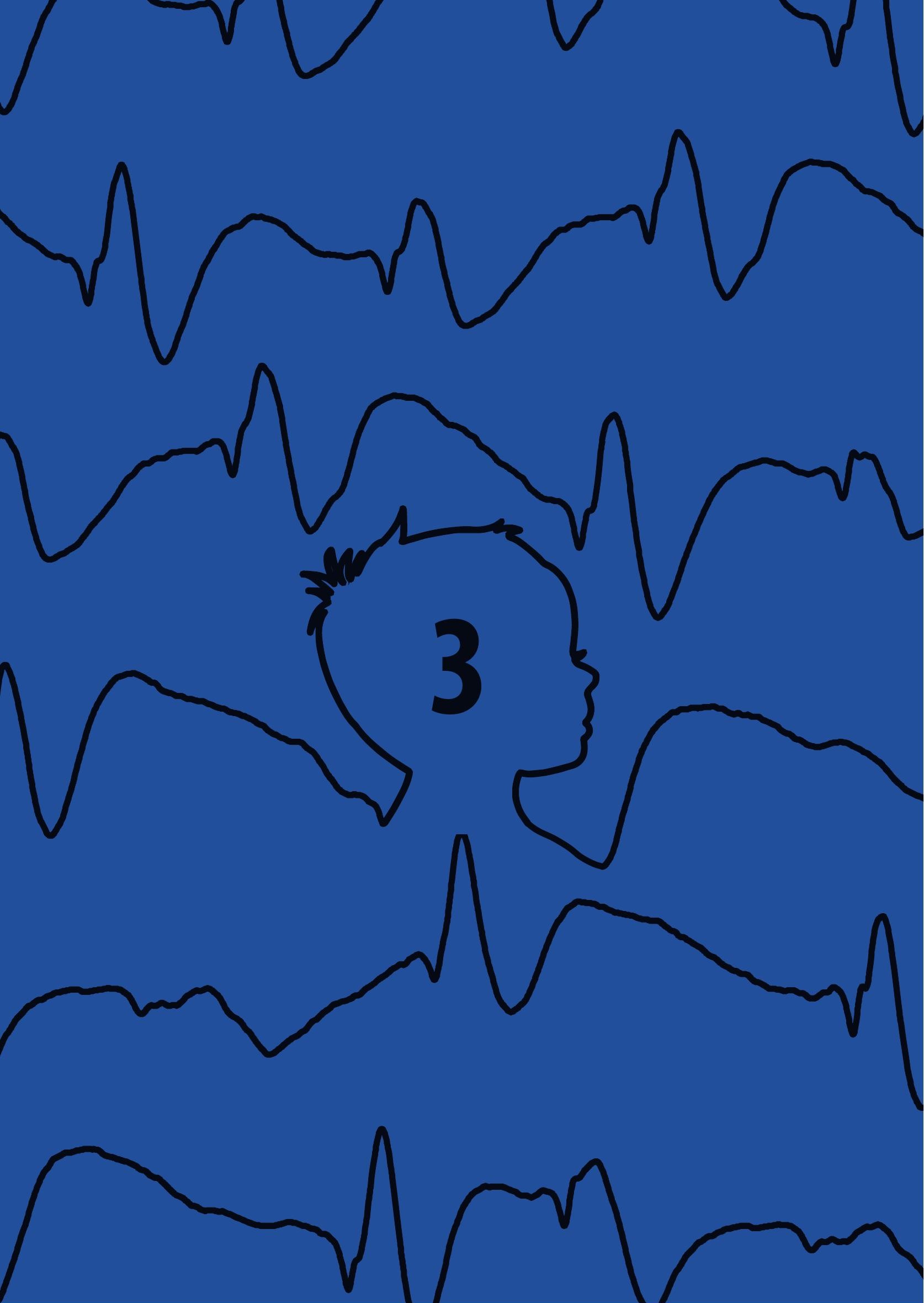
Chapter 3

\section{Inflammatory mediators in human epilepsy: a systematic review and meta-analysis}

Evelien E de Vries ${ }^{1}$

Bart van den Munckhof'

Kees PJ Braun

Annet van Royen-Kerkhof

Wilco de Jager ${ }^{2}$

Floor E Jansen ${ }^{2}$

${ }^{1}$ These first authors contributed equally

${ }^{2}$ These last authors contributed equally

Neuroscience \& Biobehavioral Reviews. 2016; 63:177-190 


\section{Abstract}

Background: Accumulating evidence suggests a role for inflammation in the pathophysiology of epilepsy.

Methods: We performed a systematic review and meta-analysis of studies that investigated inflammatory mediators in human epilepsy. Studies reporting on inflammatory mediators in serum, cerebrospinal fluid or brain tissue of epilepsy patients were included. Studies comparing patients to controls were included in a meta-analysis.

Results: 66 articles reporting on 1934 patients were included. IL-1 ra, IL-1 $\beta$, IL-6, IL-10, IFN- $\gamma$ and TNF-a were the most extensively investigated proteins. Elevated levels for IL-1 ra, IL1 $\beta$, IL-6 and CXCL8/IL-8 were reported in several different epilepsy etiologies and media, while other proteins were specifically increased for one etiology. IL-1a, IL-7 and IL-13, as well as the chemokines CCL2-5,-19 and -22, were increased exclusively in brain tissue. In an aggregate meta-analysis, we found significantly different protein levels for serum IL-6, IL-17 and CSF IL-1 $\beta$ and IL-10.

Conclusion: Inflammatory pathways are involved in epilepsy. Future studies may further clarify their role, and prove potential of targeted anti-inflammatory treatment.

\section{Highlights}

- A total of 66 articles on inflammation and epilepsy were systematically analyzed.

- A meta-analysis revealed elevated levels of serum IL-6, IL-17 and CSF IL-1 $\beta, I L-10$.

- There is substantial overlap between findings in serum, CSF and brain tissue.

- Many different chemokines were found elevated in brain tissue.

- Future studies should explore the use of targeted anti-cytokine therapies. 


\section{Introduction}

Epilepsy is one of the most common, often chronic, neurological disorders that affects up to $1 \%$ of the people worldwide and is characterized by an enduring predisposition of the brain to generate epileptic seizures ' . It is considered a heterogeneous group, with diverse etiology (genetic, structural, metabolic, unknown) and includes specific, often age-related, electroclinical syndromes. Seizures can be classified as either generalized (e.g. typical absences or myoclonic seizures) or focal. Temporal lobe epilepsy is the most prevalent form of focal epilepsy and is often associated with hippocampal sclerosis. Epileptic encephalopathies are increasingly recognized as conditions in which the epileptic activity itself may contribute to severe cognitive and behavioral abnormalities, beyond what can be expected from the underlying pathology alone. Examples include West syndrome and Dravet syndrome, presenting in infancy, and Lennox-Gastaut syndrome and epileptic encephalopathy with electrical status epilepticus in sleep (ESES) presenting during childhood ${ }^{2,3}$. Despite adequate treatment with antiepileptic drugs, about $30 \%$ of patients with epilepsy will continue to experience seizures and are therefore considered to have pharmacoresistant epilepsy ${ }^{4,5}$. Today's treatments mostly aim to suppress seizures, while the underlying pathophysiological mechanisms are not targeted as these are incompletely understood. Understanding the process of epileptogenesis may lead to pathophysiologydriven drug development of, possibly, more effective treatment ${ }^{5}$.

The role of inflammation in central nervous system (CNS) disease etiopathogenesis has already been widely investigated, and elevated serum inflammatory mediators have been found in many neurological disorders such as cerebral ischemia, multiple sclerosis, Parkinson's disease, Alzheimer's disease, and traumatic brain injury ${ }^{6-11}$. A growing body of evidence now implicates a link between inflammation and different epilepsy syndromes, considering both an inflammatory state in the epileptic brain as well as increased permeability of the blood-brain barrier, leading to enhanced neuronal excitability 12. A clear link between inflammation and seizures is seen in patients with Rasmussen encephalitis, in whom inflammasome activation and associated increased cytokine production was shown ${ }^{13,14}$. In addition, immune system activation is of evident importance in patients with febrile seizures and antibody mediated autoimmune encephalitis (e.g. NMDA-receptor encephalitis) ${ }^{15,16}$. A pro-inflammatory profile has also been found in patients with temporal lobe epilepsy and focal cortical dysplasia, suggesting that even in the absence of evident clinical clues, there may be a link between inflammation and epilepsy ${ }^{17-22}$. Furthermore, immunomodulating therapy with systemic corticosteroids and adrenocorticotropic hormone has proven successful in treating specific epilepsy syndromes, supporting involvement of the immune system in epilepsy ${ }^{23-26}$. 
Inflammation can be studied by measuring inflammatory mediators, such as cytokines, which are soluble proteins that mediate cell growth, activation and differentiation and the inflammatory response ${ }^{27}$. The largest cytokine family comprises the chemokines, which are chemotactic cytokines that induce the migration of immune and non-immune cells and play an important role in the maintenance of CNS homeostatic functions ${ }^{28,29}$. Cytokines are released by immune related cells, such as granulocytes, macrophages and lymphocytes, as well as other cell types, e.g. endothelial and stromal cells. Recent brain studies have shown an important contribution of glial cells (astrocytes and microglia) as well as neurons in the production of pro-inflammatory cytokines ${ }^{30-34}$. Several studies related cytokines (especially $\mathrm{IL}-1 \beta$ ) to changes in neuronal excitability and network characteristics and suggested a potential role for targeted therapy ${ }^{35,36}$.

The aim of this systematic review is to provide an overview of the current evidence for activation of inflammatory mediators (proteins) in epilepsy in humans. We summarize the main outcomes in one overview, and present several meta-analyses comparing levels of inflammatory mediators in patients and controls. Because the role of inflammation may vary per epilepsy syndrome, the studies concerning cytokines in epilepsy are presented in specific syndromes as well.

\section{Methods}

\section{Search strategy}

A systematic literature search was conducted (May 2014) in the PubMed, EMBASE and Cochrane databases using various synonyms for epilepsy and cytokines. The search string was built by three investigators (EEdV, BVdM and FEJ) and reviewed by an experienced librarian. The full search strategy is supplemented as appendix 1.

We included studies of humans with epilepsy or febrile seizures concerning cytokine measurement in serum, cerebrospinal fluid (CSF) or brain tissue. Exclusion criteria were: animal studies, review articles, case reports, treatment studies, genetic or epigenetic studies and acute symptomatic epilepsy (seizures due to acute CNS infection or acute traumatic brain injury).

All titles were screened by a single investigator (EEdV) and the ones that obviously met the exclusion criteria were excluded. All remaining titles and abstracts were independently screened by two investigators (EEdV and BvdM). Both authors independently checked the remaining articles for full-text eligibility. Judgment differences were solved by discussion 
and if no consensus was reached, a third author reviewed the article as well (FEJ). Lastly, reference lists of included articles were checked for missing articles.

\section{Data processing}

Study characteristics and outcomes of all included studies were summarized and are listed in appendix 2, grouped by epilepsy syndrome. A distinction was made for the study design (patients versus controls, postictal versus baseline or in vitro stimulation studies) and the material in which the cytokines were measured (brain tissue, CSF or serum). The total number of epilepsy patients in every single included study was counted to give an estimate of the impact of that particular study.

If means and standard deviations were not presented in the selected publications, corresponding authors of included studies were contacted for additional data for subsequent meta-analyses. 14 authors were contacted, and additional data was provided by two authors. In case insufficient quantitative data was available in the article and no additional data was provided, studies were excluded from meta-analysis. Meta-analyses were conducted for each of the cytokines, with different analyses for serum and CSF, and represented in figure 3 (all individual meta-analyses are provided as supplementary material 1). In case outcomes were significant, subsequent subgroup analyses were performed for all cytokines that were investigated in two or more studies (for serum and CSF separately), and provided as supplementary material 2.

\section{Statistics}

All meta-analyses were performed with Review Manager (RevMan, version 5.3, Copenhagen: The Nordic Cochrane Centre, The Cochrane Collaboration, 2014). Weighted mean differences and 95\% confidence intervals (Cl) were calculated using a random effects model. Heterogeneity was tested for all meta-analyses by a Q test, and $\mathrm{I}^{2}$ was calculated to determine the percentage of variance attributable to heterogeneity.

\section{Results}

\section{Search results}

The search yielded 3.980 articles, of which most were excluded on the basis of title and abstract screening. 149 articles were selected for full-text review, which resulted in a further reduction to 66 articles which were selected for this review. The process of study selection is summarized in figure 1. Study characteristics and outcomes of all included studies are listed in appendix 2. 


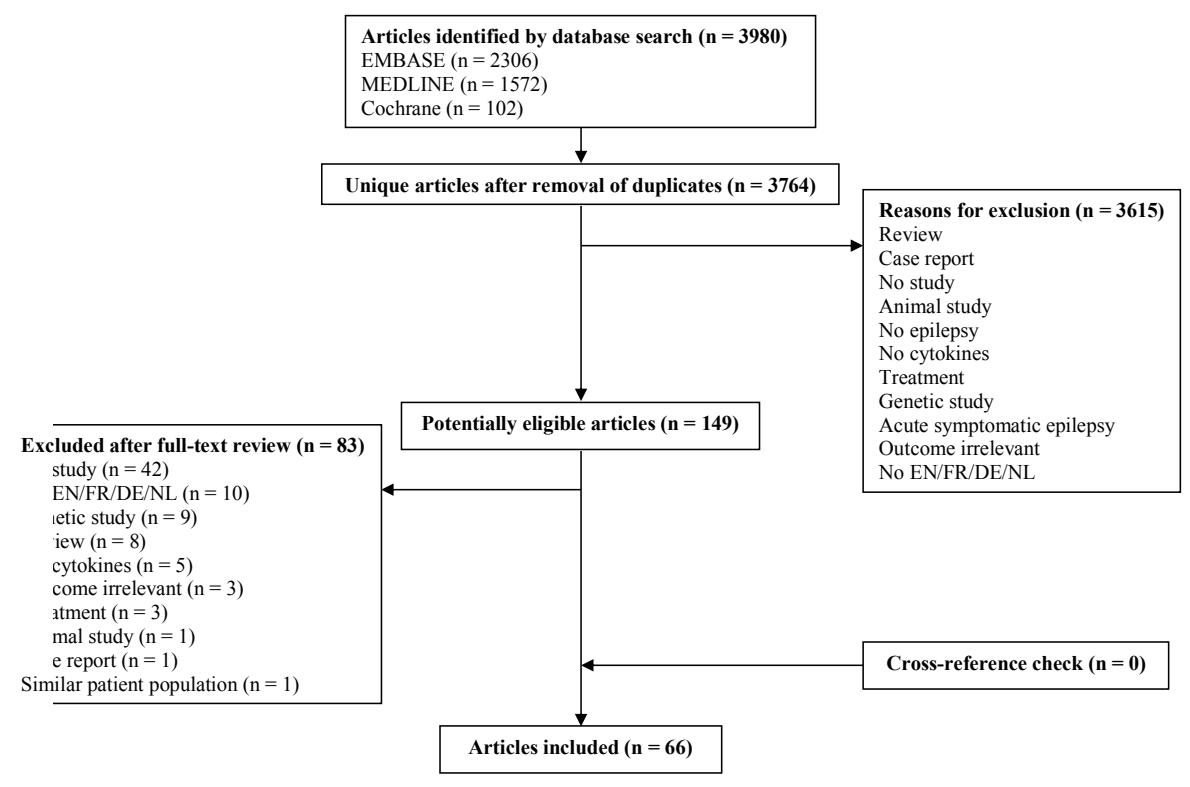

Fig. 1: Flow-chart of search strategy.

\section{Inflammation and epilepsy}

A total of 51 different inflammatory mediators were investigated in the included studies. These studies reported protein levels in serum, CSF or brain tissue and compared patients to controls, or, in a few studies, post-ictal to baseline levels. Figure 2A provides an overview of all studied mediators that were reported to be significantly different in patients versus controls, and the distribution in serum, CSF and brain tissue. These significant findings were found opposed by all studied mediators that were not significantly different between patients and controls, which are represented in figure 2B. Both figures include 57 studies that directly compared patient to control cytokine levels. Nine studies were not included in the graphs: Three compared postictal to baseline levels and all showed a significant increase in IL-6, two studies also compared IL-1ra pre- and postictally and one found a significant increase in IL-1ra (appendix 2) 37-39. Six studies were in vitro peripheral blood mononuclear cell (PBMC) stimulation studies (summarized in paragraph 3.4 and appendix 2). When comparing serum, CSF and brain tissue, a partial overlap is observed in the proteins that were reported to be significantly elevated in epilepsy patients. Several cytokines, such as IL-1 ra, IL-1 $\beta$, IL-6 and CXCL8/IL-8, were reported to be increased in epilepsy patients in all three media, whereas IFN- $\gamma$ and TNF-a were elevated only in serum and CSF. IL-1 a, IL-7 and IL-13, as well as the chemokines CCL2-5, -19 and -22, were increased exclusively in brain tissue. 
A
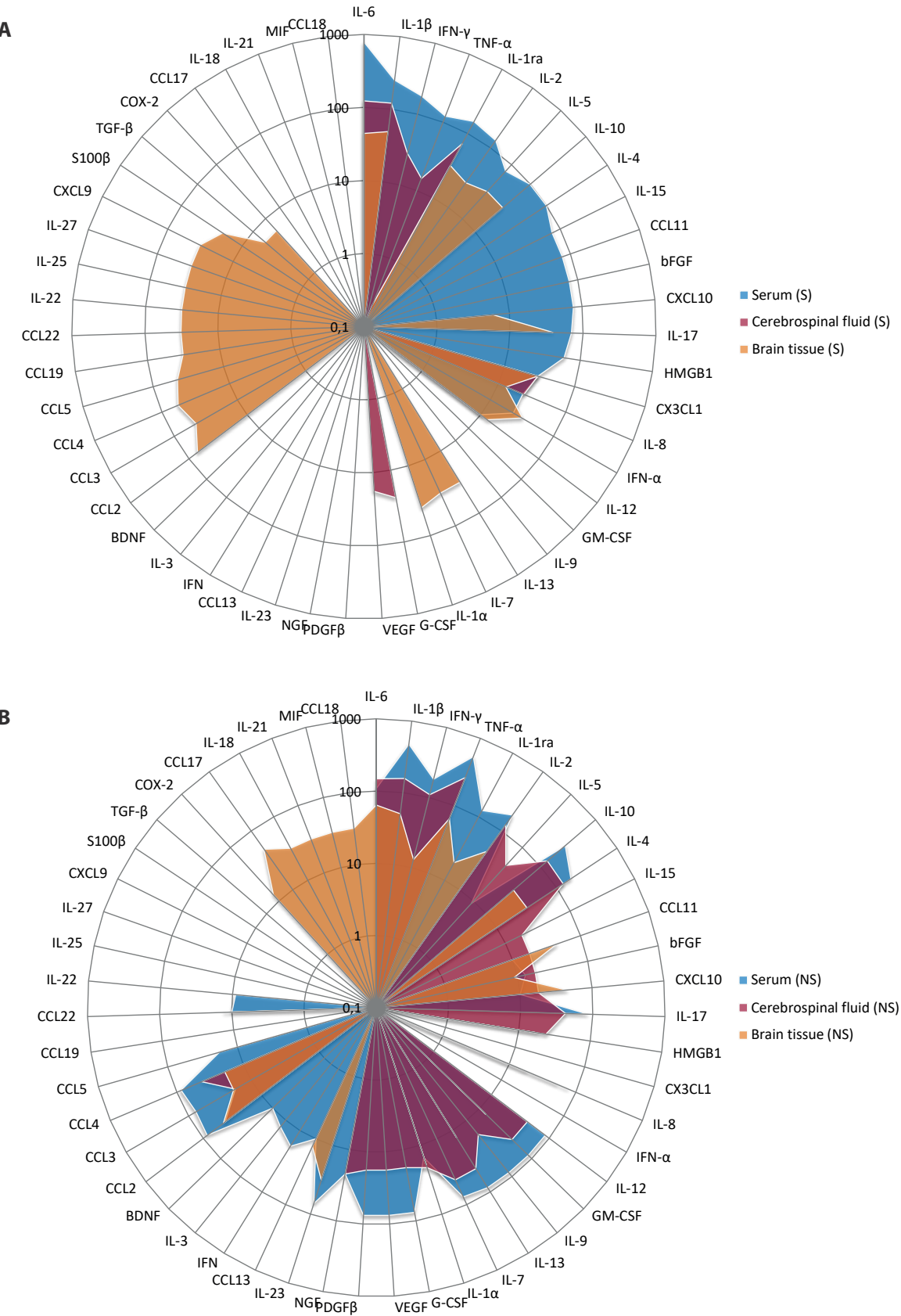

Fig. 2: Radar graphs of the included cytokines, represented separately for serum, cerebrospinal fluid and brain tissue (total number of studies included: 57).

Each axis of the circular radar graph represents one cytokine. The $y$-axis represents the number of patients in the studies. (A) Cytokines that were significantly different (S) between epilepsy patients and controls. (B) Cytokines that were studied but not significantly different (NS) between epilepsy patients and controls. 
Meta-analyses were conducted for all proteins to assess overall significance in serum (fig. 3A) and CSF (fig. 3B). This figure includes both meta-analyses (in case two or more studies investigated a protein), and single study results. In serum, many studies investigated IL-1 $\beta$, IL-6 and TNF-a, of which solely IL- 6 was increased in patients. A meta-analysis of two studies that investigated serum IL-17 showed clearly increased levels in epilepsy patients, with a mean difference of $38 \mathrm{pg} / \mathrm{mL}$ (95\% Cl: 29-47, p<0.001), which is a 5-15 fold increase compared to normal individuals ${ }^{40}$. Single study results reported in the meta-analysis overview (studies reporting mean and SD) revealed a significant elevation of serum IL-1a, IFN-a, CCL4, CCL11, CXCL10, CX3CL1, HMGB1 and bFGF in epilepsy patients. In CSF, numerous studies investigated IL-1 $\beta, I L-6$ and TNF-a, of which solely IL-1 $\beta$ was marginally elevated. A meta-analysis including two studies showed a significant elevation of CSF IL10 in patients. Increased levels in CSF of the cytokines IL-12, IL-13 and CX3CL1 were reported in single studies.

A

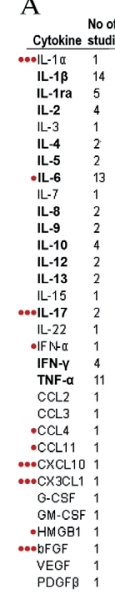

\begin{tabular}{ll} 
No of & No \\
studies pati \\
\hline 1 & 15 \\
14 & 366 \\
5 & 184 \\
4 & 10 \\
1 & 13 \\
2 & 85 \\
2 & 84 \\
13 & 42 \\
1 & 76 \\
2 & 89 \\
2 & 85 \\
4 & 139 \\
2 & 85 \\
2 & 85 \\
1 & 76 \\
2 & 85 \\
1 & 9 \\
1 & 10 \\
4 & 139 \\
11 & 29 \\
1 & 76 \\
1 & 76 \\
1 & 76 \\
1 & 76 \\
1 & 76 \\
1 & 43 \\
1 & 76 \\
1 & 76 \\
1 & 41 \\
1 & 76 \\
1 & 76 \\
1 & 76 \\
& \\
&
\end{tabular}
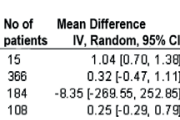
$1.04[0.70,1.38]$ $0.32[-0.47,1.11]$ $-269.55,252.85]$
$0.25[-0.29,0.79]$ $0.83[-0.42,2.08]$ $8.13 \mid-21.65,37.91$
$12.37[-1673,41.47$ $2.37[-16.73,41.47]$
$3.11[1.14,5.08]$ $3.11[1.14,5.08]$
$3.32[-0.07,6.71]$ $3.32[-0.07,6.71]$
$31.32[-52.43,115.07]$ $32.69[-140.71,326.09]$ $13.91[-42.61,70.43]$ $2.73 \vdash-155.14,360.60]$ $18.38[-25.55,62.31]$ $6.20[-4.15,16.55]$ $38.09[29.37 .46 .81]$
$283.76+-109.63,677.15]$
$0.27[0.12,0.42]$ $0.27[0.12,0.42]$
$0 .-45.93,85.33]$ $9.70[-45.93,05.33)$
$0.59+-1.51,2.69$ $0.59[-1.51,2.69]$
$4.33[-19.52,48.18]$
$1.54[-1.45,4.5]$ $4.33[-19.52,48.18]$
$1.54[-1.45,4.53]$ $1.90[0.19,203.61]$ $30.20[6.01,54.39$ 639.71 [292.24, 987.79$]$
$730.00[402.15,1057.85]$ $9.77[-1,10,20.64]$
$40.58[-60.32,141.48]$ $78[-60.32,141.48]$
$7.80[0.15,15.45]$
$21.30[10.45,32.15$ $21.30[10.45,32.15$
$57.42[-25.98,140.82]$ $57.42[-25.98,140.82]$
$26.75[-2746.61,4600.11]$

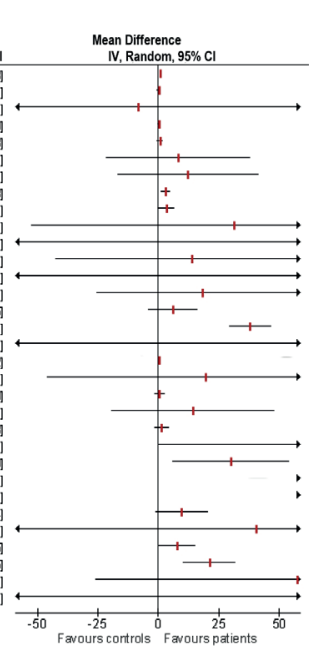

B

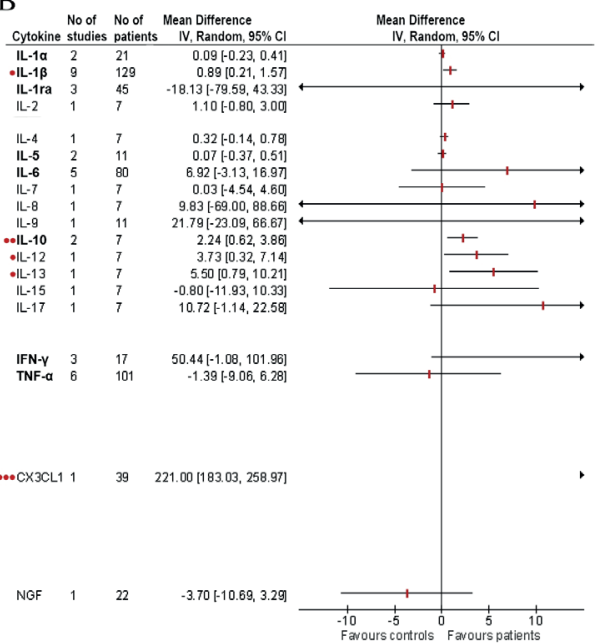

\section{Fig. 3:}

(A) Overview of all meta-analyses of cytokine concentrations in serum of epilepsy patients versus controls.

(B) Overview of all meta-analyses of cytokine concentrations in cerebrospinal fluid of epilepsy patients versus controls. Reported are both meta-analyses (in case two or more studies investigated a protein), and single study results. Mean difference is in $\mathrm{pg} / \mathrm{mL}$. The dots mark the significantly different cytokines: ${ }^{*}=$ $p<0.05,{ }^{* *}=p<0.01,{ }^{* * *}=p<0.001$, and are represented for both meta-analyses and single study results.

\section{Specific subtypes of epilepsy}

\section{Temporal lobe epilepsy (TLE)}

In brain tissue of TLE patients, the IL-1 family was most extensively studied. IL-1 a levels were shown to be higher in epilepsy patients than in non-epilepsy controls ${ }^{17,41}$, irrespective of the presence of hippocampal sclerosis ${ }^{8,17}$. Increased IL-1 $\beta$ expression was found in TLE 
patients when compared to autopsy controls ${ }^{42}$, especially in patients with hippocampal sclerosis ${ }^{43,44}$. Of the CCL-chemokine family, CCL3, -4 and -5 were found to be higher in TLE tissue when compared to autopsy controls ${ }^{17}$, which is confirmed by another study that found CCL3, -4 and -5 expression in TLE neurons, contrary to control neurons ${ }^{42}$. Baseline serum levels of IL-1ra have been found to be lower compared to normal population levels in one study ${ }^{39}$, whereas other studies failed to show a difference ${ }^{45,46}$. In contrast to findings in brain tissue, IL-1 $\beta$ levels in serum of TLE patients were not elevated when compared to healthy controls ${ }^{45-48}$. However, a decrease in IL-1 ra/IL-1 $\beta$ ratio was reported after a seizure, which leads to increased influence of the pro-inflammatory IL-1 $\beta$ and may implicate a pro-inflammatory state in the brain ${ }^{39,47}$. A meta-analysis of serum IL-6 levels in TLE patients revealed marginal but significant IL-6 elevation when compared to controls (supplementary material 2). Serum IL-6 levels were significantly elevated in five out of eight studies $37,39,46,48,49$, while two small studies showed a trend towards significance ${ }^{45,47}$. Thus, in temporal lobe epilepsy patients, levels of pro-inflammatory cytokines from the IL-1 family and CCL3-5 were increased in brain tissue, and IL-6 levels were increased solely in serum.

\section{Other epilepsy etiologies}

In contrast to findings in temporal lobe epilepsy, only one out of five studies demonstrated elevated IL-1 $\beta$ levels in cerebrospinal fluid of febrile seizure patients ${ }^{50}$. In serum, three out of ten studies demonstrated elevated IL-1 $\beta$ levels within 12 hours after a febrile seizure (which returned to baseline after three months ${ }^{51-53}$ ). Meta-analyses of IL-1 $\beta$ in CSF and serum of febrile seizure patients revealed no significant differences. Serum IL-1 ra levels were investigated in one study and shown to be higher in febrile seizure patients ${ }^{54}$. Interestingly, this study showed an increase in IL-1 ra/IL-1 $\beta$ ratio, whereas in the TLE studies this ratio was found to be decreased. IL-6 levels were elevated in CSF of patients in two out of five studies ${ }^{55,56}$. A meta-analysis of IL-6 levels in serum of febrile seizure patients, including merely four studies reporting mean and SD, revealed no significant serum IL-6 elevation, despite the observation of elevated IL-6 levels in serum in five out of seven studies ${ }^{52-55,57}$.

Studies exploring levels of inflammatory mediators in patients with West syndrome are scarce, and results are equivocal. Regarding IL-1 levels, in patients IL-1 ra has been found to be decreased in CSF ${ }^{58}$, while another study demonstrated a significant increase of IL$1 \mathrm{ra}$ in serum ${ }^{59}$. One study investigated levels of inflammatory proteins in other epileptic encephalopathies and demonstrated increased serum levels of IL-6 in patients with Lennox-Gastaut syndrome and electrical status epilepticus in sleep (ESES) syndrome ${ }^{60}$. An increase in IL-6 expression was also shown in resected brain tissue of patients with focal cortical dysplasia (FCD) and tuberous sclerosis ${ }^{61}$. In FCD and glioneuronal tumors 
Chapter 3. Inflammatory mediators in human epilepsy

$\mathrm{IL}-1 \beta$ and its receptor were found to be highly upregulated, while relative paucity of their antagonists was reported ${ }^{21}$.

\section{In vitro stimulation studies}

Additional evidence for an immune related compound in epilepsy comes from several studies showing that PBMCs of epilepsy patients produced increased amounts of inflammatory mediators of the IL-1 family (significant in four of six studies investigating this cytokine family), IL-6 (significant in two of two studies), IL-10 (significant in two of two studies) and IFN-a (significant in the single study investigating this cytokine) upon in vitro stimulation, compared to PBMCs of controls. PBMCs of patients and controls were stimulated with lipopolysaccharide (LPS) in four studies, ds-RNA of a common viral vector in one study and mitogen in one study (appendix 2) 62-67. Thus, stimulation of immune related cells generates a more prominent immune response in patients with epilepsy, which may indicate a "hyper-reactive immune state".

\section{Discussion}

The pathophysiology of epilepsy is incompletely understood. Accumulating evidence suggests a link between epilepsy and inflammation. With this systematic review and meta-analysis we created an overview of the current evidence for the involvement of inflammatory mediators in epilepsy patients.

There is some overlap between the findings for inflammatory proteins in serum, CSF and brain tissue as shown in the radar graphs (fig. 2A). Many studies found significantly elevated IL-6 levels in both serum and cerebrospinal fluid of epilepsy patients with different underlying etiologies. IL-1 $\beta$ levels have been found elevated in brain tissue and CSF of epilepsy patients, and chemokine levels were predominantly found elevated in studies investigating brain tissue. However, the results of some individual studies are conflicting. Single studies may provide relatively solid evidence, only if adequately powered and if their results are strongly significant.

We performed meta-analyses combining all studies in serum or CSF providing sufficient quantitative data to overcome small sample sizes of individual studies that may have led to insignificant results, or the opposite, to an over interpretation of the differences found. Meta-analyses combining results of all epilepsy patients together revealed a significant increase of IL-6 and IL-17 in serum and IL-1 $\beta$ and IL-10 in CSF. Etiology specific metaanalyses revealed elevated IL-6 levels in temporal lobe epilepsy patients. 
These findings provide strong support for the involvement of inflammation in patients with epilepsy. Pro-inflammatory cytokines such as IL-1, IL-6 and IL-17 were widely found to be increased, while many studies also reported a significant increase in TNF-a. The differences in findings between serum, CSF and tissue may reflect a differential involvement of components of the inflammatory process. We hypothesize that some inflammatory mediators (particularly IL-6) can be seen as general markers of immune system activation (both local and systemic), while many chemokines (predominantly found elevated in brain tissue), such as several members of the CCL-family and CX3CL1, may be involved in focal cerebral inflammation. Several studies comparing CSF and serum levels within the same patients have been published, with inconsistent findings. Their interpretation is uncertain considering large differences in turnover between different cytokines, which inherently can result in differences between tissue, CSF and serum cytokine levels 27,45,68,69. Previous studies demonstrated that, under physiological conditions, chemokines are synthesized by neurons as well as by macrophages and glial cells, suggesting a role in the regulation of processes in the homeostatic brain by acting as neurotransmitters ${ }^{70-72}$. Under pathological inflammatory conditions, chemokine production is enhanced and these cytokines are synthesized in activated microglial cells and astrocytes, emphasizing their role in the brain's protection mechanisms ${ }^{73}$. However, many inflammatory proteins are known to be transported across the blood-brain barrier ${ }^{74-77}$ and in addition disruption of the blood-brain barrier is often seen in patients with epilepsy ${ }^{78}$. Interestingly, IL-17 was found to be increased in the current meta-analysis and it is known that IL-17 attracts monocytes and neutrophils and can cause blood-brain barrier disruption ${ }^{79-81}$. In addition, IL-22 (produced by T helper cells just like IL-17) was found elevated in brain tissue and IL22 is another cytokine that can promote blood-brain barrier disruption. ${ }^{81}$. In conclusion, it remains unclear whether increased cytokine levels in plasma and CSF of epilepsy patients relate to a cerebral inflammatory process alone, or might also arise from postictal peripheral muscular recovery or circulating immune cells.

Although mainly pro-inflammatory cytokines were found increased in patients with epilepsy, we also found increased IL-10 in CSF. IL-10 has an important regulatory, antiinflammatory function but the mechanisms that lead to $I L-10$ production during infection and inflammation are incompletely understood. IL-10 counteracts many proinflammatory processes during infection as well as in autoimmune disorders. It may ameliorate immunopathology by preventing inflammation associated tissue damage 82. We hypothesize that the increase of IL-10 in CSF of epilepsy patients can be due to counteracting mechanisms to the pro-inflammatory stimuli. 
Chapter 3. Inflammatory mediators in human epilepsy

The role of inflammation in patients with epilepsy has been widely debated. It could be considered as just an epiphenomenon, resulting from seizure-induced damage to the brain or from excessive muscular activity. Strenuous exercise can induce increased production of pro- and anti-inflammatory cytokines in serum 83 and the occurrence of (convulsive) seizures may result in a comparable muscular exhaustion and cytokine response. Furthermore, in vitro stimulation studies showed increased production of IL-1 $\beta$, IL-6 and IL-10 by PBMCs in epilepsy and febrile seizure patients, possibly suggesting a systemic instead of focal cerebral origin of these cytokines. Interestingly, the response on pro-inflammatory stimuli is much more profound in PBMCs of epilepsy patients compared to PBMCs of healthy controls 62-66. However, it seems unlikely that only these "secondary" immune activating mechanisms declare the pro-inflammatory profile of patients with epilepsy. Both clinical and experimental findings support a more causative role:

Clinical studies have shown the potential of corticosteroids and possibly also immunoglobulins (IVIG) as anti-seizure treatment in pediatric epilepsy syndromes. Treatment of infantile spasms with corticosteroids or adrenocorticotrophic hormone (ACTH) has become standard care based on a Cochrane review of randomized controlled trials (RCT) ${ }^{26}$. The use of corticosteroids for other epilepsy syndromes, especially for electrical status epilepticus in sleep (ESES) has not been evaluated in RCTs ${ }^{84}$, but is based on remarkable results reported in case series $24,25,85,86$. IVIG is commonly used in pediatric epilepsy patients and has been reported to be effective in adult epilepsy patients. Findings have been mixed but are overall promising for the use of IVIG ${ }^{87,88}$. In addition, treatments linked to immunomodulation have been shown effective in the treatment of epilepsy: Vagal nerve stimulation and ketogenic diet decrease seizure burden and other symptoms in patients with (medically refractory) epilepsy ${ }^{89-94}$.

Also, a very common precipitant of seizures in children under the age of six is fever, which is part of inflammatory responses. The occurrence of (multiple) febrile seizures predisposes to temporal lobe epilepsy ${ }^{95,96}$. Experimental studies have provided evidence for an important role of IL-1 $\beta$ as a link between febrile seizures and epileptogenesis 16,97,98. Another study has shown that administration of IL-1 $\beta$ can worsen seizure activity and lower seizure threshold in rats and mice ${ }^{99,100}$, which has also been reported for IL-6 101 and lipopolysaccharide, a potent inducer of inflammation ${ }^{102,103}$. Furthermore, postnatal injection of IL-1 $\beta$ in a rat model of febrile seizures enhanced adult seizure susceptibility and hippocampal neuronal cell loss ${ }^{97}$, which could also implicate a mechanism behind the relationship between prolonged febrile seizures and the occurrence of temporal lobe epilepsy. Finally, early raise of IL-1 1 levels preceded seizure onset in pilocarpine-treated rats, suggesting that elevated IL-1 $\beta$ levels were not the result of seizure activity ${ }^{104}$. 
The use of more targeted immunomodulating therapy may be considered. Cytokinemediated therapy is widely used in several autoimmune disorders like rheumatoid arthritis, inflammatory bowel disease and multiple sclerosis ${ }^{105,106}$. Cytokine inhibitors have been investigated as a possible treatment for epilepsy in animal studies. A recombinant IL-1 receptor antagonist has demonstrated to be capable of significantly reducing seizure duration and recurrence 100,107,108, and incidence of status epilepticus in rodents ${ }^{104}$. Also, losartan, which is an angiotensin II type 1 antagonist that was identified as a blocker of TGF- $\beta$ signaling, has shown to prevent the development of seizures in a rat vascular brain injury model ${ }^{109}$ and in kainate-induced epilepsy in rats ${ }^{110}$. Furthermore, administration of an antagonist of the pro-inflammatory mediator HMGB1 significantly delayed the seizure onset and diminished the frequency and duration of kainate-induced seizures in mice ${ }^{111}$. Micro-RNAs have also been suggested as a potential target for therapy. Recent studies found evidence that micro-RNAs can control microglial activity and thereby modify the inflammatory profile and possibly also influence the related changes in neuronal architecture $33,12,113$.

In humans, only one specific cytokine inhibitor has been investigated as add-on therapy in a phase II trial. VX-765 is a selective inhibitor of interleukin-converting enzyme, thereby inhibiting release of IL-1 ${ }^{114}$. In a short-duration randomized placebo-controlled trial of 60 patients with pharmacoresistant focal epilepsy, there was no significant difference in seizure rate or $50 \%$ responder rate after six weeks, although a trend could be noted ${ }^{115}$. However, post-hoc analyses revealed a stronger effect of VX-765 later in the treatment period. Furthermore, after six weeks $12.5 \%$ of VX-765 subjects were seizure free versus $0 \%$ of patients in placebo group. A follow-up study was terminated early due to administrative reasons and lacked the power for statistical comparisons ${ }^{116}$.

Based on the results of this systematic review and meta-analysis, we suggest future immunomodulating therapy may be directed at inhibiting a common intracellular proinflammatory pathway. Many pro-inflammatory cytokines, such as IL-1 $\beta, I L-6$ and TNF-a, are produced after activation of nuclear factor- $\mathrm{kB}$, a nuclear transcription factor which controls many genes involved in inflammation. An important activator of this intracellular pathway is HMGB1, which binds, amongst others, to toll-like receptor 4 (TLR4) $34,111,117$. Thus, inhibition of HMGB1 and TLR4 may result in a decrease of the pro-inflammatory state that was found in patients with epilepsy.

Several aspects had to be challenged in this study. The overview in figure 2 is probably an underestimation of the role of inflammatory mediators in epilepsy, as several studies were based on a small data set due to rarity of the diseases. Moreover, differences in study design and technical or patient related (e.g. age, gender) inequalities could have 
influenced study outcomes. Only an individual patient data meta-analysis would allow analysis of these characteristics in relation to cytokine levels. Also, we did not include studies reporting on micro-RNAs in the current systematic review and meta-analysis. During recent years, micro-RNAs have been shown to be important regulators of gene expression at the post-transcriptional level ${ }^{118,119}$. Although some micro-RNAs are thought to regulate the inflammatory response, many micro-RNAs regulate the expression of noninflammatory genes and we considered micro-RNAs beyond the scope of this systematic review and meta-analysis. Despite these challenges, this systematic review provides a complete overview of inflammatory mediators in human epilepsy. We described the results of individual studies and the addition of several meta-analyses strengthens our results and can reveal clues from accumulating evidence that were not significant in individual studies.

In conclusion, evidence has accumulated for the presence of an inflammatory cytokine profile in epileptogenesis. We hypothesize that these proteins fulfill a role in the cascade of inflammation, blood-brain barrier disruption, increased excitability and, finally, epileptogenesis. Targeting those inflammatory pathways with specific cytokine inhibitors is a challenging next step in the treatment of refractory epilepsies, and should be explored in further clinical studies.

\section{Acknowledgements}

We thank dr. Takashi Shiihara of the department of Neurology, Gunma Children's Medical Center, Japan, and dr. Yukitoshi Takahashi and dr. Jun Mine of the department of Pediatrics, National Epilepsy Center, Shizuoka Institute of Epilepsy and Neurological Disorders, Japan, for providing us with additional data.

\section{Disclosure}

This work was supported by grants from the Dutch Epilepsy Fund and the Wilhelmina Children's Hospital Research Fund. The funding sources had no involvement in this study. WdJ is supported by Understanding Childhood Arthritis Network (UCAN) and Center for Translational Molecular Medicine (CTMM, TRACER). 


\section{Appendices}

Appendix 1: Search strategy in PubMed, EMBASE and Cochrane databases (subsequent rows were linked by "OR")

\begin{tabular}{|c|c|}
\hline Domain & AND Determinant \\
\hline Epilepsy & Cytokine \\
\hline Seizure & Chemokine OR CCL OR XCL \\
\hline Febrile convulsion & Intercrine \\
\hline $\begin{array}{l}\text { Landau Kleffner OR (acquired aphasia AND convulsive disorder) } \\
\text { OR acquired auditory verbal agnosia }\end{array}$ & Interleukin OR IL- OR (IL1 to IL9) \\
\hline $\begin{array}{l}\text { Infantile spasms OR West syndrome OR hypsarrhythmia OR } \\
\text { (salaam AND (attack OR seizure)) OR spasmus nutans }\end{array}$ & Interferon OR IFN \\
\hline Ohtahara & Lymphokine \\
\hline Early myoclonic encephalopathy & Monokine \\
\hline Dravet syndrome & (Leukocyte OR macrophage) migration inhibitory factor \\
\hline Myoclonic status in nonprogressive encephalopathies & Lymphotoxin \\
\hline \multirow[t]{12}{*}{ Lennox-Gastaut syndrome } & Macrophage activating factor \\
\hline & Immunologic suppressor factor OR suppressor T-cell factor \\
\hline & Transfer factor \\
\hline & Tumor necrosis factor OR TNF \\
\hline & TGF-beta superfamily proteins OR TGF \\
\hline & Bone morphogenetic protein \\
\hline & Growth differentiation factor \\
\hline & HMGB1 \\
\hline & Heparin-binding protein OR amphoterin \\
\hline & $\begin{array}{l}\text { ICAM OR intercellular adhesion molecule OR cluster of } \\
\text { differentiation } 54 \text { OR CD54 }\end{array}$ \\
\hline & $\begin{array}{l}\text { VCAM OR (vascular cell adhesion AND (protein OR molecule)) OR } \\
\text { cluster of differentiation } 106 \text { OR CD106 }\end{array}$ \\
\hline & VEGF OR vascular endothelial growth factor \\
\hline
\end{tabular}




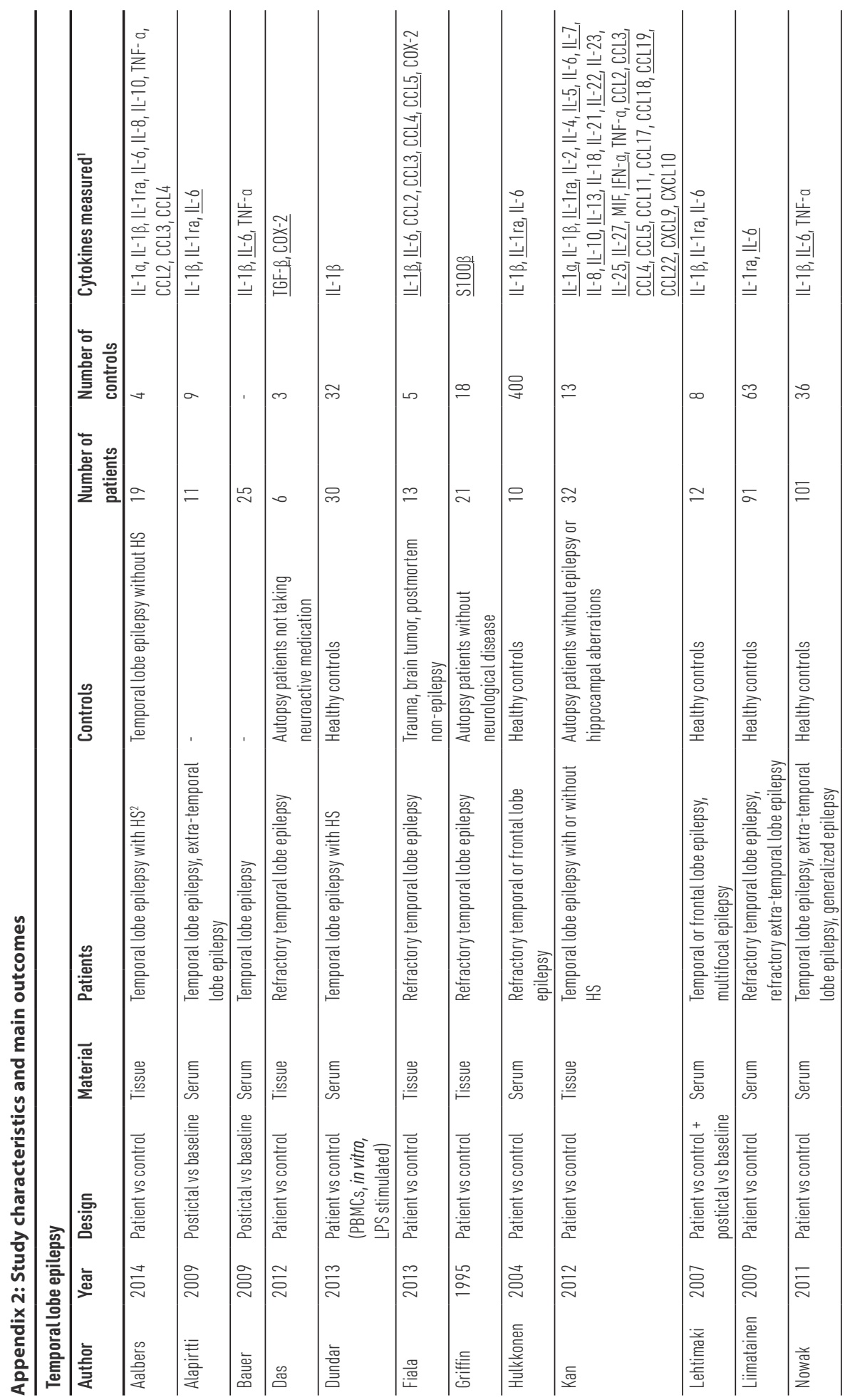




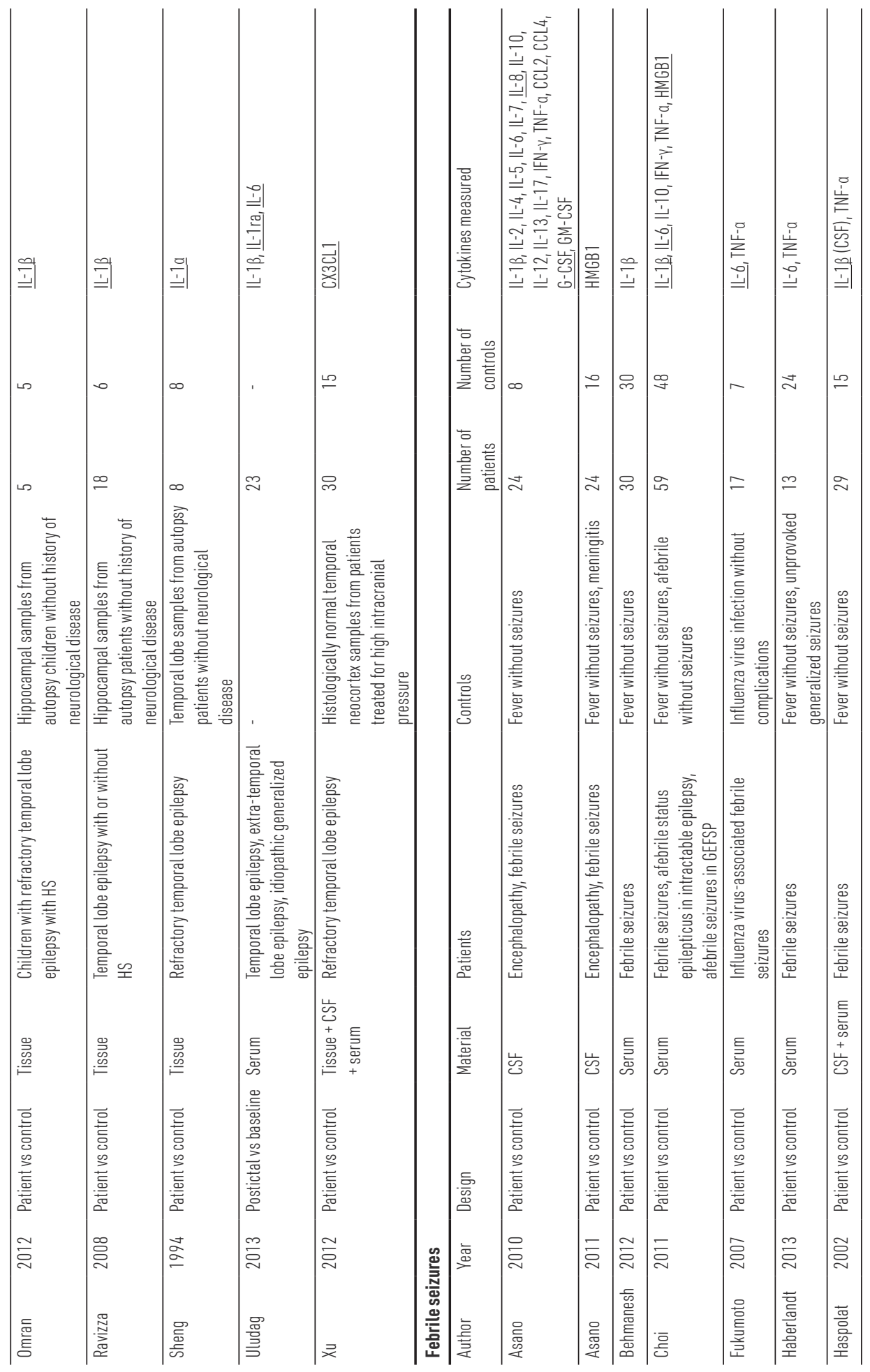




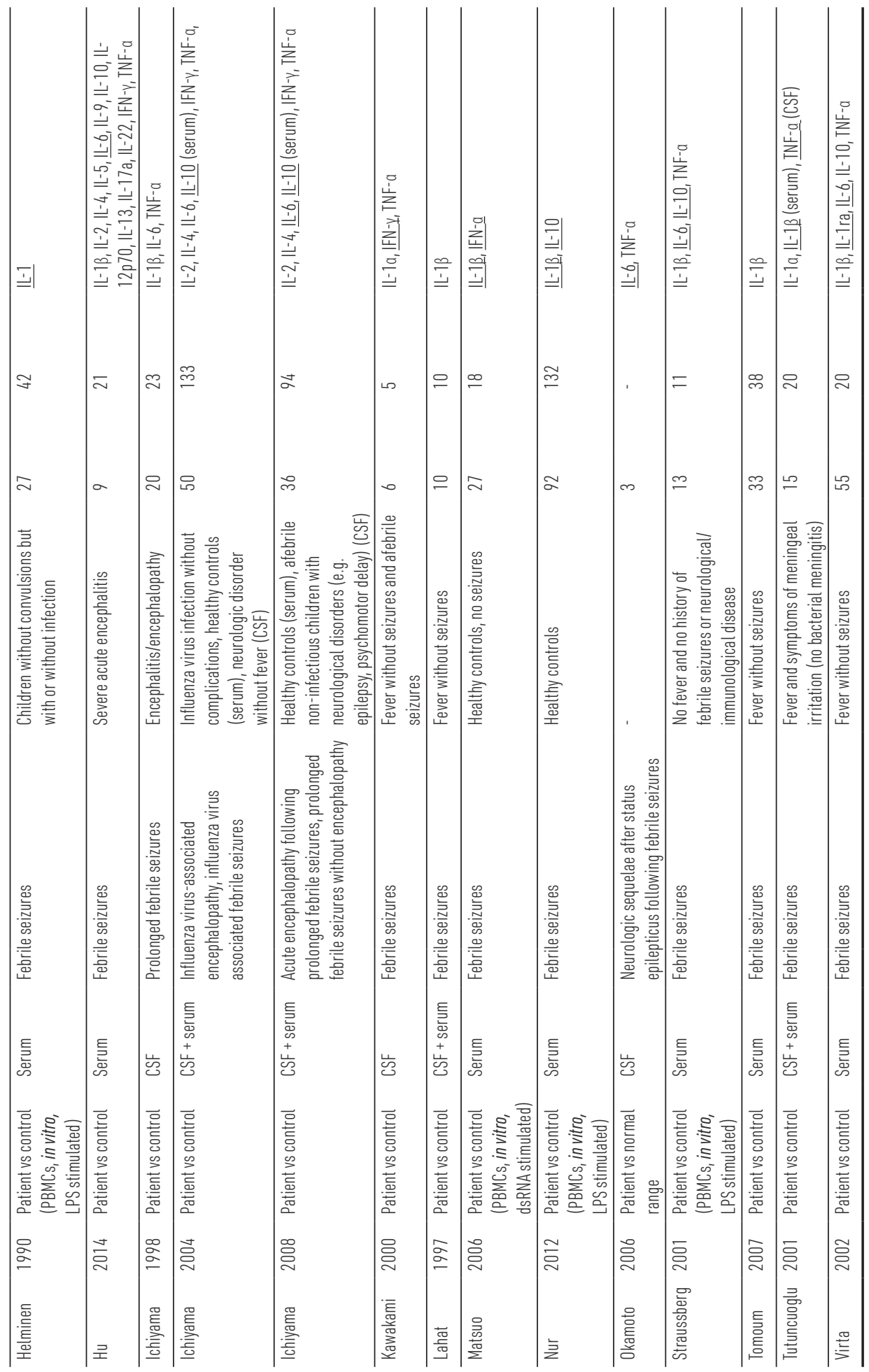




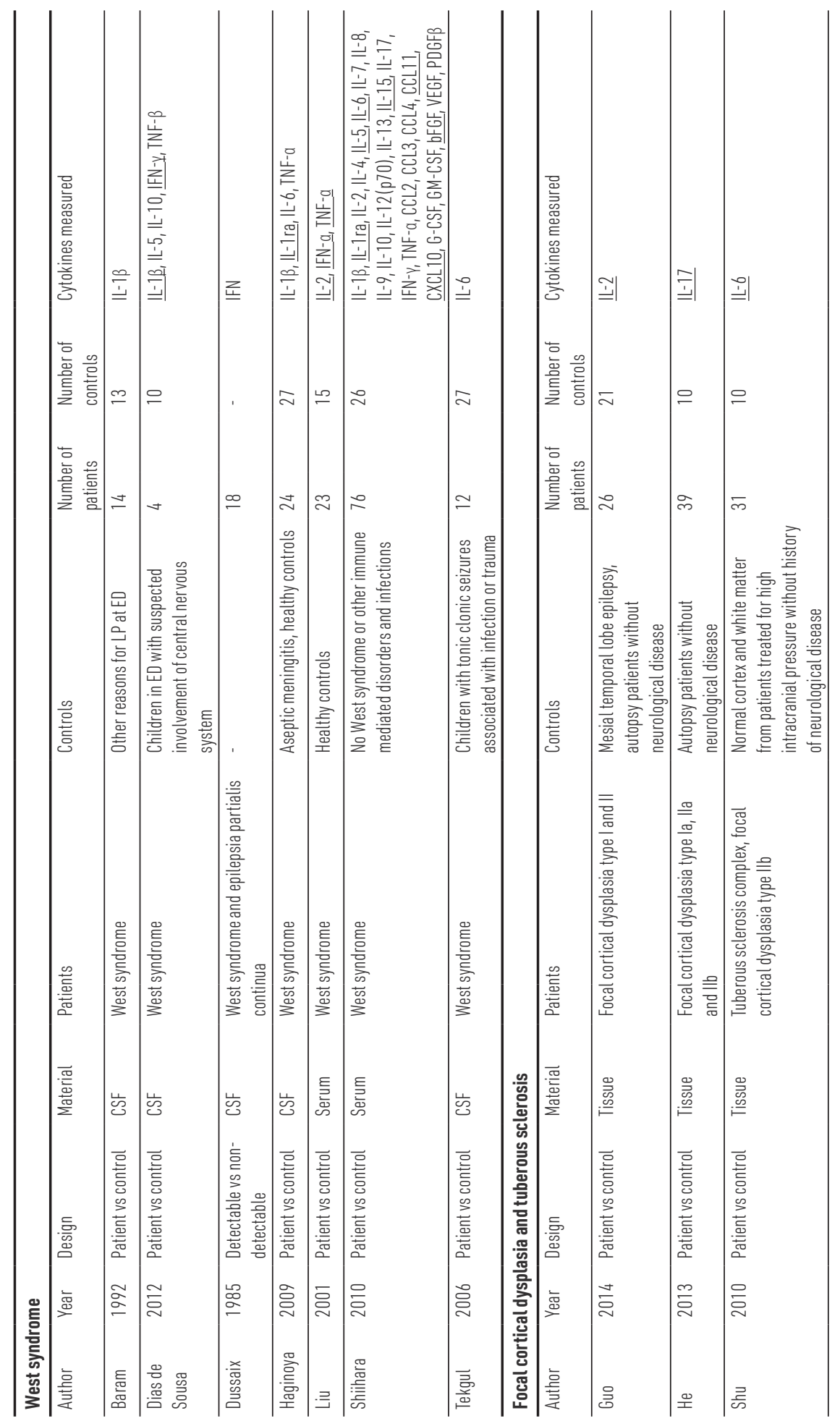


Chapter 3. Inflammatory mediators in human epilepsy

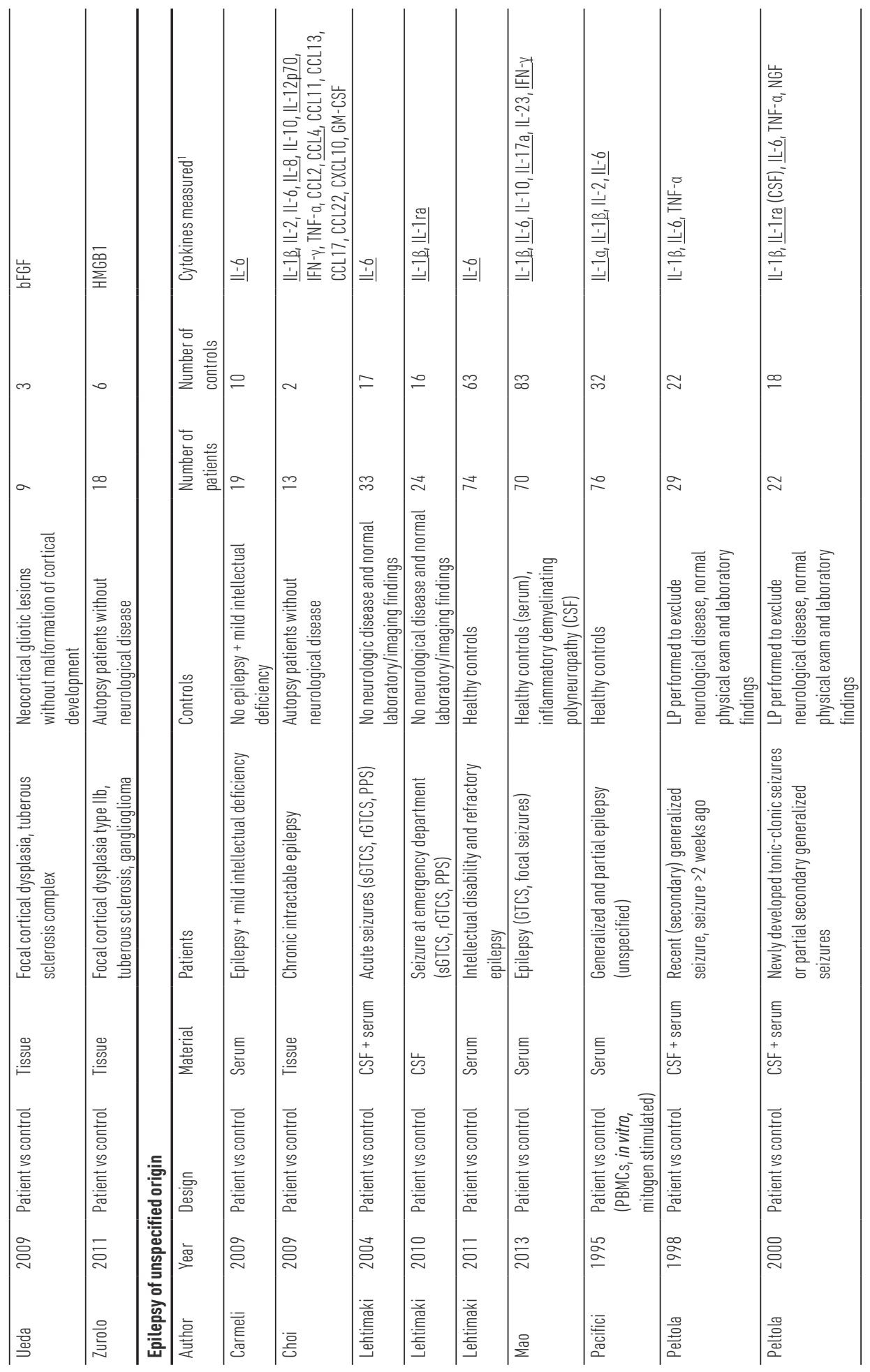




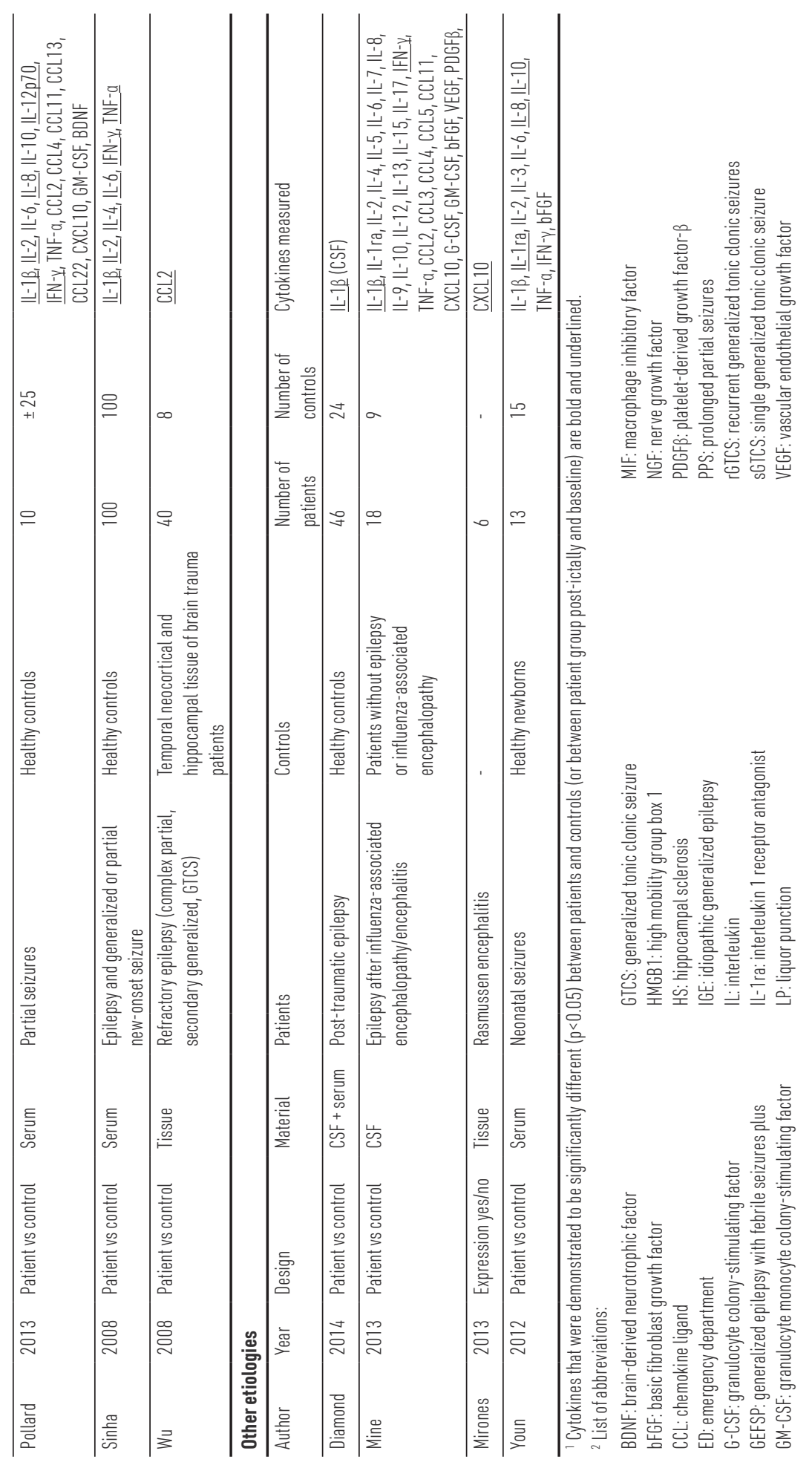


Chapter 3. Inflammatory mediators in human epilepsy

\section{References}

1. Fisher RS, Acevedo C, Arzimanoglou A, et al. ILAE Official Report: A practical clinical definition of epilepsy. Epilepsia. 2014;55(4):475-482. doi:10.1111/epi.12550.

2. Berg AT, Berkovic SF, Brodie MJ, et al. Revised terminology and concepts for organization of seizures and epilepsies: Report of the ILAE Commission on Classification and Terminology, 2005-2009. Epilepsia. 2010;51(4):676-685. doi:10.1111/j.1528-1167.2010.02522.

3. Berg AT, Scheffer IE. New concepts in classification of the epilepsies: Entering the 21st century. Epilepsia. 2011;52(6):1058-1062. doi:10.1111/j.1528-1167.2011.03101.

4. Kwan P, Brodie MJ. Clinical trials of antiepileptic medications in newly diagnosed patients with epilepsy. Neurology. 2003;60(11 Suppl 4):S2-12.

5. Perucca E, French J, Bialer M. Development of new antiepileptic drugs: challenges, incentives, and recent advances. Lancet Neurol. 2007;6(9):793-804. doi:10.1016/S1474-4422(07)70215-6.

6. Perry $\mathrm{VH}$. The influence of systemic inflammation on inflammation in the brain: implications for chronic neurodegenerative disease. Brain Behav Immun. 2004;18(5):407-413. doi:10.1016/j. bbi.2004.01.004.

7. Perry VH, Nicoll J a R, Holmes C. Microglia in neurodegenerative disease. Nat Rev Neurol. 2010;6(4):193201. doi:10.1038/nrneurol.2010.17.

8. Aalbers MW, Rijkers K, Majoie HJ, et al. The influence of neuropathology on brain inflammation in human and experimental temporal lobe epilepsy. J Neuroimmunol. 2014. doi:S0165-5728(14)00095-2.

9. Corps KN, Roth TL, McGavern DB. Inflammation and neuroprotection in traumatic brain injury. JAMA Neurol. 2015;72(3):355-362. doi:10.1001/jamaneurol.2014.3558.

10. Mallucci G, Peruzzotti-Jametti L, Bernstock JD, Pluchino S. The role of immune cells, glia and neurons in white and grey matter pathology in multiple sclerosis. Prog Neurobiol. 2015;127:1-22. doi:10.1016/j. pneurobio.2015.02.003.

11. Hemmer B, Kerschensteiner $M$, Korn T. Role of the innate and adaptive immune responses in the course of multiple sclerosis. Lancet Neurol. 2015;14(4):406-419. doi:10.1016/S1474-4422(14)70305-9

12. Vezzani AM. Preventing epileptogenesis: Antiinflammatory strategy. Epilepsia. 2010;51:166.

13. Ramaswamy V, Walsh JG, Sinclair DB, et al. Inflammasome induction in Rasmussen's encephalitis: cortical and associated white matter pathogenesis. J Neuroinflammation. 2013;10(1):152. doi:10.1186/1742-2094-10-152.

14. Rasmussen T, Olszewski J, Lloydsmith D. Focal seizures due to chronic localized encephalitis. Neurology. 1958;8(6):435-445.

15. Dalmau J, Gleichman AJ, Hughes EG, et al. Anti-NMDA-receptor encephalitis: case series and analysis of the effects of antibodies. Lancet Neurol. 2008;7:1091-1098. doi:10.1016/S1474-4422(08)70224-2.

16. Dubé C, Vezzani A, Behrens M, Bartfai T, Baram TZ. Interleukin-1 $\beta$ contributes to the generation of experimental febrile seizures. Ann Neurol. 2005;57(1):152-155. doi:10.1002/ana.20358.

17. Kan $A A$, de Jager $W$, de Wit $M$, et al. Protein expression profiling of inflammatory mediators in human temporal lobe epilepsy reveals co-activation of multiple chemokines and cytokines. J Neuroinflammation. 2012;9:207.

18. van Vliet E a., Aronica E, Gorter J a. Role of blood-brain barrier in temporal lobe epilepsy and pharmacoresistance. Neuroscience. 2014;277:455-473. doi:10.1016/j.neuroscience.2014.07.030.

19. Henshall DC, Clark RSB, Adelson PD, Chen M, Watkins SC, Simon RP. Alterations in bcl-2 and caspase gene family protein expression in human temporal lobe epilepsy. Neurology. 2000;55(2):250-257. doi:10.1212/WNL.55.2.250.

20. Tan C-C, Zhang J-G, Tan M-S, et al. NLRP1 inflammasome is activated in patients with medial temporal lobe epilepsy and contributes to neuronal pyroptosis in amygdala kindling-induced rat model. J Neuroinflammation. 2015;12(1):1-12. doi:10.1186/s12974-014-0233-0.

21. Ravizza T, Boer K, Redeker S, et al. The IL-1b system in epilepsy-associated malformations of cortical development. Neurobiol Dis. 2006;24(1):128-143. doi:10.1016/j.nbd.2006.06.003. 
22. Van Gassen KLI, De Wit M, Koerkamp MJ a G, et al. Possible role of the innate immunity in temporal lobe epilepsy. Epilepsia. 2008;49(6):1055-1065. doi:10.1111/j.1528-1167.2007.01470.

23. Gupta R, Appleton R. Corticosteroids in the management of the paediatric epilepsies. Arch Dis Child. 2005;90(4):379-384. doi:10.1136/adc.2004.051375.

24. Buzatu M, Bulteau C, Altuzarra C, Dulac O, Van Bogaert P. Corticosteroids as treatment of epileptic syndromes with continuous spike-waves during slow-wave sleep. Epilepsia. 2009;50:68-72. doi:10.1 111/j.1528-1167.2009.02224.

25. van den Munckhof B, van Dee V, Sagi L, et al. Treatment of electrical status epilepticus in sleep: A pooled analysis of 575 cases. Epilepsia. 2015;56(11):1738-1746. doi:10.1111/epi.13128.

26. Hancock EC, Osborne JP, Edwards SW. Treatment of infantile spasms. Cochrane database Syst Rev. 2013;6:CD001770. doi:10.1002/14651858.CD001770.pub3.

27. Commins SP, Borish L, Steinke JW. Immunologic messenger molecules: Cytokines, interferons, and chemokines. J Allergy Clin Immunol. 2010;125(2):S53-S72. doi:10.1016/j.jaci.2009.07.008.

28. Williams JL, Holman DW, Klein RS. Chemokines in the balance: maintenance of homeostasis and protection at CNS barriers. Front Cell Neurosci. 2014;8:154. doi:10.3389/fncel.2014.00154.

29. Griffith JW, Sokol CL, Luster AD. Chemokines and chemokine receptors: positioning cells for host defense and immunity. Annu Rev Immunol. 2014;32:659-702. doi:10.1146/annurevimmunol-032713-120145.

30. Vezzani A, Ravizza T, Balosso S, Aronica E. Glia as a source of cytokines: implications for neuronal excitability and survival. Epilepsia. 2008;49 Suppl 2:24-32.

31. Boulanger LM. Immune Proteins in Brain Development and Synaptic Plasticity. Neuron. 2009;64(1):93109. doi:10.1016/j.neuron.2009.09.001.

32. Barres B a. The Mystery and Magic of Glia: A Perspective on Their Roles in Health and Disease. Neuron. 2008;60(3):430-440. doi:10.1016/j.neuron.2008.10.013.

33. Michell-Robinson MA, Touil $H$, Healy LM, et al. Roles of microglia in brain development, tissue maintenance and repair. Brain. 2015;138(5):1138-1159. doi:10.1093/brain/awv066.

34. Vezzani A, French J, Bartfai T, Baram TZ. The role of inflammation in epilepsy. Nat Rev Neurol. 2011;7(1):31-40. doi:10.2174/157339607782411538.

35. Vezzani A, Viviani B. Neuromodulatory properties of inflammatory cytokines and their impact on neuronal excitability. Neuropharmacology. 2015;96(Pt A):70-82. doi:10.1016/j. neuropharm.2014.10.027.

36. Wilcox KS, Vezzani A. Does brain inflammation mediate pathological outcomes in epilepsy? Adv Exp Med Biol. 2014;813:169-183. doi:10.1007/978-94-017-8914-1_14.37. _ A I a p i r t t i T, Rinta S, Hulkkonen J, Makinen R, Keranen T, Peltola J. Interleukin-6, interleukin-1 receptor antagonist and interleukin-1 beta production in patients with focal epilepsy: A video-EEG study. J Neurol Sci. 2009;280(1-2):94-97.

38. Bauer S, Cepok S, Todorova-Rudolph A, et al. Etiology and site of temporal lobe epilepsy influence postictal cytokine release. Epilepsy Res. 2009;86(1):82-88. doi:10.1016/j.eplepsyres.2009.05.009.

39. Uludag IF, Bilgin S, Zorlu Y, Tuna G, Kirkali G. Interleukin-6, interleukin-1 beta and interleukin-1 receptor antagonist levels in epileptic seizures. Seizure. 2013;22(6):457-461. doi:10.1016/j.seizure.2013.03.004.

40. Todd J, Simpson P, Estis J, Torres V, Wub AHB. Reference range and short- and long-term biological variation of interleukin (IL)-6, IL-17A and tissue necrosis factor-alpha using high sensitivity assays. Cytokine. 2013;64(3):660-665. doi:10.1016/j.cyto.2013.09.018.

41. Sheng JG, Boop FA, Mrak RE, Griffin WS. Increased neuronal beta-amyloid precursor protein expression in human temporal lobe epilepsy: association with interleukin-1 alpha immunoreactivity. J Neurochem. 1994;63(5):1872-1879.

42. Fiala M, Avagyan H, Merino JJ, et al. Chemotactic and mitogenic stimuli of neuronal apoptosis in patients with medically intractable temporal lobe epilepsy. Pathophysiology. 2013;20(1):59-69. doi:10.1016/j.pathophys.2012.02.003. 
Chapter 3. Inflammatory mediators in human epilepsy

43. Ravizza T, Gagliardi B, Noe F, Boer K, Aronica E, Vezzani A. Innate and adaptive immunity during epileptogenesis and spontaneous seizures: evidence from experimental models and human temporal lobe epilepsy. Neurobiol Dis. 2008;29(1):142-160. doi:S0969-9961(07)00193-3.

44. Omran A, Peng J, Zhang C, et al. Interleukin-1beta and microRNA-146a in an immature rat model and children with mesial temporal lobe epilepsy. Epilepsia. 2012;53(7):1215-1224. doi:10.1111 /j.1528-1167.2012.03540.

45. Lehtimaki KA, Keranen T, Palmio J, et al. Increased plasma levels of cytokines after seizures in localization-related epilepsy. Acta Neurol Scand. 2007;116(4):226-230.

46. Liimatainen S, Fallah M, Kharazmi E, Peltola M, Peltola J. Interleukin-6 levels are increased in temporal lobe epilepsy but not in extra-temporal lobe epilepsy. J Neurol. 2009;256(5):796-802. doi:10.1007/ s00415-009-5021-x.

47. Hulkkonen J, Koskikallio E, Rainesalo S, Keranen T, Hurme M, Peltola J. The balance of inhibitory and excitatory cytokines is differently regulated in vivo and in vitro among therapy resistant epilepsy patients. Epilepsy Res. 2004;59(2-3):199-205.

48. Nowak M, Bauer S, Haag A, et al. Interictal alterations of cytokines and leukocytes in patients with active epilepsy. Brain Behav Immun. 2011;25(3):423-428.

49. Lehtimäki KA, Liimatainen S, Peltola J, Arvio M. The serum level of interleukin-6 in patients with intellectual disability and refractory epilepsy. Epilepsy Res. 2011. doi:10.1016/j.eplepsyres.2011.03.004

50. Haspolat S, Mihci E, Coskun M, Gumuslu S, Ozben T, Yegin O. Interleukin-1beta, tumor necrosis factor-alpha, and nitrite levels in febrile seizures. J Child Neurol. 2002;17(10):749-751.

51. Tutuncuoglu S, Kutukculer N, Kepe L, Coker C, Berdeli A, Tekgul H. Proinflammatory cytokines, prostaglandins and zinc in febrile convulsions. Pediatr Int. 2001;43(3):235-239. doi:ped1389.

52. Choi J, Min HJ, Shin JS. Increased levels of HMGB1 and pro-inflammatory cytokines in children with febrile seizures. J Neuroinflammation. 2011;8:135. doi:10.1186/1742-2094-8-135.

53. Hu MH, Huang GS, Wu CT, et al. Analysis of plasma multiplex cytokines for children with febrile seizures and severe acute encephalitis. J Child Neurol. 2014;29(2):182-186.

54. Virta M, Hurme M, Helminen M. Increased plasma levels of pro- and anti-inflammatory cytokines in patients with febrile seizures. Epilepsia. 2002;43(8):920-923.

55. Ichiyama T, Suenaga N, Kajimoto M, et al. Serum and CSF levels of cytokines in acute encephalopathy following prolonged febrile seizures. Brain Dev. 2008;30(1):47-52. doi:S0387-7604(07)00143-X.

56. Okamoto R, Fujii S, Inoue T, et al. Biphasic clinical course and early white matter abnormalities may be indicators of neurological sequelae after status epilepticus in children. Neuropediatrics. 2006;37(1):32-41. doi:10.1055/s-2006-923949.

57. Fukumoto Y, Okumura A, Hayakawa F, et al. Serum levels of cytokines and EEG findings in children with influenza associated with mild neurological complications. Brain Dev. 2007;29(7):425-430.

58. Haginoya K, Noguchi R, Zhao Y, et al. Reduced levels of interleukin-1 receptor antagonist in the cerebrospinal fluid in patients with West syndrome. Epilepsy Res. 2009;85(2-3):314-317.

59. Shiihara T, Miyashita M, Yoshizumi M, Watanabe M, Yamada Y, Kato M. Peripheral lymphocyte subset and serum cytokine profiles of patients with West syndrome. Brain Dev. 2010;32(9):695-702. doi:10.1016/j.braindev.2009.11.001.

60. Lehtimaki KA, Liimatainen S, Peltola J, Arvio M. The serum level of interleukin-6 in patients with intellectual disability and refractory epilepsy. Epilepsy Res. 2011;95(1-2):184-187. doi:10.1016/j. eplepsyres.2011.03.004.

61. Shu HF, Zhang CQ, Yin Q, An N, Liu SY, Yang H. Expression of the interleukin 6 system in cortical lesions from patients with tuberous sclerosis complex and focal cortical dysplasia type Ilb. J Neuropathol Exp Neurol. 2010;69(8):838-849.

62. Pacifici R, Paris L, Carlo S Di, Bacosi A, Pichini S, Zuccaro P. Cytokine production in blood mononuclear cells from epileptic patients. Epilepsia. 1995;36(4):384-387. 
63. Helminen M, Vesikari T. Increased interleukin-1 (IL-1) production from LPS-stimulated peripheral blood monocytes in children with febrile convulsions. Acta Paediatr Scand. 1990;79(8-9):810-816.

64. Matsuo M, Sasaki K, Ichimaru T, Nakazato S, Hamasaki Y. Increased IL-1 beta production from dsRNAstimulated leukocytes in febrile seizures. Pediatr Neurol. 2006;35(2):102-106.

65. Nur BG, Sahinturk D, Coskun M, Duman O, Yavuzer U, Haspolat S. Single nucleotide polymorphism and production of IL-1 beta and IL-10 cytokines in febrile seizures. Neuropediatrics. 2012;43(4):194200. doi:10.1055/s-0032-1323849.

66. Straussberg R, Amir J, Harel L, Punsky I, Bessler H. Pro- and anti-inflammatory cytokines in children with febrile convulsions. Pediatr Neurol. 2001;24(1):49-53. doi:S0887-8994(00)00234-4.

67. Dundar NO, Aktekin B, Ekinci NC, et al. Interleukin-1 beta secretion in hippocampal sclerosis patients with mesial temporal lobe epilepsy. Neurol Int. 2013;5(3):e17. doi:10.4081/ni.2013.e17.

68. Lehtimäki K., Keränen T, Huhtala H, et al. Regulation of IL-6 system in cerebrospinal fluid and serum compartments by seizures: the effect of seizure type and duration. J Neuroimmunol. 2004;152(12):121-125. doi:10.1016/j.jneuroim.2004.01.024.

69. Hosnijeh FS, Krop EJM, Portengen L, et al. Stability and reproducibility of simultaneously detected plasma and serum cytokine levels in asymptomatic subjects. Biomarkers. 2010;15(September 2009):140-148. doi:10.3109/13547500903340570.

70. Coughlan CM, McManus CM, Sharron M, et al. Expression of multiple functional chemokine receptors and monocyte chemoattractant protein-1 in human neurons. Neuroscience. 2000;97(3):591-600.

71. Miller RJ, Rostene W, Apartis E, et al. Chemokine action in the nervous system. J Neurosci. 2008;28(46):11792-11795. doi:10.1523/JNEUROSCI.3588-08.2008.

72. Rostène $W$, Guyon $A$, Kular L, et al. Chemokines and chemokine receptors: new actors in neuroendocrine regulations. FrontNeuroendocrinol.2011;32(1):10-24. doi:10.1016/j.yfrne.2010.07.001.

73. Rostène W, Dansereau M-A, Godefroy D, et al. Neurochemokines: a menage a trois providing new insights on the functions of chemokines in the central nervous system. J Neurochem. 2011;118(5):680694. doi:10.1111/j.1471-4159.2011.07371.

74. Erickson MA, Dohi K, Banks WA. Neuroinflammation: a common pathway in CNS diseases as mediated at the blood-brain barrier. Neuroimmunomodulation. 2012;19(2):121-130. doi:10.1159/000330247.

75. Banks WA. The blood-brain barrier in neuroimmunology: Tales of separation and assimilation. Brain Behav Immun. August 2014. doi:10.1016/j.bbi.2014.08.007.

76. Chen $\mathrm{G}$, Reichlin S. Clearance of [1251]-tumor necrosis factor-alpha from the brain into the blood after intracerebroventricular injection in rats. Neuroimmunomodulation. 1998;5(5):261-269.

77. Romero LI, Kakucska I, Lechan RM, Reichlin S. Interleukin-6 (IL-6) is secreted from the brain after intracerebroventricular injection of IL-1 beta in rats. Am J Physiol. 1996;270(3 Pt 2):R518-24.

78. Friedman A, Heinemann U. Role of blood-brain barrier injury in epileptogenesis. Epilepsia. 2010;51(SUPPL. 5):34.

79. Huppert J, Closhen D, Croxford A, et al. Cellular mechanisms of IL-17-induced blood-brain barrier disruption. FASEB J. 2010;24(4):1023-1034. doi:10.1096/f.09-141978.

80. Waisman A, Hauptmann J, Regen T. The role of IL-17 in CNS diseases. Acta Neuropathol. 2015:625637. doi:10.1007/s00401-015-1402-7.

81. Kebir H, Kreymborg K, Ifergan I, et al. Human TH17 lymphocytes promote blood-brain barrier disruption and central nervous system inflammation. Nat Med. 2007;13(10):1173-1175. doi:10.1038/ nm1651.

82. Couper K, Blount D, Riley E. IL-10: the master regulator of immunity to infection. J Immunol. 2008;180(9):5771-5777. doi:10.4049/jimmunol.180.9.5771.

83. Pedersen BK, Steensberg a., Fischer C, Keller C, Ostrowski K, Schjerling P. Exercise and cytokines with particular focus on muscle-derived il-6. Exerc Immunol Rev. 2001;7(June):18-31.

84. Mehta V, Ferrie CD, Cross JH, Vadlamani G. Corticosteroids including ACTH for childhood epilepsy other than epileptic spasms. Cochrane database Syst Rev. 2015;6:CD005222. doi:10.1002/14651858. CD005222.pub3. 
Chapter 3. Inflammatory mediators in human epilepsy

85. Sinclair DB, Snyder TJ. Corticosteroids for the treatment of Landau-kleffner syndrome and continuous spike-wave discharge during sleep. Pediatr Neurol. 2005;32(5):300-306. doi:10.1016/j. pediatrneurol.2004.12.006.

86. Van Den Munckhof B, De Vries EE, Braun KPJ, et al. Serum inflammatory mediators correlate with disease activity in electrical status epilepticus in sleep (ESES) syndrome. Epilepsia. 2016;57(2):e45-e50. doi:10.1111/epi.13274.

87. Sharp M, Javidan M. Immunomodulation in adult epilepsy: the role of IVIG. Can J Neurol Sci. 2012;39:584-591.

88. van Rijckevorsel-Harmant K, Delire M, Schmitz-Moorman W, Wieser HG. Treatment of refractory epilepsy with intravenous immunoglobulins - Results of the first double-blind/dose finding clinical study. Int J Clin Lab Res. 1994;24:162-166. doi:10.1007/BF02592447.

89. Tracey KJ. Reflex control of immunity. Nat Pub/ Gr. 2009;9(6):418-428. doi:10.1038/nri2566.

90. Uthman BM, Reichl a M, Dean JC, et al. Effectiveness of vagus nerve stimulation in epilepsy patients: a 12-year observation. Neurology. 2004;63(6):1124-1126. doi:10.1212/01.WNL.0000138499.87068.C0.

91. Morris GL, MuellerWM. Long-term treatment with vagus nerve stimulation in patients with refractory epilepsy. The Vagus Nerve Stimulation Study Group E01-E05. Neurology. 1999;53(8):1731-1735.

92. Englot DJ, Chang EF, Auguste KI. Vagus nerve stimulation for epilepsy: a meta-analysis of efficacy and predictors of response. J Neurosurg. 2011;115(6):1248-1255. doi:10.3171/2011.7.JNS11977.

93. Dupuis N, Curatolo N, Benoist J-F, Auvin S. Ketogenic diet exhibits anti-inflammatory properties. Epilepsia. 2015;56(7):e95-8. doi:10.1111/epi.13038.

94. Klein P, Tyrlikova I, Mathews GC. Dietary treatment in adults with refractory epilepsy: A review. Neurology. 2014;83(21):1978-1985. doi:10.1212/WNL.0000000000001004.

95. Abou-Khalil B, Andermann E, Andermann F, Olivier A, Quesney LF. Temporal lobe epilepsy after prolonged febrile convulsions: excellent outcome after surgical treatment. Epilepsia. 1993;34(5):878883.

96. Cendes F, Andermann F, Dubeau F, et al. Early childhood prolonged febrile convulsions, atrophy and sclerosis of mesial structures, and temporal lobe epilepsy: an MRI volumetric study. Neurology. 1993:43(6):1083-1087.

97. Fukuda M, Hino H, Suzuki Y, Takahashi H, Morimoto T, Ishii E. Postnatal interleukin-1 beta enhances adulthood seizure susceptibility and neuronal cell death after prolonged experimental febrile seizures in infantile rats. Acta Neurol Belg. September 2013. doi:10.1007/s13760-013-0246-y.

98. Dubé CM, Ravizza T, Hamamura M, et al. Epileptogenesis provoked by prolonged experimental febrile seizures: mechanisms and biomarkers. J Neurosci. 2010;30(22):7484-7494. doi:10.1523/ JNEUROSCI.0551-10.2010.

99. Vezzani A, Moneta D, Conti M, et al. Powerful anticonvulsant action of IL-1 receptor antagonist on intracerebral injection and astrocytic overexpression in mice. Proc Natl Acad Sci U S A. 2000;97(21):11534-11539.

100. Vezzani A, Moneta D, Richichi C, et al. Functional role of inflammatory cytokines and antiinflammatory molecules in seizures and epileptogenesis. Epilepsia. 2002;43 Suppl 5:30-35. doi:epis6012.

101. Kalueff A V, Lehtimaki KA, Ylinen A, Honkaniemi J, Peltola J. Intranasal administration of human IL-6 increases the severity of chemically induced seizures in rats. Neurosci Lett. 2004;365(2):106-110. doi:10.1016/j.neulet.2004.04.061.

102. Sayyah M, Javad-Pour M, Ghazi-Khansari M. The bacterial endotoxin lipopolysaccharide enhances seizure susceptibility in mice: involvement of proinflammatory factors: nitric oxide and prostaglandins. Neuroscience. 2003;122(4):1073-1080.

103. Auvin S, Shin D, Mazarati A, Sankar R. Inflammation induced by LPS enhances epileptogenesis in immature rat and may be partially reversed by IL1RA. Epilepsia. 2010;51 Suppl 3:34-38. doi:10.1111/ j.1528-1167.2010.02606.x. 
104. Marchi N, Fan Q, Ghosh C, et al. Antagonism of peripheral inflammation reduces the severity of status epilepticus. Neurobiol Dis. 2009;33(2):171-181.

105. Willrich M a. V, Murray DL, Snyder MR. Tumor necrosis factor inhibitors: clinical utility in autoimmune diseases. Transl Res. 2015;165(2):270-282. doi:10.1016/j.trsl.2014.09.006.

106. Amedei A, Prisco D, D'Elios MM. Multiple sclerosis: The role of cytokines in pathogenesis and in therapies. Int J Mol Sci. 2012;13:13438-13460. doi:10.3390/ijms131013438.

107. Vezzani A, Moneta $\mathrm{D}$, Conti $\mathrm{M}$, et al. Powerful anticonvulsant action of IL-1 receptor antagonist on intracerebral injection and astrocytic overexpression in mice. Proc Natl Acad Sci U S A. 2000;97(21):11534-11539. doi:10.1073/pnas.190206797.

108. Librizzi L, Noe F, Vezzani A, de Curtis M, Ravizza T. Seizure-induced brain-borne inflammation sustains seizure recurrence and blood-brain barrier damage. Ann Neurol. 2012;72(1):82-90.

109. Bar-Klein G, Cacheaux LP, Kamintsky L, et al. Losartan prevents acquired epilepsy via TGF-beta signaling suppression. Ann Neurol. 2014. doi:10.1002/ana.24147.

110. Tchekalarova JD, Ivanova NM, Pechlivanova DM, et al. Pharmacology, Biochemistry and Behavior Antiepileptogenic and neuroprotective effects of losartan in kainate model of temporal lobe epilepsy. Pharmacol Biochem Behav. 2014;127:27-36. doi:10.1016/j.pbb.2014.10.005.

111. Maroso M, Balosso S, Ravizza T, et al. Toll-like receptor 4 and high-mobility group box-1 are involved in ictogenesis and can be targeted to reduce seizures. Nat Med. 2010;16(4):413-419. doi:10.1038/ nm.2127.

112. Cardoso AL, Guedes JR, Pereira de Almeida L, Pedroso de Lima MC. miR-155 modulates microgliamediated immune response by down-regulating SOCS-1 and promoting cytokine and nitric oxide production. Immunology. 2012;135(1):73-88. doi:10.1111/j.1365-2567.2011.03514.

113. Kan A a., Van Erp S, Derijck A a H a, et al. Genome-wide microRNA profiling of human temporal lobe epilepsy identifies modulators of the immune response. Cell Mol Life Sci. 2012;69(18):3127-3145. doi:10.1007/s00018-012-0992-7.

114. Wannamaker W, Davies R, Namchuk M, et al. (S)-1-((S)-2-\{[1-(4-Amino-3-chloro-phenyl)-methanoyl]amino\}-3,3-dimethyl-butanoyl)-pyrrolidine-2-carboxylic acid ((2R,3S)-2-ethoxy-5-oxo-tetrahydrofuran-3-yl)-amide (VX-765), an Orally Available Selective Interleukin (IL)-Converting Enzyme/ Caspase-1 Inh. J Pharmacol Exp Ther. 2007;321(2):509-516. doi:10.1124/jpet.106.111344.

115. French J, Chen Y, Fan X, et al. VX-765, a novel, investigational anti-inflammatory agent which inhibits IL-1 $\beta$ production: Proof-of-concept trial for refractory partial onset seizures. Epilepsy Curr. 2012;12(1 (SUPPL.1)):275.

116. French J, Sperling M, Perucca E, et al. A randomized, double-blind, placebocontrolled study to evaluate the efficacy, safety and tolerability of VX-765 in patients with treatment-resistant focal seizures. Epilepsy Curr. 2014;14(SUPPL 1):440-441.

117. Walker L, Sills GJ. Inflammation and epilepsy: The foundations for a new therapeutic approach in epilepsy? Epilepsy Curr. 2012;12(11):8-12. doi:10.5698/1535-7511-12.1.8.

118. Bartel DP. MicroRNAs: genomics, biogenesis, mechanism, and function. Cell. 2004;116(2):281-297. doi:10.1016/S0092-8674(04)00045-5.

119. Ponomarev ED, Veremeyko T, Weiner HL. MicroRNAs are universal regulators of differentiation, activation, and polarization of microglia and macrophages in normal and diseased CNS. Glia. 2013;61(1):91-103. doi:10.1002/glia.22363. 


\section{Supplementary Material}

\section{Supplementary material 1 - all individual meta-analyses \\ General remark}

This appendix contains all meta-analyses that were used to build the overview in figures 3A and 3B. Abbreviations: FS: febrile seizures, NS: neonatal seizures, TLE: temporal lobe epilepsy, PTE: post-traumatic epilepsy, unspecified: see appendix 2, WS: West syndrome.

\section{Serum}

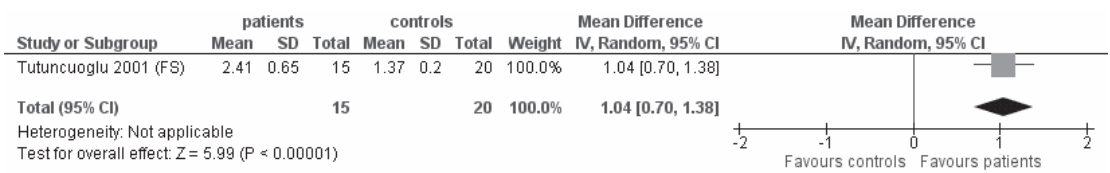

Meta-analysis 1 . Serum IL-1a

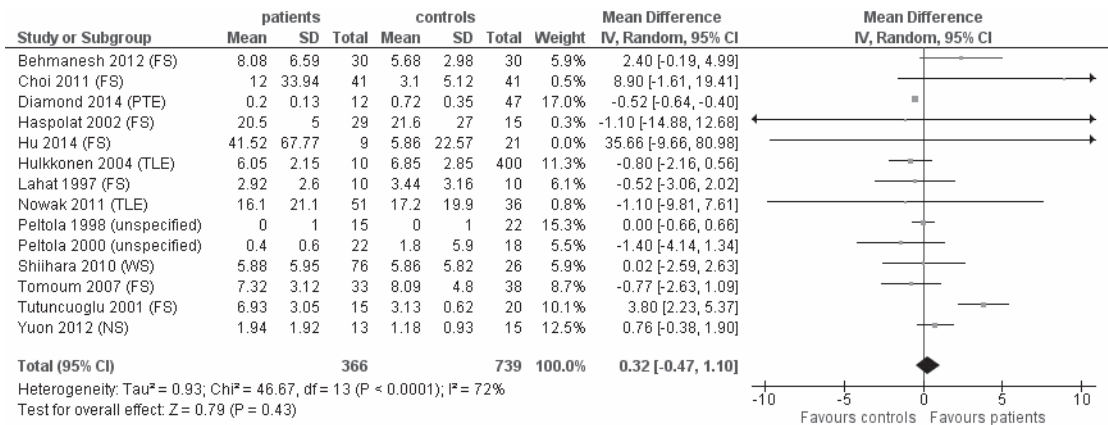

Meta-analysis 2 . Serum IL-1 $\beta$

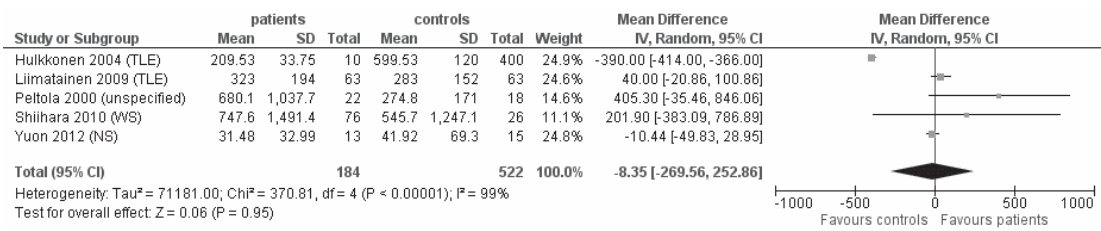

Meta-analysis 3. Serum IL-1ra 


\begin{tabular}{|c|c|c|c|c|c|c|c|c|c|c|}
\hline \multirow[b]{2}{*}{ Study or Subgroup } & \multicolumn{2}{|c|}{ patients } & \multicolumn{4}{|c|}{ controls } & \multicolumn{2}{|r|}{ Mean Difference } & \multirow{2}{*}{\multicolumn{2}{|c|}{$\begin{array}{c}\text { Mean Difference } \\
\text { IV, Random, } 95 \% \mathrm{Cl}\end{array}$}} \\
\hline & Mean & SD & Total & Mean & SD & Total & Weight & N, Random, $95 \% \mathrm{Cl}$ & & \\
\hline Hu 2014 (FS) & 486.38 & 809.12 & 9 & 129.36 & 212.25 & 20 & $0.0 \%$ & $357.02[-179.72,893.76]$ & & \\
\hline Liu 2001 (WS) & 0.37 & 0.12 & 10 & 0.24 & 0.07 & 15 & $84.3 \%$ & $0.13[0.05,0.21]$ & & - \\
\hline Shiihara 2010 (WS) & 18.78 & 35.99 & 76 & 12.14 & 39.76 & 26 & $0.1 \%$ & $6.64[-10.65,23.93]$ & & \\
\hline Yuon 2012 (NS) & 1.94 & 2.09 & 13 & 1.11 & 1.02 & 15 & $15.6 \%$ & $0.83[-0.42,2.08]$ & & $\longrightarrow$ \\
\hline Total $(95 \% \mathrm{Cl})$ & & & 108 & & & 76 & $100.0 \%$ & $0.25[-0.30,0.79]$ & & \\
\hline $\begin{array}{l}\text { Heterogeneity: } \mathrm{Tau}^{2}= \\
\text { Test for overall effect }\end{array}$ & $\begin{array}{l}0.09 ; \mathrm{Chi}^{-} \\
\mathrm{z}=0.88\end{array}$ & $\begin{array}{l}\mathrm{i}^{2}=3.45 \\
(\mathrm{P}=0.38)\end{array}$ & $d f=3(P$ & $P=0.33) ;$ & $I^{2}=13 \%$ & & & & $\begin{array}{c} \\
-0.5 \\
\text { Favours controls }\end{array}$ & $\begin{array}{lc}0 & 0.5 \\
\text { Favours patients }\end{array}$ \\
\hline
\end{tabular}

\section{Meta-analysis 4. Serum IL-2}

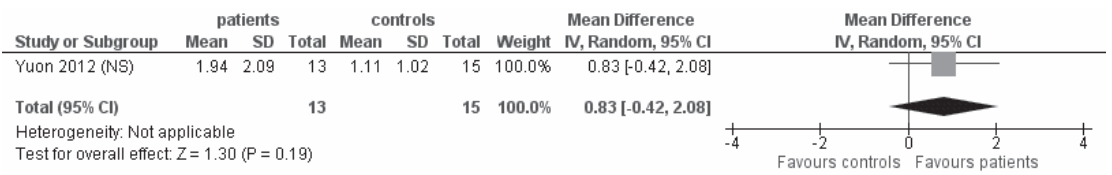

Meta-analysis 5. Serum IL-3

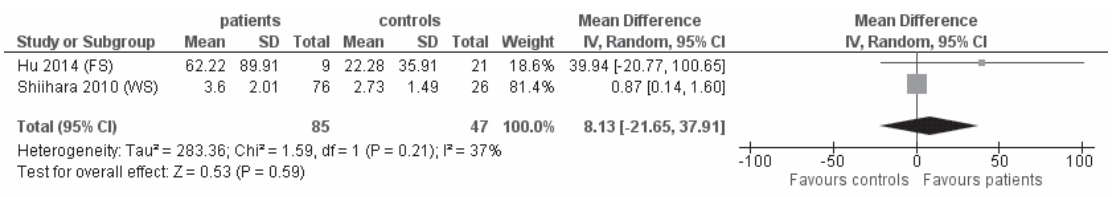

\section{Meta-analysis 6. Serum IL-4}

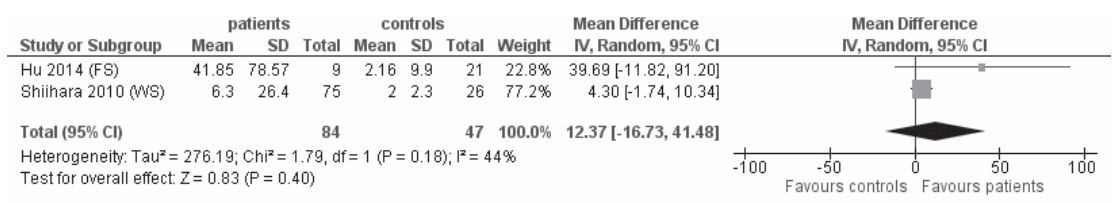

Meta-analysis 7. Serum IL-5

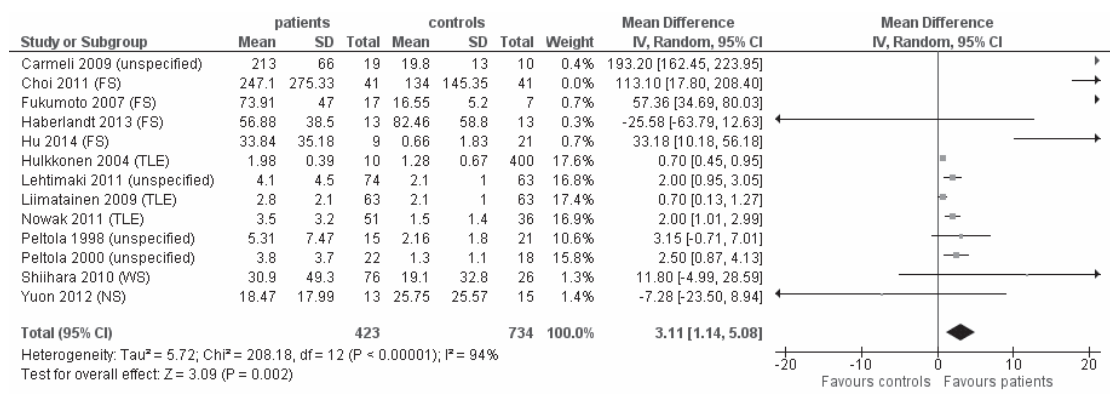

\section{Meta-analysis 8. Serum IL-6}




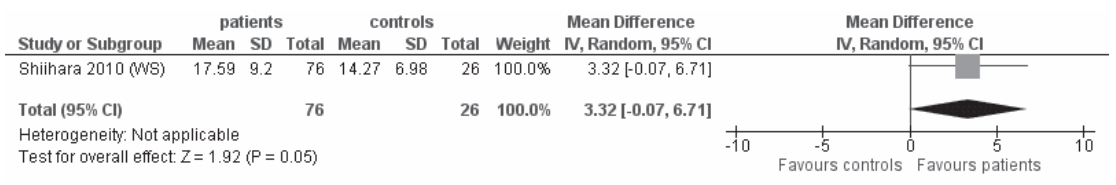

Meta-analysis 9. Serum IL-7

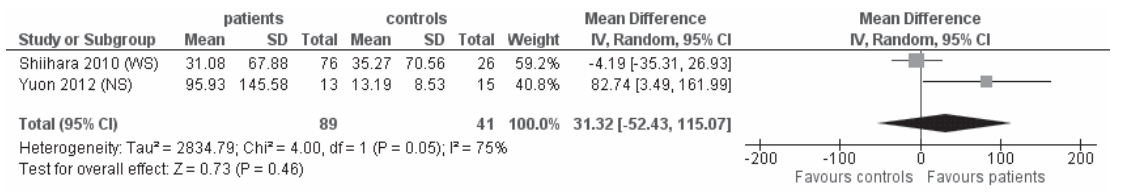

\section{Meta-analysis 10 . Serum IL-8}

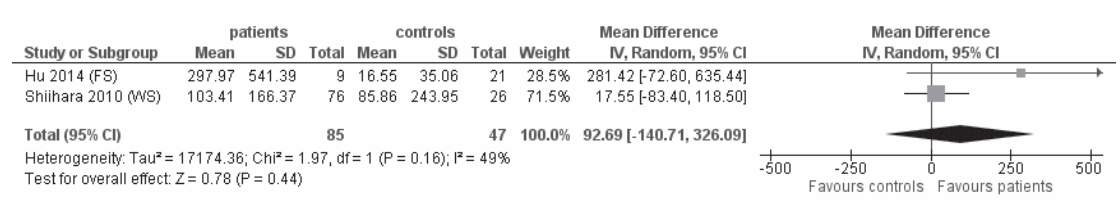

Meta-analysis 11. Serum IL-9

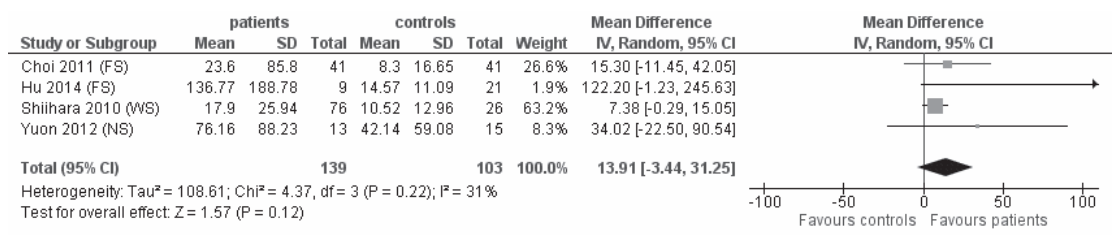

Meta-analysis 12. Serum IL-10

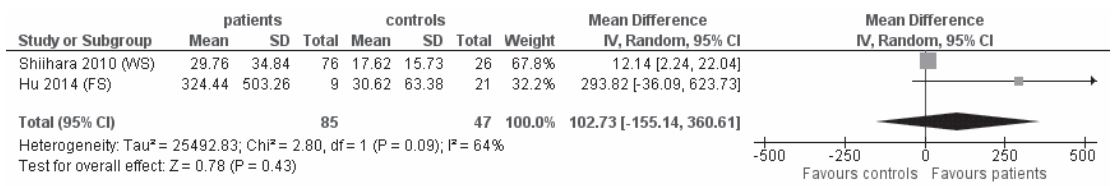

Meta-analysis 13. Serum IL-12

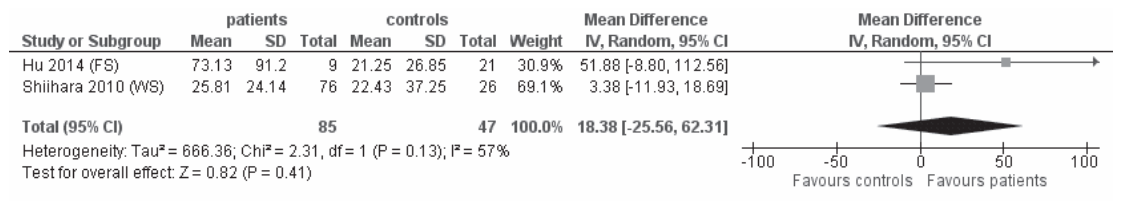

Meta-analysis 14. Serum IL-13 


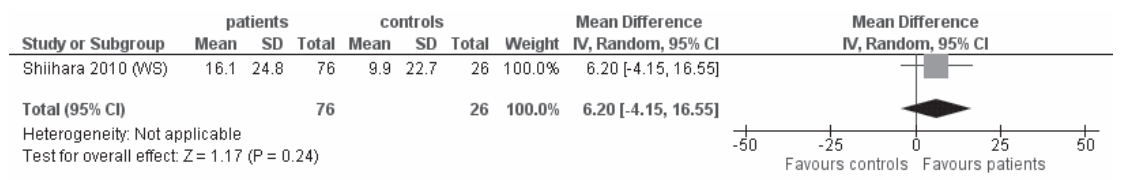

\section{Meta-analysis 15 . Serum IL-15}

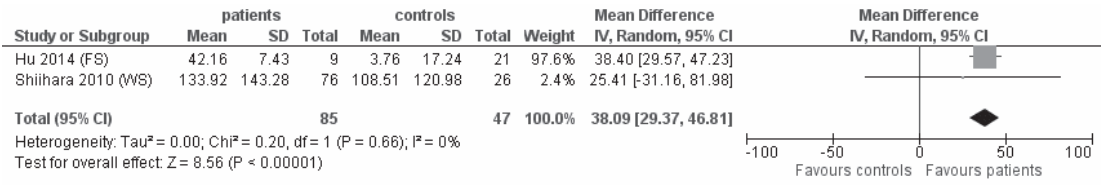

\section{Meta-analysis 16. Serum IL-17}

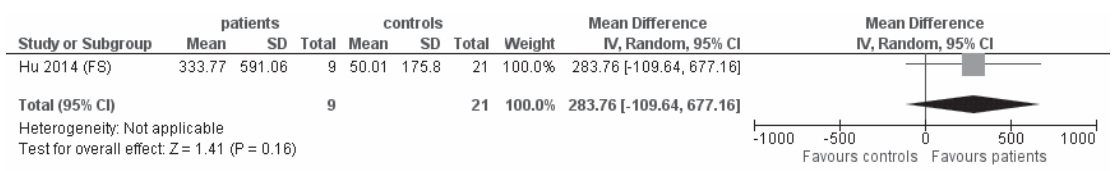

\section{Meta-analysis 17. Serum IL-22}

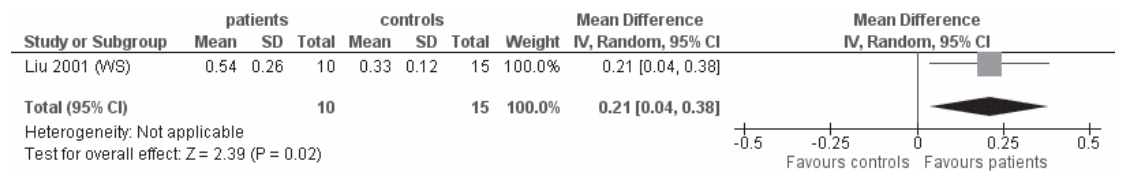

\section{Meta-analysis 18 . Serum IFN-a}

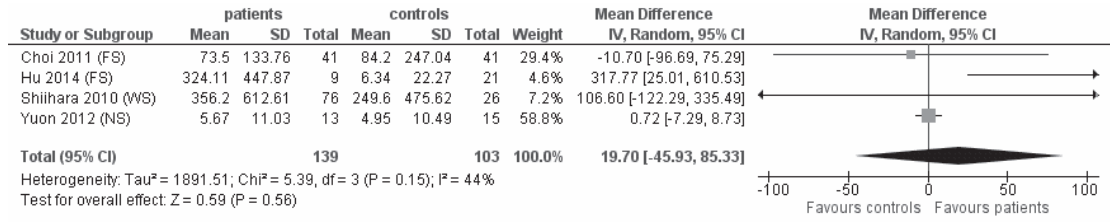

\section{Meta-analysis 19. Serum IFN- $\gamma$}

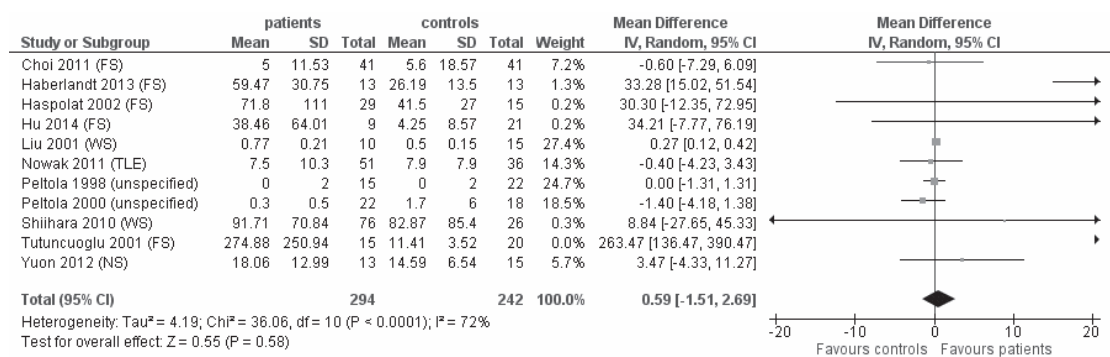




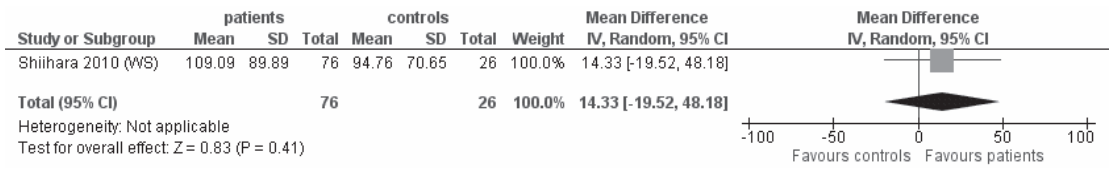

Meta-analysis 21. Serum CCL2

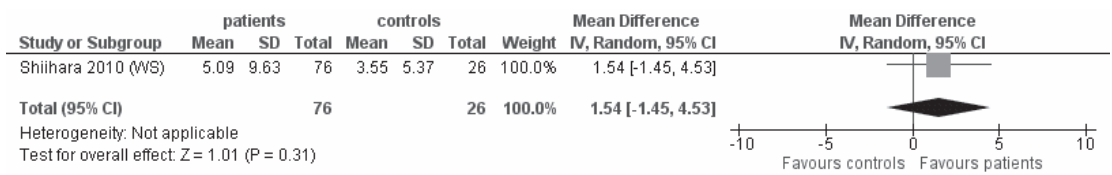

Meta-analysis 22. Serum CCL3

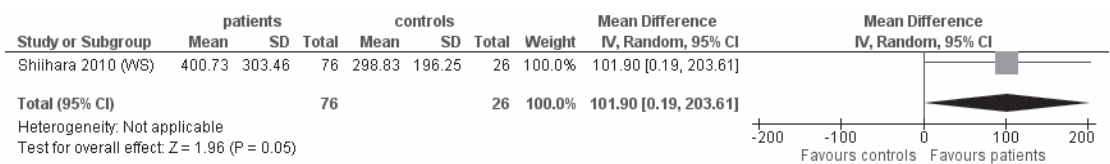

\section{Meta-analysis 23. Serum CCL4}

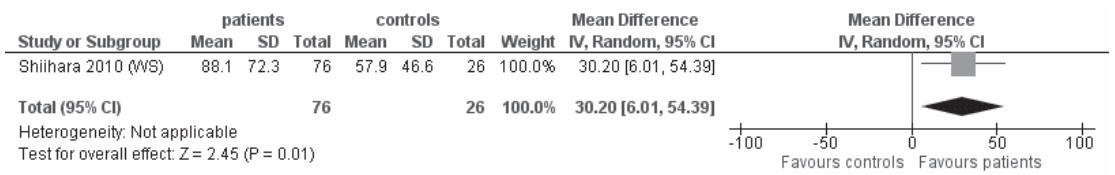

\section{Meta-analysis 24. Serum CCL11}

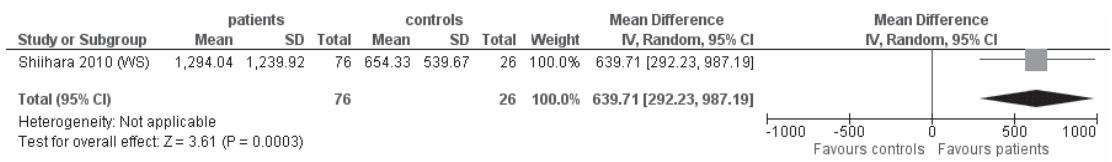

\section{Meta-analysis 25. Serum CXCL10}

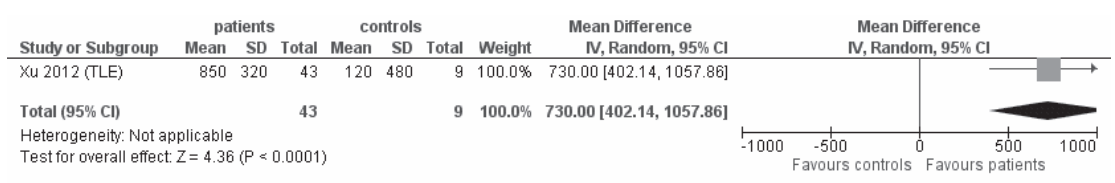

Meta-analysis 26. Serum CX3CL1 


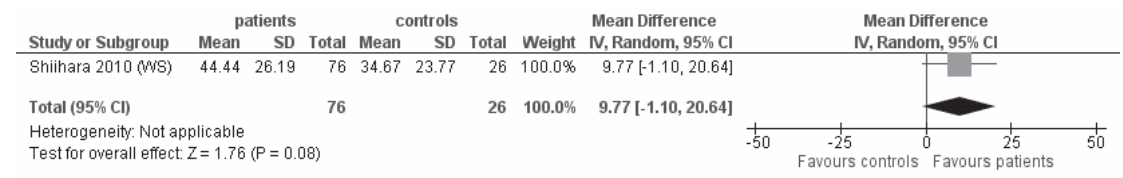

\section{Meta-analysis 27. Serum G-CSF}

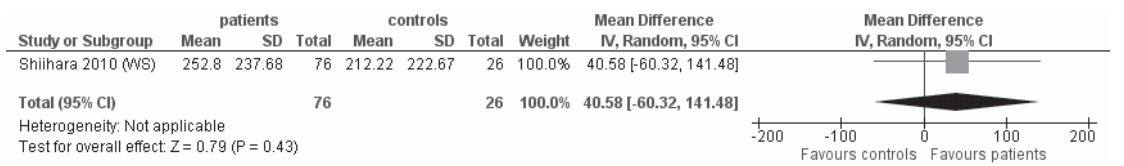

\section{Meta-analysis 28. Serum GM-CSF}

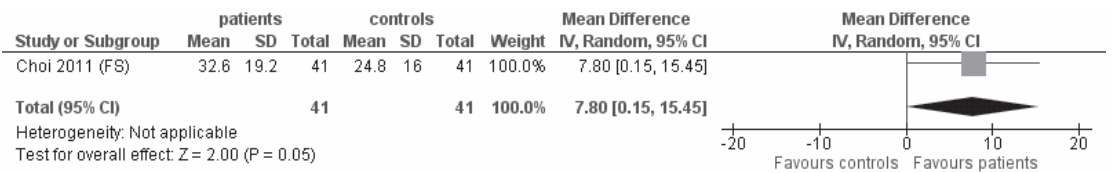

Meta-analysis 29. Serum HMGB1

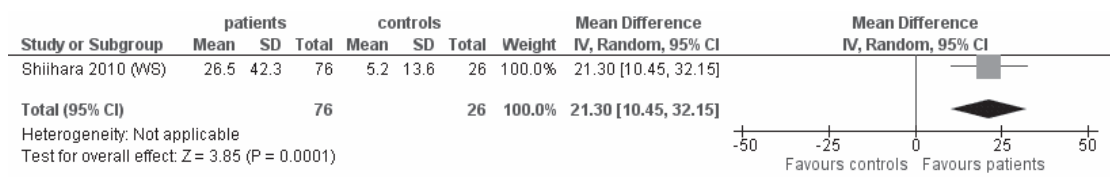

\section{Meta-analysis 30. Serum bFGF}

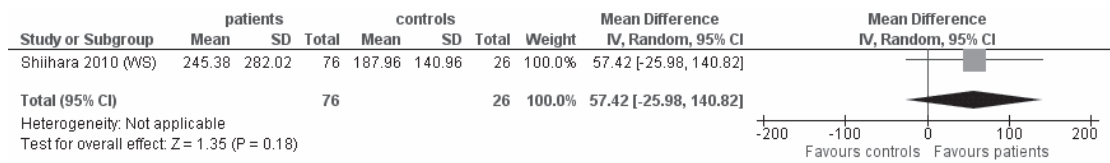

\section{Meta-analysis 31. Serum VEGF}

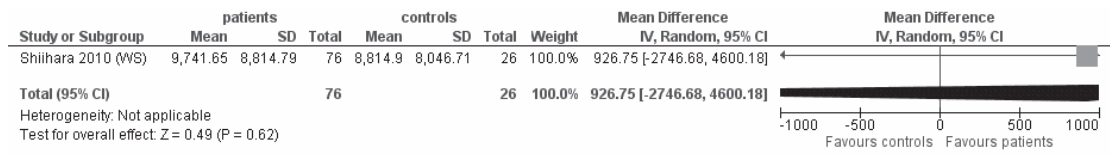

\section{Meta-analysis 32. Serum PDGF $\beta$}




\section{Cerebrospinal fluid (CSF)}

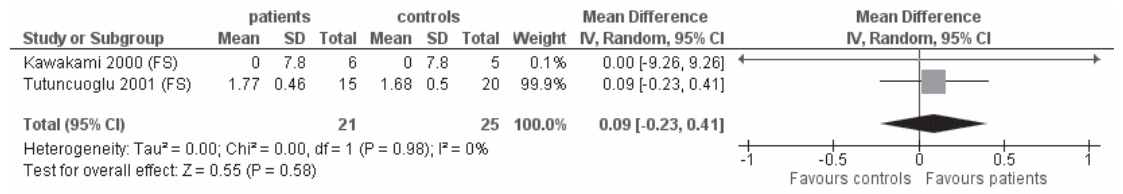

Meta-analysis 33. CSF IL-1a

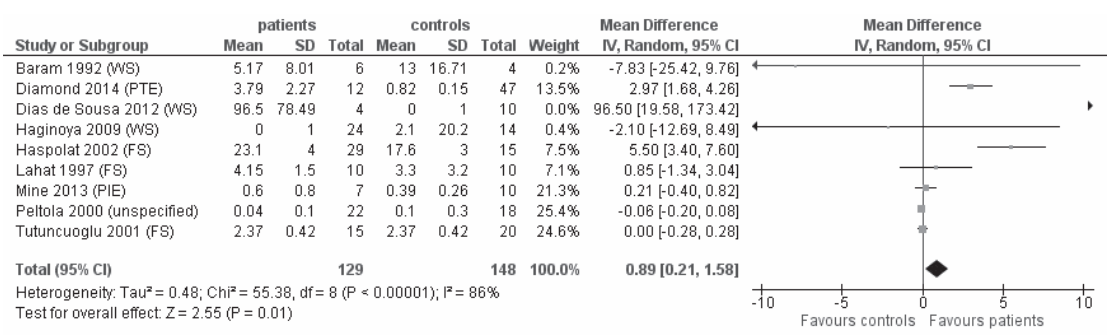

\section{Meta-analysis 34. CSF IL-1 $\beta$}

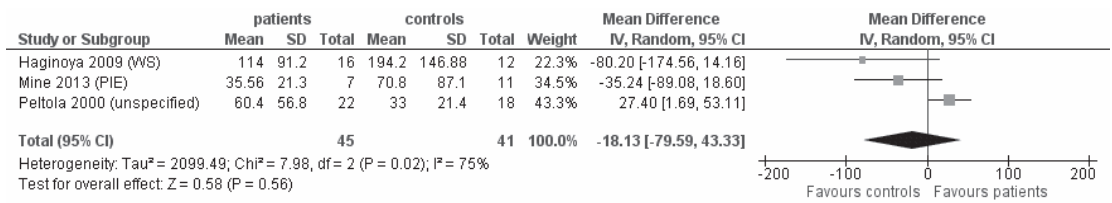

\section{Meta-analysis 35. CSF IL-1 ra}

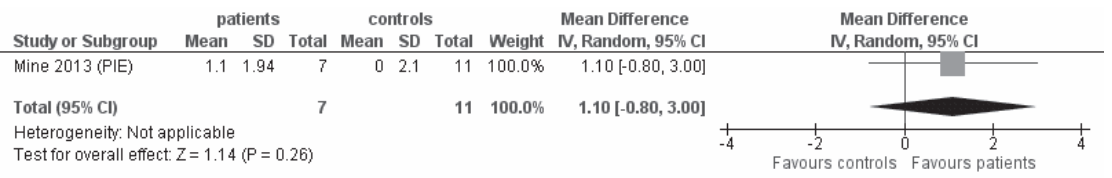

Meta-analysis 36. CSF IL-2

\begin{tabular}{|c|c|c|c|c|c|c|c|c|c|c|}
\hline \multirow[b]{2}{*}{ Study or Subgroup } & \multicolumn{3}{|c|}{ patients } & \multicolumn{3}{|c|}{ controls } & \multicolumn{2}{|r|}{ Mean Difference } & \multirow{2}{*}{\multicolumn{2}{|c|}{$\begin{array}{c}\text { Mean Difference } \\
\text { IV, Random, } 95 \% \mathrm{Cl}\end{array}$}} \\
\hline & Mean & SD & Total & Mean & SD & Total & Weight & IV, Random, $95 \% \mathrm{Cl}$ & & \\
\hline Mine 2013 (PIE) & 0.48 & 0.61 & 7 & 0.16 & 0.14 & 11 & $100.0 \%$ & $0.32[-0.14,0.78]$ & & \\
\hline Total $(95 \% \mathrm{Cl})$ & & & 7 & & & 11 & $100.0 \%$ & $0.32[-0.14,0.78]$ & & \\
\hline $\begin{array}{l}\text { Heterogeneity: Not a } \\
\text { Test for overall effec }\end{array}$ & $\begin{array}{l}\text { plicable } \\
Z=1.37\end{array}$ & & & & & & & & $\begin{array}{cc}-1 & 0 \\
\text { Favours controls } & 0 \\
\text { Favours pe }\end{array}$ & $\begin{array}{l}1 \\
\text { patients }\end{array}$ \\
\hline
\end{tabular}

Meta-analysis 37. CSF IL-4 


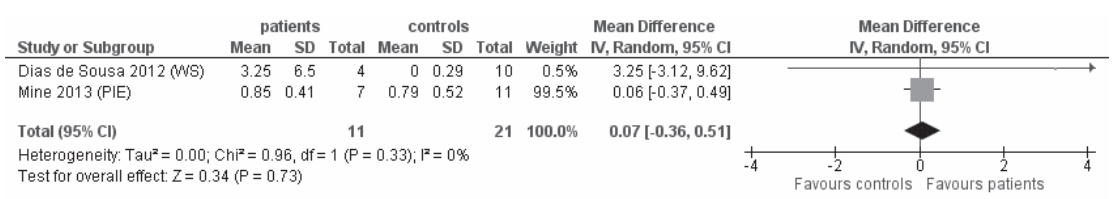

Meta-analysis 38. CSF IL-5

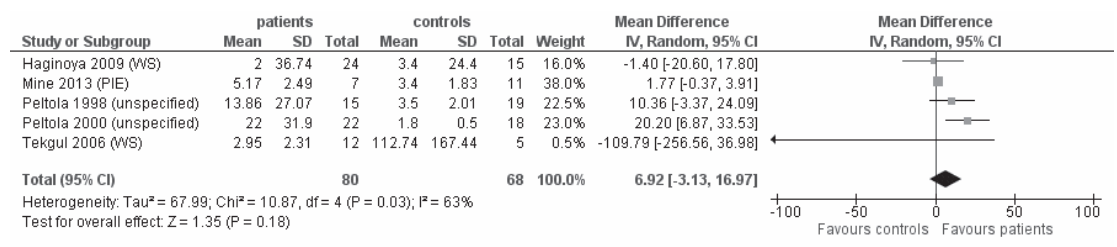

\section{Meta-analysis 39. CSF IL-6}

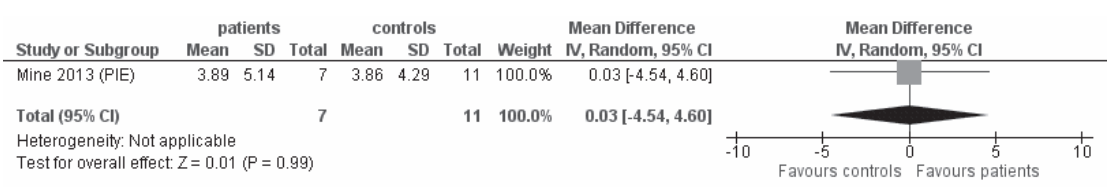

Meta-analysis 40. CSF IL-7

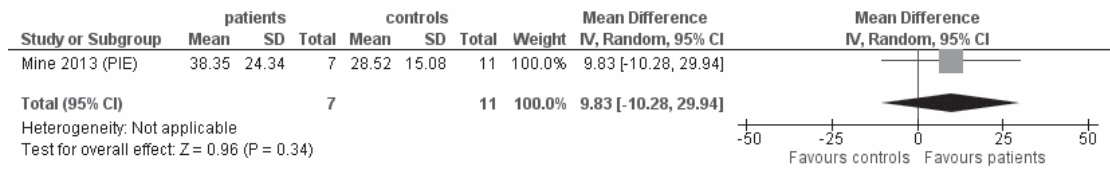

Meta-analysis 41. CSF IL-8

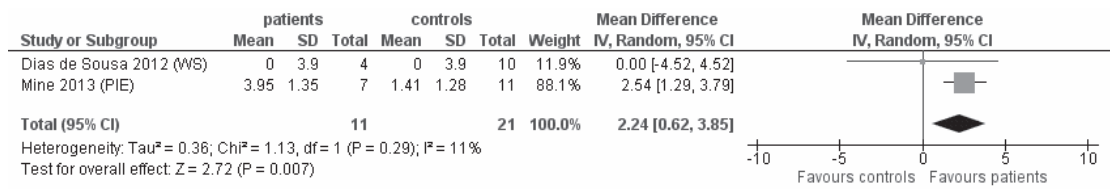

Meta-analysis 42. CSF IL-10

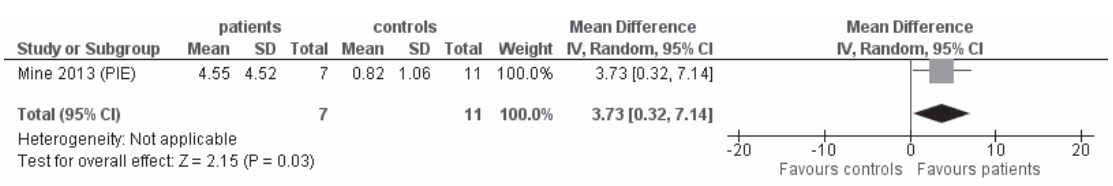

Meta-analysis 43. CSF IL-12 


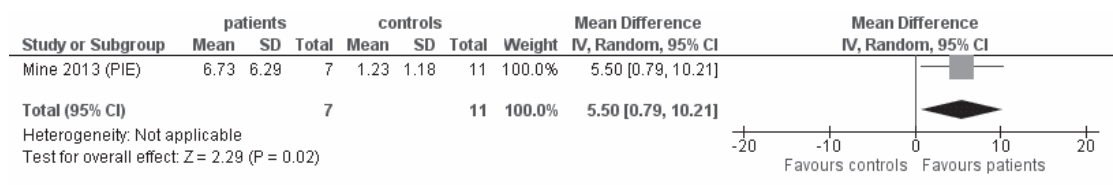

Meta-analysis 44. CSF IL-13

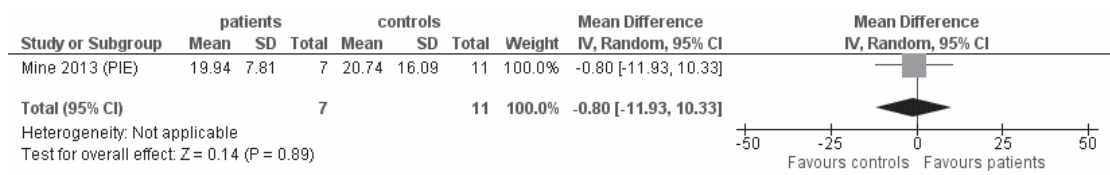

Meta-analysis 45. CSF IL-15

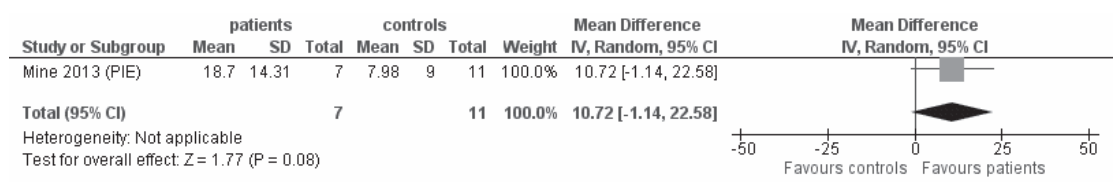

Meta-analysis 46. CSF IL-17

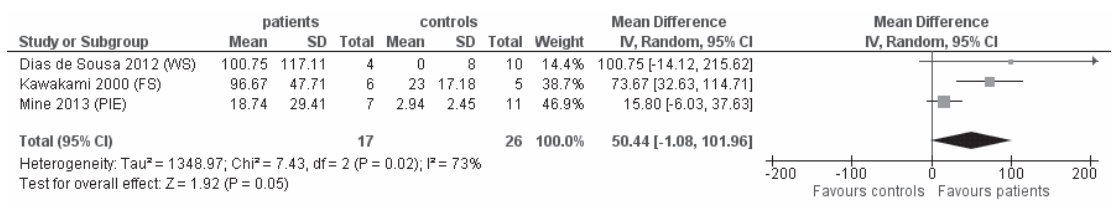

Meta-analysis 47. CSF IFN- $\gamma$

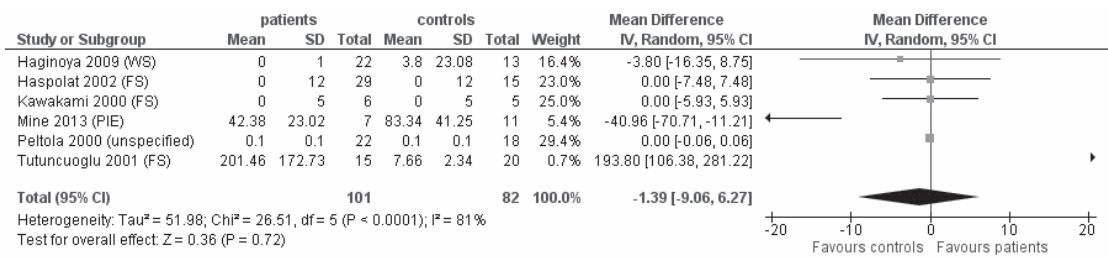

Meta-analysis 48. CSF TNF-a

\begin{tabular}{|c|c|c|c|c|c|c|c|c|c|c|c|c|}
\hline \multirow[b]{2}{*}{ Study or Subgroup } & \multicolumn{3}{|c|}{ patients } & \multicolumn{3}{|c|}{ controls } & \multirow[b]{2}{*}{ Weight } & \multicolumn{2}{|l|}{ Mean Difference } & \multirow{2}{*}{\multicolumn{2}{|c|}{$\begin{array}{c}\text { Mean Difference } \\
\text { I, Random, } 95 \% \mathrm{CI}\end{array}$}} & \\
\hline & Mean & SD & Total & Mean & SD & Total & & IV, Random, $95 \% \mathrm{Cl}$ & & & & \\
\hline XU 2012 (TLE) & 230 & 120 & 39 & 9 & 7 & 8 & $100.0 \%$ & $221.00[183.03,258.97]$ & & & & \\
\hline Total $(95 \% \mathrm{Cl})$ & & & 39 & & & 8 & $100.0 \%$ & $221.00[183.03,258.97]$ & & & & \\
\hline $\begin{array}{l}\text { Heterogeneity: Not a } \\
\text { Test for overall effect }\end{array}$ & $\begin{array}{l}\text { plicable } \\
Z=11.4\end{array}$ & & & & & & & & $\frac{+}{-500}$ & $\begin{array}{c}-250 \\
\text { Favours controls }\end{array}$ & Favours p & $\frac{500}{50}$ \\
\hline
\end{tabular}

Meta-analysis 49. CSF CX3CL1 


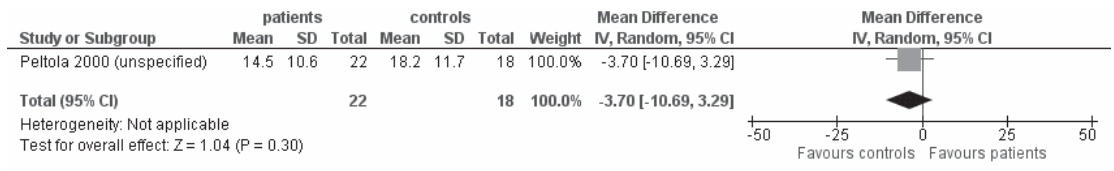

Meta-analysis 50. CSF NGF

\section{Supplementary material 2 - meta-analyses grouped by etiology}

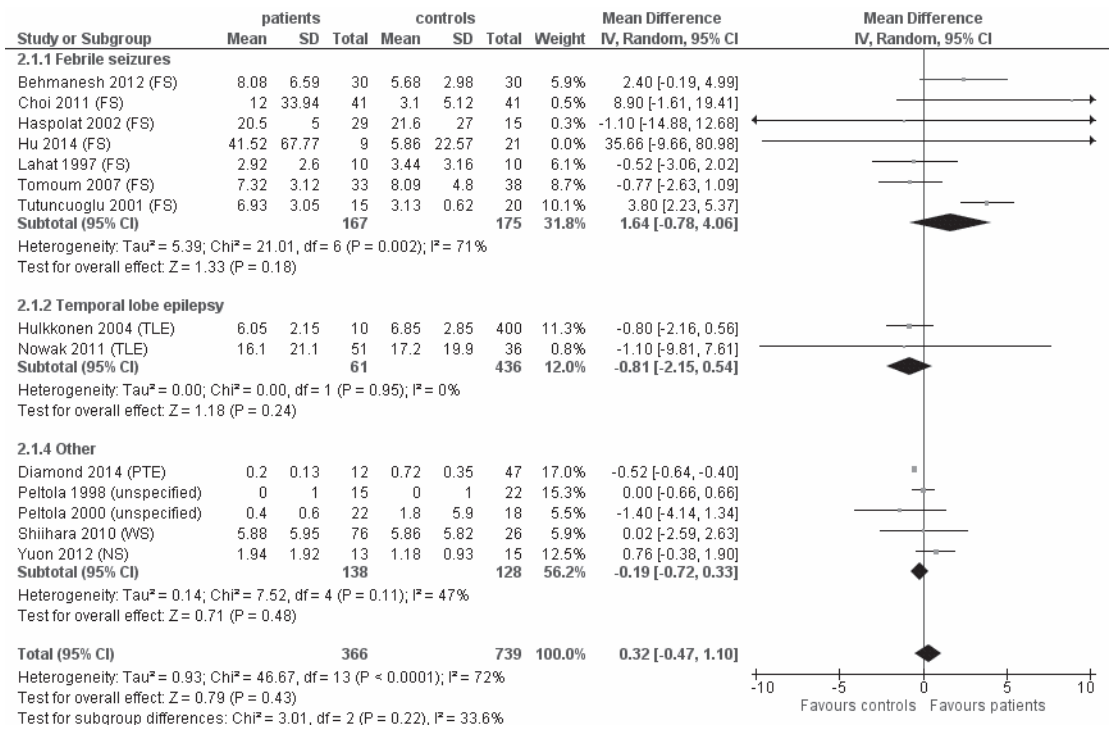

Meta-analysis 1 . Serum IL-1 $\beta$ concentration in epilepsy patients of various etiologies (14 studies). Analysis grouped by etiology.

FS: febrile seizures, NS: neonatal seizures, TLE: temporal lobe epilepsy, PTE: post-traumatic epilepsy, unspecified: see appendix 2, WS: West syndrome. 


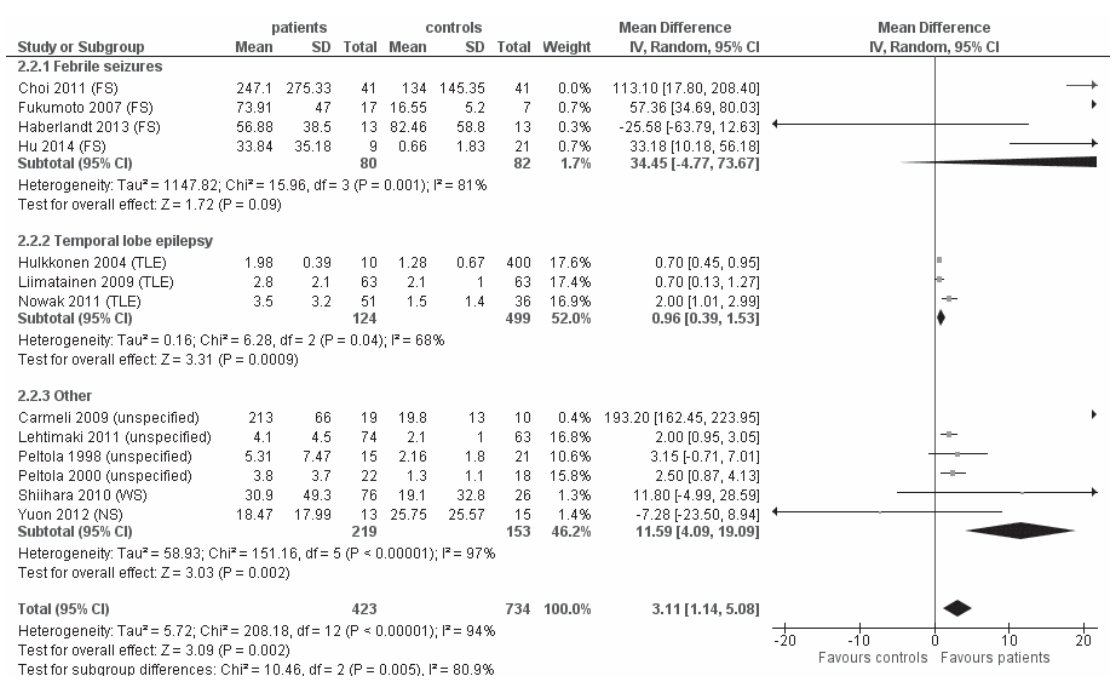

Meta-analysis 2. Serum IL-6 concentration in epilepsy patients of various etiologies (13 studies). Analysis grouped by etiology.

FS: febrile seizures, NS: neonatal seizures, TLE: temporal lobe epilepsy, unspecified: see appendix 2, WS: West syndrome.

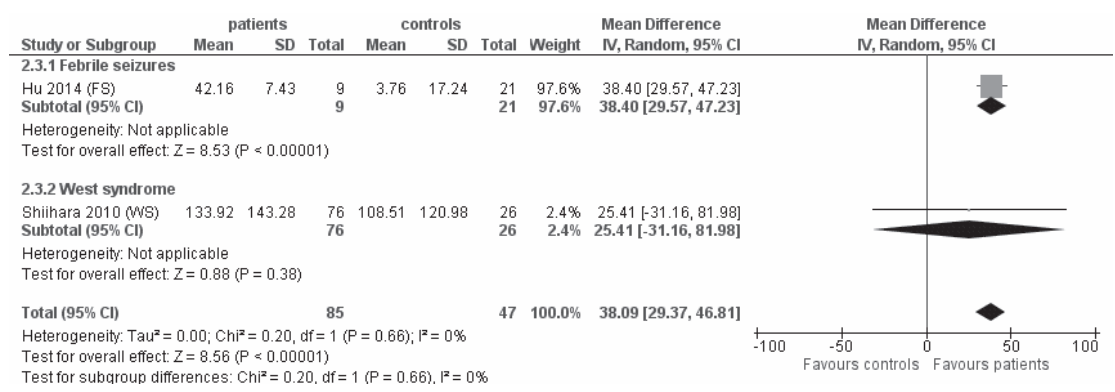

Meta-analysis 3. Serum IL-17 concentration in epilepsy patients of various etiologies (2 studies). Analysis grouped by etiology.

FS: febrile seizures, WS: West syndrome. 


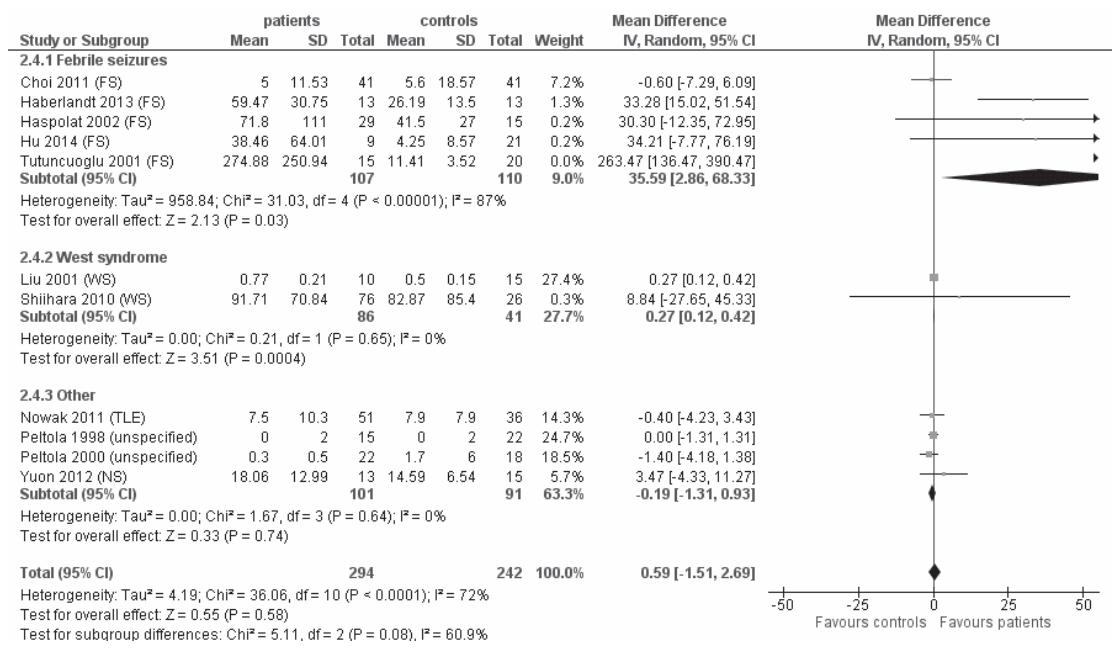

Meta-analysis 4. Serum TNF-a concentration in epilepsy patients of various etiologies (11 studies). Analysis grouped by etiology.

FS: febrile seizures, NS: neonatal seizures, TLE: temporal lobe epilepsy, unspecified: see appendix 2, WS: West syndrome.

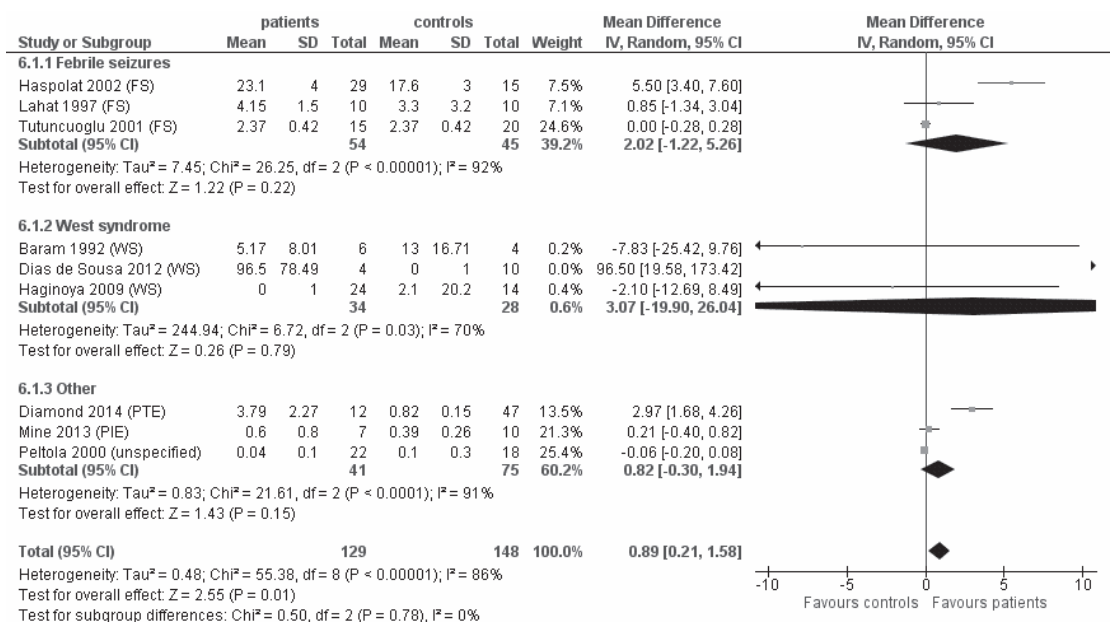

Meta-analysis 5. CSF IL-1 $\beta$ concentration in epilepsy patients of various etiologies (9 studies). Analysis grouped by etiology.

FS: febrile seizures, PIE: post-infectious epilepsy, PTE: post-traumatic epilepsy, unspecified: see appendix 2, WS: West syndrome. 


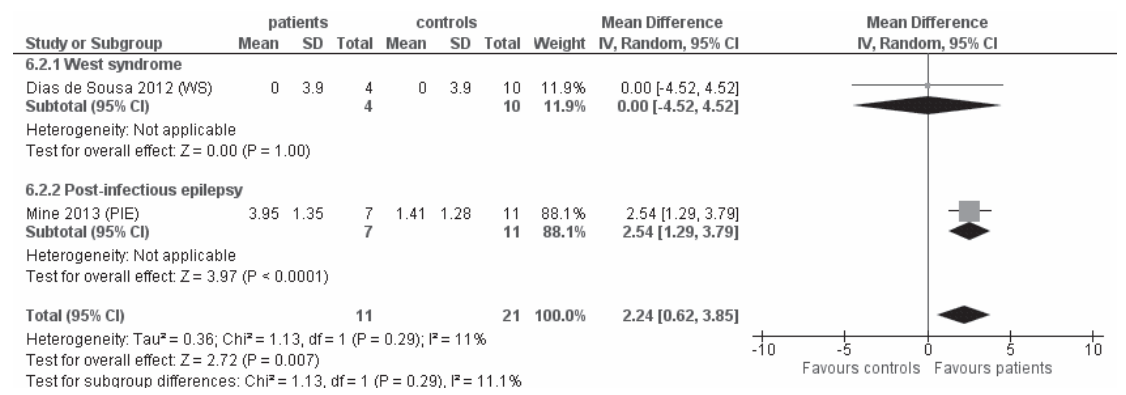

Meta-analysis 6. CSF IL-10 concentration in epilepsy patients of various etiologies ( 2 studies). Analysis grouped by etiology.

PIE: post-infectious epilepsy, WS: West syndrome. 


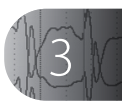



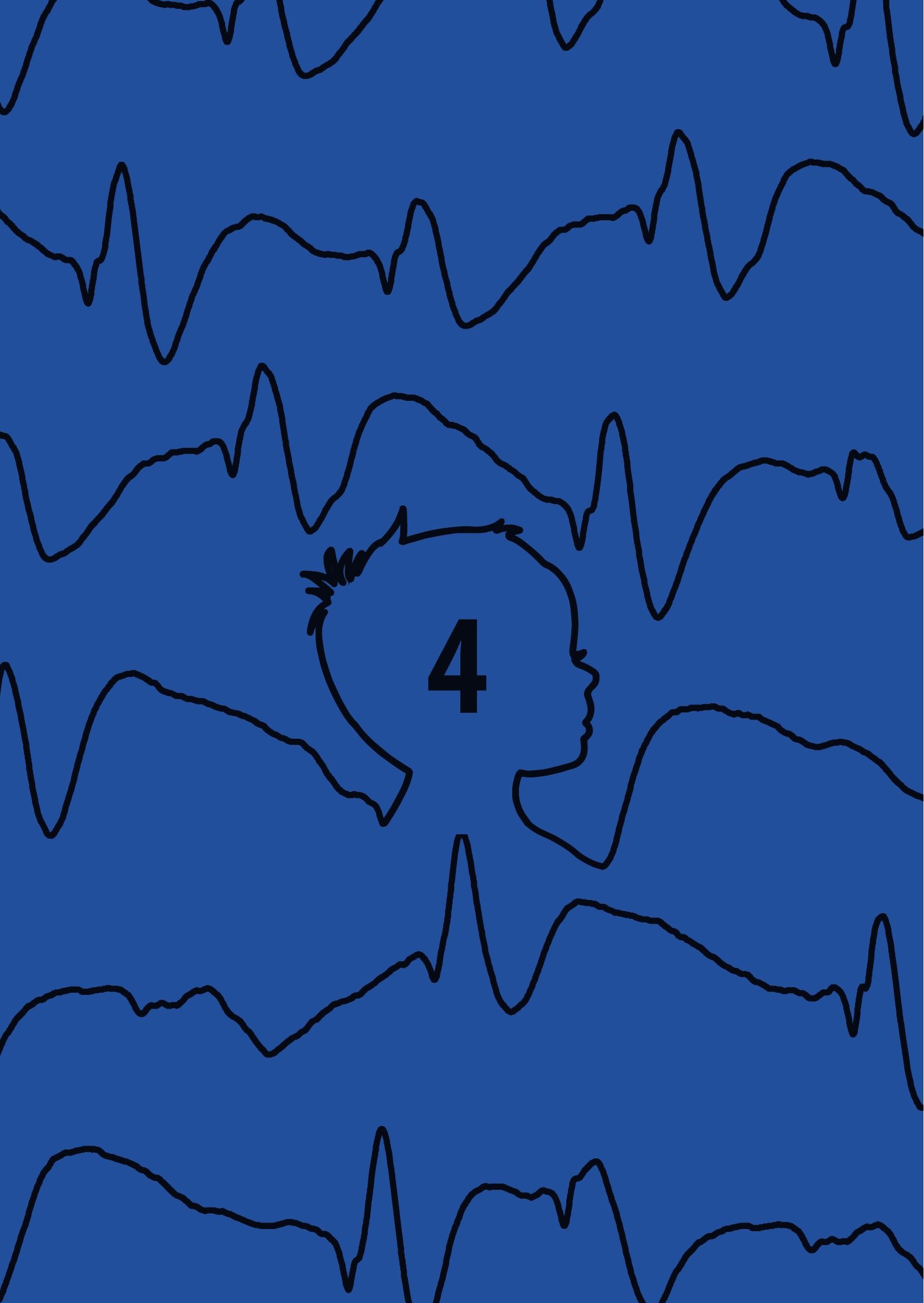


\title{
Chapter 4
}

\section{Serum inflammatory mediators correlate with disease activity in Electrical Status Epilepticus in Sleep (ESES) syndrome}

\author{
Bart van den Munckhof* \\ Evelien E de Vries* \\ Kees PJ Braun \\ $\mathrm{H}$ Myrthe Boss \\ Michèl A Willemsen \\ Annet van Royen-Kerkhof \\ Wilco de Jager** \\ Floor E Jansen** \\ * These first authors contributed equally \\ ** These last authors contributed equally
}

Epilepsia. 2016; 57: e45-50 


\section{Summary}

We aimed to study serum cytokine levels in 11 ESES patients and 20 healthy control children. Patients showed significantly higher levels of IL-1a, IL-6, IL-10, CCL2 and CXCL8/ IL-8 than controls, while MIF and CCL3 were significantly lower. Follow-up analyses in five patients revealed a significant decrease of IL-6 levels after immunomodulating treatment. IL- 6 changes were accompanied by clear improvement of EEG patterns and neuropsychological evaluation. We hypothesize that IL-6 correlates with disease activity and immunomodulating treatment efficacy. 


\section{Introduction}

Encephalopathy with Electrical Status Epilepticus in Sleep (ESES) is a rare epilepsy syndrome that occurs in childhood, and accounts for approximately $0.4-1.3 \%$ of all children with epilepsy. ${ }^{1}$ ESES describes an EEG pattern characterized by sleep induced near-continuous spike-wave discharges. ${ }^{2}$ The electroclinical syndrome is associated with an acquired global cognitive, behavioral or language regression that has its onset between two and twelve years of age. ${ }^{\prime}$ Seizures, occurring in up to $80 \%$ of patients, and EEG epileptiform activity tend to resolve spontaneously during puberty, whereas cognitive impairment often remains despite treatment.

The aim of treatment is to control seizures, abolish the epileptiform EEG activity, but most of all improve cognitive outcome. Conventional and new anti-epileptic drugs may successfully treat seizures, while their effect on cognitive functioning and EEG pattern is often disappointing. ${ }^{2}$ Benzodiazepines are slightly more effective than antiepileptic drugs, especially in restoring physiological electrographic sleep activity. Immunomodulating drugs such as corticosteroids or intravenous immunoglobulins (IVIG) seem to offer better efficacy than conventional anti-epileptic drugs. ${ }^{3-5}$ The mechanism of action of immunomodulating treatments in childhood epilepsy syndromes is still unknown. However, the fact that these drugs are reported efficacious in ESES patients suggests that activation of the immune system plays a role in ESES syndrome, although the exact relationship - cause or consequence - is not known.

The inflammatory process is mediated by soluble inflammatory proteins, including cytokines and chemokines, which are excreted by immune cells and locally inflamed tissue. Hence, measurements of these proteins in peripheral blood might correlate with disease activity and may be used to monitor treatment efficacy. To our knowledge, only a single study measured serum cytokine levels in patients with ESES and demonstrated elevated serum levels of interleukin(IL)-6. ${ }^{6}$ The objective of the present study was to identify markers which correlate with disease activity, and to assess treatment efficacy of immunosuppressive therapy in relation to cytokine levels. To this end we assessed a panel of 16 cytokines in serum of 11 ESES patients and compared these to levels in 20 healthy control children. 


\section{Methods}

\section{Patient selection}

Patients were included at the University Medical Center Utrecht and University Medical Center St. Radboud in Nijmegen. The inclusion criteria for the present study were a diagnosis of ESES between two and twelve years of age, as determined after sleepdeprivation or 24-hour EEG recording in combination with acquired deficits in cognition, language, or behavior (regression or arrest). To determine baseline serum cytokine levels, serum samples were obtained simultaneously with routine laboratory investigations and stored at $-80^{\circ} \mathrm{C}$ until analysis. Patients were treated with oral prednisolone (2 mg/ $\mathrm{kg} /$ day for eight weeks and tapered thereafter), intravenous methylprednisolone (15 $\mathrm{mg} / \mathrm{kg}$ during three days, every four weeks), or intravenous immunoglobulins (IVIG, $2 \mathrm{~g} /$ kg for two days, every four weeks). Healthy children who underwent urological surgery for anatomical deficits served as controls. Blood serum samples in these children were obtained under general anesthesia. This retrospective study was approved by the medical ethics committee of the University Medical Center Utrecht who judged that the "Medical Research Involving Human Subjects Act" did not apply.

\section{Cytokine determination}

Multiplex immunoassay (xMAP; Luminex) was used to measure baseline levels of serum IL$1 a, I L-1 \beta, I L-2, I L-4, I L-5, I L-6, I L-10, I L-12 p 70, I L-13, I L-18$, interferon- $\gamma$ (IFN- $\gamma)$, tumor necrosis factor-a (TNF-a), macrophage inhibitory factor (MIF), and the chemokines CCL2, CCL3 and CXCL8/IL-8, as a brief literature search revealed potential of these markers. In five patients, serum cytokine measurement was repeated approximately six months after inclusion.

\section{Treatment effect}

Treatment effect was assessed for cognition as well as EEG patterns. Improvement of cognition was defined by judgment of the treating physician, and based on history taken from the parents. Improvement of EEG patterns was defined by at least 25\% decrease in spike and wave index (SWI). SWIs were calculated during the first 10 minutes of NREM sleep ( 5 minutes after alpha attenuation) by counting the number of seconds that presented epileptiform activity, divided by 600 , multiplied by 100 to express the amount of epileptiform activity as a percentage.

\section{Statistical analyses}

The Mann-Whitney $U$ test was used to determine a possible difference in cytokine levels between patients and controls, while the Wilcoxon rank sum test was used to evaluate the difference in cytokine levels and SWI before and after immunomodulating treatment. To assess the strength of association between baseline cytokine levels and treatment effect, 
Pearson's correlation coefficient was calculated. All statistical analyses were performed using SPSS Statistics 20 (IBM). P-values below 0.05 were considered statistically significant.

\section{Results}

The study group consisted of 11 ESES patients (age range 2.2-11.8 years, median 5.2 years) and 20 controls (age range 3.7-14.1 years, median 6.8 years). Patient characteristics, both at baseline and follow-up are listed in table 1. Of these patients, four were male, eight had seizures, in three a structural cause of ESES was diagnosed and two had already received methylprednisolone treatment (one and three cycles, respectively) at the time of the first inflammatory protein measurement.

Figure 1A shows the baseline protein levels in patients and controls (values are provided in supplementary table 1). In the total patient group ( $n=11)$, levels of IL-1a, IL-6, IL-10, CCL2 and CXCL8/IL-8 were significantly increased, while MIF and CCL3 levels were significantly decreased compared to controls. Similar results were found in steroid naïve ESES patients ( $n=9$, excluding patients 9 and 10) compared to controls. In addition, the steroid naïve subgroup showed significant differences for IL-5, IL-12p70 and TNF-a.

After Bonferroni correction, IL-10, MIF, CCL2 and CXCL8/IL-8 levels remained significantly different in ESES patients $(n=11)$ compared to controls. IL-10 and CXCL8/IL-8 revealed highest discriminating power, as for both proteins six out of eleven ESES patients fell outside the range of control values. Of note, patient two represented the highest values for IL-1a, IL-6 and TNF-a. This patient had a severe phenotype with severe cognitive impairment, complete dependency in activities of daily living and autism spectrum disorder, and had tonic-clonic seizures as well as atypical absence seizures. However, since other patients had similar clinical characteristics we feel that phenotype alone did not explain the high protein levels. In summary, in serum of ESES patients, levels of IL-1a, IL-6, IL-10, CCL2 and CXCL8/IL-8 were increased, with IL-10 and CXCL8/IL-8 showing highest discriminating power, while levels of MIF and CCL3 were decreased.

To determine the value of inflammatory mediators in relation to treatment efficacy, the cytokine levels offive patients that were also measured after start of the immunomodulating treatment (figure 1B) were compared to baseline levels. Three out of five patients had been treated with oral prednisone (range: five to nine weeks), while two patients were administered IVIG (both six cycles). IL-6 levels decreased by at least seven-fold in all five patients after immunomodulating treatment $(P=0.04)$, while a decrease in TNF- $a$ and CCL2 levels after treatment was just not significant. Interestingly, the decrease in cytokine 
Chapter 4. Inflammatory mediators in ESES syndrome

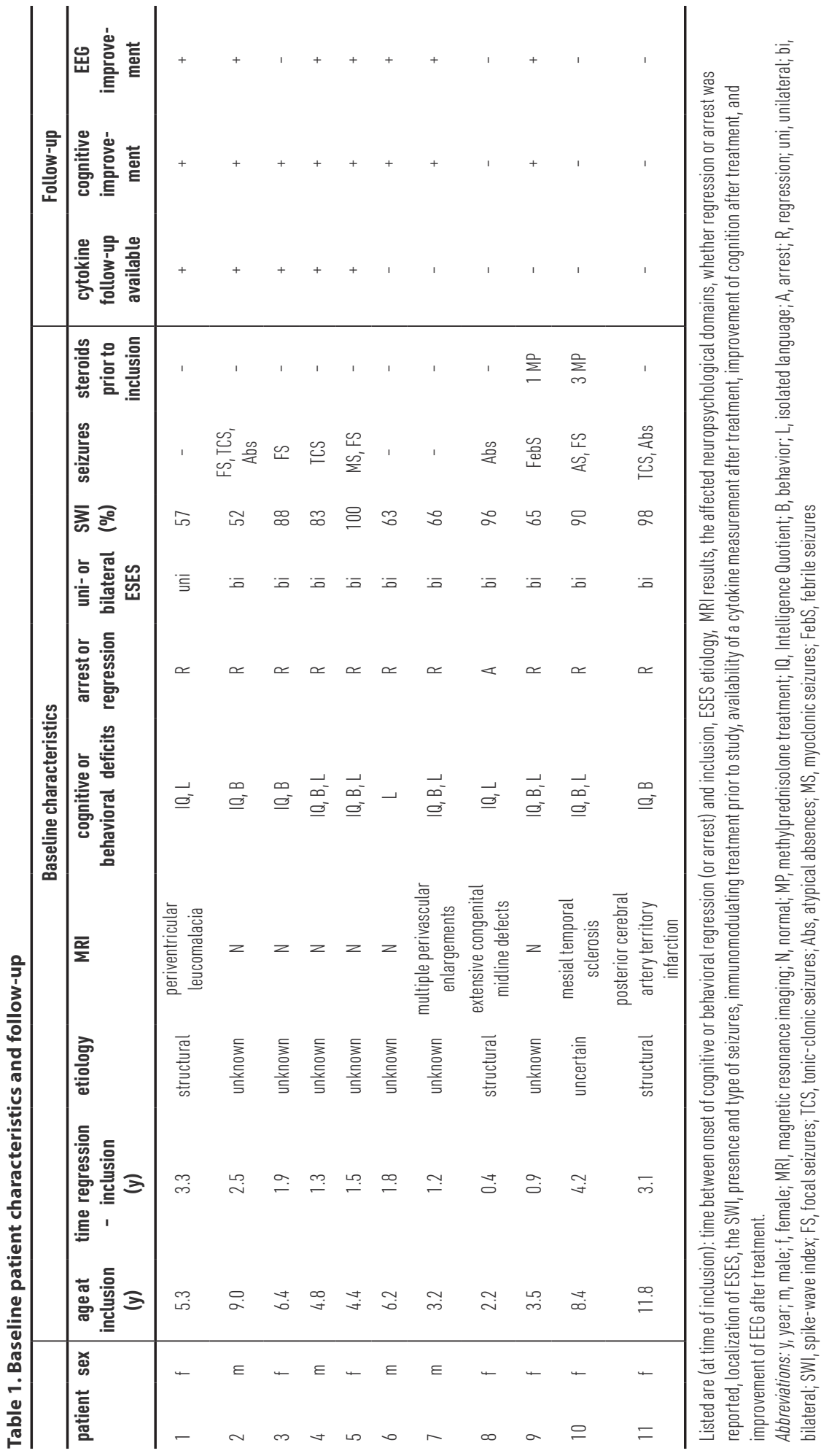



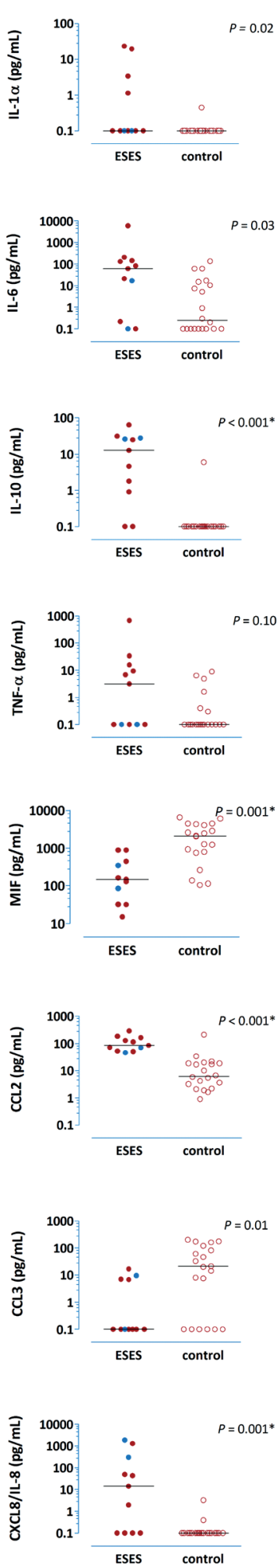
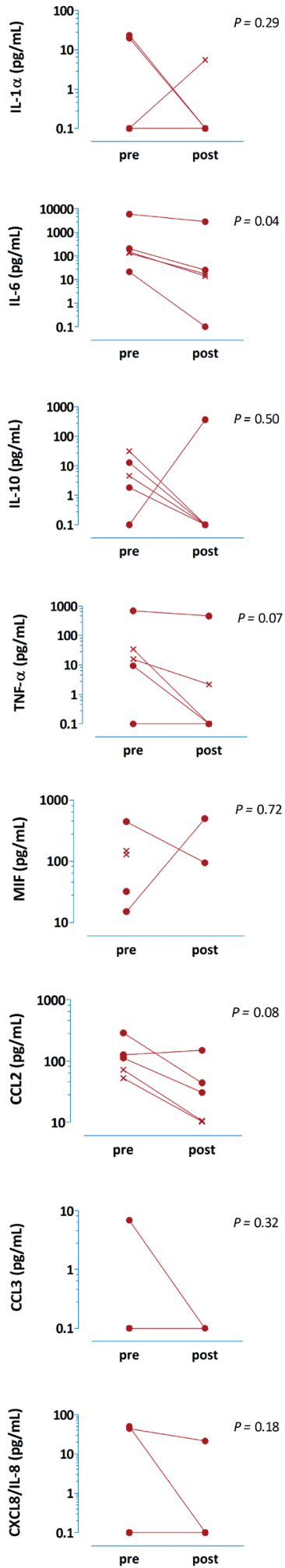

Figure 1. (A) Scatter plots of baseline cytokine levels of ESES patients and controls.

Patients showed significantly higher levels of IL-1a, IL-6, IL-10, CCL2 and CXCL8/IL-8, and significantly lower levels of MIF and CCL3. Circles represent individual subjects, horizontal lines the median. Blue dots represent two patients who had already received methylprednisolone treatment at inclusion. *: remained significant after Bonferroni correction. (B) Cytokine levels over time in five patients before (pre) and after (post) immunomodulating treatment. Each line represents one patient. Circles represent three patients who had received oral prednisone treatment for eight weeks, crosses represent two patients who had received six cycles of IVIG. For patients receiving IVIG, no follow-up of MIF and CCL3 was available after six cycles. Levels of IL-6 decreased significantly over time, whereas levels of TNF- $a$ and CCL2 tended to decrease. 
Chapter 4. Inflammatory mediators in ESES syndrome

levels was accompanied by a clear improvement of EEG patterns, with a significant overall drop in SWI of $77 \%$ ( $P=0.04$, supplementary table 2$)$. Furthermore, after six months of immunomodulating treatment, all five patients showed distinct improvement of language and behavior as assessed with neuropsychological evaluation. These findings suggest that serum levels of IL-6 correlate with immunomodulating treatment efficacy. Because all patients in our study showed improvement in cognition or EEG, it remains uncertain whether there is a true correlation between a decrease of cytokine levels and clinical or EEG improvement.

Calculation of correlation between baseline cytokine levels in 11 patients and immunomodulating treatment efficacy, as measured by cognitive or EEG improvement, revealed no significance (supplementary table 3).

\section{Discussion}

This study demonstrates that in serum of ESES patients, levels of IL-1a, IL-6, IL-10, CCL2 and CXCL8/IL-8 were increased, while levels of MIF and CCL3 were decreased compared to controls. Because cytokine levels can be influenced by corticosteroid treatment, ${ }^{7}$ we also investigated a subgroup of steroid-naïve patients and compared their cytokine levels to control levels. In this subgroup we found the same cytokines to be significantly different and in addition levels of IL-5, IL-12p70 and TNF-a were found to be significantly different from control levels. Furthermore, IL-6 levels were significantly decreased, a sevenfold reduction, six months after start of immunomodulating treatment, while TNF-a and CCL2 levels tended to diminish. Importantly, IL-6 levels correlated with positive treatment response, assessed by decrease in SWI and ameliorating outcomes of neuropsychological evaluation.

Interleukin-6 is a general marker of inflammation and is secreted by monocytes and macrophages to stimulate the immune response. Persistent dysregulated expression of IL-6 has been implicated in the pathophysiology of several autoimmune diseases, such as systemic juvenile idiopathic arthritis and large-vessel vasculitis, and blockade therapy of IL-6 has been proven effective in these disorders. ${ }^{8} \mathrm{IL}-6$ is hypothesized to be involved in seizure mechanisms as well, since administration of IL-6 in rats worsened seizure activity and lowered seizure threshold ${ }^{9}$ and multiple studies revealed elevated IL-6 levels in people with various epilepsy related etiologies..$^{10-12}$ The observed increase in IL-6 levels in serum of ESES patients in the present case series is consistent with the previous study. ${ }^{6}$ We found a subsequent decrease after the immunomodulating treatment. Therefore, we hypothesize that IL-6 is involved the pathophysiology of ESES syndrome and might prove 
valuable when considering targeted treatment. Although seizures are often infrequent or of minimal burden in ESES patients, it would be valuable to evaluate a possible relation between seizure frequency and IL-6 levels in future studies.

Furthermore, we demonstrated increased levels of CCL2 in ESES patients, which tended to decrease after start of immunomodulating treatment (the latter was also found for TNF-a). We hypothesize that the differences between pre- and post-treatment TNF- $a$ and CCL2 values did not reach significance due to the limited number of ESES patients, and suggest future studies to further explore the correlation of TNF-a and CCL2 with ESES disease activity.

It is unclear whether increased cytokine levels precede ESES onset, which may suggest a causative role in the onset of the disorder, or whether they are an epiphenomenon, and the decrease after immunomodulating treatment is merely a parallel to clinical improvement. Studies investigating other epilepsy disorders demonstrated that administration of several pro-inflammatory cytokines to rodents worsened seizure activity and lowered seizure threshold. ${ }^{9,13}$ Investigating the causative role of inflammation in the pathophysiology of ESES syndrome is hampered by the absence of an accurate animal model, the rarity and high heterogeneity of this disorder. This study is the first to assess a larger panel of cytokines in ESES patients.

This study compares ESES patients to healthy controls of comparable age. A limitation of the study is the small sample size, which made it difficult to account for bias. Also the follow-up was incomplete due to lack of necessity of second routine laboratory investigations in six of eleven patients. Furthermore, if a lumbar puncture is considered clinically indicated, we would recommend future studies to measure inflammatory mediators in cerebrospinal fluid (or brain tissue of surgically treated patients) as well, since that reduces the risk of measuring peripheral inflammatory processes.

In summary, we found that multiple cytokine levels were increased or decreased in ESES patients, which suggests that the immune system is actively involved in the pathophysiology of ESES syndrome. Furthermore, IL-6 decreased significantly after immunomodulating therapy, while TNF-a and CCL2 showed a trend towards significant change. These findings could indicate a role for targeted anti-cytokine therapy with the ultimate goal to improve cognitive outcome in patients with ESES syndrome.

\section{Acknowledgements}

This work was supported by grants from the Dutch Epilepsy Fund and the Wilhelmina Children's Hospital Research Fund. 


\section{Disclosure of Conflicts of Interest}

Dr. van den Munckhof, Prof. Dr. Braun, and Dr. Jansen report grants from the Dutch Epilepsy Fund and Wilhelmina Children's Hospital Research Fund during the conduct of the study. The funding sources had no involvement in this study. The remaining authors have no conflicts of interest.

We confirm that we have read the Journal's position on issues involved in ethical publication and affirm that this report is consistent with those guidelines. 


\section{References}

1. Sánchez Fernández I, Loddenkemper T, Peters, J, et al. Electrical status epilepticus in sleep: Clinical presentation and pathophysiology. Pediatr Neurol 2012;47:390-410.

2. Nickels K, Wirrell E. Electrical Status Epilepticus in Sleep. Semin. Pediatr Neurol 2008;15:50-60.

3. Buzatu M, Bulteau C, Altuzarra C, et al. Corticosteroids as treatment of epileptic syndromes with continuous spike-waves during slow-wave sleep. Epilepsia 2009;50: 68-72.

4. Kramer U, Sagi L, Goldberg-Stern H, et al. Clinical spectrum and medical treatment of children with electrical status epilepticus in sleep (ESES). Epilepsia 2009;50:1517-1524.

5. Van den Munckhof B, van Dee V, Sagi L, et al. Treatment of Electrical Status Epilepticus in Sleep (ESES): a pooled analysis of 575 cases. Epilepsia (Epub 2015 Sep 4).

6. Lehtimaki KA, Liimatainen S, Peltola J, et al. The serum level of interleukin- 6 in patients with intellectual disability and refractory epilepsy. Epilepsy Res 2011;95:184-187.

7. Rhen T, Cidlowski JA. Antiinflammatory Action of Glucocorticoids -New Mechanisms for Old Drugs. N Engl J Med 2005;353:1711-1723.

8. Kang S, Tanaka T, Kishimoto T. Therapeutic uses of anti-interleukin-6 receptor antibody. Int Immunol 2014;27:21-29.

9. Kalueff A, Lehtimaki, KA, Ylinen A, et al. Intranasal administration of human IL-6 increases the severity of chemically induced seizures in rats. Neurosci Lett 2004;365:106-10.

10. Liimatainen S, Fallah M, Kharazmi E, et al. Interleukin-6 levels are increased in temporal lobe epilepsy but not in extra-temporal lobe epilepsy. J Neurol 2009;256:796-802.

11. Choi J, Min HJ, Shin JS. Increased levels of HMGB1 and pro-inflammatory cytokines in children with febrile seizures. J Neuroinflammation 2011;8:135.

12. Shu HF, Zhang CQ, Yin Q, et al. Expression of the interleukin 6 system in cortical lesions from patients with tuberous sclerosis complex and focal cortical dysplasia type Ilb. J Neuropathol Exp Neurol 2010;69:838-849.

13. Vezzani A, Moneta D, Conti M, et al. Powerful anticonvulsant action of IL-1 receptor antagonist on intracerebral injection and astrocytic overexpression in mice. Proc Nat Acad Sci USA 2000;97:115349. 


\section{Supporting information}

Supplementary table 1. Baseline cytokine levels

\begin{tabular}{|c|c|c|c|c|c|}
\hline \multirow[b]{2}{*}{ analyte } & \multicolumn{3}{|c|}{ analyte concentration (pg/mL) } & \multicolumn{2}{|c|}{ P-value } \\
\hline & ESES $(n=11)$ & $\begin{array}{c}\text { ESES } \\
\text { (steroid } \\
\text { naïve, } n=9 \text { ) }\end{array}$ & $\begin{array}{l}\text { control } \\
(n=20)\end{array}$ & ESES vs control & $\begin{array}{c}\text { ESES (steroid naïve) } \\
\text { vs control }\end{array}$ \\
\hline IL-1a & $4.3 \pm 8.6$ & $5.3 \pm 9.3$ & $0.0 \pm 0.1$ & 0.02 & 0.007 \\
\hline IL-1 $1 \beta$ & $1.1 \pm 3.7$ & $1.4 \pm 4.1$ & $1.2 \pm 2.7$ & 0.23 & 0.37 \\
\hline IL-2 & $0.9 \pm 3.0$ & $1.1 \pm 3.4$ & $0.1 \pm 0.3$ & 0.52 & 0.67 \\
\hline IL-4 & $0.0 \pm 0.0$ & $0.0 \pm 0.0$ & $0.0 \pm 0.0$ & 1.00 & 1.00 \\
\hline IL-5 & $1.0 \pm 2.3$ & $1.2 \pm 2.5$ & $0.0 \pm 0.0$ & 0.05 & 0.03 \\
\hline IL-6 & $599.4 \pm 1768.2$ & $730 \pm 1949.8$ & $15.8 \pm 34.0$ & 0.03 & 0.01 \\
\hline IL-10 & $17.6 \pm 19.7$ & $15.5 \pm 21.4$ & $0.3 \pm 1.3$ & $<0.001$ & $<0.001$ \\
\hline IL-12p70 & $17.7 \pm 28.8$ & $21.7 \pm 30.7$ & $7.5 \pm 10.9$ & 0.13 & 0.03 \\
\hline IL-13 & $5.2 \pm 11.6$ & $6.3 \pm 12.7$ & $1.9 \pm 7.4$ & 0.17 & 0.08 \\
\hline IL-18 & $112.3 \pm 58.3$ & $113.2 \pm 64.6$ & $132.9 \pm 128.3$ & 0.80 & 0.81 \\
\hline IFN-ү & $49.6 \pm 149.6$ & $60.6 \pm 165.0$ & $104.1 \pm 247.5$ & 0.57 & 0.83 \\
\hline TNF-a & $68.6 \pm 204.9$ & $83.8 \pm 225.9$ & $1.1 \pm 2.5$ & 0.10 & 0.03 \\
\hline MIF & $285.7 \pm 320.7$ & $301.5 \pm 350.4$ & $2363.1 \pm 1987.2$ & 0.001 & 0.002 \\
\hline CCL2 & $113.5 \pm 74.2$ & $125.9 \pm 76.8$ & $20.1 \pm 44.9$ & $<0.001$ & $<0.001$ \\
\hline CCL3 & $3.7 \pm 5.7$ & $3.4 \pm 5.9$ & $56.1 \pm 69.9$ & 0.01 & 0.01 \\
\hline CXCL8/IL-8 & $325.3 \pm 639.0$ & $157.0 \pm 429.9$ & $0.2 \pm 0.7$ & 0.001 & 0.005 \\
\hline
\end{tabular}

Baseline cytokine levels in all ESES patients, ESES patients that had not received corticosteroids before their baseline sample and controls (values in mean \pm SD). P-values in bold show significant differences between groups.

\section{Supplementary table 2. Difference in SWI}

\begin{tabular}{|c|c|c|c|c|c|}
\hline patient & $\begin{array}{c}\text { SWI before treatment } \\
(\%)\end{array}$ & $\begin{array}{c}\text { SWI after treatment } \\
(\%)\end{array}$ & $\Delta \mathrm{SWI}(\%)$ & $P$-value & treatment \\
\hline 1 & 57 & 8 & -86 & & prednisone 5 weeks \\
\hline 2 & 52 & 3 & -94 & & prednisone 6 weeks \\
\hline 3 & 88 & 73 & -17 & & IVIG 3 cycles \\
\hline 4 & 83 & 6 & -93 & & $\begin{array}{c}\text { IVIG } 6 \text { cycles + prednisone } 8 \\
\text { weeks }\end{array}$ \\
\hline \multirow[t]{2}{*}{5} & 100 & 7 & -93 & & prednisone 9 weeks \\
\hline & $76 \pm 21$ & $19 \pm 30$ & $-77 \pm 33$ & 0.04 & \\
\hline
\end{tabular}

The difference in SWI ( $\triangle$ SWI) before and after immunomodulating treatment (bottom values in mean \pm SD). The SWI of patient 4 did not respond to IVIG alone, therefore this patient received 8 weeks of oral prednisone subsequent to IVIG.

Abbreviations: SWI, spike-wave index; IVIG, intravenous immunoglobulins. 
Supplementary table 3. Correlation baseline cytokine levels and treatment efficacy

\begin{tabular}{|c|c|c|}
\hline \multirow[b]{2}{*}{ analyte } & \multicolumn{2}{|c|}{ Pearson's correlation coefficient } \\
\hline & $\begin{array}{l}\text { improvement cognition } \\
(\mathrm{n}=11)\end{array}$ & $\begin{array}{l}\text { improvement EEG } \\
(n=11)\end{array}$ \\
\hline IL-1a & 0.54 & 0.38 \\
\hline IL-1B & 0.59 & 0.49 \\
\hline IL-2 & 0.57 & 0.48 \\
\hline IL-4 & 0.10 & 0.20 \\
\hline IL-5 & 0.24 & 0.41 \\
\hline IL-6 & 0.52 & 0.45 \\
\hline IL-10 & 0.40 & 0.72 \\
\hline IL-12p70 & 0.30 & 0.26 \\
\hline IL-13 & 0.99 & 0.79 \\
\hline IL-18 & 0.82 & 0.76 \\
\hline IFN- $\gamma$ & 0.53 & 0.51 \\
\hline TNF-a & 0.53 & 0.49 \\
\hline MIF & 0.60 & 0.84 \\
\hline CCL2 & 0.16 & 0.09 \\
\hline CCL3 & 0.66 & 0.43 \\
\hline CXCL8/LL-8 & 0.52 & 0.37 \\
\hline
\end{tabular}

Pearson's correlation coefficient ( $P$-value) for relation between baseline cytokine levels and immunomodulating treatment efficacy (assessed for both cognition as well as EEG patterns). 

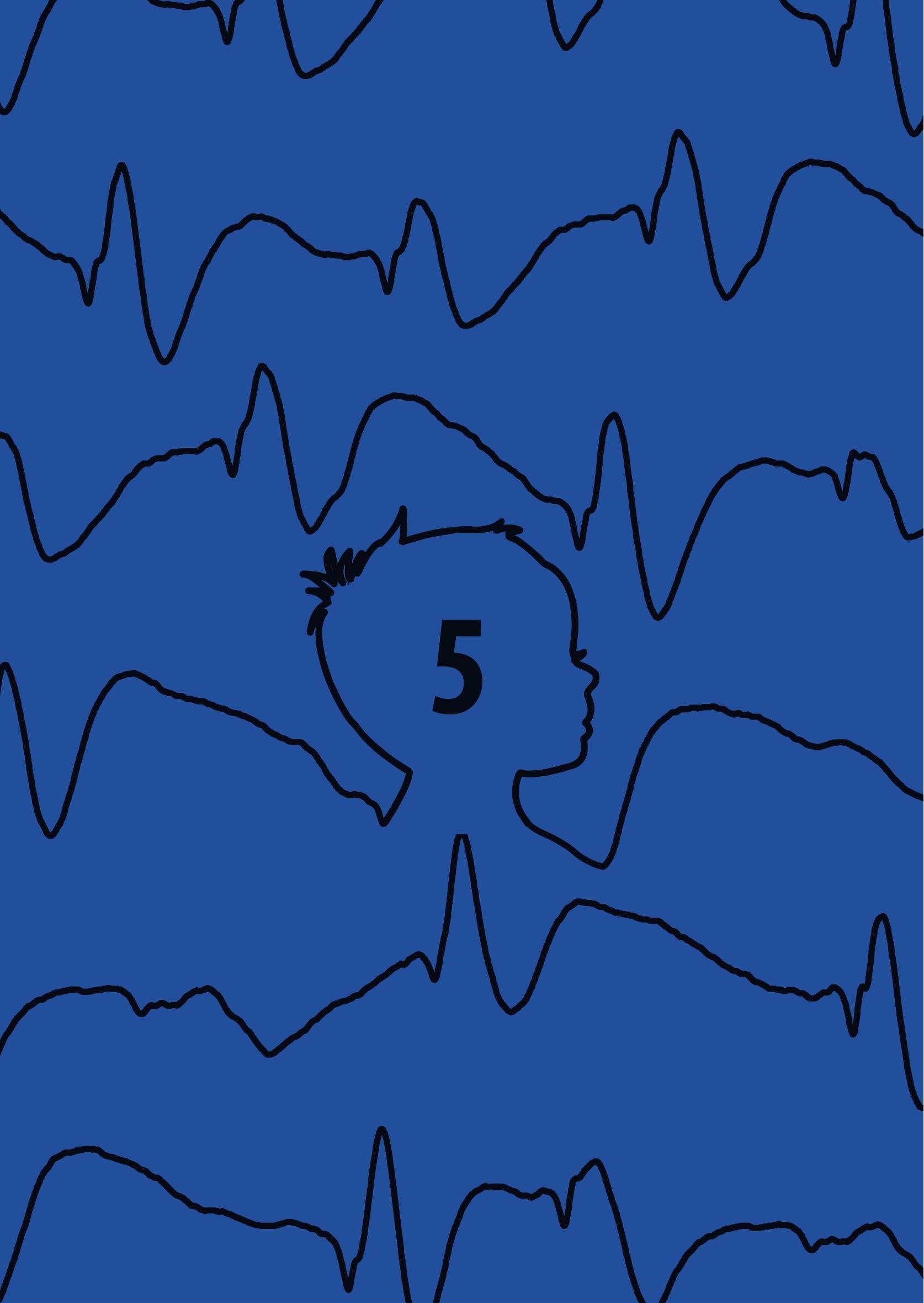
Chapter 5

\section{Sleep slow wave homeostasis and cognitive functioning in children with electrical status epilepticus in sleep}

Bart van den Munckhof

Silvano R. Gefferie

Suus A.M. van Noort

Heleen C. van Teeseling

Mischa P. Schijvens

William Smit

Nico W. Teunissen

* These last authors contributed equally.

\author{
Joost D.J. Plate \\ Geert Jan M. Huiskamp \\ Frans S.S. Leijten \\ Kees P.J. Braun \\ Floor E. Jansen* \\ Bigna K. Bölsterli*
}




\section{Abstract}

Study Objectives: Encephalopathy with electrical status epilepticus in sleep (ESES) is characterised by non-rapid eye movement (non-REM)-sleep-induced epileptiform activity and acquired cognitive deficits. The synaptic homeostasis hypothesis describes the process of daytime synaptic potentiation balanced by synaptic downscaling in non-REM-sleep and is considered crucial to retain an efficient cortical network. We aimed to study the overnight decline of slow waves, an indirect marker of synaptic downscaling, in patients with ESES and explore whether altered downscaling relates to neurodevelopmental and behavioural problems.

Methods: Retrospective study of patients with ESES with at least one whole-night electroencephalogram (EEG) and neuropsychological assessment (NPA) within 4 months. Slow waves in the first and last hour of non-REM-sleep were analysed. Differences in slow wave slope (SWS) and overnight slope course between the epileptic focus and non-focus electrodes and relations to neurodevelopment and behaviour were analysed.

Results: 29 patients with 44 EEG NPA combinations were included. Mean SWS decreased from 357 to $327 \mu \mathrm{V} / \mathrm{s}(-8 \%, \mathrm{p}<0.001)$ across the night and the overnight decrease was less pronounced in epileptic focus than in non-focus electrodes (-5.6\% vs. $-8.7 \%, p=0.003)$. We found no relation between SWS and neurodevelopmental test results in cross-sectional and longitudinal analyses. Patients with behavioural problems showed less SWS decline than patients without and the difference was most striking in the epileptic focus $(-0.9 \%$ vs. $-8.8 \%, p=0.006$ ).

Conclusion: Slow wave homeostasis - a marker of synaptic homeostasis - is disturbed by epileptiform activity in ESES. Behavioural problems, but not neurodevelopmental test results, were related to severity of this disturbance.

\section{Statement of significance}

The hallmark of encephalopathy with ESES is near-continuous epileptiform activity in slow wave sleep, accompanied by neuropsychological disturbances. Slow wave sleep is of known importance for cognitive functioning. The synaptic homeostasis hypothesis proposes a framework for the role of slow wave sleep in cognitive functioning. With this study, we analysed whether slow waves are related to neurodevelopmental and behavioural functioning in children with ESES. We found that an impaired overnight decline in slow wave slope is related to behavioural problems. Disrupted slow wave homeostasis might link epileptiform activity in non-REM sleep to neuropsychological 
problems. Unravelling the mechanisms behind neuropsychological deficits, may lead to a better understanding of this epileptic encephalopathy and how disturbances of sleep physiology impact cognition.

\section{Introduction}

Epileptic encephalopathy with electrical status epilepticus in sleep (ESES) is a rare childhood epilepsy syndrome characterised by near-continuous epileptiform activity during non-REM-sleep associated with neuropsychological deficits. Patients usually present with a global cognitive, language or behavioural regression between 2 and 12 years of age. A majority of patients has (occasional) seizures, often with onset before the occurrence of developmental deficits 1-3. ESES can be diagnosed in the context of structural (especially when involving the thalamus or perisylvian region) or genetic abnormalities (e.g. a GRIN2A mutation), but in about one third of cases the aetiology is unknown 4-8. Seizures generally respond well to anti-epileptic drug treatment, while the treatment of cognitive symptoms is challenging. That ESES leads to cognitive decline is accepted, but how is incompletely understood. The spike-wave index (SWI), reflecting the proportion of non-REM-sleep affected by epileptiform discharges, is often used as an EEG marker of ESES severity 9, with EEG abnormalities in typical ESES often defined as bilateral and with an SWI above 85\%, while atypical cases with an SWI of 50-85\% are also considered part of the spectrum. The SWI is also used to evaluate of treatment efficacy. However, previous studies have shown that it does not always correlate to the severity of neurodevelopmental deficits and that in a substantial part of the patients there is a discrepancy between SWI and cognitive response to treatment 10-13.

The crucial role of sleep - in particular slow wave sleep - for cognitive functioning is well known and illustrated by the synaptic homeostasis hypothesis 14. This hypothesis states that during wakefulness, through active thinking and exposure to novel experiences and sensory stimuli, people learn. On a cellular level, this is reflected by synaptic potentiation: an increase in number and strength of connections between neurons. During sleep, the brain is functionally detached from external stimuli and a recuperative process named synaptic downscaling is suggested to take place. The (partially selective) reduction in the number and strength of synaptic connections during sleep is essential because it restores net synaptic strength to an efficient baseline level. The overnight reduction in synaptic connections thus provides space and saves energy for another increase in synaptic connections during the following day 15-17. The process of synaptic homeostasis is, based on both animal and human studies, thought to be reflected, and possibly induced, by slow wave activity (SWA) 17-19. Slow waves are delta (0.5 - 4 Hertz) waves seen in the 
Chapter 5. Sleep slow waves and cognition in children with ESES

electroencephalogram (EEG) during non-REM sleep, caused at the cellular level by nearsynchronous oscillations of large groups of neurons 20,21. The slow wave amplitude and slope are closely related to the firing synchronicity or efficacy of neuronal interaction and thus provide markers of synaptic strength 18,19,22,23. In healthy people, SWA decreases with sleep; while slow waves in non-REM sleep have a relatively high amplitude and slope at sleep onset, they are much lower towards the end of the night 19.

The hypothesis that slow waves are related to cognitive functioning has been explored by several studies. In healthy subjects, performance of a specific motor learning task just before sleep was associated with local increases in slow wave activity in the region used in this task in the following period of sleep and a subsequent improvement in task performance after sleep ${ }^{24}$. In addition, boosting slow waves by auditory closed-loop stimulation, enhanced performance on an overnight memory task ${ }^{25}$. In a study in patients with epilepsy, seizures and interictal spikes increased slow wave activity in the epileptic focus and reduced the homeostatic overnight slow wave decline as well as visual learning abilities during wakefulness ${ }^{26}$.

Several studies have suggested that the near-continuous epileptiform activity during non-REM sleep in patients with ESES interferes with learning and memory consolidation by disturbing physiological slow wave activity ${ }^{27-29}$. Evidence for impaired slow wave homeostasis was found in a study comparing the overnight course of slow wave characteristics between 9 ESES patients and 9 healthy controls. While the controls showed a significant reduction in slow wave amplitude and slope across the night, this was not seen in the ESES patients ${ }^{30}$. In a subsequent study, the slow wave slope change across the night was found to be correlated to the SWI ${ }^{31}$. Furthermore, the slow wave slope at the end of the night was steeper in the electrodes at the epileptic focus as compared to the "non-focal" electrodes. After remission of ESES, the slow wave homeostasis normalized and in an exploratory analysis, the cognitive sequelae seemed larger in children with the most impaired slow wave homeostasis during their active ESES phase ${ }^{32}$.

To our knowledge, the possible relation between slow wave parameters and neuropsychological functioning has not been assessed in the population of ESES patients.

With the current study we explored: 1. whether there is a difference in overnight slow wave course (slow wave homeostasis) between the electrodes in the epileptic focus and the non-focus electrodes. Based on previous studies 31,32, we hypothesized that the slow wave slope decline in the epileptic focus would be less marked or absent as compared to the non-focus electrodes; 2 . the relation between slow wave homeostasis and neurodevelopment and behaviour across patients with ESES. We hypothesised that 
patients with a (more pronounced) decline of slow wave slope across the night have relatively preserved neuropsychological functioning and normal behaviour as compared to those with severely impaired slow wave homeostasis; and 3. the relation between a change in the slow wave homeostasis and a change in neuropsychological functioning in an exploratory longitudinal comparison within ESES patients. We expected that an improvement in slow wave decline across the night over time would be associated with an improvement in cognitive functioning and behaviour.

\section{Methods}

\section{Patient selection}

We retrospectively included all patients with at least one whole night sleep EEG and a neuropsychological assessment (NPA) performed between January 2009 and July 2017 at the University Medical Center Utrecht, if they fulfilled the following criteria:

- at least one whole night sleep EEG showing an ESES pattern, defined as a spike wave index in sleep of at least $50 \%$ and at least $25 \%$ aggravation in comparison to wakefulness

- acquired cognitive or behavioural deficits; either new deficits or alteration of the expected developmental trajectories.

- at least one EEG / NPA combination fulfilling the selection criteria below

Combinations of EEG and NPA were included if:

- interval between EEG and NPA $<4$ months

- age below 12 years at the time of these investigations

- no more than one (clinical or electrophysiological) seizure during the whole night registration

The study was approved by the medical ethics committee who judged that the Dutch Medical Research Involving Human Subjects Act did not apply.

\section{Patient characteristics}

Baseline patient characteristics (gender; age at ESES diagnosis; aetiology, categorized as structural, genetic / syndromal, post-infectious or unknown; MRI findings; seizure frequency and development before ESES diagnosis) were collected. For continuous variables that follow a normal distribution, means and standard deviations are reported, whereas for not normally distributed variables median and range are reported. 


\section{Neuropsychological findings}

For each neuropsychological assessment the type of test and test results were collected. A type of test was chosen and adapted to the age and abilities of the child. Depending on the tests performed, we extracted total intelligence quotient (TIQ), developmental quotient (DQ), verbal IQ (VIQ), performance IQ (PIQ), processing speed, Beery test of visuomotor integration (VMI), digit span (memory) and Peabody Picture vocabulary test (PPVT) from the reports and test forms. For patients with severe cognitive impairment, in whom full intelligence testing was not possible, mental age and developmental quotient were calculated. Development before ESES onset and behavioural problems (e.g. mood regulation problems, anxiety) were categorized as absent, mild, moderate or severe in concordance with criteria used by Massa et al ${ }^{33}$. Patients with a pre-existing developmental delay could only be included if they had a clear regression or arrest of development on top of this. This deviation from expected developmental trajectories was preferably shown by a repeat NPA (if they already had an NPA before) or otherwise based on parental history as taken by the paediatric neurologist and neuropsychologist. Behaviour was assessed by the neuropsychologist using a structured parental interview, often supplemented by a behavioural questionnaire (the Child Behaviour Checklist, $\mathrm{CBCL}$ ). Behavioural abnormalities were first categorised as absent, mild, moderate or severe in concordance with criteria used by Massa et al. 2001. Considering the relatively small number of measurements, we subsequently categorised this as normal / abnormal (mild, moderate or severe behavioural abnormalities were thus all recoded as abnormal). This allowed statistically comparing slow wave slope course between two groups of reasonable size.

\section{EEG acquisition and pre-processing}

Scalp EEGs with 21 electrodes according to the international 10-20 / 10-10 system and electrooculograms were recorded. In most cases chin electromyography was also available and in some cases measurements of respiratory effort. For each included EEG a spike wave index (SWI) was calculated in an epoch of 10 minutes (600 s), starting 5 minutes after alpha attenuation or, in absence of alpha rhythm, after sleep had commenced based on video and EEG features suggestive of sleep onset. The number of seconds containing epileptiform discharges at any of the electrodes was divided by the total number of seconds in the epoch (600) and multiplied by 100 to reflect the SWI as a percentage. In addition, the background pattern, presence or absence of physiological sleep phenomena and localisation (focus) of epileptiform abnormalities were assessed. EEG epochs were scored as wake, non-REM, REM or movement / artefacts according to the American Academy of Sleep Medicine (AASM) Manual for the Scoring of Sleep and Associated Events, version 2.3 (2016), if possible. If this was not possible because of a lack of recognisable sleep architecture, the modified criteria used in previous studies with ESES patients were 
used 30-32. EEG channels for which the signal consisted of mainly artefacts (e.g. channels that were lost during the night), were removed from the analysis.

\section{Calculation of slow wave characteristics}

We used data processing algorithms for the detection of sleep slow waves $(0.5-2 \mathrm{~Hz})$, spikes and artefacts, based on previously published methods 19,30. First, sleep slow waves of the first hour (FH) and last hour ( $\mathrm{LH}$ ) of artefact-free non-REM sleep were automatically detected in each electrode. Subsequently, spikes were detected. Then, slow waves that occurred within a time window of 0.5 seconds after a spike were considered part of a spike-wave complex and thus excluded from the analysis. The amplitudes of the included slow waves were assessed. The absolute slopes of the included slow waves were calculated as the amplitude of the negative peak divided by the time interval between the negative peak and the next zero-crossing, i.e. the ascending slope. To account for variability in amplitude of individual slow waves (also influenced by age and technical aspects) which inherently would influence the calculated slope - the slope was also calculated at a set amplitude of $75 \mu \mathrm{V}$, as in previous studies 30-32. The amplitudes and absolute slope characteristics are described only, while the normalized slope is used as the primary slow wave characteristic in the analyses and further referred to as slope. It was calculated for each electrode for the first and last hour of non-REM sleep (FH slope and LH slope). Relative slope change (FH slope subtracted from LH slope, divided by the FH slope) was calculated for each EEG electrode to reflect the overnight course of slope.

We predefined regions of interest for the analysis of slow wave characteristics: analyses were performed for the mean slow wave characteristics of all electrodes, for the focus electrodes, non-focus electrodes and for default-mode network (DMN) electrodes. The focus was defined as up to four electrodes with epileptiform activity and if more than four electrodes showed epileptiform activity, the four with the highest amplitude spikes were selected. The remaining electrodes were designated as non-focus electrodes. Based on previous studies, the default-mode network was chosen as a region of interest for the relation of slow wave characteristics with neuropsychological functioning 34,35. In accordance with a recent study, the default-mode network included the electrodes recording over the prefrontal cortex (Fp1 and Fp2 electrodes), precuneus (Pz electrode) and bilateral parietal areas (P7 / P8 electrodes).36

\section{Outcome definition}

In the first analysis, comparing the slow wave slope characteristics of the focus versus non-focus electrodes, the outcome was defined as the relative slow wave slope change across the night (normalised at $75 \mu \mathrm{V}$ ). For the cross-sectional and longitudinal analyses of possible relations between slow wave slope characteristics and neuropsychological 
Chapter 5. Sleep slow waves and cognition in children with ESES

functioning, we used the test results of the neuropsychological assessment $(T I Q, V I Q, P I Q$, PS, VMI and digit span) as quantitative outcome measures. Behaviour was included as a qualitative outcome measure (normal / abnormal) at the time of the EEG recording / neuropsychological assessment. We also analysed whether treatment with conventional anti-epileptic drugs (AED), benzodiazepines or steroids at the time of the EEG / NPA was related to behaviour, as this might be a confounding factor in assessing the relation between overnight slow wave slope course and behaviour. Lastly, for the longitudinal analysis, we also used SWI improvement (at least 25\% decrease in SWI) and subjective improvement (improved / not improved) in daily functioning as reported by the parents and the treating physician, as qualitative outcomes.

\section{Statistical analysis}

For all patients, we described slow wave slope course in the predefined regions and analysed possible correlation of the relative slow wave slope difference in these regions with neuropsychological outcome measures in their first EEG NPA combination. Thus, for patients with multiple EEG NPA combinations, only the first combination was used for this analysis. To assess differences in $\mathrm{FH}$ and $\mathrm{LH}$ slow wave slope and to compare overnight slope course between focus and non-focus electrodes, paired samples t-tests were reported. For continuous outcomes, linear regression analysis was performed; for correlation of slow wave characteristics and SWI (not normally distributed), Spearman's Rho was assessed and for dichotomous outcome (behavioural abnormalities), an independent samples t-test or Mann-Withney U-test was used (depending on the distribution of the data). Fisher's exact test was used to assess whether there was an association between the presence or absence of treatment with AED, benzodiazepines and steroids, and behavioural abnormalities.

For the patients with multiple EEG NPA combinations, we assessed whether improvement in SWI (decrease of at least 25\%) and subjective improvement in daily functioning was associated with a change in slow wave slope course at $75 \mu \mathrm{V}$. Subsequently, a linear mixed model was fitted, incorporating all repeated measurements in the patients with multiple EEG NPA combinations. With this mixed model, we analysed possible correlation of overnight slow wave slope course at $75 \mu \mathrm{V}$ with total $I Q$, verbal $I Q$, performance IQ, processing speed, Beery test of visual-motor integration and digit span. The neuropsychological outcome measures were defined as the dependent variable, a random intercept per patient was used and the relative mean slow wave slope difference for the prespecified regions was included as a fixed variable. 
For statistically significant findings from linear regression analyses / linear mixed model analyses, we calculated Cohen's $f^{2}$ as an effect size measure as defined by using the formula Cohen's $f^{2}=R^{2} /\left(1-R^{2}\right)$. A Cohen's $f^{2} \geq 0.02$ is considered a small effect, $\geq 0.15$ a medium effect and $\geq 0.35$ a large effect.

The analyses were performed with MATLAB version 2018a and SPSS version 25. P-values $<0.05$ were considered significant. GraphPad Prism 8 was used for graphical illustration.

\section{Results}

\section{Patient characteristics and EEG / neuropsychological findings}

We included 29 patients. In total, 44 EEG NPA combinations were performed; 12 patients had two and 3 patients had three EEG $\sim$ NPA combinations that fulfilled the selection criteria. Patient characteristics are shown in table 1. The mean $( \pm \mathrm{sd})$ time between the EEG and NPA was $1.1 \pm 1.2$ months for the first, $0.5 \pm 1.3$ months for the second and $0.0 \pm 0.0$ months for the third EEG NPA combination, respectively. The median spike wave index was $90 \%$ for the first EEG, 91\% for the second EEG and 70\% for the third EEG. Concurrent treatment with conventional anti-epileptic drugs and corticosteroids was much more prevalent at the time of the second and third EEG as compared to the time of first EEG. EEG and neuropsychological findings are shown in table 2.

\section{Slow wave characteristics, overnight course and regional differences}

The mean $( \pm \mathrm{sd})$ amplitude of slow waves across all electrodes was $67 \pm 19 \mu \mathrm{V}$ in the $\mathrm{FH}$ and decreased to $41 \pm 14 \mu \mathrm{V}$ in the LH of non-REM sleep (-38\%). The mean absolute slope of slow waves decreased from $322 \pm 94$ in the $\mathrm{FH}$ to $187 \pm 67 \mu \mathrm{V} / \mathrm{s}$ in the $\mathrm{LH}(-41 \%)$. The mean normalized slope (at $75 \mu \mathrm{V}$, referred to as slow wave slope in the following analyses) over all electrodes decreased from $357 \pm 19$ to $327 \pm 22 \mu \mathrm{V} / \mathrm{s}(-8 \%, \mathrm{p}<0.001)$. The slow wave slope in the LH of non-REM sleep was significantly lower than during the FH of non-REM sleep for all electrode regions ( $p<0.001$ for all electrodes, focus electrodes, non-focus electrodes as well as DMN electrodes). The slow wave slope was higher in the focus than in the nonfocus electrodes, both in the first and the last hour (both $p<0.001$ ). The relative change in slow wave slope from first to last hour of non-REM sleep was different between the focus and the non-focus electrodes (-5.6\% for focus electrodes and $-8.7 \%$ for non-focus electrodes; $p=0.003)$. The overnight slow wave slope course is shown in figure 1. A detailed overview of overnight slow wave course for all regions of interest is provided in table S1. 
Table 1. patient characteristics $(n=29)$

\begin{tabular}{ll}
\hline male (\%) & $\mathbf{1 6}(\mathbf{5 5 \%})$ \\
\hline age at ESES diagnosis: mean \pm Sd & $75 \pm 26$ months / 6.3 \pm 2.2 years \\
presumed (main) aetiology: & \\
structural & $10(35 \%)$ \\
genetic / syndromal & $4(14 \%)$ \\
post-infectious & $1(3 \%)$ \\
unknown & $14(48 \%)$ \\
MRI abnormalities (incl. non-epileptogenic) & $19(66 \%)$ \\
lateralisation of MRI abnormalities: & \\
left & $3(10 \%)$ \\
right & $3(10 \%)$ \\
bilateral & $13(45 \%)$ \\
none & $10(35 \%)$ \\
frequency of seizures at time of diagnosis & \\
daily & $4(14 \%)$ \\
monthly & $10(35 \%)$ \\
yearly & $5(17 \%)$ \\
sporadic & $8(28 \%)$ \\
none & $2(7 \%)$ \\
development before ESES onset & \\
normal & $10(35 \%)$ \\
mild delay & $11(38 \%)$ \\
moderate delay & $4(14 \%)$ \\
severe delay & $4(14 \%)$ \\
\hline
\end{tabular}

\section{Overnight course of slow wave slope}

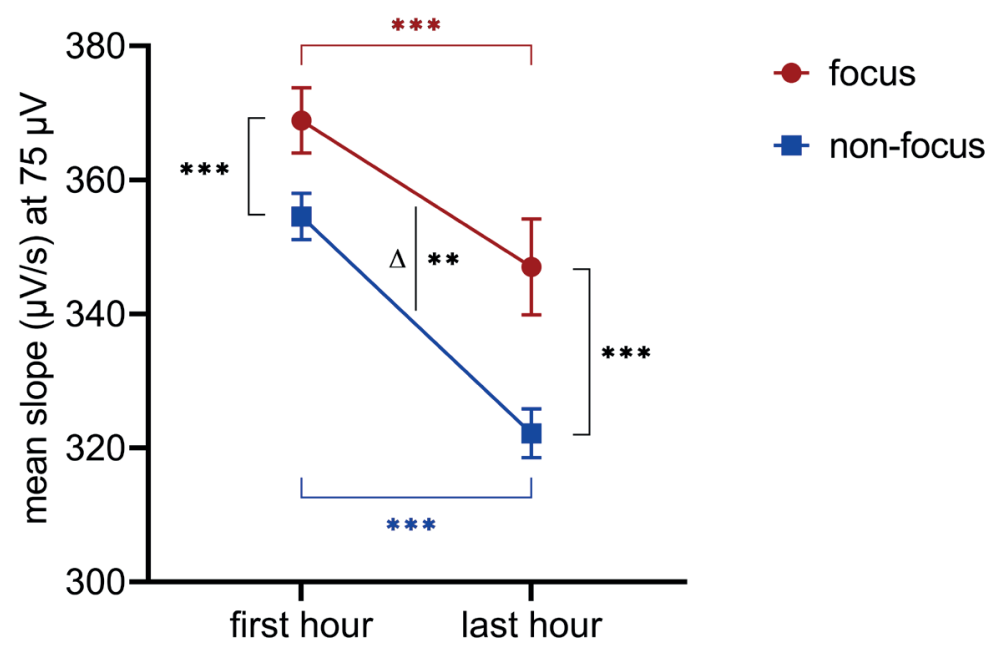

Figure 1. Overnight course of slow wave slope.

Differences were tested with dependent t-tests; ${ }^{* *} p<0.01$, *** $p<0.001$ 
Table 2. EEG and neuropsychological assessment findings $(n=44)$

\begin{tabular}{|c|c|c|c|}
\hline Combination of EEG NPA & first $(n=29)$ & second $(n=12)$ & third $(n=3)$ \\
\hline time between EEG and NPA (months): mean \pm sd & $1.1 \pm 1.2$ & $0.5 \pm 1.3$ & $0.0 \pm 0.0$ \\
\hline \multicolumn{4}{|l|}{ EEG findings whole night EEG } \\
\hline age at EEG recording (years): mean $\pm s d$ & $6.7 \pm 2.2$ & $7.5 \pm 1.7$ & $8.9 \pm 1.4$ \\
\hline time between diagnosis and EEG (months): mean \pm sd & $6 \pm 11$ & $12 \pm 12$ & $20 \pm 0.6$ \\
\hline SWI (\%): median (range) & $90(62-100)$ & $91(0-100)$ & $70(46-95)$ \\
\hline normal background pattern (\%) & $22(76 \%)$ & $10(83 \%)$ & $3(100 \%)$ \\
\hline sleep phenomena present $(\%)$ & $24(83 \%)$ & $11(92 \%)$ & $3(100 \%)$ \\
\hline \multicolumn{4}{|l|}{ Distribution of epileptiform activity in sleep: } \\
\hline generalised & $9(31 \%)$ & $4(33 \%)$ & $1(33 \%)$ \\
\hline multifocal bilateral & $8(28 \%)$ & $2(17 \%)$ & $1(33 \%)$ \\
\hline multifocal unilateral & $5(17 \%)$ & $0(0 \%)$ & $0(0 \%)$ \\
\hline focal unilateral & $7(24 \%)$ & $4(33 \%)$ & $1(33 \%)$ \\
\hline no epileptiform abnormalities & $0(0 \%)$ & $2(17 \%)$ & $0(0 \%)$ \\
\hline concurrent AED treatment & $19(66 \%)$ & $10(83 \%)$ & $2(67 \%)$ \\
\hline concurrent benzodiazepine treatment & $5(17 \%)$ & $6(50 \%)$ & $1(33 \%)$ \\
\hline concurrent corticosteroid treatment & $5(17 \%)$ & $7(58 \%)$ & $0(0 \%)$ \\
\hline \multicolumn{4}{|l|}{ Neuropsychological findings } \\
\hline Total IQ: mean \pm sd & $78 \pm 17(n=22)$ & $84 \pm 17(n=9)$ & $84 \pm 5(n=3)$ \\
\hline Verbal IQ: mean \pm sd & $84 \pm 15(n=18)$ & $87 \pm 15(n=8)$ & $88 \pm 8(n=3)$ \\
\hline Performance IQ: mean \pm sd & $84 \pm 18(n=19)$ & $84 \pm 19(n=9)$ & $83 \pm 5(n=3)$ \\
\hline $\mathrm{DQ}$ (if no IQ available): mean \pm sd & $43 \pm 37(n=4)$ & $29 \pm 10(n=2)$ & \\
\hline Processing speed: mean \pm sd & $75 \pm 15(n=13)$ & $80 \pm 21(n=9)$ & $90 \pm 6(n=2)$ \\
\hline Beery VMI: mean \pm sd & $89 \pm 11(n=19)$ & $85 \pm 10(n=9)$ & $87 \pm 6(n=3)$ \\
\hline Memory: Digit Span (WISC III): mean \pm Sd & $7 \pm 3(n=12)$ & $8 \pm 4(n=8)$ & $8 \pm 2(n=3)$ \\
\hline Peabody Picture Vocabulary Test: mean \pm sd & $105 \pm 18(n=10)$ & $95 \pm 22(n=7)$ & $91 \pm 6(n=3)$ \\
\hline Presence of behavioural problems (\%) & $12(41 \%)$ & $6(50 \%)$ & $0(0 \%)$ \\
\hline
\end{tabular}

Legend:

EEG: electroencephalogram, NPA: neuropsychological assessment, SWI: spike-wave index, IQ: intelligence quotient, DQ: developmental quotient, Beery VMI: Beery developmental test of visual-motor integration

WISC III: Wechsler Intelligence Scale for Children-III

\section{Relation between slow wave slope and SWI}

The mean slow wave slope at $75 \mu \mathrm{V}$ across all electrodes in the last hour of non-REM sleep was significantly correlated to SWI (rho 0.37; $p=0.049$ ), while slow wave slope in the first hour and slow wave slope change were not significantly correlated to SWI (rho 0.26; $p=0.17$ and rho 0.12; $p=0.53$ ). The slow wave slope of the last hour in focus electrodes showed a non-significant trend towards positive correlation with SWI (Rho 0.36, p=0.054), while the other slow wave characteristics for focus and non-focus electrodes were not significantly correlated to SWI (table S2). 


\section{Cross-sectional relation between overnight slope change, baseline patient characteristics, EEG findings and neuropsychological functioning at first combined investigation}

Among the clinical variables, the severity of developmental abnormalities before ESES onset was strongly associated with total IQ and verbal IQ ( $\beta-15.7, p=0.001$, Cohen's $f 2=0.71$ and $\beta-12.7, p=0.01$, Cohen's $f 2=0.47$ ) over the course of the disease (table 3 ). The percentage of time occupied by epileptiform discharges was also correlated to neuropsychological functioning: the higher the SWI, the lower total IQ and performance IQ ( $\beta-0.7, p=0.04$, Cohen's $\mathrm{f} 2=0.24$ and $\beta-0.8, p=0.01$, Cohen's $\mathrm{f} 2=0.44)$. The mean relative slope change (at $75 \mu \mathrm{V}$ ) across all electrodes, focus, non-focus and DMN electrodes was not related to the neuropsychological outcomes in this analysis (table 3).

Table 3. Univariate Linear regression analysis of clinical, EEG and NPA findings of first WN EEG $\sim$ NPA combination

\begin{tabular}{|c|c|c|c|c|c|c|}
\hline & $\begin{array}{c}\text { TIQ } \\
\beta \text { (p-value) }\end{array}$ & $\begin{array}{c}\text { VIQ } \\
\beta \text { (p-value) }\end{array}$ & $\begin{array}{c}\text { PIQ } \\
\beta \text { (p-value) }\end{array}$ & $\begin{array}{c}\text { PS } \\
\beta \text { (p-value) }\end{array}$ & $\begin{array}{c}\text { VMI } \\
\beta \text { (p-value) }\end{array}$ & $\begin{array}{c}\text { DS } \\
\beta \text { (p-value) }\end{array}$ \\
\hline MRI abnormalities & $1.8(0.81)$ & $-9.0(0.22)$ & $1.4(0.87)$ & $7.3(0.41)$ & $-7.9(0.11)$ & $\begin{aligned}-3.8(0.04) \\
f^{2}=0.55\end{aligned}$ \\
\hline Seizure frequency & $-0.5(0.88)$ & $-0.1(0.97)$ & $-1.0(0.77)$ & $1.8(0.63)$ & $-1.4(0.51)$ & $1.0(0.22)$ \\
\hline $\begin{array}{l}\text { Presence / severity of } \\
\text { developmental deficit before ESES }\end{array}$ & $\begin{array}{c}-15.7(0.001) \\
f^{2}=0.71\end{array}$ & $\begin{array}{c}-12.7(0.01) \\
f^{2}=0.47\end{array}$ & $-11.1(0.07)$ & $-11.6(0.18)$ & $-5.5(0.19)$ & $-1.4(0.39)$ \\
\hline SWI & $\begin{array}{c}-0.7(0.04) \\
f^{2}=0.24\end{array}$ & $-0.5(0.12)$ & $\begin{array}{c}-0.8(0.01) \\
f^{2}=0.44\end{array}$ & $-0.34(0.39)$ & $-0.37(0.09)$ & $0.06(0.57)$ \\
\hline sleep phenomena & $7.0(0.51)$ & $5.1(0.60)$ & $18.7(0.09)$ & $10.4(0.40)$ & $10.1(0.14)$ & $1.18(0.75)$ \\
\hline $\begin{array}{l}\text { all electrodes relative slope } \\
\text { change (at } 75 \mu \mathrm{V} \text { ) }\end{array}$ & $-14.1(0.84)$ & $51.4(0.81)$ & $-21.5(0.76)$ & $0.3(1.00)$ & $52.6(0.21)$ & $-0.02(0.99)$ \\
\hline $\begin{array}{l}\text { focus relative slope change } \\
\text { (at } 75 \mu \mathrm{V} \text { ) }\end{array}$ & $10.5(0.83)$ & $30.4(0.51)$ & $-29.2(0.55)$ & $-33.8(0.50)$ & $33.8(0.25)$ & $-0.01(0.99)$ \\
\hline $\begin{array}{l}\text { non-focus relative slope change } \\
\text { (at } 75 \mu \mathrm{V} \text { ) }\end{array}$ & $-19.3(0.78)$ & $55.0(0.42)$ & $-3.4(0.96)$ & $29.5(0.71)$ & $55.1(0.20)$ & $0.4(0.98)$ \\
\hline $\begin{array}{l}\text { DMN relative slope change } \\
\text { (at } 75 \mu \mathrm{V} \text { ) }\end{array}$ & $-19.1(0.80)$ & $48.9(0.52)$ & $-18.7(0.81)$ & $0.9(0.99)$ & $45.1(0.33)$ & $2.0(0.91)$ \\
\hline
\end{tabular}

Legend:

NPA: Neuropsychological assessment, TIQ: total intelligence quotient, VIQ: verbal intelligence quotient, PIQ: performal intelligence quotient, PS: processing speed VMI: Beery developmental test of visual-motor integration, DS: digit span, SWI: spike-wave index, DMN: default-mode network, ${ }^{2}$ : Cohen's f $^{2}$

\section{Cross-sectional relation between slow wave characteristics, SWI, neuropsychological test results and treatment with behaviour at first investigation}

Patients with behavioural problems showed a smaller decrease in slow wave slope (at 75 $\mu \mathrm{V})$ than those without, across all electrodes (-5.2\% vs $-10.2 \% ; \mathrm{p}=0.02)$, focus electrodes $(-0.9 \%$ vs $-8.8 \% ; p=0.006$, figure 2$)$, non-focus electrodes $(-6.1 \%$ vs $-10.6 \%, p=0.04)$ and 
DMN electrodes (-5.9\% vs $-11.3 \% ; p=0.02$ ) (table 4). The SWI was $84 \%$ for patients with and 90\% for patients without behavioural abnormalities and this difference was not significant $(p=0.20)$. Neuropsychological test results did not differ significantly between the patients with and those without behavioural problems (table S3). The presence or absence of treatment with AED, benzodiazepines or steroids at the time of the investigation was not associated with the presence or absence of behavioural problems (table S4).

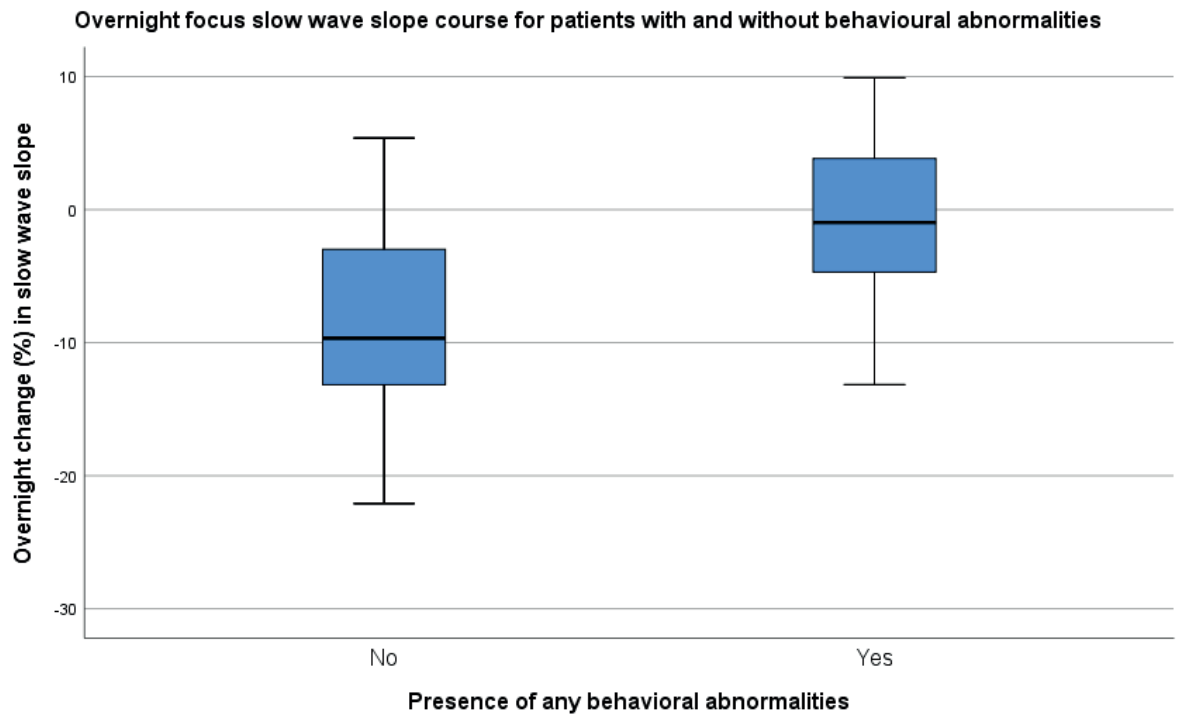

Figure 2. Overnight focus slow wave slope course for patients with and without behavioural abnormalities

Table 4. Slow wave characteristics for patients with and without behavioural problems (first EEG NPA combination)

\begin{tabular}{lccc}
\hline & behavioural problems ( $\mathbf{n}=\mathbf{1 7})$ & no behavioural problems ( $\mathbf{n}=12)$ & $\mathbf{p}$-value \\
\hline all electrodes relative slope change (at $75 \mu \mathrm{V})$ & $-5.2 \%$ & $-10.2 \%$ & 0.02 \\
focus relative slope change (at $75 \mu \mathrm{V})$ & $-0.9 \%$ & $-8.8 \%$ & 0.006 \\
non-focus relative slope change $($ at $75 \mu \mathrm{V})$ & $-6.1 \%$ & $-10.6 \%$ & 0.04 \\
DMN relative slope change (at $75 \mu \mathrm{V})$ & $-5.9 \%$ & $-11.3 \%$ & 0.02 \\
\hline
\end{tabular}

Legend:

EEG: electroencephalogram, NPA: Neuropsychological assessment, DMN: default-mode network 


\section{Longitudinal changes of slow wave slope characteristics versus changes in SWI and subjective daily functioning}

Of the 12 patients with at least two EEG NPA combinations, 3 showed SWI improvement and 9 showed subjective improvement in daily functioning between the first and the second time point. The difference in the overnight slow wave slope course (at $75 \mu \mathrm{V}$ ) between their first and second EEG NPA combination did not differ significantly for patients with SWI improvement versus those without SWI improvement; neither was subjective cognitive improvement associated with difference in overnight slow wave slope course (at $75 \mu \mathrm{V}$ ) (table 5).

The mixed model analysis of patients with multiple EEG NPA combinations showed no significant correlation of overnight slow wave slope course (at $75 \mu \mathrm{V}$ ) with $\mathrm{TIQ}, \mathrm{VIQ}, \mathrm{PIQ}$, PS, VMI or digit span (table S5).

Table 5. Slow wave characteristics for patients with and without improvement in SWI and daily functioning between first and second EEG NPA combination*

\begin{tabular}{|c|c|c|c|c|c|c|}
\hline & \multicolumn{3}{|c|}{ SWI improvement } & \multicolumn{3}{|c|}{ improvement daily functioning } \\
\hline & yes $(n=3)^{\#}$ & no $(n=9)$ & p-value & yes $(n=9)$ & no $(n=3)$ & p-value \\
\hline $\begin{array}{l}\text { all electrodes relative slope change } \\
\text { (at } 75 \mu \mathrm{V} \text { ) difference }\end{array}$ & $-1.2 \%$ & $+0.4 \%$ & 0.22 & $+2.2 \%$ & $+0.7 \%$ & 0.70 \\
\hline $\begin{array}{l}\text { focus relative slope change } \\
\text { (at } 75 \mu \mathrm{V} \text { ) difference }\end{array}$ & $+0.7 \%$ & $+1.9 \%$ & 0.79 & $+1.8 \%$ & $+1.0 \%$ & 0.86 \\
\hline $\begin{array}{l}\text { non-focus relative slope change } \\
(\text { at } 75 \mu \mathrm{V}) \sim \text { difference }\end{array}$ & $-2.6 \%$ & $+3.5 \%$ & 0.11 & $+2.0 \%$ & $+0.7 \%$ & 0.67 \\
\hline $\begin{array}{l}\text { DMN relative slope change } \\
(\text { at } 75 \mu \mathrm{V}) \text { difference }\end{array}$ & $-1.4 \%$ & $+3.5 \%$ & 0.29 & $+2.2 \%$ & $+2.5 \%$ & 0.95 \\
\hline
\end{tabular}

Legend:

* mean \pm sd interval between first and second EEG NPA combination: 7 \pm 3 (range 5-17) months

\#The 3 patients with > 25\% decrease in SWI had an SWI decrease from 75 to $8 \%$, from 68 to $0 \%$ and from 84 to $0 \%$ respectively.

NPA: Neuropsychological assessment, SWI: spike-wave index, DMN: default-mode network

\section{Discussion}

With this study of patients with epileptic encephalopathy with ESES, we found that: 1. the overnight slow wave slope decline is less pronounced in electrodes representing the epileptic focus as compared to the non-focus electrodes, hence slow wave homeostasis is more altered in the epileptic focus; 2 . in a cross-sectional comparison between patients, neurodevelopment, as measured with a neuropsychological assessment, does not relate to overnight slow wave slope course across patients. Patients with behavioural problems have a more impaired slow wave slope decline as compared to patients without reported 
or diagnosed behavioural problems, with the largest difference seen in the focus electrodes. 3. Longitudinal changes in slow wave slope course across the night were not associated with longitudinal changes in SWI and neuropsychological test results, or subjective improvement in daily functioning.

The difference in overnight slow wave slope course between focus electrodes and nonfocus electrodes that we found is in line with previous observations. In a study of 14 patients with ESES, the slow wave slope was not significantly different between focus and non-focus electrodes in the first hour of sleep, while in the last hour the non-focus electrodes showed a smaller slow wave slope. The authors found that the relative decline of slow wave slope was just not significantly different between the focus and non-focus electrodes ${ }^{31}$. Our study further assessed these parameters in a larger cohort of 29 patients and found that both first and last hour slow wave slope is higher in focus electrodes compared to non-focus electrodes and that the slow wave slope decline across the focus electrodes is less prominent as compared to the non-focus electrodes. These findings provide additional evidence that slow wave downscaling is locally impaired by epileptiform activity in children with epileptic encephalopathy with ESES and implicate that local synaptic homeostasis might be affected by spike waves.

In contrast to our expectations, we did not find a relation between overnight course of slow wave slope and neuropsychological test results. Previous studies have linked slow wave sleep to learning by showing that slow wave activity is increased locally at sleep onset after a visual learning task ${ }^{24}$ and that a reduced homeostatic decrease in slow wave slope is correlated with poorer daytime visuomotor learning ${ }^{26}$. Another study found that the normal overnight improvement in performance on a memory task was absent in patients with predominant epileptic activity during sleep and at least in one task related to spike wave density and suggested that this may reflect impaired sleep-related memory consolidation ${ }^{37}$. That we did not find a relation between overnight slow wave slope course and neuropsychological test results in our cross-sectional (between patients) analysis may be attributed to several aspects:

1. the context of an enormous variability in patient characteristics. The presence of structural lesions, pre-existing developmental deficits, the location of epileptiform abnormalities and treatment may explain most of the variability in test performance between the children with epileptic encephalopathy with ESES 11,38-40. This possible explanation is supported by the observation that in our linear regression analysis, developmental abnormalities before ESES are the strongest determinant of total and verbal IQ over the course of ESES. Previous studies showed that age at ESES diagnosis and ESES duration are important determinants of cognitive functioning; earlier onset and 
Chapter 5. Sleep slow waves and cognition in children with ESES

longer ESES duration are associated with worse cognitive outcome ${ }^{39,40}$. Also, previous studies have shown that slow wave activity shows an age-dependent evolution ${ }^{41,42}$. The variability of these factors may have complicated the assessment of the relation of overnight slow wave course and neuropsychological test results.

2. We suggest that the neuropsychological tests we used were relatively insensitive to fluctuations occurring in the context of ESES. A substantial part of the included patients had EEGs showing focal ESES (24\% of the first EEGs, 33\% of the second and third EEGs). Previous publications have suggested that standard neuropsychological assessment may be relatively insensitive to specific cognitive deficits in children with focal ESES ${ }^{43,44}$. Therefore, the conventional neuropsychological outcome measures that we analysed, may have been insensitive to deficits in the context of these patient. The insensitivity of neuropsychological assessment to fluctuations in the context of ESES, was also suggested by a previous study. In this study, we found that subjective improvement in cognitive functioning after treatment was not significantly related to TIQ improvement and that SWI decrease was associated with subjective cognitive improvement, while it was not related to TIQ change ${ }^{13}$. In our current study, we also included subjective improvement in cognitive functioning, as assessed by the paediatric neurologist during the outpatient clinic visits. Their assessment was based on information provided by the parents / guardians of the child. Often the information of the teachers was reported by the parents during the visits. Although this is a subjective outcome measure, we thought it could be of interest because subjective improvement can take any form and may therefore be more sensitive to changes with regard to the specific deficits in the individual child, in comparison to the neuropsychological assessment. Also, the subjective improvement may be clinically highly relevant. However, in our current analysis of overnight slow wave slope course versus subjective improvement in functioning in the subgroup of patients with multiple EEG $\sim$ NPA combinations, we did not find a relation either. This analysis, however, was based on a relatively small number of patients $(n=12)$.

Most of the abovementioned factors of influence on neurodevelopment should be of less influence in the mixed linear model analysis (thus correcting for the correlation between measurements from the same patient). However, also in this analysis we did not find a relation between slow wave slope course and neuropsychological test results and subjective cognitive functioning. We speculate that the relatively small sample size of patients with multiple EEG NPA combinations ( $n=12$ ) may have been insufficient to detect a relation or that change in treatment (e.g. benzodiazepine, corticosteroids) between the time points blurred a possible relation. Also, within the group with combined longitudinal EEG - NPA assessment, most patients still had a high SWI during the second and third follow-up (9 of 12 patients and 2 of 3 patients respectively). This suggests that these patients were still in the active phase of ESES and that overt neuropsychological disturbances could appear later. 
Interestingly, we did find a relation between behavioural problems and slow wave slope course across the night. For all combinations of electrodes (average of all electrodes, focus, non-focus, and DMN electrodes), the slow wave slope showed a smaller decline for patients with behavioural problems compared to patients without reported or diagnosed behavioural problems and this difference was most pronounced for the focus electrodes. We hypothesize that behaviour of patients with ESES, as suggested by a previous study ${ }^{4}$, fluctuates easily and therefore is more closely related to the slow wave homeostasis at a certain moment in time. A strong contribution of treatment to these fluctuations could be suggested, however we did not find any association of AED, benzodiazepine or corticosteroid treatment with the presence or absence of behavioural problems. To our knowledge, this concept has not been investigated in previous studies.

Our results need to be interpreted carefully. Firstly, the heterogeneity of our study population, including patients with newly diagnosed as well as longstanding ESES, with and without structural aetiology and with and without treatment, increases variability and hence confounding factors limit the generalisability of our findings. However, the heterogeneity of our cohort is representative for the broad spectrum of patients with ESES 1,11. Secondly, although we have used different approaches that all did not yield a clear relation between slow wave characteristics and neuropsychological test results, our study was not designed (and underpowered) to prove that there is no relation. Furthermore, the design of our study, relating the overnight course of slow wave slope to neuropsychological test results of one test session might have been insufficient to analyse the effect of ESES on synaptic homeostasis. Testing overnight performance improvement (i.e. comparison of test results before and after a night's sleep) may be a better method to answer this research question.

In conclusion, we add evidence that, in children with ESES, sleep slow wave homeostasis is altered by epileptic activity as it is most affected in the epileptic focus. The presence of behavioural problems is associated with reduced slow wave downscaling, while we did not find a relation between overnight slow wave slope decline and neuropsychological test results. Based on the previously reported relationship between slow waves and network synchrony and indirectly synaptic strength, we might conclude that synaptic homeostasis is disturbed by epileptiform activity in children with ESES, at most in the epileptic focus; future studies with overnight EEG recordings and overnight neuropsychological testing with appropriate tests (i.e. testing before a night's sleep and repeat testing afterwards), may elucidate whether this disturbance of synaptic homeostasis explains the occurrence of cognitive deficits. 


\section{Acknowledgements}

We thank Monique van Schooneveld, paediatric neuropsychologist, for her advice regarding the design of the study and the interpretation of neuropsychological findings.

We thank Eltje Bloemen, research nurse, for her involvement in the planning of whole night EEGs, neuropsychological assessments and her general support and approachability for the ESES patients.

\section{Disclosure statements}

- This study was funded by the Dutch Epilepsy Fund (Epilepsiefonds, grant 13-17) and the Wilhelmina Children's Hospital Research Fund (grant R2634). The funding sources had no direct involvement in the conduct of the study.

- Non-financial disclosure: None. 


\section{References}

1. Sánchez Fernández I, Loddenkemper T, Peters JM, Kothare S V. Electrical status epilepticus in sleep: Clinical presentation and pathophysiology. Pediatr Neurol. 2012;47(6):390-410. doi:10.1016/j. pediatrneurol.2012.06.016

2. Nickels K, Wirrell E. Electrical Status Epilepticus in Sleep. Semin Pediatr Neurol. 2008;15(2):50-60. doi:10.1016/j.spen.2008.03.002

3. Patry G, Lyagoubi S, Tassinari CA. Subclinical "electrical status epilepticus"induced by sleep in children. Arch Neurol. 1971;24(3):242-252. doi:http://dx.doi.org/10.1001/archneur.1971.00480330070006

4. Lesca G, Rudolf G, Bruneau N, et al. GRIN2A mutations in acquired epileptic aphasia and related childhood focal epilepsies and encephalopathies with speech and language dysfunction. Nat Genet. 2013;45(9):1061-1066. doi:10.1038/ng.2726

5. Carvill GL, Regan BM, Yendle SC, et al. GRIN2A mutations cause epilepsy-aphasia spectrum disorders. Nat Genet. 2013;45(9):1073-1076. doi:10.1038/ng.2727

6. Kersbergen KJ, De Vries LS, Leijten FSS, et al. Neonatal thalamic hemorrhage is strongly associated with electrical status epilepticus in slow wave sleep. Epilepsia. 2013;54(4):733-740. doi:10.1111/ epi.12131

7. Guzzetta F, Battaglia D, Veredice C, et al. Early thalamic injury associated with epilepsy and continuous spike-wave during slow sleep. Epilepsia. 2005;46(6):889-900. doi:10.1111/j.1528-1167.2005.64504.x

8. Lemke JR, Lal D, Reinthaler EM, et al. Mutations in GRIN2A cause idiopathic focal epilepsy with rolandic spikes. Nat Genet. 2013;45(9):1067-1072. doi:10.1038/ng.2728

9. Scheltens-De Boer M. Guidelines for EEG in encephalopathy related to ESES/CSWS in children. In: Epilepsia. Vol 50. ; 2009:13-17. doi:10.1111/j.1528-1167.2009.02211

10. Nissenkorn A, Pappo A, Feldmann Y, et al. Original article Influence of epileptic activity during sleep on cognitive performance in benign childhood epilepsy with centrotemporal spikes. Eur J Paediatr Neurol. 2017:1-6. doi:10.1016/j.ejpn.2017.07.001

11. Van Hirtum-Das M, Licht EA, Koh S, Wu JY, Donald Shields W, Sankar R. Children with ESES: Variability in the syndrome. Epilepsy Res. 2006;70(SUPPL.1):248-258. doi:10.1016/j.eplepsyres.2006.01.020

12. van den Munckhof B, van Dee V, Sagi L, et al. Treatment of electrical status epilepticus in sleep: A pooled analysis of 575 cases. Epilepsia. 2015;56(11):1738-1746. doi:10.1111/epi.13128

13. van den Munckhof B, Alderweireld C, Davelaar S, et al. Treatment of electrical status epilepticus in sleep: Clinical and EEG characteristics and response to 147 treatments in 47 patients. Eur $\mathrm{J}$ Paediatr Neurol. 2018;22(1):64-71. doi:10.1016/j.ejpn.2017.08.006

14. Tononi G, Cirelli C. Sleep and synaptic homeostasis: A hypothesis. Brain Res Bull. 2003;62(2):143-150. doi:10.1016/j.brainresbull.2003.09.004

15. Tononi G, Cirelli C. Sleep function and synaptic homeostasis. Sleep Med Rev. 2006;10(1):49-62. doi:10.1016/j.smrv.2005.05.002

16. Tononi G, Cirelli C. Sleep and the Price of Plasticity: From Synaptic and Cellular Homeostasis to Memory Consolidation and Integration. Neuron. 2014;81 (1):12-34. doi:10.1016/j.neuron.2013.12.025

17. De Vivo L, Bellesi M, Marshall W, et al. Ultrastructural evidence for synaptic scaling across the wake/ sleep cycle. Science (80-). 2017;355(6324):507-510. doi:10.1126/science.aah5982

18. Vyazovskiy V V, Riedner B a, Cirelli C, Tononi G. Sleep homeostasis and cortical synchronization: II. A local field potential study of sleep slow waves in the rat. Sleep. 2007;30(12):1631-1642. doi:18246974

19. Riedner B a, Vyazovskiy VV, Huber R, et al. Sleep homeostasis and cortical synchronization: III. A highdensity EEG study of sleep slow waves in humans. Sleep. 2007;30(12):1643-1657. doi:18246974

20. Massimini M. The Sleep Slow Oscillation as a Traveling Wave. J Neurosci. 2004;24(31):6862-6870. doi:10.1523/JNEUROSCI.1318-04.2004

21. Steriade M, Timofeev I, Grenier F. Natural Waking and Sleep States: A View From Inside Neocortical Neurons. J Neurophysiol. 2001;85(5):1969-1985. doi:10.1152/jn.2001.85.5.1969 
Chapter 5. Sleep slow waves and cognition in children with ESES

22. Vyazovskiy $V$ V., Olcese U, Lazimy YM, et al. Cortical Firing and Sleep Homeostasis. Neuron. 2009;63(6):865-878. doi:10.1016/j.neuron.2009.08.024

23. Esser SK, Hill SL, Tononi G. Sleep homeostasis and cortical synchronization: I. Modeling the effects of synaptic strength on sleep slow waves. Sleep. 2007;30(12):1617-1630.

24. Huber R, Felice Ghilardi M, Massimini M, Tononi G. Local sleep and learning. Nature. 2004;430(6995):7881. doi:10.1038/nature02663

25. Ngo HVV., MartinetzT, Born J, Mölle M. Auditory closed-loop stimulation of the sleep slow oscillation enhances memory. Neuron. 2013. doi:10.1016/j.neuron.2013.03.006

26. Boly M, Jones B, Findlay $\mathrm{G}$, et al. Altered sleep homeostasis correlates with cognitive impairment in patients with focal epilepsy. Brain. 2017;140(4):1026-1040. doi:10.1093/brain/awx017

27. Holmes GL, Lenck-Santini PP. Role of interictal epileptiform abnormalities in cognitive impairment. Epilepsy Behav. 2006;8(3):504-515. doi:10.1016/j.yebeh.2005.11.014

28. Tassinari CA, Rubboli G. Cognition and paroxysmal EEG activities: From a single spike to electrical status epilepticus during sleep. Epilepsia. 2006;47(SUPPL. 2):40-43. doi:10.1111/j.15281167.2006.00686.x

29. Rubboli G, Huber R, Tononi G, Tassinari CA. Encephalopathy related to Status Epilepticus during slow Sleep: a link with sleep homeostasis? Epileptic Disord. 2019. doi:10.1684/epd.2019.1059

30. Bölsterli BK, Schmitt B, Bast T, et al. Impaired slow wave sleep downscaling in encephalopathy with status epilepticus during sleep (ESES). Clin Neurophysiol. 2011;122(9):1779-1787. doi:10.1016/j. clinph.2011.01.053

31. Bölsterli Heinzle BK, Fattinger S, Kurth S, et al. Spike wave location and density disturb sleep slow waves in patients with CSWS (continuous spike waves during sleep). Epilepsia. 2014;55(4):584-591. doi:10.1111/epi.12576

32. Bölsterli BK, Gardella E, Pavlidis E, et al. Remission of encephalopathy with status epilepticus (ESES) during sleep renormalizes regulation of slow wave sleep. Epilepsia. 2017;58(11):1892-1901. doi:10.1111/epi.13910

33. Massa R, de Saint-Martin a, Carcangiu R, et al. EEG criteria predictive of complicated evolution in idiopathic rolandic epilepsy. Neurology. 2001;57(6):1071-1079. http://www.ncbi.nlm.nih.gov/ pubmed/11571336.

34. Siniatchkin M, Groening K, Moehring J, et al. Neuronal networks in children with continuous spikes and waves during slow sleep. Brain. 2010;133(9):2798-2813. doi:10.1093/brain/awq183

35. Japaridze N, Muthuraman M, Dierck C, et al. Neuronal networks in epileptic encephalopathies with CSWS. Epilepsia. 2016;57(8):1245-1255. doi:10.1111/epi.13428

36. Van de Steen F, Almgren H, Razi A, Friston K, Marinazzo D. Dynamic causal modelling of fluctuating connectivity in resting-state EEG. Neuroimage. 2019;189:476-484. doi:10.1016/j. neuroimage.2019.01.055

37. Galer S, Urbain C, De Tiège $X$, et al. Impaired sleep-related consolidation of declarative memories in idiopathic focal epilepsies of childhood. Epilepsy Behav. 2015;43:16-23. doi:10.1016/j. yebeh.2014.11.032

38. Caraballo RH, Fortini S, Flesler S, Pasteris MC, Caramuta L, Portuondo E. Encephalopathy with status epilepticus during sleep: Unusual EEG patterns. Seizure. 2015. doi:10.1016/j.seizure.2014.10.003

39. Maltoni L, Posar A, Parmeggiani A. Long-term follow-up of cognitive functions in patients with continuous spike-waves during sleep (CSWS). Epilepsy Behav. 2016;60:211-217. doi:10.1016/j. yebeh.2016.04.006

40. Pera MC, Brazzo D, Altieri N, Balottin U, Veggiotti P. Long-term evolution of neuropsychological competences in encephalopathy with status epilepticus during sleep: A variable prognosis. Epilepsia. 2013;54(SUPPL.7):77-85. doi:10.1111/epi.12313 
41. Campbell IG, Feinberg I. Longitudinal trajectories of non-rapid eye movement delta and theta EEG as indicators of adolescent brain maturation. Proc Natl Acad Sci U S A. 2009;106(13):5177-5180. doi:10.1073/pnas.0812947106

42. Feinberg I, March JD, Flach K, Maloney T, Chern WJ, Travis F. Maturational changes in amplitude, incidence and cyclic pattern of the 0 to $3 \mathrm{~Hz}$ (delta) electroencephalogram of human sleep. Brain Dysfunct. 1990.

43. Kuki I, Kawawaki H, Okazaki S, Ikeda H, Tomiwa K. Epileptic encephalopathy with continuous spikes and waves in the occipito-temporal region during slow-wave sleep in two patients with acquired Kanji dysgraphia. Epileptic Disord. 2014. doi:10.1684/epd.2014.0698

44. Tassinari CA, Cantalupo G, Rubboli G. Focal ESES as a selective focal brain dysfunction: A challenge for clinicians, an opportunity for cognitive neuroscientists. Epileptic Disord. 2015. doi:10.1684/ epd.2015.0760

45. Seegmüller C, Deonna T, Mayor Dubois C, et al. Long-term outcome after cognitive and behavioral regression in nonlesional epilepsy with continuous spike-waves during slow-wave sleep. Epilepsia. 2012;53(6):1067-1076. doi:10.1111/j.1528-1167.2012.03465 


\section{Supplementary material}

Table S1: Mean slow wave characteristics of first whole-night EEG $(n=29)$

\begin{tabular}{|c|c|c|c|c|}
\hline electrode set & slow wave characteristic & first hour & last hour & change $(\%)$ \\
\hline \multirow{3}{*}{ all } & slow wave amplitude $(\mu \mathrm{V})$ : mean \pm sd & $67 \pm 19$ & $41 \pm 14$ & $-38 \pm 15$ \\
\hline & slow wave slope $(\mu \mathrm{V} / \mathrm{s})$ : mean \pm sd & $322 \pm 94$ & $187 \pm 67$ & $-41 \pm 16$ \\
\hline & slow wave slope at $75 \mu \mathrm{V}$ : mean \pm sd & $357 \pm 19$ & $327 \pm 22$ & $-8 \pm 6$ \\
\hline \multirow{3}{*}{ focus } & slow wave amplitude $(\mu \mathrm{V})$ : mean \pm sd & $73 \pm 24$ & $49 \pm 25$ & $-33 \pm 20$ \\
\hline & slow wave slope $(\mu \mathrm{V} / \mathrm{s})$ : mean \pm sd & $361 \pm 123$ & $234 \pm 131$ & $-35 \pm 21$ \\
\hline & slow wave slope at $75 \mu \mathrm{V}$ : mean \pm sd & $369 \pm 26$ & $347 \pm 39$ & $-6 \pm 8$ \\
\hline \multirow{3}{*}{ non-focus } & slow wave amplitude $(\mu \mathrm{V})$ : mean \pm sd & $66 \pm 18$ & $39 \pm 12$ & $-39 \pm 15$ \\
\hline & slow wave slope $(\mu \mathrm{V} / \mathrm{s})$ : mean \pm sd & $313 \pm 91$ & $177 \pm 58$ & $-42 \pm 16$ \\
\hline & slow wave slope at $75 \mu \mathrm{V}$ : mean \pm sd & $355 \pm 19$ & $322 \pm 20$ & $-9 \pm 6$ \\
\hline \multirow{3}{*}{ default-mode network } & slow wave amplitude $(\mu \mathrm{V})$ : mean \pm sd & $72 \pm 22$ & $46 \pm 24$ & $-37 \pm 19$ \\
\hline & slow wave slope $(\mu \mathrm{V} / \mathrm{s})$ : mean \pm sd & $351 \pm 116$ & $212 \pm 118$ & $-40 \pm 19$ \\
\hline & slow wave slope at $75 \mu \mathrm{V}$ : mean \pm sd & $362 \pm 23$ & $328 \pm 26$ & $-9 \pm 6$ \\
\hline
\end{tabular}

Legend:

first hour $=$ first hour of nREM sleep, last hour $=$ last hour of nREM sleep

Table S2: Correlation of mean slow wave characteristics and SWI of first WN EEG (n=29)

\begin{tabular}{llc}
\hline slow wave characteristic & Rho & p-value \\
\hline slow wave slope FH at $75 \mu \mathrm{V}$ across all electrodes & 0.26 & 0.17 \\
slow wave slope $L H$ at $75 \mu \mathrm{V}$ across all electrodes & $\mathbf{0 . 3 7}$ & $\mathbf{0 . 0 4 9}$ \\
relative slow wave slope change at $75 \mu \mathrm{V}$ across all electrodes & 0.12 & 0.53 \\
slow wave slope FH at $75 \mu \mathrm{V}$ across focus electrodes & 0.29 & 0.12 \\
slow wave slope $\mathrm{LH}$ at $75 \mu \mathrm{V}$ across focus electrodes & 0.36 & 0.054 \\
relative slow wave slope change at $75 \mu \mathrm{V}$ across focus electrodes & 0.26 & 0.17 \\
slow wave slope FH at $75 \mu \mathrm{V}$ across non-focus electrodes & 0.22 & 0.25 \\
slow wave slope LH at $75 \mu \mathrm{V}$ across non-focus electrodes & 0.28 & 0.14 \\
relative slow wave slope change at $75 \mu \mathrm{V}$ across non-focus electrodes & 0.14 & 0.48 \\
\hline
\end{tabular}

Legend:

$F H=$ first hour of nREM sleep, $L H=$ last hour of nREM sleep, Rho = Spearman's Rho 
Table S3: Neuropsychological assessment findings for patients with and without behavioural problems (first EEG NPA combination)

\begin{tabular}{lccc}
\hline & behavioural problems & no behavioural problems & p-value \\
\hline TIQ & $80(n=8)$ & $76(n=14)$ & 0.64 \\
VIQ & $89(n=8)$ & $80(n=10)$ & 0.22 \\
PIQ & $80(n=9)$ & $87(n=10)$ & 0.40 \\
PS & $68(n=5)$ & $79(n=8)$ & 0.19 \\
VMI & $89(n=9)$ & $88(n=10)$ & 0.77 \\
DS & $6(n=4)$ & $8(n=8)$ & 0.36 \\
\hline
\end{tabular}

Legend:

NPA: Neuropsychological assessment, TIQ: total intelligence quotient, VIQ: verbal intelligence quotient, PIQ: performal intelligence quotient, PS: processing speed, VMI: Beery developmental test of visual-motor integration , DS: digit span

Table S4: Treatment vs behavioural abnormalities (first EEG NPA combination, $\mathbf{n = 2 9}$ )

\begin{tabular}{|c|c|c|c|}
\hline & \multicolumn{2}{|c|}{ behavioural problems } & \multirow[b]{2}{*}{ Fisher's exact test for difference in proportion per treatment category } \\
\hline & yes & no & \\
\hline AED treatment & & & $p=1.0$ \\
\hline yes & $8(42 \%)$ & $11(58 \%)$ & \\
\hline no & $4(40 \%)$ & $6(60 \%)$ & \\
\hline benzodiazepine treatment & & & $p=1.0$ \\
\hline yes & $2(40 \%)$ & $3(60 \%)$ & \\
\hline no & $10(42 \%)$ & $14(58 \%)$ & \\
\hline corticosteroid treatment & & & $p=0.37$ \\
\hline yes & $1(20 \%)$ & $4(80 \%)$ & \\
\hline no & $11(46 \%)$ & $13(54 \%)$ & \\
\hline
\end{tabular}

Legend:

AED treatment : treatment with conventional anti-epileptic drugs

Table S5: Mixed linear model analysis of patients with multiple EEG NPA combinations

\begin{tabular}{|c|c|c|c|c|c|c|}
\hline & $\begin{array}{c}\text { TIQ } \\
\text { coeff } \\
\text { (p-value) }\end{array}$ & $\begin{array}{c}\text { VIQ } \\
\text { coeff } \\
\text { (p-value) }\end{array}$ & $\begin{array}{c}\text { PIQ } \\
\text { coeff } \\
\text { (p-value) }\end{array}$ & $\begin{array}{c}\text { PS } \\
\text { coeff } \\
\text { (p-value) }\end{array}$ & $\begin{array}{c}\text { VMI } \\
\text { coeff } \\
\text { (p-value) }\end{array}$ & $\begin{array}{c}\text { DS } \\
\text { coeff } \\
\text { (p-value) }\end{array}$ \\
\hline $\begin{array}{l}\text { all electrodes relative slope } \\
\text { change (at } 75 \mu \mathrm{V} \text { ) }\end{array}$ & $46.1(0.22)$ & $31.4(0.51)$ & $-32.5(0.45)$ & $56.0(0.46)$ & $30.5(0.36)$ & $-5.9(0.69)$ \\
\hline $\begin{array}{l}\text { focus relative slope change } \\
\text { (at } 75 \mu \mathrm{V} \text { ) }\end{array}$ & $18.3(0.57)$ & $29.4(0.41)$ & $-29.8(0.40)$ & $-24.6(0.65)$ & $42.5(0.09)$ & $4.4(0.69)$ \\
\hline $\begin{array}{l}\text { non-focus relative slope change } \\
\text { (at } 75 \mu \mathrm{V} \text { ) }\end{array}$ & $43.4(0.22)$ & $23.4(0.61)$ & $-29.6(0.48)$ & $84.7(0.25)$ & $22.3(0.49)$ & $-8.5(0.56)$ \\
\hline $\begin{array}{l}\text { DMN relative slope change } \\
\text { (at } 75 \mu \mathrm{V} \text { ) }\end{array}$ & $10.3(0.77)$ & $-9.1(0.82)$ & $-32.5(0.37)$ & $-14.1(0.84)$ & $19.53(0.53)$ & $0.29(0.98)$ \\
\hline
\end{tabular}




\section{PART II. \\ Treatment}

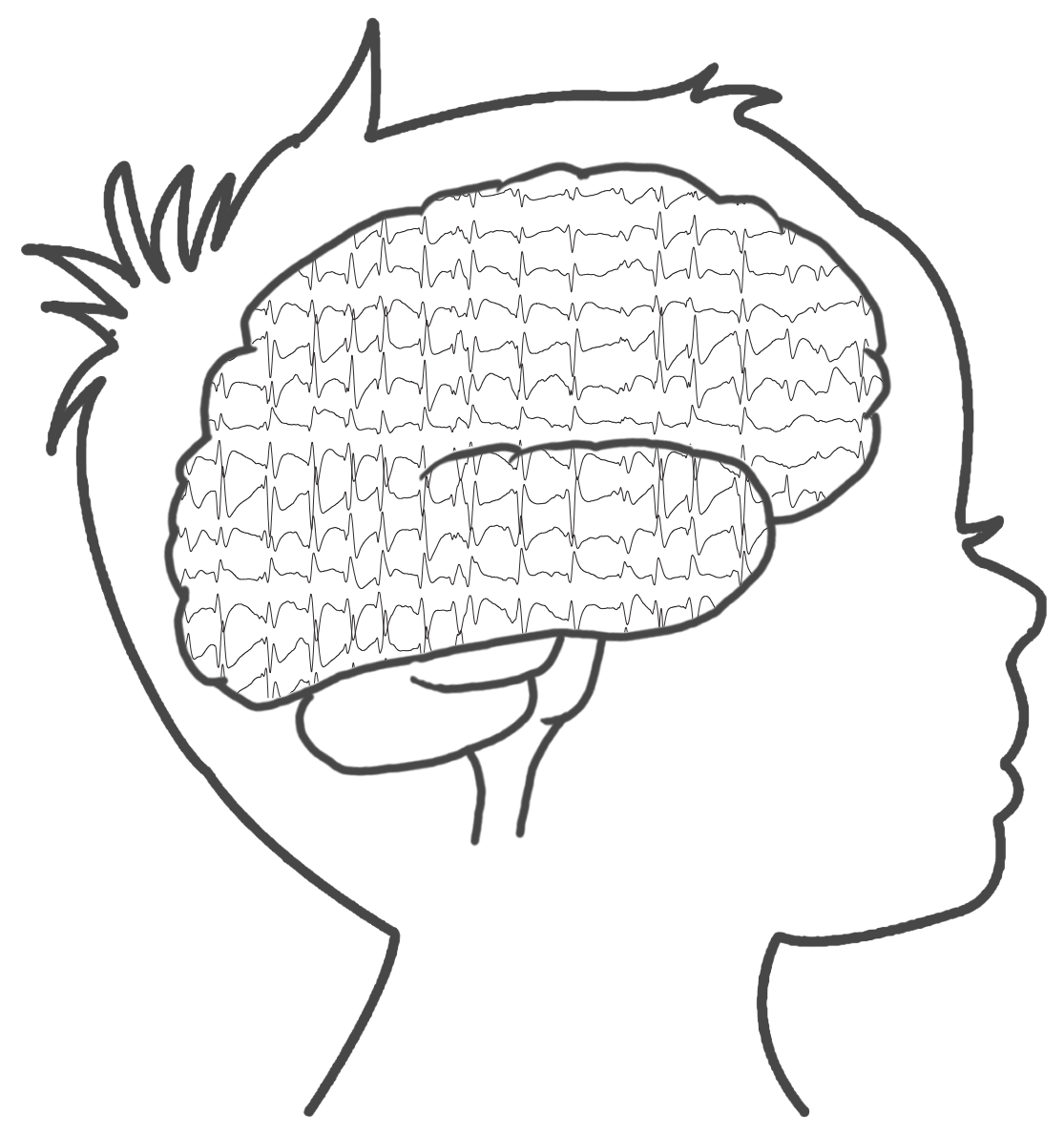



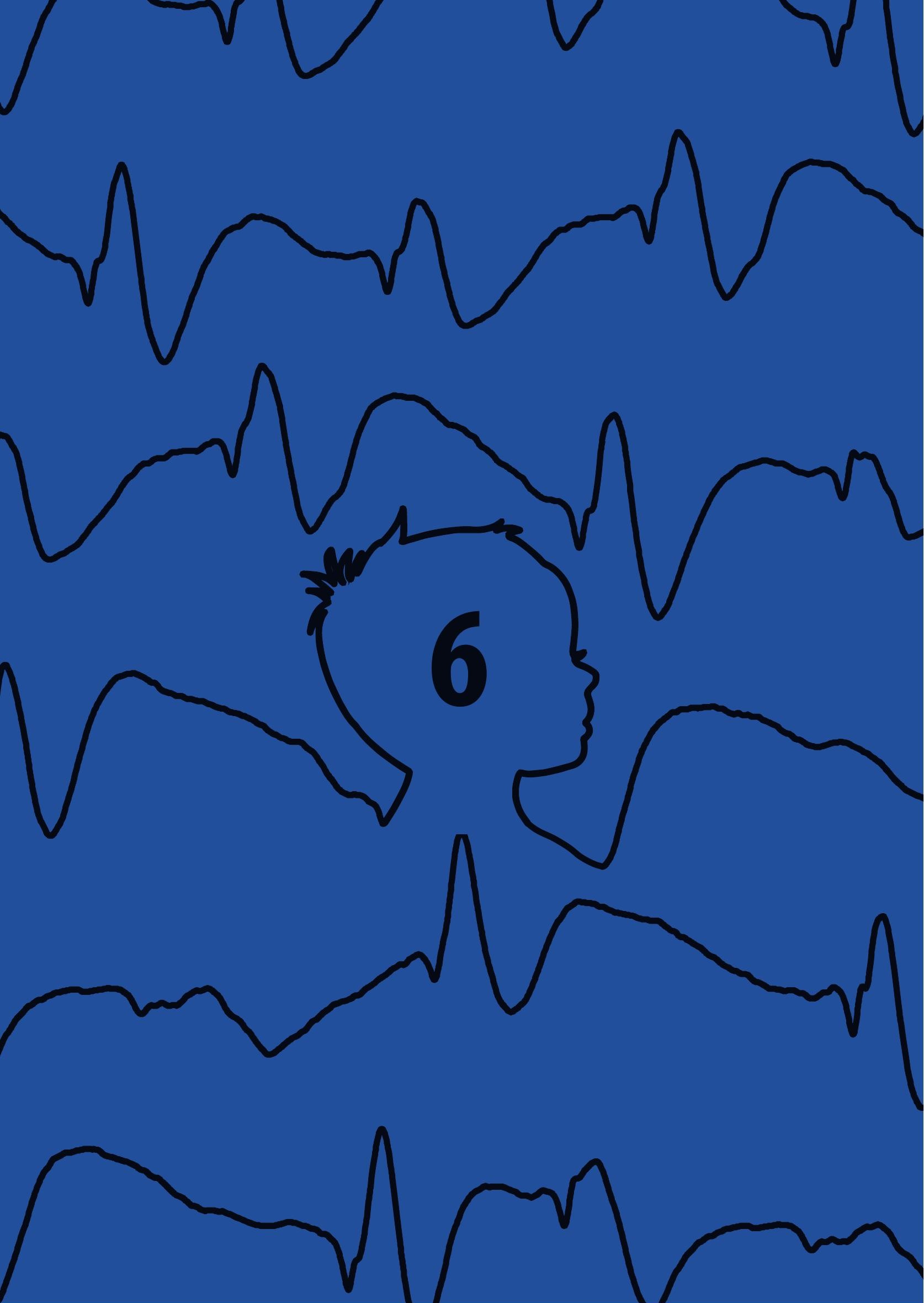


\section{Chapter 6}

\section{Treatment of Electrical Status Epilepticus in Sleep (ESES): a pooled analysis of 575 cases}

Bart van den Munckhof"

Violet van Dee*

Liora Sagi

Roberto H Caraballo

PierangeloVeggiotti

Elina Liukkonen

\author{
Tobias Loddenkemper \\ Iván Sánchez Fernández \\ Marga Buzatu \\ Christine Bulteau \\ Kees PJ Braun \\ Floor E Jansen
}

* These authors contributed equally to the manuscript. 


\section{Summary}

Objective: Epileptic encephalopathy with electrical status epilepticus in sleep (ESES) is a pediatric epilepsy syndrome with sleep-induced epileptic discharges and acquired impairment of cognition or behavior. Treatment of ESES is assumed to improve cognitive outcome. The aim of this study is to create an overview of the current evidence for different treatment regimens in children with ESES syndrome.

Methods: A literature search using Pubmed and Embase was performed. Articles were selected that contain original treatment data of patients with ESES syndrome. Authors were contacted for additional information. Individual patient-data were collected, coded and analyzed using logistic regression analysis. The three predefined main outcome measures were improvement in cognitive function, EEG pattern and any improvement (cognition or EEG).

Results: The literature search yielded 1766 articles. After applying in- and exclusion criteria, 112 articles and 950 treatments in 575 patients could be analyzed. Anti-epileptic drugs (AEDs, $n=495$ ) were associated with improvement (i.e. cognition or EEG) in 49\% of patients, benzodiazepines $(n=171)$ in $68 \%$ and steroids $(n=166)$ in $81 \%$. Surgery $(n=62)$ resulted in improvement in $90 \%$ of patients. In a subgroup analysis of patients who were consecutively reported (585 treatments in 282 patients), we found improvement in a smaller proportion treated with AEDs (34\%), benzodiazepines (59\%) and steroids (75\%), while the improvement percentage after surgery was preserved (93\%). Possible predictors of improved outcome were treatment category, normal development before ESES onset and the absence of structural abnormalities.

Significance: Although most included studies were small and retrospective and their heterogeneity allowed analysis of only qualitative outcome data, this pooled analysis suggests superior efficacy of steroids and surgery in encephalopathy with ESES.

\section{Key point box}

- Treatment of ESES syndrome is assumed to improve cognitive outcome.

- This pooled analysis of 575 cases suggests that corticosteroids and surgery are most effective, while benzodiazepines may be an appropriate alternative.

- Conventional anti-epileptic drugs were reported less effective.

- Evidence is limited to mostly retrospective case-series.

- A Randomized Controlled Trial is needed to provide definite answers for children with this often devastating epilepsy syndrome. 


\section{Introduction}

Electrical status epilepticus in sleep (ESES) was first described in 1971 in six children with continuous spike-wave discharges persisting during whole night's non-rapid eye movement (NREM) sleep and subsiding upon awakening. In the initial definition epileptic activity had to be present during at least $85 \%$ of the NREM sleep EEG, recorded on at least three occasions over a period of at least one month. More recently ESES cases with a spike-wave index of $50-85 \%$ were added to the spectrum. ${ }^{1-4}$

The clinical presentation of children with an ESES EEG pattern is variable. The most severe clinical syndrome can present with global cognitive regression in addition to clinical seizures. However, atypical cases have been described with developmental delay from birth or developmental arrest but no regression of cognitive functioning. The age at onset ranges from one to 14 years, with a peak between four and eight years. Although seizures may be absent in up to $20 \%$ of cases, they are most often the presenting symptom, after which a developmental delay, a developmental arrest or a regression in cognitive performance or behavior becomes evident. The Landau Kleffner Syndrome is closely related to ESES and is characterized by an acquired aphasia. In these patients the cognitive symptoms mainly focus on the language domain. These clinical syndromes are referred to as "epileptic encephalopathy with ESES", ESES syndrome or continuous spikes and waves during sleep (CSWS) syndrome. The EEG features of ESES frequently resolve spontaneously during puberty, while cognitive sequelae often remain. ${ }^{5-7}$

The concept of ESES was introduced describing patients with unknown etiology and has been expanded to patients with structural or genetic abnormalities. Recently, GRIN2A mutations were found to be present in up to $20 \%$, mostly the familiar cases. ${ }^{8-10}$ However, the etiology remains undetermined in the majority of patients. ${ }^{11-13}$ An underlying inflammatory process has been suggested, mainly because steroids appear to be effective in the treatment of ESES syndrome. Inflammation may be either the cause of ESES or an epiphenomenon. ${ }^{4,5,14,15}$ The mechanism of how continuous epileptic activity during sleep leads to cognitive decline is incompletely understood.

Although the importance of (early) treatment of convulsive status epilepticus has convincingly been demonstrated ${ }^{16}$, it remains to be established whether treatment of sleep-induced status epilepticus, the neurophysiological hallmark of ESES syndrome, prevents permanent cognitive impairment. Treatment decisions are often based on expert-opinion. Mainly small and retrospective studies have reported on the EEG effects or cognitive outcome of various conventional anti-epileptic drugs, benzodiazepines, steroids, intravenous immunoglobulins, ketogenic diet and epilepsy surgery. So far only one small 
randomized controlled trial has been performed, comparing levetiracetam with placebo in patients with ESES.17, 18 The aim of this meta analysis of individual patient data is to create an overview of all published treatments in children with epileptic encephalopathy with ESES and their effects on cognitive outcome or EEG pattern.

\section{Methods}

\section{Search strategy}

A literature search in PubMed and Embase was performed with various synonyms for ESES and treatment (table e-1). The initial search was performed in July 2012 yielding 1663 articles. An update on the 15th of May 2013 resulted in a total of 1766 articles. The search strategy was defined by three investigators ( $V v D$, BvdM, FEJ). The search was performed by two authors ( $V v D$ and $B v d M)$ and in rare cases of discordant judgment a third author was consulted (FEJ).

\section{Study selection and data-acquisition}

Published studies were selected for possible inclusion if the effect of treatment on the EEG pattern or on cognitive functioning was described. All search results were reviewed based on title and abstract by applying the in- and exclusion criteria (Figure 1). Of the selected articles full texts were reviewed. A cross reference check was performed to prevent missing relevant articles.

The corresponding authors of all selected studies were contacted for additional individual patient information including patient characteristics, qualitative and if possible quantitative EEG results and information on cognitive functioning. Patients were included in the pooled analysis if sufficient data were reported in the article or provided by the author to allow analysis of individual treatment effect (i.e. if data on EEG or cognition were available before and after change in one treatment).

\section{Quality assessment / reduction of bias}

Case reports and small case series are likely to be influenced by publication and selection bias. To limit these influences, a subgroup analysis was performed including only studies reporting all consecutive patients fulfilling the inclusion criteria in a given specific period. This approach has been recommended by the Meta-analysis of Observational Studies in Epidemiology (MOOSE) study group.19 


\section{Data extraction and coding of data}

A standardized data-extraction form, containing 44 variables, was created. Patient characteristics before and after onset of ESES, treatment regimen under analysis (dosage, duration) and effects on cognitive function and EEG characteristics were included. Extraction of raw data from all studies was performed by one author (VvD). A random selection of $10 \%$ of relevant articles was checked by a second author (BvdM). There were some small differences in judgment that were settled by consensus meeting. None of these differences concerned the primary outcome (cognitive or EEG improvement).

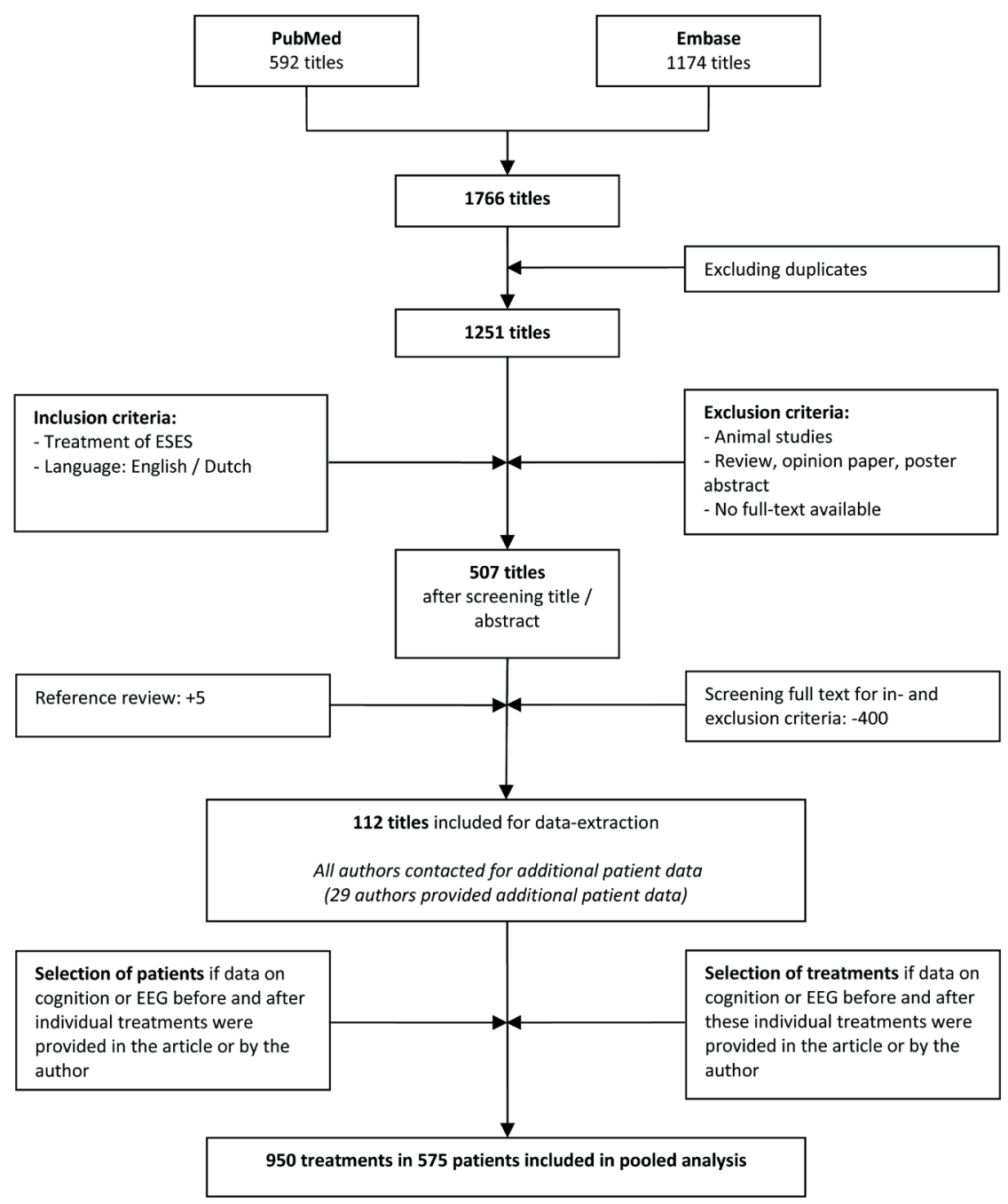

Figure 1: Flow chart search and selection process 


\section{Primary outcome assessment}

The three main outcome measures were improvement in cognitive function, EEG pattern and any improvement (cognition or EEG). To allow a pooled analysis all available data were coded according to the following criteria:

Cognition: improvement was defined primarily by the judgment of the author of the original article or as a gain of at least ten IQ points after start of treatment (when both pre- and post-treatment IQs were available).

EEG: improvement was primarily defined as an improvement of the sleep-induced status epilepticus pattern, as subjectively indicated by the author, or as a reduction of at least 25\% in Spike Wave Index (SWI, if pre- and post-treatment spike wave indices were given).

Any improvement: qualitative or quantitative improvement of either cognition, EEG or both.

Coding of data was performed by two authors: BvdM and FEJ for seizure type and EEG data. The other variables were coded by VvD and BvdM. In case of discordant judgment by the two reviewers the individual patient data were evaluated by a third independent reviewer (VvD or FEJ).

\section{Statistical analysis}

The baseline characteristics and effect of individual treatments were analyzed using IBM SPSS Statistics Software version 20. The baseline characteristics of the patients from consecutive cohorts were compared to those of the total study population, using the chi-square test for categorical variables (with Yates' Correction for Continuity for binomial variables) and the Mann-Whitney U-test for continuous variables (as these variables were not normally distributed). The same tests were applied to compare patients with known etiology to those with unknown etiology. The proportion of patients that showed improvement after the analyzed treatment is reported for all patients and for the subgroup of consecutive patients. The corresponding 95\% confidence intervals were calculated using normal approximation methods.

To identify variables that influence treatment outcome, a predictor analysis was performed with univariate and multivariate logistic regression analysis. Univariate analysis was performed for treatment category, gender, age at diagnosis, interval from diagnosis to treatment under evaluation, history of febrile seizures, mental development before the onset of ESES, presence of CT or MRI abnormalities and for the number of previous treatments. This analysis was first performed including only "complete cases" regarding 
included determinants, i.e. cases for which complete predictor data were available. To account for missing data a sensitivity analysis using multiple imputation methods was then performed; a procedure recommended for datasets with a significant proportion of missing data. ${ }^{20}$ All analyzed possible predictors with missing data were included in the model to allow an analysis of all treatments. Ten imputed datasets were created and a pooled analysis was carried out. The same procedure was used to perform a multivariate logistic regression analysis including treatment category and other possible predictors. There was a substantial proportion of missing data for most possible predictors. Therefore a multivariate analysis including only complete cases for all predictors would result in an analysis of only a small number of patients and is not reported.

\section{Results}

\section{Study and patient selection}

The search strategy and study and patient selection are shown in Figure 1. Descriptive information of the 112 included studies is available in table e-2. The included articles were published between 1977 and 2013. Some of these 112 articles showed overlap in patient population and were therefore included as 98 patient cohorts (grouping patients from the same center and integrating data of duplicate cases). From 94 of these 98 cohorts (96\%), cognitive outcome was reported, and from 88 (90\%) EEG outcome. The treatment categories that were analyzed were conventional anti-epileptic drugs in 48 cohorts, benzodiazepines in 23 cohorts, steroids in 41 cohorts, surgery in 18 cohorts and other treatments in 11 cohorts. The consecutive subgroup consisted of patients reported in 23 articles, which could be included as 15 different patient cohorts. The number of patients per included cohort was substantially higher in the consecutive subgroup (mean 23, median 10) compared to the total included population (mean 6, median 2).

Additional individual patient data were provided by 29 authors for 413 patients, of whom 675 individual treatments could be included in this analysis. By combining the data of 112 original articles with additional information provided by the authors, data of 575 patients could be included in whom 950 individual treatments and their effect were reported. The subgroup of consecutive patients consisted of 282 patients with 585 individual treatments.

\section{Patient characteristics}

A large majority (94\%) of patients had at least one seizure and their first seizure occurred at a median age of three and a half years, two and a half years prior to ESES diagnosis. Seizures were focal in $45 \%$, generalized in $21 \%$, and in $28 \%$ of patients both focal and generalized seizures were reported. Abnormal development was already present before 
Table 1. Patient characteristics

\begin{tabular}{|c|c|c|c|}
\hline & All patients $^{1}$ & $\begin{array}{l}\text { "Consecutive } \\
\text { patients"1,2 }\end{array}$ & $\begin{array}{l}p \text {-value for } \\
\text { comparison }^{3}\end{array}$ \\
\hline Number of patients & 575 & 282 & \\
\hline Male & $333 / 550(61 \%)$ & $166 / 282(59 \%)$ & 0.69 \\
\hline Abnormal perinatal history & $138 / 341(40 \%)$ & $118 / 256(46 \%)$ & 0.20 \\
\hline Positive family history for epilepsy & $49 / 238(21 \%)$ & $41 / 196(21 \%)$ & 1.00 \\
\hline History of Febrile seizures & $32 / 497(6 \%)$ & $22 / 204(11 \%)$ & 0.07 \\
\hline Mean / median age at seizure onset & $\begin{array}{c}44 / 42 \text { months } \\
(n=384)\end{array}$ & $\begin{array}{l}42 \text { / } 36 \text { months } \\
(n=212)\end{array}$ & 0.16 \\
\hline Mean / median age at developmental delay, arrest or regression & $\begin{array}{c}65 / 60 \text { months } \\
(n=306)\end{array}$ & $\begin{array}{c}68 / 63 \text { months } \\
(n=205)\end{array}$ & 0.24 \\
\hline Mean / median age at ESES diagnosis & $\begin{array}{c}75 / 72 \text { months } \\
(n=416)\end{array}$ & $\begin{array}{c}77 / 78 \text { months } \\
(n=240)\end{array}$ & 0.20 \\
\hline CT / MRI abnormalities & $213 / 461(46 \%)$ & $147 / 273(54 \%)$ & 0.05 \\
\hline Presumed Etiology: & & & 0.28 \\
\hline genetic & $29 / 432(7 \%)$ & $26 / 266(10 \%)$ & \\
\hline structural & $215 / 432(50 \%)$ & $136 / 266(51 \%)$ & \\
\hline metabolic & $2 / 432(1 \%)$ & $0 / 266(0 \%)$ & \\
\hline unknown & $186 / 432(43 \%)$ & $104 / 266(39 \%)$ & \\
\hline Abnormal development prior to ESES onset & $167 / 362(46 \%)$ & $141 / 261(54 \%)$ & 0.06 \\
\hline Type of (previous) seizures: & & & 0.00 \\
\hline none & $26 / 464(6 \%)$ & $7 / 261(3 \%)$ & \\
\hline focal & $208 / 464(45 \%)$ & $93 / 261(36 \%)$ & \\
\hline generalized & $98 / 464(21 \%)$ & $43 / 261(17 \%)$ & \\
\hline both & $132 / 464(28 \%)$ & $118 / 261(45 \%)$ & \\
\hline Frequency of seizures at inclusion: & & & 0.16 \\
\hline none & $43 / 261(17 \%)$ & $20 / 209(10 \%)$ & \\
\hline monthly & $97 / 261(37 \%)$ & $88 / 209(42 \%)$ & \\
\hline weekly & $37 / 261(14 \%)$ & $34 / 209(16 \%)$ & \\
\hline daily & $84 / 261(32 \%)$ & $67 / 209(32 \%)$ & \\
\hline Mean / median follow up duration after start of treatment & $\begin{array}{c}36 / 24 \text { months } \\
(n=349)\end{array}$ & $\begin{array}{c}36 / 24 \text { months } \\
(n=172)\end{array}$ & 0.15 \\
\hline Number of treatments & 950 & 585 & \\
\hline
\end{tabular}

\section{Legend:}

1. Patient characteristics are shown as number of patients with this characteristic / total number of patients for whom this characteristic is available and the corresponding percentage. 2. "Consecutive patients" = patients included from studies reporting all consecutive patients fulfilling the in- and exclusion criteria in a specified period. 3. P-values are reported for the comparison of baseline characteristics of the patients from consecutive cohorts to those of the total study population. The two groups were compared using the chi-square test for categorical variables (with Yates' Correction for Continuity for binomial variables) and the Mann-Whitney U-test for continuous variables.

ESES onset in $46 \%$ of patients. In $46 \%$ of all included patients, structural abnormalities were seen on CT or MRI, while a genetic or metabolic abnormality was reported in only a small proportion of patients ( $7 \%$ and $1 \%$ respectively). The available baseline characteristics of all patients, and of those reported consecutively, are displayed separately in Table 1. 
Except for a different distribution in seizure types, no significant differences between both groups were detected. A comparison between patients with known etiology and those with unknown etiology revealed a larger proportion with an abnormal perinatal history, abnormal development before ESES onset and CT / MRI abnormalities in patients with known etiology. These patients also had more frequent seizures and a smaller proportion of them had a positive family history for epilepsy (table e-3).

\section{Treatment response}

The overall treatment effect ("any improvement") is reported including 95\% Confidence Intervals in Table 2 . Any improvement was seen in 49\% of patients treated with conventional anti-epileptic drugs (AED) and in 68\% of patients treated with benzodiazepines. Treatment with steroids was associated with any improvement in $81 \%$ of patients, surgery in $90 \%$ and other treatments in 54\% of patients. Surgical techniques applied in 62 patients included multiple subpial transection $(n=31)$, hemispheric surgery $(n=13)$, corpus callosotomy $(n=9)$, lobar resection $(n=5)$, multilobar resection or disconnection $(n=2)$ and two other procedures (cysto-peritoneal shunting and one unspecified procedure). Cognitive improvement was seen after treatment with AED in 40\%, benzodiazepines in 50\%, steroids in $78 \%$ and after surgery in $80 \%$. EEG improvement was seen after treatment with AED in $45 \%$, benzodiazepines in 59\%, steroids in $70 \%$ and after surgery in $80 \%$ of the patients (Table 2).

Table 2: Reported treatment effect (percentage improved) in patients with encephalopathy with ESES

\begin{tabular}{|c|c|c|c|c|}
\hline & $\begin{array}{l}\text { All / Consecutive } \\
\text { subgroup }\end{array}$ & $\begin{array}{l}\text { Any improvement } \\
(95 \% \mathrm{CI})\end{array}$ & $\begin{array}{c}\text { Cognitive improvement* } \\
(95 \% \mathrm{Cl})\end{array}$ & $\begin{array}{c}\text { EEG improvement** } \\
(95 \% \mathrm{Cl})\end{array}$ \\
\hline \multirow{2}{*}{ AED } & All $(n=495)$ & $49 \%(44-53 \%)$ & $40 \%(23-31 \%)$ & $45 \%(41-50 \%)$ \\
\hline & Cons. $(n=310)$ & $34 \%(29-39 \%)$ & $32 \%(26-37 \%)$ & $33 \%(28-38 \%)$ \\
\hline \multirow{2}{*}{ Benzodiazepines } & All $(n=171)$ & $68 \%(61-75 \%)$ & $50 \%(42-59 \%)$ & $59 \%(52-67 \%)$ \\
\hline & Cons. $(n=107)$ & $59 \%(50-68 \%)$ & $45 \%(35-54 \%)$ & $46 \%(37-56 \%)$ \\
\hline \multirow{2}{*}{ Steroids } & All $(n=166)$ & $81 \%(75-87 \%)$ & $78 \%(72-85 \%)$ & $70 \%(62-77 \%)$ \\
\hline & Cons. $(n=100)$ & $75 \%(67-83 \%)$ & $70 \%(60-79 \%)$ & $68 \%(58-77 \%)$ \\
\hline \multirow{2}{*}{ Surgery } & All $(n=62)$ & $90 \%(83-98 \%)$ & $80 \%(70-90 \%)$ & $80 \%(68-91 \%)$ \\
\hline & Cons. $(n=30)$ & $93 \% * * *$ & $83 \%(70-97 \%)$ & $74 \%(58-91 \%)$ \\
\hline \multirow{2}{*}{ Other } & All $(n=56)$ & $54 \%(41-67 \%)$ & $67 \%(51-82 \%)$ & $29 \%(15-43 \%)$ \\
\hline & Cons. $(n=38)$ & $58 \%(42-74 \%)$ & $71 \%(53-89 \%)$ & $26 \%(8-44 \%)$ \\
\hline \multirow{2}{*}{ Total } & All $(n=950)$ & $61 \%(58-64 \%)$ & $53 \%(49-56 \%)$ & $54 \%(50-56 \%)$ \\
\hline & Cons. $(n=585)$ & $50 \%(46-54 \%)$ & $44 \%(40-49 \%)$ & $43 \%(39-47 \%)$ \\
\hline
\end{tabular}

Legend: $n=$ number of treatments analyzed, any improvement = improvement of cognition or EEG, missing $n=$ missing number of treatments for this outcome, $95 \% \mathrm{Cl}=95 \%$ confidence interval, $\mathrm{AED}=$ conventional anti-epileptic drugs, ${ }^{*}=$ for 126 (all patients) $/ 40$ (consecutive subgroup) treatments cognitive outcome was missing, ${ }^{* *}=$ for 68 (all patients) / 25 (consecutive subgroup) treatments EEG outcome was missing, ${ }^{* \star \star}=$ no $95 \%$ confidence interval mentioned due to small sample size and large proportion 
Of 771 included treatments (81\% of all included treatments) outcome data were separately reported for cognition and EEG (for the remainder only data on "any improvement" were available). In these cases any improvement was seen after 470 treatments (61\%). Of the 470 treatments that were associated with any improvement, 77 treatments (16\%) were associated with EEG improvement without cognitive improvement and 69 treatments (15\%) were associated with cognitive improvement without EEG improvement. After 324 treatments (69\%) both cognitive performance and EEG were reported to have improved. A subgroup analysis was performed on the 282 patients who were treated consecutively in reporting centers, with 585 individual treatments. In this subgroup improvement percentages were lower for AED (34\%), benzodiazepines (59\%) and steroids (75\%). The percentage of patients that improved after surgery was similar in this subgroup (93\%).

Table 3: Odds of treatment response (any improvement)

\begin{tabular}{|c|c|c|c|}
\hline Treatment & $\begin{array}{c}\text { OR }(95 \% \mathrm{Cl}) \\
\text { Univariate } \\
\text { (complete case) }\end{array}$ & $\begin{array}{l}\text { OR }(95 \% \mathrm{Cl}) \\
\text { Univariate } \\
\text { (MI) }\end{array}$ & $\begin{array}{l}\text { OR }(95 \% \text { CI }) \\
\text { Multivariate } \\
\text { (MI) }\end{array}$ \\
\hline AED & reference & reference & reference \\
\hline Benzodiazepines & $2.2(1.5-3.2)^{*}$ & $2.2(2.0-2.5)^{*}$ & $2.1(1.4-3.1)$ * \\
\hline Steroids & $4.4(2.9-6.7)^{*}$ & $4.4(3.9-5.0)^{*}$ & $4.2(2.7-6.5)$ * \\
\hline Surgery & $9.8(4.1-23.1)^{*}$ & $9.8(7.5-12.6)^{*}$ & $8.6(3.5-21.4)$ * \\
\hline Other & $1.2(0.69-2.1)$ & $1.2(1.0-1.4)^{*}$ & $1.1(0.6-2.0)$ \\
\hline \multicolumn{4}{|l|}{ Patient characteristics } \\
\hline Male gender & $1.2(0.9-1.6)$ & $1.2(0.9-1.5)$ & $1.4(1.0-1.8)^{*}$ \\
\hline Age at diagnosis & $1.0(1.0-1.0)$ & $1.0(1.0-1.0)$ & $1.0(1.0-1.0)$ \\
\hline Interval diagnosis - treatment & $1.0(1.0-1.0)$ & $1.0(1.0-1.0)$ & $1.0(1.0-1.0)$ \\
\hline Febrile seizures & $1.2(0.7-2.1)$ & $1.3(0.7-2.3)$ & $1.4(0.8-2.6)$ \\
\hline Abnormal development before ESES onset & $0.6(0.5-0.9)^{*}$ & $0.6(0.5-0.8)^{*}$ & $0.6(0.4-0.8)^{*}$ \\
\hline CT / MRI abnormalities & $0 . .8(0.6-1.1)$ & $0.8(0.6-1.1)$ & $1.0(0.7-1.4)$ \\
\hline CT / MRI abnormalities in non-surgically treated patients & $0.7(0.5-1.0)^{*}$ & $0.7(0.5-1.0)$ & $1.0(0.7-1.4)$ \\
\hline Number of previous treatments & $1.2(1.1-1.4)^{*}$ & $1.1(0.9-1.3)$ & $1.1(0.9-1.0)$ \\
\hline
\end{tabular}

Legend: $\mathrm{OR}=$ odds ratio, $\mathrm{AED}=$ conventional anti-epileptic drugs, complete case = complete case analysis, $95 \% \mathrm{Cl}=95 \%$ confidence interval, $\mathrm{Ml}=$ pooled analysis after using multiple imputation methods, ${ }^{*}=p<0.05$, Note: Multivariate analysis was performed using patient characteristics and treatment category as covariates

\section{Predictors of treatment response}

Subsequently, we analyzed possible predictors of treatment effect, including treatment category and patient characteristics (Table 3). "Any improvement" was associated with benzodiazepines, steroids and surgery when compared to treatment with AED. An abnormal development of patients prior to ESES onset was associated with no improvement after treatment. The presence of CT / MRI abnormalities in non-surgically treated patients and 
a lower number of previous treatments were significant predictors of poor treatment response in univariate analysis. However, this correlation did not sustain in multivariate analysis. These variables showed substantial differences among the treatment categories (table e-4). Male gender predicted better treatment response only in multivariate analysis of pooled data after multiple imputation. Separate analyses of possible predictors of EEG and cognitive outcome provided comparable results (table e-5).

\section{Discussion}

The results of this individual patient data meta-analysis suggest that treatment with steroids and surgery (in suitable candidates) are most effective in the treatment of ESES syndrome. Treatment success percentages (for EEG or cognitive improvement) were reported between 80 and 90\% for steroids and surgery and these results are relatively preserved in the consecutive subgroup analysis. Benzodiazepines may be considered an appropriate alternative with $69 \%$ and 59\% overall treatment success in all and consecutive patients respectively. Conventional anti-epileptic drugs were reported less effective in the included studies, with improvement seen in 49\% and 34\% of all patients and consecutive patients, respectively. Improvement most frequently reflected both EEG and cognitive outcomes. The strong influence of the treatment category chosen on treatment effect persists in a multivariate analysis with possible predictors. Among patient characteristics, developmental level before the onset of ESES was the only predictor with sustained significance in all analyses.

Normal development before the onset of ESES was found to be a significant predictor of treatment success and this association was significant in all analyses. This confirms earlier observations that $I Q$, presence and duration of cognitive impairment before the start of a treatment predict treatment response in patients with ESES. ${ }^{13,22,23}$ However, in many patients it is unclear if "pre-existent" abnormal development was unrelated to, or a first sign of "late recognized" ESES. Diagnostic delay is an important issue in children with epilepsy (especially in syndromes with non-convulsive seizures or status epilepticus) and is associated with more severe cognitive impairment. ${ }^{24}$ In our multivariate analysis the number of previous treatments was not associated with treatment efficacy. This contrasts to the results of a large study of anti-epileptic drugs for epilepsy in general, where a strong decrease in efficacy is seen after failure of a previous AED. ${ }^{25} \mathrm{~A}$ possible explanation is that our approach focuses on cognition and EEG abnormalities and not on seizure freedom. Some treatments may have been prescribed primarily to control seizures, although they were reported in the context of an ESES diagnosis. In general, we hypothesize that there is a tendency to initially prescribe a conventional anti-epileptic drug (especially in 
patients presenting with frequent seizures) or benzodiazepine and consider corticosteroids and surgery (often more successful) only in refractory cases. This hypothesis is supported by the observation that the mean number of previous treatment is higher for treatment with steroids and surgery than with AED in our dataset (table e-4). These differences could potentially result in higher success rates for the drugs prescribed later in the course of ESES syndrome. Overall, better outcomes have been reported in cases with unknown etiology, as compared to cases with structural lesions, except for surgically treated patients. ${ }^{11,13} \mathrm{We}$ found that a structural lesion predicts unsuccessful treatment in univariate analysis for non-surgical treatments. However, this association could not be confirmed in multivariate analysis.

The results have to be interpreted in the setting of data acquisition. Many of the included studies are small and retrospective. These case reports and small case series may have been published because of an exceptionally good or bad treatment effect, causing selection and publication bias. This is consistent with the observed differences in treatment effect among all patients and in the subgroup of consecutive patients. The only randomized controlled trial compared levetiracetam treatment with placebo and found a significant reduction in spikewave activity. No significant influence on cognitive performance was observed. The broad inclusion criteria of this trial (SWI > 30\%, many patients with only behavioral disturbances) and small sample size ( $n=18$ after exclusion of dropouts) limit the clinical relevance of these findings. This study could not be included in this meta-analysis as insufficient individual patient data were available. ${ }^{17,18}$ Additionally, the large heterogeneity of the included studies allowed analysis of qualitative outcome data only. Most authors clearly reported whether cognitive or EEG improvement was seen after treatment. We preferred to use quantitative data, however this was not possible due to missing quantitative data for a majority of patients. Furthermore, due to the limited size of the included studies it was not possible to account for heterogeneity between studies in the analysis. The analysis was limited to a fixed effect analysis of the individual patient data and data was treated as a single study dataset. Moreover, specific treatments that may differ in efficacy had to be clustered in our analysis, because the quality and quantity of data was insufficient for valid treatment-specific analysis. Furthermore, adverse effects and relapse rates are not included in the analysis because of missing data. Finally, we could not include treatment duration and dosage in the analysis as these were not reported in the majority of studies. Since follow-up periods are heterogeneous in different studies, we were not able to evaluate outcomes at comparable points in time. This point is particularly relevant in a disorder where natural fluctuations in severity occur over time. In this context, a positive or negative effect may be incorrectly attributed to a drug effect when it may have represented just a natural fluctuation in the course of the disease. Only prospective controlled trials with long follow-up will be able to distinguish natural fluctuations in the disease from treatment effects. 
The effect of treatment on seizure frequency was not included in this study, as we believe that cognitive disturbances and EEG abnormalities are the hallmarks of the syndrome and we considered these the most important outcomes of specific treatment for ESES syndrome.

Despite these challenges, this study provides valuable information. To our knowledge, it is the first pooled analysis of individual patient data evaluating the effect of different treatment regimens in patients with epileptic encephalopathy with ESES. The individual patient data meta-analysis approach allowed us to investigate the effect of a large number of individual treatments by combining the information from original articles with additional data provided by many authors. The adoption of an individual patient data analysis design has several advantages as compared to an aggregate analysis of literature reports only. Consistent in- and exclusion criteria can be used across studies and individual patients. In addition, individual patients' missing data can be observed and accounted for in the analysis at the individual level. Furthermore, studies that contain overlapping sets of patients can be identified and excluded from analyses, and the use of individual patient data enhances the possibilities of statistical testing. ${ }^{21}$ We performed a subgroup analysis of consecutive patients, reducing the effects of publication bias. Additionally, a predictor analysis was reported. By using multivariate logistic regression analysis we limited the influence of many possible confounders.

Our data suggest that surgery may be the most effective treatment for children with ESES syndrome. However, many of these surgically treated patients had a structural lesion and it is unlikely that surgery is equally effective in patients with ESES syndrome of unknown etiology, and it is unclear which technique can be applied in these patients. Multiple Subpial Transection (MST) has been suggested as a treatment option in Landau-Kleffner Syndrome, but evidence is limited and it is unclear whether MST can also be beneficial in ESES patients with cognitive deficits outside the language domain. ${ }^{26}$ Furthermore, adverse effects were not included in this analysis and reported qualitative improvement may not be sufficient to justify the risk of surgery. However, it is likely that surgery is underutilized and that surgery should be considered sooner in the treatment of ESES patients with regression of cognitive functioning and a structural lesion that is accessible for resection or disconnection.

In summary, this study provides new insights by combining the available evidence from mostly small and retrospective studies. Despite the large number of patients and treatments evaluated in the pooled analysis, no definite conclusions can be drawn on treatment effects. A very recent study showed that clinicians' approaches to the treatment of ESES syndrome differ a lot and although the current study is a step forward, no general recommendations can be made. ${ }^{27}$ Further research is urgently needed to provide definite 
answers regarding treatment of children with this, often devastating, epilepsy syndrome. A randomized controlled European multicenter trial was recently initiated and may provide further directions (RESCUE ESES, Randomized European trial of Steroids versus Clobazam Usage for Encephalopathy with Electrical Status Epilepticus in Sleep).

\section{Acknowledgements}

This work was supported by grants from the Dutch Epilepsy Fund and the Wilhelmina Children's Hospital Research Fund. The funding sources had no involvement in this study.

We thank Dr. Arts, Dr. Arvio, Dr. Aykut, Dr. Capovilla, Dr. Fejerman, prof. Guerrini, Dr. Haberlandt, Dr. Jocic-Jakubi, Dr. Kanemura, Dr. Kang, Dr. Kersbergen, Dr. Kelemen, Dr. Kossoff, Prof. Lagae, prof. Margari, Dr. Praline, Prof. Hommet, Dr. Raha, Dr. Tachikawa and Dr. You for contributing their individual patient data.

\section{Disclosures}

Dr. van den Munckhof, Dr. van Dee, Prof. Dr. Braun, and Dr. Jansen report grants from the Dutch Epilepsy Fund and Wilhelmina Children's Hospital Research Fund during the conduct of the study. Dr. Loddenkemper reports to receive research support from the American Epilepsy Society, the Epilepsy Foundation of America, the Center for Integration of Medicine and Innovative Technology, the Epilepsy Therapy Project, the Pediatric Epilepsy Research Foundation, Citizens United for Research in Epilepsy, Danny-Did Foundation, and the HHV-6 Foundation, and from investigator initiated research grants from Lundbeck and Eisai. Dr. Sagi, Dr. Caraballo, Prof. Veggiotti, Dr. Liukkonen, Dr. Sánchez Fernández, Dr. Buzatu, and Dr. Bulteau have no disclosures to report. We confirm that we have read the Journal's position on issues involved in ethical publication and affirm that this report is consistent with those guidelines. 


\section{Reference List}

1. Patry G, Lyagoubi S, Tassinari CA. Subclinical "electrical status epilepticus" induced by sleep in children. A clinical and electroencephalographic study of six cases. Arch Neurol 1971; 24:242-252.

2. Proposal for revised classification of epilepsies and epileptic syndromes. Commission on Classification and Terminology of the International League Against Epilepsy. Epilepsia 1989; 30:389399.

3. Hughes JR. A review of the relationships between Landau-Kleffner syndrome, electrical status epilepticus during sleep, and continuous spike-waves during sleep. Epilepsy Behav 2011; 20:247253.

4. Nickels K, Wirrell E. Electrical status epilepticus in sleep. Semin Pediatr Neurol 2008; 15:50-60.

5. Sanchez F, I, Loddenkemper T, Peters JM et al. Electrical status epilepticus in sleep: clinical presentation and pathophysiology. Pediatr Neurol 2012; 47:390-410.

6. Landau WM, Kleffner FR. Syndrome of acquired aphasia with convulsive disorder in children. Neurology 1957; 7:523-530.

7. Rossi PG, Parmeggiani A, Posar A et al. Landau-Kleffner syndrome (LKS): long-term follow-up and links with electrical status epilepticus during sleep (ESES). Brain Dev 1999; 21:90-98.

8. Lesca G, Rudolf G, Bruneau N et al. GRIN2A mutations in acquired epileptic aphasia and related childhood focal epilepsies and encephalopathies with speech and language dysfunction. Nat Genet 2013; 45:1061-1066.

9. Lemke JR, Lal D, Reinthaler EM et al. Mutations in GRIN2A cause idiopathic focal epilepsy with rolandic spikes. Nat Genet 2013; 45:1067-1072.

10. Carvill GL, Regan BM, Yendle SC et al. GRIN2A mutations cause epilepsy-aphasia spectrum disorders. Nat Genet 2013; 45:1073-1076.

11. Caraballo RH, Veggiotti P, Kaltenmeier MC et al. Encephalopathy with status epilepticus during sleep or continuous spikes and waves during slow sleep syndrome: a multicenter, long-term follow-up study of 117 patients. Epilepsy Res 2013; 105:164-173.

12. Saltik S, Uluduz D, Cokar O et al. A clinical and EEG study on idiopathic partial epilepsies with evolution into ESES spectrum disorders. Epilepsia 2005; 46:524-533.

13. Liukkonen E, Kantola-Sorsa E, Paetau R et al. Long-term outcome of 32 children with encephalopathy with status epilepticus during sleep, or ESES syndrome. Epilepsia 2010; 51:2023-2032.

14. Perniola $T$, Margari $L$, Buttiglione $M$ et al. A case of Landau-Kleffner syndrome secondary to inflammatory demyelinating disease. Epilepsia 1993; 34:551-556.

15. Scholtes FB, Hendriks MP, Renier WO. Cognitive deterioration and electrical status epilepticus during slow sleep. Epilepsy Behav 2005; 6:167-173.

16. Chin RF, Neville BG, Peckham C et al. Treatment of community-onset, childhood convulsive status epilepticus: a prospective, population-based study. Lancet Neurol 2008; 7:696-703.

17. Larsson PG, Bakke KA, Bjornaes $\mathrm{H}$ et al. The effect of levetiracetam on focal nocturnal epileptiform activity during sleep--a placebo-controlled double-blind cross-over study. Epilepsy Behav 2012; 24:44-48.

18. Bjornaes H, Bakke KA, Larsson PG et al. Subclinical epileptiform activity in children with electrical status epilepticus during sleep: effects on cognition and behavior before and after treatment with levetiracetam. Epilepsy Behav 2013; 27:40-48.

19. Stroup DF, Berlin JA, Morton SC et al. Meta-analysis of observational studies in epidemiology: a proposal for reporting. Meta-analysis Of Observational Studies in Epidemiology (MOOSE) group. JAMA 2000; 283:2008-2012.

20. Donders AR, van der Heijden GJ, Stijnen T et al. Review: a gentle introduction to imputation of missing values. J Clin Epidemiol 2006; 59:1087-1091. 
Chapter 6. Treatment of ESES

21. Riley RD, Lambert PC, Abo-Zaid G. Meta-analysis of individual participant data: rationale, conduct, and reporting. BMJ 2010; 340:c221.

22. Buzatu M, Bulteau C, Altuzarra C et al. Corticosteroids as treatment of epileptic syndromes with continuous spike-waves during slow-wave sleep. Epilepsia 2009; 50 Suppl 7:68-72.

23. Kramer U, Sagi L, Goldberg-Stern $\mathrm{H}$ et al. Clinical spectrum and medical treatment of children with electrical status epilepticus in sleep (ESES). Epilepsia 2009; 50:1517-1524.

24. Berg AT, Loddenkemper T, Baca CB. Diagnostic delays in children with early onset epilepsy: impact, reasons, and opportunities to improve care. Epilepsia 2014; 55:123-132.

25. Schiller Y, Najjar Y. Quantifying the response to antiepileptic drugs: effect of past treatment history. Neurology 2008; 70:54-65.

26. Cross JH, Neville BG. The surgical treatment of Landau-Kleffner syndrome. Epilepsia 2009; 50 Suppl 7:63-67.

27. Sanchez F, I, Chapman K, Peters JM et al. Treatment for continuous spikes and waves during sleep (CSWS): Survey on treatment choices in North America. Epilepsia 2014;55(7):1099-1108. 


\section{Supplementary data}

Table e-1: Search Strategy in PubMed and Embase (subsequent rows were linked by "OR")

\begin{tabular}{|c|c|}
\hline $\begin{array}{l}\text { (Electric OR Electrics OR Electrical) AND (Status OR static OR } \\
\text { statics) AND sleep }\end{array}$ & $\begin{array}{l}\text { Treatment OR Treatments OR Therapy OR Therapies OR } \\
\text { Therapeutic }\end{array}$ \\
\hline $\begin{array}{l}\text { Continuous AND (spike OR spikes) AND (wave OR waves) AND } \\
\text { sleep }\end{array}$ & Anticonvulsant $\mathrm{OR}$ anticonvulsants \\
\hline \multirow{8}{*}{$\begin{array}{l}\text { Landau Kleffner OR (acquired epileptic aphasia) OR (acquired } \\
\text { epileptiform aphasia) OR (acquired aphasia AND convulsive } \\
\text { disorder) OR (acquired aphasia with convulsive disorder) OR } \\
\text { (acquired auditory verbal agnosia) }\end{array}$} & Antiepileptic OR antiepileptics OR anti-epileptic OR anti-epileptics \\
\hline & benzodiazepine $\mathrm{OR}$ benzodiazepines \\
\hline & Steroid OR Steroids OR Corticosteroid OR Corticosteroids \\
\hline & Prednison OR prednisone OR prednisolon OR prednisolone \\
\hline & $\begin{array}{l}\text { Methylprednison OR methylprednisone OR methylprednisolon OR } \\
\text { Methylprednisolone }\end{array}$ \\
\hline & ACTH \\
\hline & Immunoglobulins OR IVIG \\
\hline & Ketogenic \\
\hline
\end{tabular}


Table e-2: Included studies

\begin{tabular}{|c|c|c|c|c|c|c|c|c|}
\hline Author & $\begin{array}{l}\text { Year } \\
\text { (of } \\
\text { publication) }\end{array}$ & Study design & $\begin{array}{l}\text { Consecutive } \\
\text { patients } \\
\text { (Yes / No) }\end{array}$ & $\begin{array}{l}\text { Patients } \\
\text { with ESES/ } \\
\text { LKS (n) }\end{array}$ & $\begin{array}{l}\text { Treatments } \\
\text { analyzed } \\
\text { (n) }\end{array}$ & $\begin{array}{l}\text { Treatment } \\
\text { category } \\
\text { included }\end{array}$ & $\begin{array}{l}\text { Outcome } \\
\text { measure }\end{array}$ & $\begin{array}{l}\text { Additional } \\
\text { information } \\
\text { provided } \\
\text { (Yes / No) }\end{array}$ \\
\hline Aeby & 2005 & retrospective & N & 12 & 12 & AED & EEG, cognition & $\mathrm{N}$ \\
\hline Ansink & 1988 & retrospective & N & 1 & 1 & AED & cognition & N \\
\hline Appleton & 1993 & retrospective & N & 1 & 1 & AED & $\begin{array}{l}\text { EEG, cognition, } \\
\text { seizures }\end{array}$ & N \\
\hline Arts & 2009 & prospective & Y & 6 & 14 & $\begin{array}{l}\text { IVIG, steroids, } \\
\text { ketogenic } \\
\text { diet }\end{array}$ & $\begin{array}{l}\text { EEG, cognition, } \\
\text { seizures }\end{array}$ & Y \\
\hline Arvio & 2005 & prospective & N & 3 & 3 & AED & $\begin{array}{l}\text { cognition, } \\
\text { seizures }\end{array}$ & Y \\
\hline Atkins & 2011 & prospective & N & 20 & 20 & AED & EEG & N \\
\hline Aykut & 1996 & retrospective & N & 1 & 1 & steroids & $\begin{array}{l}\text { cognition, } \\
\text { seizures }\end{array}$ & Y \\
\hline Battaglia & 2003 & retrospective & N & 1 & 1 & AED & EEG, cognition & N \\
\hline Battaglia & 2009 & retrospective & N & 2 & 2 & surgery & $\begin{array}{l}\text { EEG, cognition, } \\
\text { seizures }\end{array}$ & N \\
\hline Bergqvist & 1999 & retrospective & N & 3 & 3 & ketogenic diet & $\begin{array}{l}\text { EEG, cognition, } \\
\text { seizures }\end{array}$ & N \\
\hline Boel & 1989 & retrospective & N & 1 & 6 & AED, BZD & $\begin{array}{l}\text { EEG, cognition, } \\
\text { seizures }\end{array}$ & N \\
\hline Buisson & 2006 & retrospective & N & 2 & 3 & $\begin{array}{l}\text { steroids, } \\
\text { surgery }\end{array}$ & $\begin{array}{l}\text { EEG, cognition, } \\
\text { seizures }\end{array}$ & N \\
\hline Buzatu & 2009 & retrospective & Y & 44 & 44 & steroids & $\begin{array}{l}\text { EEG, cognition, } \\
\text { seizures }\end{array}$ & Y \\
\hline Capovilla & 2004 & retrospective & N & 3 & 3 & AED & $\begin{array}{l}\text { EEG, cognition, } \\
\text { seizures }\end{array}$ & Y \\
\hline $\begin{array}{l}\text { Caraballo } \\
\text { (5 articles) }\end{array}$ & $\begin{array}{l}2001 / 2008 \text { / } \\
2010 / 2011 / \\
2013\end{array}$ & retrospective & Y & 117 & 169 & $\begin{array}{l}\text { AED, BZD, } \\
\text { steroids }\end{array}$ & $\begin{array}{l}\text { EEG, cognition, } \\
\text { seizures }\end{array}$ & Y \\
\hline Castillo & 2008 & retrospective & N & 1 & 2 & $\begin{array}{l}\text { ketogenic } \\
\text { diet, surgery }\end{array}$ & $\begin{array}{l}\text { EEG, cognition, } \\
\text { seizures }\end{array}$ & $N$ \\
\hline Chhun & 2011 & prospective & N & 6 & 6 & AED & $\begin{array}{l}\text { EEG, cognition, } \\
\text { seizures }\end{array}$ & $N$ \\
\hline $\begin{array}{l}\text { Coutinho dos } \\
\text { Santos }\end{array}$ & 2002 & retrospective & N & 2 & 2 & AED, steroids & $\begin{array}{l}\text { EEG, cognition, } \\
\text { seizures }\end{array}$ & $\mathrm{N}$ \\
\hline De Negri & 1995 & prospective & N & 16 & 16 & BZD & EEG, cognition & N \\
\hline De Volder & 1994 & retrospective & $\mathrm{N}$ & 1 & 2 & $\begin{array}{l}\text { steroids, } \\
\text { surgery }\end{array}$ & $\begin{array}{l}\text { EEG, cognition, } \\
\text { seizures }\end{array}$ & $N$ \\
\hline Deuel & 1977 & retrospective & $\mathrm{N}$ & 1 & 4 & AED, BZD & $\begin{array}{l}\text { EEG, cognition, } \\
\text { seizures }\end{array}$ & N \\
\hline Fandino & 2011 & retrospective & N & 3 & 4 & AED, steroids & $\begin{array}{l}\text { EEG, cognition, } \\
\text { seizures }\end{array}$ & N \\
\hline Fejerman & 2012 & retrospective & N & 53 & 53 & AED & $\begin{array}{l}\text { EEG, cognition, } \\
\text { seizures }\end{array}$ & Y \\
\hline Gallagher & 2006 & retrospective & $\mathrm{N}$ & 1 & 2 & steroids & $\begin{array}{l}\text { EEG, cognition, } \\
\text { seizures }\end{array}$ & $\mathrm{N}$ \\
\hline
\end{tabular}




\begin{tabular}{|c|c|c|c|c|c|c|c|c|}
\hline Author & $\begin{array}{l}\text { Year } \\
\text { (of } \\
\text { publication) }\end{array}$ & Study design & $\begin{array}{l}\text { Consecutive } \\
\text { patients } \\
\text { (Yes / No) }\end{array}$ & $\begin{array}{l}\text { Patients } \\
\text { with ESES/ } \\
\text { LKS }(n)\end{array}$ & $\begin{array}{l}\text { Treatments } \\
\text { analyzed } \\
\text { (n) }\end{array}$ & $\begin{array}{l}\text { Treatment } \\
\text { category } \\
\text { included }\end{array}$ & $\begin{array}{l}\text { Outcome } \\
\text { measure }\end{array}$ & $\begin{array}{l}\text { Additional } \\
\text { information } \\
\text { provided } \\
\text { (Yes / No) }\end{array}$ \\
\hline Grote & 1999 & retrospective & $N$ & 14 & 14 & surgery & EEG, cognition & $\mathrm{N}$ \\
\hline Guerrini & 1998 & retrospective & N & 3 & 3 & AED & $\begin{array}{l}\text { EEG, cognition, } \\
\text { seizures }\end{array}$ & Y \\
\hline Haberlandt & 2010 & retrospective & $\mathrm{N}$ & 5 & 5 & steroids & $\begin{array}{l}\text { EEG, cognition, } \\
\text { seizures }\end{array}$ & Y \\
\hline $\begin{array}{l}\text { Hirsch/ } \\
\text { Marescaux }\end{array}$ & 1990 & retrospective & N & 5 & 28 & $\begin{array}{l}\text { AED, BZD, } \\
\text { steroids }\end{array}$ & $\begin{array}{l}\text { EEG, cognition, } \\
\text { seizures }\end{array}$ & N \\
\hline Hoppen & 2003 & retrospective & N & 1 & 1 & AED & $\begin{array}{l}\text { EEG, cognition, } \\
\text { seizures }\end{array}$ & N \\
\hline Huppke & 2005 & retrospective & N & 1 & 1 & AED & $\begin{array}{l}\text { EEG, cognition, } \\
\text { seizures }\end{array}$ & N \\
\hline Inutsuka & 2006 & retrospective & N & 13 & 20 & $\begin{array}{l}\text { AED, BZD, } \\
\text { steroids }\end{array}$ & EEG & N \\
\hline Irwin & 2001 & retrospective & Y & 5 & 7 & $\begin{array}{l}\text { steroids, } \\
\text { surgery }\end{array}$ & $\begin{array}{l}\text { EEG, cognition, } \\
\text { seizures }\end{array}$ & N \\
\hline Jocic-Jakubi & 2007 & retrospective & N & 1 & 1 & BZD & $\begin{array}{l}\text { EEG, cognition, } \\
\text { seizures }\end{array}$ & Y \\
\hline Kanemura & 2009 & retrospective & $\mathrm{N}$ & 1 & 1 & BZD & $\begin{array}{l}\text { EEG, cognition, } \\
\text { seizures }\end{array}$ & Y \\
\hline Kanemura & 2013 & prospective & Y & 1 & 1 & AED & $\begin{array}{l}\text { EEG, cognition, } \\
\text { seizures }\end{array}$ & Y \\
\hline Kang & 2006 & retrospective & N & 1 & 1 & ketogenic diet & $\begin{array}{l}\text { EEG, cognition, } \\
\text { seizures }\end{array}$ & Y \\
\hline Kersbergen & 2013 & prospective & Y & 4 & 13 & $\begin{array}{l}\text { AED, BZD, } \\
\text { steroids, IVIG }\end{array}$ & $\begin{array}{l}\text { EEG, cognition, } \\
\text { seizures }\end{array}$ & Y \\
\hline Kelemen & 2006 & retrospective & N & 1 & 1 & steroids & $\begin{array}{l}\text { EEG, cognition, } \\
\text { seizures }\end{array}$ & Y \\
\hline Kellerman & 1978 & retrospective & N & 1 & 1 & steroids & cognition & N \\
\hline Kossoff & 2003 & retrospective & N & 1 & 1 & AED & EEG, cognition & Y \\
\hline $\begin{array}{l}\text { Kramer / } \\
\text { Kramer / Geva- } \\
\text { Dayan }\end{array}$ & $\begin{array}{l}2006 / 2009 / \\
2012\end{array}$ & retrospective & Y & 30 & 212 & $\begin{array}{l}\text { AED, BZD, } \\
\text { steroids, IVIG } \\
\text { surgery }\end{array}$ & EEG, cognition & Y \\
\hline Lagae & 1998 & retrospective & N & 1 & 3 & steroids, IVIG & $\begin{array}{l}\text { EEG, cognition, } \\
\text { seizures }\end{array}$ & Y \\
\hline Lanzi & 1994 & retrospective & N & 1 & 3 & steroids & $\begin{array}{l}\text { EEG, cognition, } \\
\text { seizures }\end{array}$ & N \\
\hline Lerman & 1991 & retrospective & N & 4 & 7 & AED, steroids & $\begin{array}{l}\text { EEG, cognition, } \\
\text { seizures }\end{array}$ & N \\
\hline Li & 1996 & retrospective & N & 3 & 5 & $\begin{array}{l}\text { AED, BZD, } \\
\text { steroids }\end{array}$ & $\begin{array}{l}\text { EEG, cognition, } \\
\text { seizures }\end{array}$ & N \\
\hline Liukkonen & 2010 & prospective & Y & 32 & 32 & $\begin{array}{l}\text { AED, BZD, } \\
\text { steroids }\end{array}$ & $\begin{array}{l}\text { EEG, cognition, } \\
\text { seizures }\end{array}$ & Y \\
\hline Loddenkemper & 2009 & retrospective & Y & 8 & 8 & surgery & $\begin{array}{l}\text { EEG, cognition, } \\
\text { seizures }\end{array}$ & Y \\
\hline Maquet & 1995 & retrospective & N & 2 & 4 & BZD, steroids & $\begin{array}{l}\text { EEG, cognition, } \\
\text { seizures }\end{array}$ & N \\
\hline
\end{tabular}




\begin{tabular}{|c|c|c|c|c|c|c|c|c|}
\hline Author & $\begin{array}{l}\text { Year } \\
\text { (of } \\
\text { publication) }\end{array}$ & Study design & $\begin{array}{l}\text { Consecutive } \\
\text { patients } \\
\text { (Yes / No) }\end{array}$ & $\begin{array}{l}\text { Patients } \\
\text { with ESES/ } \\
\text { LKS (n) }\end{array}$ & $\begin{array}{l}\text { Treatments } \\
\text { analyzed } \\
\text { (n) }\end{array}$ & $\begin{array}{l}\text { Treatment } \\
\text { category } \\
\text { included }\end{array}$ & $\begin{array}{l}\text { Outcome } \\
\text { measure }\end{array}$ & $\begin{array}{l}\text { Additional } \\
\text { information } \\
\text { provided } \\
\text { (Yes / No) }\end{array}$ \\
\hline Margari & 2012 & prospective & $Y$ & 25 & 17 & AED & $\begin{array}{l}\text { EEG, cognition, } \\
\text { seizures }\end{array}$ & Y \\
\hline $\begin{array}{l}\text { Mikati/Fayad/ } \\
\text { Mikati }\end{array}$ & $\begin{array}{l}2002 / 1997 / \\
2000\end{array}$ & prospective & Y & 5 & 5 & IVIG & $\begin{array}{l}\text { EEG, cognition, } \\
\text { seizures }\end{array}$ & N \\
\hline Monteiro & 2001 & retrospective & N & 1 & 1 & steroids & $\begin{array}{l}\text { EEG, cognition, } \\
\text { seizures }\end{array}$ & N \\
\hline Morrell & 1995 & prospective & $\mathrm{N}$ & 3 & 3 & surgery & $\begin{array}{l}\text { cognition, } \\
\text { seizures }\end{array}$ & N \\
\hline Mosely & 2012 & retrospective & $\mathrm{N}$ & 1 & 1 & surgery & $\begin{array}{l}\text { EEG, cognition, } \\
\text { seizures }\end{array}$ & N \\
\hline Nakayama & 2012 & retrospective & N & 1 & 1 & AED & EEG, seizures & N \\
\hline Nass & 1993 & retrospective & N & 1 & 1 & surgery & $\begin{array}{l}\text { EEG, cognition, } \\
\text { seizures }\end{array}$ & N \\
\hline Neville & 1997 & retrospective & N & 1 & 2 & $\begin{array}{l}\text { steroids, } \\
\text { surgery }\end{array}$ & $\begin{array}{l}\text { cognition, } \\
\text { seizures }\end{array}$ & N \\
\hline Nikanorova & 2009 & prospective & $\mathrm{N}$ & 5 & 5 & ketogenic diet & EEG, cognition & N \\
\hline Okayuz & 2005 & retrospective & $\mathrm{N}$ & 1 & 1 & steroids & $\begin{array}{l}\text { EEG, cognition, } \\
\text { seizures }\end{array}$ & N \\
\hline Paquier & 1992 & prospective & $\mathrm{N}$ & 3 & 3 & AED, steroids & $\begin{array}{l}\text { cognition, } \\
\text { seizures }\end{array}$ & N \\
\hline Paquier & 2009 & retrospective & N & 2 & 3 & AED, BZD & $\begin{array}{l}\text { EEG, cognition, } \\
\text { seizures }\end{array}$ & N \\
\hline $\begin{array}{l}\text { Pascual- } \\
\text { Castroviejo }\end{array}$ & 1992 & retrospective & N & 3 & 4 & AED, steroids & $\begin{array}{l}\text { EEG, cognition, } \\
\text { seizures }\end{array}$ & N \\
\hline Peltola & 2011 & retrospective & Y & 13 & 13 & surgery & $\begin{array}{l}\text { EEG, cognition, } \\
\text { seizures }\end{array}$ & Y \\
\hline Perniola & 1993 & retrospective & N & 1 & 1 & steroids & $\begin{array}{l}\text { EEG, cognition, } \\
\text { seizures }\end{array}$ & N \\
\hline $\begin{array}{l}\text { Praline/ } \\
\text { Hommet }\end{array}$ & $2003 / 2000$ & retrospective & $\mathrm{N}$ & 7 & 14 & AED, BZD & $\begin{array}{l}\text { EEG, cognition, } \\
\text { seizures }\end{array}$ & Y \\
\hline Praline & 2006 & retrospective & N & 1 & 1 & BZD & EEG, cognition & Y \\
\hline Prats & 1998 & retrospective & $\mathrm{N}$ & 5 & 5 & AED, BZD & $\begin{array}{l}\text { EEG, cognition, } \\
\text { seizures }\end{array}$ & N \\
\hline Raha & 2012 & prospective & Y & 10 & 18 & $\begin{array}{l}\text { steroids, IVIG, } \\
\text { ketogenic } \\
\text { diet }\end{array}$ & EEG, cognition & Y \\
\hline Rinthaka & 1995 & retrospective & N & 1 & 2 & AED, steroids & $\begin{array}{l}\text { EEG, cognition, } \\
\text { seizures }\end{array}$ & N \\
\hline Roulet-Perez & 1991 & retrospective & N & 1 & 1 & AED & $\begin{array}{l}\text { EEG, cognition, } \\
\text { seizures }\end{array}$ & N \\
\hline Roulet-Perez & 1993 & prospective & N & 1 & 1 & AED & EEG, cognition & N \\
\hline Roulet-Perez & 1998 & retrospective & N & 1 & 2 & AED, surgery & $\begin{array}{l}\text { EEG, cognition, } \\
\text { seizures }\end{array}$ & N \\
\hline $\begin{array}{l}\text { Roulet-Perez I } \\
\text { Kallay }\end{array}$ & 2010/2009 & prospective & $\mathrm{N}$ & 1 & 1 & surgery & $\begin{array}{l}\text { EEG, cognition, } \\
\text { seizures }\end{array}$ & N \\
\hline
\end{tabular}




\begin{tabular}{|c|c|c|c|c|c|c|c|c|}
\hline Author & $\begin{array}{l}\begin{array}{l}\text { Year } \\
\text { (of } \\
\text { publication) }\end{array}\end{array}$ & Study design & $\begin{array}{l}\text { Consecutive } \\
\text { patients } \\
\text { (Yes / No) }\end{array}$ & $\begin{array}{l}\text { Patients } \\
\text { with ESES/ } \\
\text { LKS (n) }\end{array}$ & $\begin{array}{l}\text { Treatments } \\
\text { analyzed } \\
\text { (n) }\end{array}$ & $\begin{array}{l}\text { Treatment } \\
\text { category } \\
\text { included }\end{array}$ & $\begin{array}{l}\text { Outcome } \\
\text { measure }\end{array}$ & $\begin{array}{l}\text { Additional } \\
\text { information } \\
\text { provided } \\
\text { (Yes / No) }\end{array}$ \\
\hline Saaldeldin & 2011 & retrospective & N & 1 & 1 & AED & $\begin{array}{l}\text { EEG, cognition, } \\
\text { seizures }\end{array}$ & $N$ \\
\hline $\begin{array}{l}\text { Sanchez } \\
\text { Fernandez - } \\
\text { Epilepsia }\end{array}$ & 2012 & retrospective & Y & 9 & 9 & $\begin{array}{l}\text { AED, BZD, } \\
\text { steroids, IVIG, } \\
\text { VNS }\end{array}$ & $\begin{array}{l}\text { EEG, cognition, } \\
\text { seizures }\end{array}$ & Y \\
\hline $\begin{array}{l}\text { Sanchez } \\
\text { Fernandez } \\
\text {-Pediatric } \\
\text { Neurology }\end{array}$ & 2012 & retrospective & Y & 29 & 29 & BZD & $\begin{array}{l}\text { EEG, cognition, } \\
\text { seizures }\end{array}$ & Y \\
\hline Sawhney 1995 & 1995 & retrospective & N & 3 & 3 & surgery & $\begin{array}{l}\text { EEG, cognition, } \\
\text { seizures }\end{array}$ & N \\
\hline Sayit & 1999 & retrospective & N & 1 & 1 & steroids & $\begin{array}{l}\text { EEG, cognition, } \\
\text { seizures }\end{array}$ & N \\
\hline Seegmuller & 2012 & retrospective & N & 1 & 1 & BZD & cognition & N \\
\hline Sinclair & 2005 & prospective & N & 10 & 10 & steroids & $\begin{array}{l}\text { EEG, cognition, } \\
\text { seizures }\end{array}$ & N \\
\hline Stroink & 1997 & retrospective & N & 1 & 1 & steroids & $\begin{array}{l}\text { EEG, cognition, } \\
\text { seizures }\end{array}$ & N \\
\hline Stulpnagel & 2010 & retrospective & N & 6 & 6 & AED & $\begin{array}{l}\text { EEG, cognition, } \\
\text { seizures }\end{array}$ & N \\
\hline Tachikawa & 2001 & retrospective & N & 1 & 3 & AED, steroids & $\begin{array}{l}\text { EEG, cognition, } \\
\text { seizures }\end{array}$ & Y \\
\hline Tharpe & 1991 & retrospective & N & 1 & 1 & AED & cognition & N \\
\hline Tohyama & 2011 & retrospective & N & 1 & 1 & steroids & cognition & N \\
\hline Tsuru & 2000 & retrospective & $\mathrm{N}$ & 2 & 5 & $\begin{array}{l}\text { AED, BZD, } \\
\text { steroids }\end{array}$ & $\begin{array}{l}\text { EEG, cognition, } \\
\text { seizures }\end{array}$ & N \\
\hline Uldall & 2000 & retrospective & N & 1 & 2 & steroids & $\begin{array}{l}\text { EEG, cognition, } \\
\text { seizures }\end{array}$ & N \\
\hline $\begin{array}{l}\text { van de Sandt- } \\
\text { Koenderman }\end{array}$ & 1984 & retrospective & N & 1 & 1 & AED & EEG, cognition & N \\
\hline $\begin{array}{l}\text { van Hirtum- } \\
\text { Das }\end{array}$ & 2006 & retrospective & N & 1 & 1 & surgery & $\begin{array}{l}\text { EEG, cognition, } \\
\text { seizures }\end{array}$ & N \\
\hline Varga & 2011 & prospective & N & 5 & 5 & $\begin{array}{l}\text { transcranial } \\
\text { direct current } \\
\text { stimulation }\end{array}$ & EEG & N \\
\hline Vigliano & 2010 & retrospective & N & 1 & 1 & surgery & $\begin{array}{l}\text { cognition, } \\
\text { seizures }\end{array}$ & N \\
\hline Wakai & 1997 & retrospective & N & 1 & 1 & AED & $\begin{array}{l}\text { EEG, cognition, } \\
\text { seizures }\end{array}$ & N \\
\hline Wang & 2008 & prospective & N & 5 & 5 & AED & $\begin{array}{l}\text { EEG, cognition, } \\
\text { seizures }\end{array}$ & N \\
\hline Wirrel & 2006 & retrospective & N & 1 & 1 & AED & $\begin{array}{l}\text { EEG, cognition, } \\
\text { seizures }\end{array}$ & N \\
\hline Yan Liu & 2000 & retrospective & N & 9 & 9 & BZD & $\begin{array}{l}\text { EEG, cognition, } \\
\text { seizures }\end{array}$ & N \\
\hline
\end{tabular}




\begin{tabular}{|c|c|c|c|c|c|c|c|c|}
\hline Author & $\begin{array}{l}\text { Year } \\
\text { (of } \\
\text { publication) }\end{array}$ & Study design & $\begin{array}{l}\text { Consecutive } \\
\text { patients } \\
\text { (Yes/ No) }\end{array}$ & $\begin{array}{l}\text { Patients } \\
\text { with ESES/ } \\
\text { LKS (n) }\end{array}$ & $\begin{array}{l}\text { Treatments } \\
\text { analyzed } \\
\text { (n) }\end{array}$ & $\begin{array}{l}\text { Treatment } \\
\text { category } \\
\text { included }\end{array}$ & $\begin{array}{l}\text { Outcome } \\
\text { measure }\end{array}$ & $\begin{array}{l}\text { Additional } \\
\text { information } \\
\text { provided } \\
\text { (Yes / No) }\end{array}$ \\
\hline Yasuhara & 1991 & retrospective & $N$ & 2 & 2 & BZD & $\begin{array}{l}\text { EEG, cognition, } \\
\text { seizures }\end{array}$ & $N$ \\
\hline You & 2008 & retrospective & N & 2 & 2 & steroids & $\begin{array}{l}\text { EEG, cognition, } \\
\text { seizures }\end{array}$ & Y \\
\hline Zardini & 1994 & retrospective & N & 1 & 1 & steroids & $\begin{array}{l}\text { EEG, cognition, } \\
\text { seizures }\end{array}$ & N \\
\hline Zhang & 2010 & retrospective & N & 1 & 1 & AED & $\begin{array}{l}\text { EEG, cognition, } \\
\text { seizures }\end{array}$ & N \\
\hline
\end{tabular}

Legend: Author: If more than one author is mentioned, the included patients were described in more than one article. Study design: reported as prospective if this is clear from the article or additional information by the author. Otherwise reported as retrospective. Treatment category included: AED = conventional anti-epileptic drugs, BZD = benzodiazepines

Table e-3: Distribution of patient characteristics among patients with known versus unknown etiology

\begin{tabular}{|c|c|c|c|}
\hline & $\begin{array}{c}\text { Patients with } \\
\text { known etiology* }\end{array}$ & $\begin{array}{c}\text { Patients with } \\
\text { unknown etiology* }\end{array}$ & $\begin{array}{l}\mathrm{p} \text {-value for } \\
\text { comparison }\end{array}$ \\
\hline Number of patients & 246 & 186 & \\
\hline Male & $144 / 244(59 \%)$ & $113 / 186(61 \%)$ & 0.79 \\
\hline Abnormal perinatal history & $115 / 183(63 \%)$ & $14 / 95(15 \%)$ & 0.00 \\
\hline Positive family history for epilepsy & $20 / 135(15 \%)$ & $22 / 74(30 \%)$ & 0.02 \\
\hline History of Febrile seizures & $14 / 205(7 \%)$ & $10 / 149(7 \%)$ & 1.00 \\
\hline Mean / median age at seizure onset & $\begin{array}{c}35 / 33 \text { months } \\
(n=199)\end{array}$ & $\begin{array}{l}52 / 48 \text { months } \\
(n=111)\end{array}$ & 0.00 \\
\hline Mean / median age at developmental delay, arrest or regression & $\begin{array}{l}67 / 61 \text { months } \\
(n=148)\end{array}$ & $\begin{array}{c}70 / 70 \text { months } \\
(n=77)\end{array}$ & 0.31 \\
\hline Mean / median age at ESES diagnosis & $\begin{array}{c}75 / 72 \text { months } \\
(n=210)\end{array}$ & $\begin{array}{c}76 / 78 \text { months } \\
(n=136)\end{array}$ & 0.81 \\
\hline CT / MRI abnormalities & $206 / 233(88 \%)$ & $6 / 150(4 \%)$ & 0.00 \\
\hline Abnormal development prior to ESES onset & $118 / 177(67 \%)$ & $41 / 108(38 \%)$ & 0.00 \\
\hline $\begin{array}{l}\text { Type of (previous) seizures: } \\
\text { none } \\
\text { focal } \\
\text { generalized } \\
\text { both }\end{array}$ & $\begin{array}{c}2 / 228(1 \%) \\
109 / 228(48 \%) \\
38 / 228(17 \%) \\
79 / 228(35 \%)\end{array}$ & $\begin{array}{c}4 / 145(3 \%) \\
69 / 145(48 \%) \\
23 / 145(16 \%) \\
49 / 145(34 \%)\end{array}$ & 0.57 \\
\hline $\begin{array}{l}\text { Frequency of seizures at inclusion: } \\
\text { none } \\
\text { monthly } \\
\text { weekly } \\
\text { daily }\end{array}$ & $\begin{array}{l}6 / 143(4 \%) \\
60 / 143(42 \%) \\
22 / 143(15 \%) \\
55 / 143(39 \%)\end{array}$ & $\begin{array}{l}19 / 87(24 \%) \\
30 / 81(37 \%) \\
13 / 81(16 \%) \\
19 / 81(24 \%)\end{array}$ & 0.00 \\
\hline Mean / median follow up duration after start of treatment & $\begin{array}{l}26 / 24 \\
(n=15)\end{array}$ & $\begin{array}{c}29 / 25 \text { weeks } \\
(n=17)\end{array}$ & 0.33 \\
\hline
\end{tabular}

Legend: * In 246 patients with known etiology a genetic etiology was reported in 29, a structural etiology in 215 and a metabolic etiology in 2 patients. In 186 patient an unknown etiology was reported and for 143 patient no data regarding etiology was available. 
Table e-4: Distribution of significant predictors in univariate analysis, not in multivariate analysis among treatment categories

\begin{tabular}{lcc}
\hline Treatment & $\begin{array}{c}\text { CT / MRI Abnormalities (\% after } \\
\text { multiple imputation) }\end{array}$ & $\begin{array}{c}\text { Mean number of previous treatments } \\
\text { (after multiple imputation) }\end{array}$ \\
\hline AED $(n=495)$ & $45 \%$ & 2.7 \\
Benzodiazepines $(n=171)$ & $42 \%$ & 3.0 \\
Steroids $(n=166)$ & $34 \%$ & 3.0 \\
Surgery $(n=62)$ & $58 \%$ & 4.0 \\
Other $(n=56)$ & $33 \%$ & 3.1 \\
\hline
\end{tabular}

Legend: $A E D$ = conventional anti-epileptic drugs, note: Multivariate analysis was performed using the patient characteristics and treatment category as covariates.

Table e-5: Predictors of treatment response (cognition, EEG

\begin{tabular}{lcc}
\hline Patient characteristics & $\begin{array}{c}\text { Cognition } \\
\text { OR (95\% CI) Multivariate (MI) }\end{array}$ & $\begin{array}{c}\text { EEG } \\
\text { OR (95\% CI) Multivariate (MI) }\end{array}$ \\
\hline Male gender & $1.3(1.0-1.7)$ & $\mathbf{1 . 4}(\mathbf{1 . 1}-\mathbf{1 . 9})$ \\
Age at diagnosis & $1.0(1.0-1.0)$ & $1.0(1.0-1.0)$ \\
Interval diagnosis - treatment & $1.0(1.0-1.0)$ & $1.0(1.0-1.0)$ \\
Febrile seizures & $1.4(0.8-2.4)$ & $1.6(0.8-3.1)$ \\
Abnormal development before ESES onset & $\mathbf{0 . 6 ( 0 . 4 - 0 . 8 )}$ & $\mathbf{0 . 5}(\mathbf{0 . 4 - 0 . 7 )}$ \\
CT / MRI abnormalities & $0.7(0.5-1.0)$ & $1.0(0.7-1.5)$ \\
Number of previous treatments & $1.1(1.0-1.3)$ & $1.1(0.9-1.3)$
\end{tabular}

Legend: $\mathrm{OR}=$ odds ratio, $95 \% \mathrm{Cl}=95 \%$ confidence interval, $\mathrm{MI}=$ pooled analysis after using multiple imputation methods, note: Multivariate analysis was performed using the patient characteristics and treatment category as covariates. 

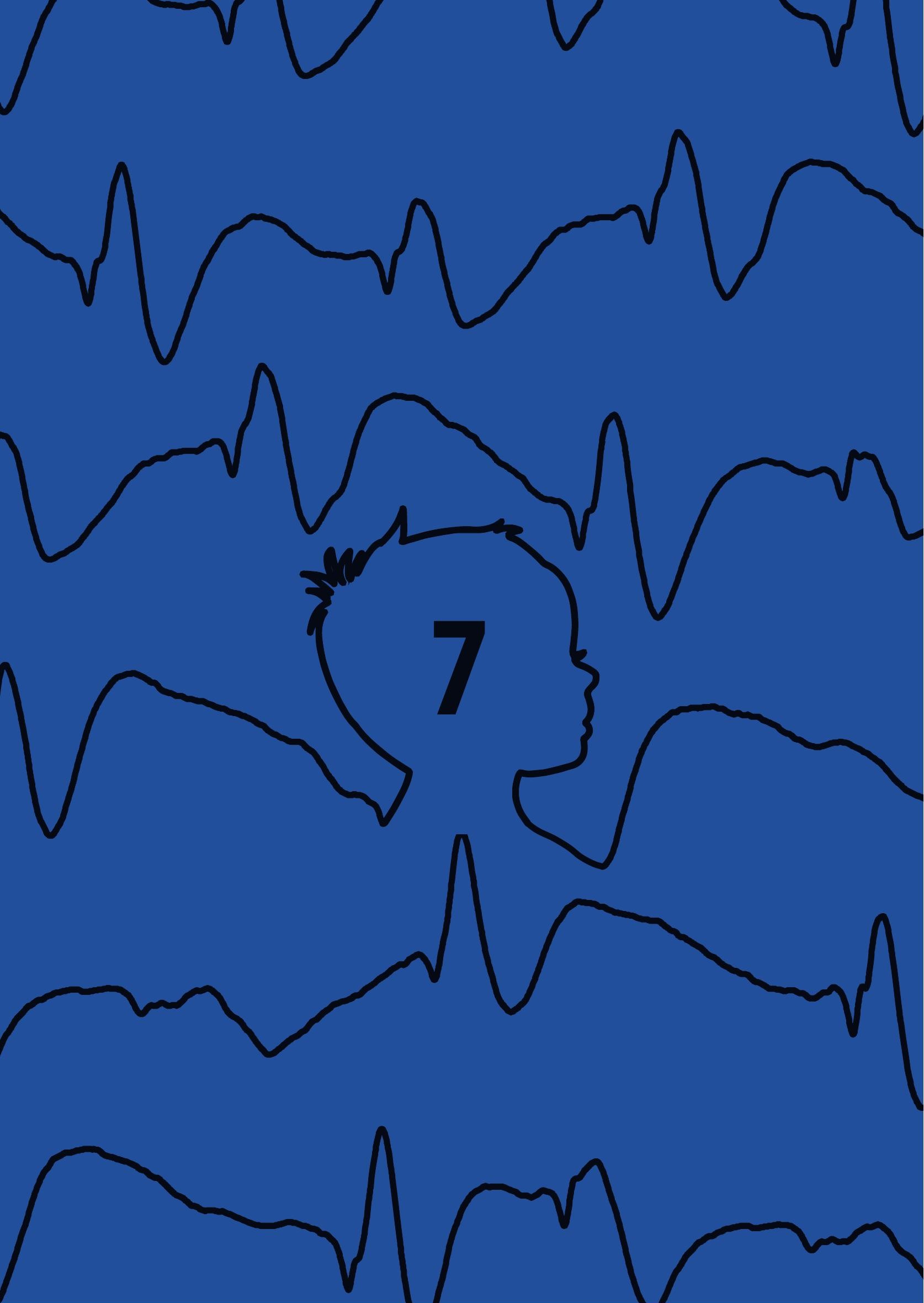


\title{
Chapter 7
}

\section{Treatment of electrical status epilepticus in sleep: clinical and EEG characteristics and response to 147 treatments in 47 patients}

\author{
Bart van den Munckhof \\ Christian Alderweireld \\ Susanne Davelaar \\ Heleen C. van Teeseling \\ Stavros Nikolakopoulos \\ Kees P.J. Braun \\ Floor E. Jansen
}




\section{Abstract}

Objective: Electrical status epilepticus in sleep (ESES) syndrome is characterized by near-continuous sleep-induced epileptiform activity and acquired cognitive deficits. Treatment is assumed mandatory to improve cognitive outcome. We aimed to compare EEG characteristics, subjective evaluation and objective neuropsychological assessment as measures to evaluate treatment efficacy, and to analyze possible predictors.

Methods: We retrospectively included patients with ESES syndrome treated in our center. Treatment effect was analyzed on sleep EEG spike wave index (SWI) and cognitive functioning.

Results: 47 patients had 147 (43 steroid and 104 non-steroid) treatments. Cognitive improvement was reported after 36\% of treatments at first follow-up and $45 \%$ of treatments at last follow-up. The median SWI change for treatments resulting in subjective cognitive improvement was $-44 \%$, and $0 \%$ for those not resulting in subjective cognitive improvement at first follow-up ( $p=0.008)$ and $-50 \%$ vs. $+5 \%$ at last follow-up ( $p=0.002)$. No clear association between subjective cognitive improvement and IQ change, and between SWI and IQ change was found. By means of logistic regression we found that steroid treatment, as compared to non-steroid treatment, was associated with cognitive improvement at first follow-up (multivariate OR after multiple imputation 2.5, 95\% Cl 1.15.7), while at last follow-up, higher age at diagnosis was related to cognitive improvement only in univariate analysis (OR 1.02, 95\% Cl 1.01-1.04).

Conclusions: We found that in children with ESES, cognitive improvement after treatment was strongly associated with SWI decrease, while it was not reflected by a significant IQ increase. Steroid treatment was most successful in improving cognitive performance.

\section{Highlights}

- Treatment of ESES syndrome aims to improve cognitive outcome.

- Improvement of daily functioning after treatment was strongly associated with SWI decrease.

- Improvement of daily functioning and SWI change were not reflected by IQ change.

- Steroid treatment was most successful in improving cognitive performance.

- Higher age at diagnosis was related to cognitive improvement after treatment. 


\section{Introduction}

Encephalopathy with electrical status epilepticus in sleep (ESES), ESES syndrome and continuous spikes and waves during sleep (CSWS) have been used interchangeably to define patients with an EEG pattern of near-continuous spike and wave discharges during non-REM sleep and acquired neuropsychological deficits. Typical cases have a spike wave index (SWI) of at least 85\% during non-REM sleep, while in recent years cases with a SWI of $50-85 \%$ were added to the spectrum.[1-5] This epilepsy syndrome is typically age related, presenting at an age between 2 and 14 years, with a peak at 4 to 8 years. Although seizures are present in the majority of patients and can form a serious burden, cognitive decline is the most frightening symptom of the disorder.[2,6,7] While the ESES EEG pattern resolves during puberty, cognitive deficits often remain.[2]

Structural brain abnormalities have been reported in 20-50\% of patients with ESES and an important role of the thalamus has become evident from recent studies.[8-12] Also, etiological as well as treatment studies have linked inflammation to ESES.[13,14] How ESES leads to cognitive decline is incompletely understood. The epileptiform activity has been suggested to interfere with normal recuperative functions of sleep, thereby adversely affecting learning abilities, language, memory and other cognitive domains.[15,16]

Early and adequate treatment of ESES is assumed mandatory to prevent further cognitive decline and possibly recover skills that were lost. A recent study has shown that treatment strategies vary widely between clinicians. [17] No adequately powered randomized controlled trials are available and evidence is limited to mostly small and retrospective case series. A pooled analysis of 575 cases reported in literature revealed that conventional anti-epileptic drugs are often not successful in improving cognitive outcome, while benzodiazepines and steroids seem better alternatives. Surgery is highly successful in selected cases. [8] Although a causative relation is assumed, it is unclear whether resolution of the ESES EEG pattern is necessary for treatment efficacy or can serve as a predictive biomarker.[18] Cognitive improvement should be leading, but its assessment is often based on subjective measures (parents' opinion, clinician's judgment).

In a large, single center cohort study of patients with ESES syndrome we aimed to address the following questions: (1) What is the effect of treatment on cognitive functioning and EEG-abnormalities? (2) Is there an association between EEG response to treatment, measured as a change of the spike-wave index, subjective cognitive functioning and IQ test results?, and (3) what are predictors of cognitive outcome? 


\section{Methods}

\section{Patients}

We retrospectively selected all children with epileptic encephalopathy with ESES, further called ESES syndrome, who consulted the pediatric neurology clinic of the UMC Utrecht, the Netherlands between January 2002 and December 2013. The study was approved by the medical ethics committee who judged that the Dutch Medical Research Involving Human Subjects Act did not apply.

Patients were selected according to predefined inclusion criteria: 1) a diagnosis of ESES syndrome before the age of 12 years, since improvement of the ESES EEG pattern afterward is expected in the natural course of the disorder,[19] 2) availability of a diagnostic EEG performed during sleep (either a whole night EEG or a nap EEG after sleep deprivation) with a SWI during sleep of at least 50\% and 3) presence of acquired cognitive deficits or behavior disorders, including cases with a clear deterioration in the context of a pre-existing delay.

To analyze the effect of treatment on cognitive functioning and follow up EEG, the treatments were included if they fulfilled the following criteria: 1) follow-up duration of at least one month after start of treatment (without another concurrent treatment change), 2) availability of a sleep deprived or whole night EEG before and after this treatment and 3) availability of information on (subjectively or objectively assessed) cognitive functioning and behavior before and after the treatment. Treatments were excluded from the analysis if they were given before ESES was diagnosed, if more than one pharmacologic treatment was started at the same time or if the treatment was given at a subtherapeutic dose.

\section{Clinical data collection and coding}

Baseline data on gender, perinatal history, etiology (MRI, genetic and metabolic test results), neurodevelopment, behavior, history of febrile seizures, family history of epilepsy, the date of diagnosis of ESES, the age at diagnosis of ESES, presence of seizures, age at seizure onset, seizure semiology, the IQ at diagnosis of ESES and the total number of treatments for ESES was extracted from the medical charts.

In addition the following data was collected for individual treatments: date at start and stop of treatment, duration of treatment, treatment type and category (AED, benzodiazepines, steroids, surgery, IVIG or other), dosages, and number of previous treatments for ESES. If a patient was treated with steroids, the first follow-up after completing the intended schedule (in most cases 6 monthly methylprednisolone pulses) was considered first follow-up after treatment. If a patient was treated with daily oral steroids, any follow-up visit at least one month after treatment initiation was considered the first follow up. If 
multiple different steroid regimens were given in one patient, each was counted as a single treatment (e.g. 6 methylprednisolone pulses and a year later oral prednisolone treatment were included as two separate steroid treatments). For each treatment, information on cognitive functioning (based on neuropsychological assessment, when available, or based on the report of the treating doctor), behavioral problems (scored as mild, moderate or severe according to Massa et al)[20] and seizure frequency before and after treatment were collected. The follow-up results after a treatment were collected for both first-follow up after reaching the intended dosage as well as last follow-up before the start of another treatment or the last follow-up before May 1, 2014. If only one follow-up visit was available, this was included as the first follow-up after treatment.

\section{EEG data acquisition and quantification of epileptiform activity}

All EEGs were recorded at 21 scalp electrodes according to the international 10-20 system. All EEG data was collected using the SystemPlus Evolution Micromed software package. SWIs of EEGs were calculated by an experienced EEG technician (SD) or other researchers (CA or BvdM) and 20\% was checked by an experienced epileptologist (FEJ).

The SWI of each EEG was calculated in an epoch of ten minutes (600 seconds) duration, starting five minutes after the alpha attenuation or after sleep clinically had commenced. The number of seconds containing epileptiform discharges was divided by the total number of seconds in the epoch (600) and multiplied by 100 to reflect the SWI as a percentage.

\section{Outcome definition}

Primary outcomes for this study were change in cognitive functioning and SWI change. Subjective improvement of cognitive functioning was defined as present or absent based on medical chart review (parents' and clinician's judgment). If a neuropsychological assessment was available before and after an individual treatment, total IQ data were also used for analysis of treatment efficacy. Secondary outcomes were change in behavior, seizure frequency and side-effects of treatment.

\section{Statistical analysis}

The SWI and IQ data were assessed for normality with Q-Q plots and with a ShapiroWilk test. Based on the distribution of the data, either a parametric (student's t-test) or non-parametric test (Mann-Whitney U-test) was used for comparison of two groups. The agreement between the SWIs calculated by two researchers was assessed with a BlandAltman plot and a two dependent samples test. Two independent samples tests were used to investigate the possible association between change in subjectively assessed cognitive functioning, IQ change and SWI change. 
A complete case logistic regression analysis was performed to investigate possible predictors of cognitive improvement after treatment. Baseline variables that were considered of potential relevance were included in a univariate analysis. We also performed multiple imputation in SPSS (regression method) to create 10 imputed datasets using these variables and the output variables to account for missing data. Based on the results of the univariate analysis after multiple imputation, the variables with a p-value below 0.2 were subsequently entered in a multivariate model.

\section{Results}

\section{Patient characteristics}

Of 57 patients who were diagnosed with ESES syndrome, $47(82,5 \%)$ met the inclusion criteria. Nine patients were excluded because SWIs did not exceed 50\%, one patient never received treatment. The mean age at diagnosis of the included patients was 6,8 years and a male preponderance was seen $(61,7 \%)$. Twenty-two patients had MRI abnormalities: 13 patients had vascular abnormalities, 7 patients had developmental malformations and 2 patients had hippocampal sclerosis. Genetic abnormalities included a KCNB1 mutation and copy number variants on chromosome 5, 9, 715 and 16. In the 47 included patients 148 treatments were given. One treatment was excluded because insufficient clinical data was available. The patient and treatment selection process is shown in figure 1. Baseline characteristics of the included patients are shown in table 1.

Table 1: Baseline patient characteristics $(n=47)$

\begin{tabular}{lc}
\hline mean / median age at ESES diagnosis (years, SD) & $\mathbf{6 . 8}$ / $\mathbf{6 . 8}$ (2.3), $\mathbf{n = 4 7}$ \\
\hline male gender & $29(62 \%)$ \\
complicated pregnancy & $11 / 47(23 \%)$ \\
complicated delivery & $17 / 47(36 \%)$ \\
abnormal MRI & $22 / 45(49 \%)$ \\
abnormal genetic testing & $9 / 28(32 \%)$ \\
history of febrile seizure & $9 / 45(20 \%)$ \\
family history positive for epilepsy / seizures & $8 / 47(17 \%)$ \\
abnormal development before ESES diagnosis & $18 / 45(40 \%)$ \\
mean / median total IQ at diagnosis (SD) & $76 / 76(22), \mathrm{n}=44$ \\
abnormal motor development & $14 / 47(30 \%)$ \\
abnormal language development & $16 / 47(34 \%)$ \\
behavioral deficit* & $36 / 46(78 \%)$ \\
any seizures & $42 / 45(93 \%)$ \\
\hline
\end{tabular}

Legend:

For categorical variables the number of patients with this finding and the total number of patients for whom this variable is available are shown. Percentages shown represent the proportion of the patients for which this variable is available. * Behavioral deficit as reported at time of ESES diagnosis. 


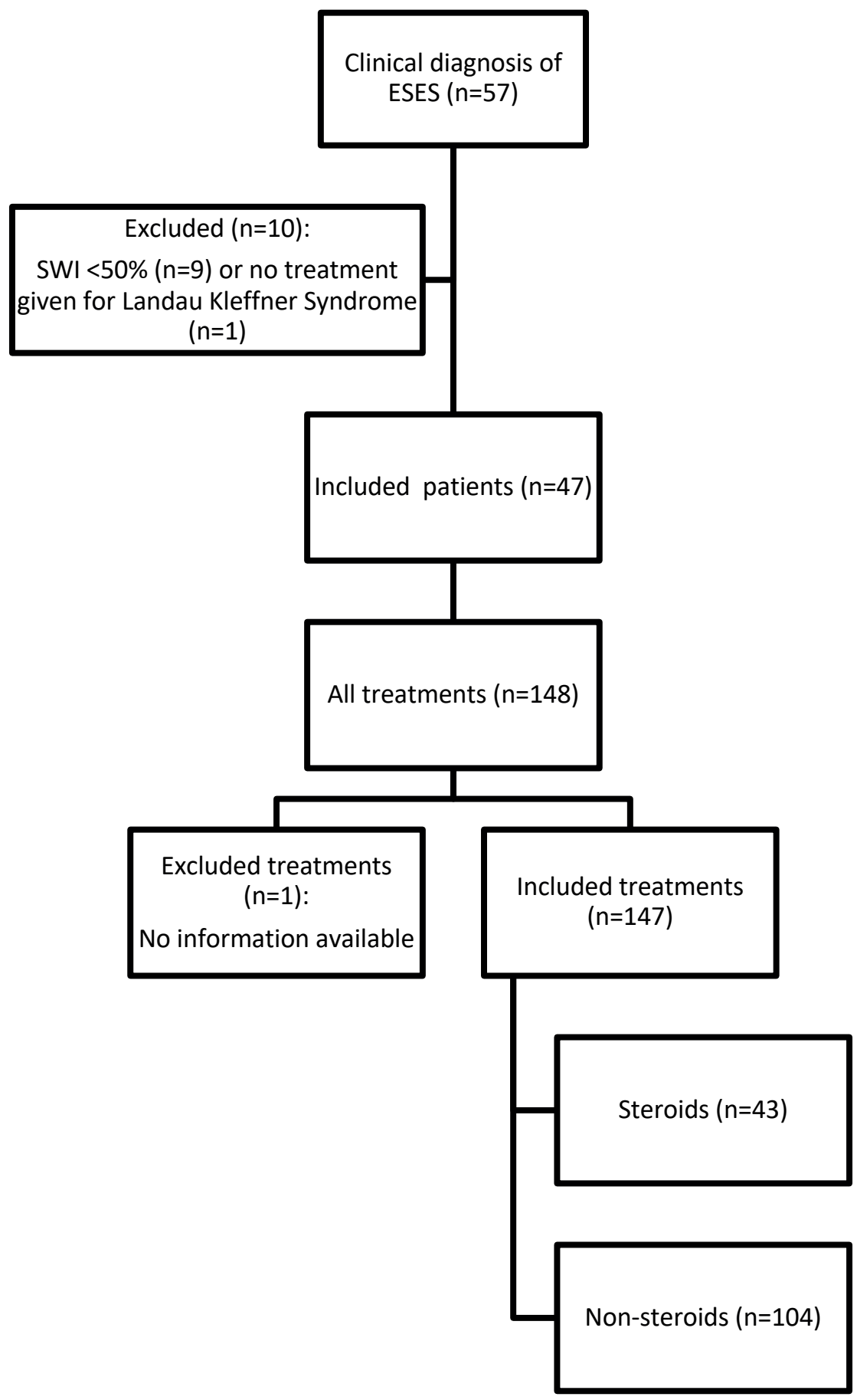

Figure 1: Flow chart patient and treatment selection 


\section{Effect of treatment on cognitive performance and EEG abnormalities}

The effect of treatment on cognitive performance was assessable for 119 (81\%) treatments at first follow-up (mean follow-up 4.7 months) and 94 (63,9\%) at last follow-up (mean follow-up 13.1 months). Cognitive improvement was reported after $36 \%$ of treatments at first follow-up and $45 \%$ of treatments at last follow-up. For those treatments for which an IQ before and after this individual treatment was available, the mean change in IQ was -1.7 points (median $-0.5, \mathrm{n}=40$ ) at first follow-up and -1.5 points (median $-2.0, \mathrm{n}=11$ ) at last follow-up.

The calculation of spike-wave indices showed good agreement between researchers ( $p$ for Wilcoxon signed ranks test 0,655 ). The median SWI change (compared to before treatment) was -5\% (mean-21\%) at first follow-up and -24\% (mean-31\%) at last follow-up.

A subgroup analysis was performed, including only the first treatments after the diagnosis of ESES $(n=44)$. At first follow-up after first treatments, subjective cognitive improvement was reported in $46 \%$ (17 of 37), and the median change in spike-wave index was -23\% (mean -32\%, n=30). At last follow-up after first treatments cognitive improvement was reported in 57\% (16 of 28) and the median change in spike-wave index was - $21 \%$ (mean $-26 \%, n=15$ ). For those patients for whom an IQ test was available both before and after this first treatment, not interfered by another treatment change, the median IQ change was 0 (mean -1, n=21). If any IQ measurement after treatment (regardless of other treatments) is considered, the median IQ change was -1 (mean $-1, \mathrm{n}=28$ ).

\section{Association of subjectively reported cognitive improvement and measured IQ change}

For all treatments $(n=33)$ where IQ scores were available both before and after therapy, we correlated the subjective evaluation by parents with the objectively measured change in IQ scores.(table 2) At first follow up, treatments that - according to parents - led to improvement were associated with a median IQ change of only +2.0 points (mean - 0.4 ). Those without subjective improvement were associated with a median IQ change of -7 (mean -4.5, p=0,32). At last follow-up, IQ scores and subjective data both before and after therapy were available for only 8 treatments (after 7 of these treatments cognitive improvement was reported) and no significant association was found.

\section{Association of subjectively reported cognitive improvement and SWI change}

For all treatments where sleep EEGs were available both before and after therapy, we correlated the evaluation by parents with the change in SWI. (table 2) At first follow up $(n=62)$, treatments that - according to parents - led to improvement were associated with a median SWI change of $-44 \%$ (mean -40\%). Despite this decrease in SWI, 48\% had EEGs 
that still fulfilled the criteria of ESES (SWI > 50\%). Those without subjective improvement were associated with a median SWI change of 0\% (mean -8\%). The SWI change was significantly different in the patients with subjective cognitive improvement, compared to those without subjective improvement $(p=0.008)$. At last follow-up, treatments $(n=28)$ that - according to parents - led to cognitive improvement were associated with a median SWI change of $-50 \%$ (mean $-45 \%$ ), although 53\% still fulfilled the criteria of ESES. Those without subjective cognitive improvement were associated with a median SWI change of $+5 \%$ (mean $+4 \%)$. Again, this difference is clearly significant $(p=0.002)$.

Table 2: Comparison of IQ change and SWI change between patients who showed subjective cognitive improvement at first follow-up and patients who did not

\begin{tabular}{|c|c|c|c|c|c|}
\hline & & \multicolumn{2}{|c|}{ first follow-up } & \multicolumn{2}{|c|}{ last follow-up } \\
\hline & & $\begin{array}{c}\text { IQ change } \\
\text { (mean / median) }\end{array}$ & $\begin{array}{c}\text { SWI change } \\
\text { (mean / median) }\end{array}$ & $\begin{array}{c}\text { IQ change } \\
\text { (mean / median) }\end{array}$ & $\begin{array}{c}\text { SWI change } \\
\text { (mean / median) }\end{array}$ \\
\hline \multirow{4}{*}{ Cognitive improvement } & Yes & $-0.4 /+2.0$ & $-40 \% /-44 \%$ & $-4.2 /-2.0$ & $-45 \% /-50 \%$ \\
\hline & $n$ & $18 / 43$ & $25 / 43$ & $7 / 42$ & $21 / 42$ \\
\hline & No & $-4.5 /-7.0$ & $-8 \% / 0.0 \%$ & $+2.0 /+2.0$ & $+4 \% /+5 \%$ \\
\hline & $n$ & $15 / 76$ & $37 / 76$ & $1 / 52$ & $7 / 52$ \\
\hline $\mathbf{P}_{\text {between groups }}$ & & 0.32 & 0.008 & 0.42 & 0.002 \\
\hline \multicolumn{6}{|c|}{$\begin{array}{l}\text { n: number of treatments for which both subjective cognitive data and objective data (IQ / SWI) are available / total number of } \\
\text { treatments. EEG data were more widely available than IQ data, resulting in higher numbers that could be analyzed. } \\
\text { *: due to the limited availability of IQ data at last follow-up the comparison was based on only } 8 \text { patients ( } 7 \text { with subjectively reported } \\
\text { improvement, } 1 \text { without subjectively reported improvement) }\end{array}$} \\
\hline
\end{tabular}

\section{Correlation between IQ (change) and SWI (change)}

In a comparison of baseline IQ and baseline SWI (before the first treatment was started) no significant correlation was found (Spearman's Rho 0.155, $p=0.397$ ). The treatments for which IQ and SWI were available both before and after treatment, were analyzed for possible correlations between SWI change and IQ change after treatment. No significant correlation was found at first follow-up (Spearman's Rho 0.251, $p=0.166, n=32$ ). At last follow-up a significant positive correlation was found between SWI change and IQ change (Spearman's Rho 0.857, $p=0.014, n=7$ ).

\section{Reported effect of different treatment categories}

The first treatment prescribed after the diagnosis of ESES was a conventional anti-epileptic drug (AED) in 10 patients, a benzodiazepine in 21 children, a corticosteroid in 14 and another treatment in one child. Subjective cognitive improvement at first follow-up was reported in $43 \%$ of the children first treated with AED, in $37 \%$ of those first treated with 
a benzodiazepine, and $64 \%$ of patients first treated with a corticosteroid. At last followup (prior to subsequent treatments) this was 50\%, 50\% and $83 \%$ of patients respectively (supplementary table 1). IQ before and after the first treatment, without another interfering treatment, was available for only a limited number of patients. Therefore, we also included the difference in IQ (delta IQ) before and after the first treatment regardless of other treatments (i.e. the first IQ after the first treatment and the last available IQ compared to the baseline IQ before the first treatment) in supplementary table 1. EEG showed a median spike-wave index change of $+3 \%$ (AED), $-9 \%$ (benzodiazepines) and -56\% (corticosteroids) at first follow-up and $+6 \%$ (AED), $-4 \%$ (benzodiazepines) and $-58 \%$ (corticosteroids) at last follow-up. (supplementary table 2)

If all subsequent treatments are also included in the analysis, 35 AED treatments (most often levetiracetam) were associated with subjective cognitive improvement in $23 \%$ at first follow up (46\% at last follow-up), 39 benzodiazepine treatments (most often clobazam) with 32\% at first follow-up (36\% at last follow-up), and 43 corticosteroid (methylprednisolone, prednisolone or dexamethasone) treatments with cognitive improvement in 53\% at first follow-up (58\% at last follow-up). Surgery was associated with reported cognitive improvement in 1 of 2 cases (50\%) at first follow-up (100\% at last follow-up) and intravenous immunoglobulins in 4 of 12 treatments (33\%) at first followup (36\% at last follow-up). A detailed overview of cognitive performance and SWI change after all treatments $(n=147)$ at first and at last follow-up can be found in supplementary table 3 .

\section{Predictors of subjectively assessed cognitive improvement after treatment}

With univariate analysis we found that at first follow-up after a specific treatment, the number of previous treatments (OR 0,78; $95 \% \mathrm{Cl} 0.64-0.95)$ and the use of corticosteroids compared to AED (OR 3.7; 95\% Cl 1.2 - 11.4)) were significantly associated with cognitive improvement and this finding was confirmed in the univariate analysis after multiple imputation. (table 3) In a subsequent multivariate analysis after multiple imputation, only the use of corticosteroids (compared to any other treatment category) was significantly associated with cognitive improvement at first follow-up (OR 2.5; 95\% Cl 1.1 - 5.7). The influence of the number of previous treatments on cognitive improvement at first follow-up of treatment is displayed in figure 2. A decreasing trend in proportion of subjective cognitive improvement was seen with an increasing number of previous treatments, although cognitive improvement was reported still relatively often after the fourth treatment. 
Table 3: Possible predictors of cognitive improvement at first follow-up

\begin{tabular}{|c|c|c|c|}
\hline Treatment category & $\begin{array}{c}\text { OR }(95 \% \mathrm{CI}) \\
\text { Univariate CC }\end{array}$ & $\begin{array}{c}\text { OR }(95 \% \mathrm{CI}) \\
\text { Univariate MI }\end{array}$ & $\begin{array}{c}\text { OR }(95 \% \mathrm{CI}) \\
\text { Multivariate MI }\end{array}$ \\
\hline AED & reference & reference & reference \\
\hline Benzodiazepines & $1.6(0.5-5.2)$ & $1.4(0.3-5.5)$ & reference \\
\hline Steroids & $3.7(1.2-11.4)$ & $3.3(1.1-10.4)$ & $2.5(1.1-5.7)$ \\
\hline Surgery & $3.3(0.2-61.7)$ & $2.8(0.1-54.5)$ & Reference \\
\hline IVIG & $1.7(0.4-7.5)$ & $1.4(0.3-7.0)$ & Reference \\
\hline Other & $1.1(0.2-7.5)$ & $1.3(0.2-6.5)$ & Reference \\
\hline \multicolumn{4}{|l|}{ Patient / treatment characteristics } \\
\hline Male gender & $0.9(0.4-2.0)$ & $0.9(0.4-2.0)$ & * \\
\hline Age at diagnosis & $1.01(0.99-1.02)$ & $1.01(0.99-1.02)$ & * \\
\hline Interval diagnosis - treatment & $0.98(0.95-1.00)$ & $0.98(0.95-1.01)$ & $0.99(0.96-1.02)$ \\
\hline Abnormal development before ESES onset & $0.6(0.3-1.3)$ & $0.6(0.3-1.2)$ & $0.6(0.3-1.4)$ \\
\hline MRI abnormalities & $1.4(0.6-3.0)$ & $1.3(0.6-2.9)$ & * \\
\hline Number of previous treatments & $0.78(0.64-0.95)$ & $0.77(0.63-0.95)$ & $0.81(0.63-1.03)$ \\
\hline
\end{tabular}

Legend: $95 \% \mathrm{Cl}=95 \%$ confidence interval, $\mathrm{CC}=$ complete case analysis, $\mathrm{MI}=$ analysis of multiple imputation dataset. $\mathrm{N}=119$ for complete case analysis, $\mathrm{n}=147$ for analysis after multiple imputation.

* $=$ Not included in multivariate model, because $p>0.20$ in univariate analysis

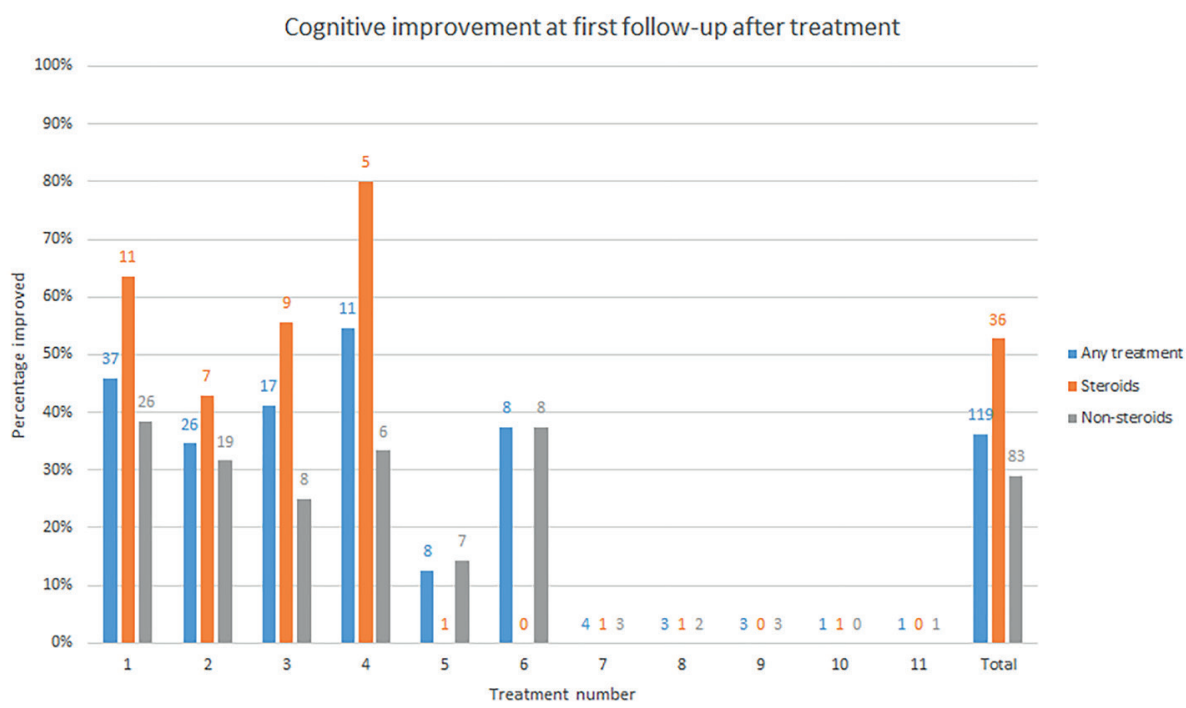

Figure 2: Cognitive improvement at first follow-up after treatment, related to the treatment number after the diagnosis of ESES.

The treatment number refers to the order in which treatments were given, i.e. the first treatment is treatment 1 , the fifth treatment is treatment 5 . The height of the bars reflects the percentage of treatments that was effective, the number on top of the bars reflects how many patients received this number of treatments, i.e. a fifth treatment was given in 8 patients, of which 7 had a non-steroid and 1 a steroid treatment and $12.5 \%$ of fifth treatments were successful. 
At last follow-up, higher age at diagnosis of ESES (OR 1.02; 95\% Cl $1.01-1.04$ ) showed an association with cognitive improvement, however this association was not significant in the multiple imputation dataset. (table 4)

\section{Secondary outcomes: behavior, seizure frequency and side effects}

Due to small numbers of patients in the different treatment categories we limited the analysis of secondary outcomes to a comparison of steroid versus non-steroid treatments. No significant differences were found in the effect of treatment on behavioral problems, seizure frequency and side effects between steroid treatments and non-steroid treatments.

Table 4: Possible predictors of cognitive improvement at last follow-up

\begin{tabular}{lcc}
\hline Treatment category & $\begin{array}{c}\text { OR }(95 \% \text { CI) } \\
\text { Univariate } \\
\text { CC }\end{array}$ & $\begin{array}{c}\text { OR (95\% CI) } \\
\text { Univariate } \\
\text { MI }\end{array}$ \\
\hline AED & reference & reference \\
Benzodiazepines & $0.7(0.2-2.0)$ & $0.6(0.2-2.1)$ \\
Steroids & $1.6(0.5-5.5)$ & $1.2(0.2-6.8)$ \\
Surgery & N/A (2/2 positive) & N/A (2/2 positive) \\
IVIG & $0.7(0.2-2.9)$ & $0.6(0.1-2.7)$ \\
Other & $0.8(0.2-3.5)$ & $0.7(0.1-3.5)$ \\
\hline Patient / treatment characteristics & & $1.0(0.4-2.4)$ \\
\hline Male gender & $0.9(0.4-2.2)$ & $1.02(0.99-1.04)$ \\
Age at diagnosis & $1.02(1.01-1.04)$ & $1.00(0.98-1.03)$ \\
Interval diagnosis - treatment & $1.00(0.98-1.02)$ & $0.7(0.2-2.2)$ \\
Abnormal development before ESES onset & $0.6(0.3-1.3)$ & $1.5(0.6-3.6)$ \\
MRI abnormalities & $1.6(0.7-3.6)$ & $0.90(0.75-1.07)$ \\
Number of previous treatments & $0.85(0.70-1.03)$ & \\
\hline
\end{tabular}

Legend:

$95 \% \mathrm{Cl}=95 \%$ confidence interval, $\mathrm{CC}=$ complete case analysis, $\mathrm{MI}=$ analysis of multiple imputation dataset. $\mathrm{N}=94$ for complete case analysis, $\mathrm{n}=147$ for analysis after multiple imputation.

Note: No multivariate analysis after multiple imputation is reported because only for age the $p$-value is $<0.2$ in univariate after multiple imputation.

\section{Discussion}

In this study we investigated the effect of treatment on - subjectively assessed - cognitive functioning, IQ test results and spike-wave index in 47 patients with ESES syndrome treated in our center. We found that, in general, cognitive improvement is reported after a minority of treatments (36\% at first follow-up and $45 \%$ at last follow-up). IQ results showed on average no clear change after treatment, while the EEG on average revealed 
a decrease in spike-wave index (-21 and -24\% at first and last follow-up respectively). Subjectively reported cognitive improvement was significantly related to SWI decrease but not to IQ changes. Corticosteroids were found to be significantly associated with improvement at first follow-up, as compared to all other treatment categories, while at last follow-up higher age at diagnosis was associated with improvement. The number of previous treatments seemed to be inversely correlated to improvement.

In clinical practice, subjective judgment of cognitive functioning and sleep EEG recordings are generally used to evaluate treatment effect, as these are more readily available than repeated neuropsychological assessments and not influenced by re-test bias. We investigated whether subjective cognitive improvement is accompanied by IQ improvement. Although an increase in IQ was seen in patients who were reported to have improved and a decrease in patients who did not improve, we found no significant association between subjective improvement and changes in IQ scores. A first possible explanation is that total IQ values are not a sensitive marker for cognitive functioning in children with ESES and can only be used to evaluate the course during long-term followup. Subjective improvement of functioning can be can be clinically very relevant, even if this does not lead to IQ improvement. Second, most children start treatment because of cognitive deterioration and in this setting an unchanged IQ after treatment (i.e. the child gains skills and remains stable compared to other children of the same age) can be seen as successful treatment and can be confirmed by parents as improvement in functioning. Another explanation may be that we analyzed IQ changes of only 33 treatments, because these were the only treatments that were evaluated with a neuropsychological test before any other treatment was started. This inherently resulted in reduced statistical power and may have caused selection bias. EEG evolution, however, showed a strong association with subjective cognitive improvement. Although SWI change was not correlated to IQ change at first follow-up, at last follow-up a correlation between SWI change and IQ change was seen that suggests that a decrease in SWI is correlated to a decrease in IQ. Although this correlation was statistically significant, we feel that due to the very small number of treatments $(n=7)$ in this analysis and the probable influence of selection bias, this finding should be interpreted with caution. Previous studies found that location, severity and duration of EEG abnormalities are correlated to cognitive performance and prognosis. $[6,21,22]$ Our study adds that SWI change is correlated to cognitive improvement, despite the fact that about half of the patients with cognitive improvement still had a SWI above 50\%.

We found that steroid treatment was significantly correlated to cognitive improvement at first follow-up, in contrast with all other treatment categories. At last follow-up this difference was not significant. The results of our treatment analysis are consistent with a 
Chapter 7. ESES: characteristics and treatment effect

recent pooled analysis of 575 ESES cases.[8] In this meta-analysis of the literature, steroid treatment was found to be associated with cognitive and EEG improvement compared to non-steroid medical treatment, although no directly comparing studies were included. The underlying mechanism explaining efficacy of steroids in patients with ESES is incompletely understood. Alterations in cytokine profiles have been found in children with ESES and an underlying inflammatory process has been suggested.[13,23] Corticosteroids may be of benefit by interfering in this pro-inflammatory state. A possible explanation for the non-significance at last follow-up could be that corticosteroids can lead to a temporary response by suppressing an inflammatory process, while after completing steroid pulses or after tapering oral steroids the inflammation and ESES related cognitive deficits reemerge. This is consistent with long-term relapses found in a previous cohort of ESES patients treated with corticosteroids.[14]

Our results have to be interpreted with some caution. First, the retrospective design of the study is inevitably associated with missing data. Especially, for many individual treatments neuropsychological follow-up was insufficient for a before-after comparison. This reflects that IQ measurements cannot reliably be repeated shortly after the first measurement, which limits their utility in the context of (the treatment of) ESES. Second, the lack of a placebo control group makes it impossible to distinguish true treatment effect from fluctuations in the natural course that are often seen in ESES patients. This may have influenced our comparison of the different treatment options for ESES. Third, the evaluation of non-steroid treatments was often earlier (after reaching the intended dosage) than for steroids (in many cases after completing the intended number of pulses). Therefore a slow response to an AED or benzodiazepine may have remained unnoticed at first follow-up, while a slow response to a steroid treatment was included. In addition, this longer interval allows more time for spontaneous recovery.

Despite these challenges, our study provides valuable information. To our knowledge, it is the largest single center study describing treatment effect in patients with ESES syndrome. Also, it is the first cohort study statistically comparing different methods (subjective assessment, neuropsychological assessment, EEG) used for the follow-up of treatment in a relatively large group of patients with ESES syndrome.

For definite conclusions regarding the treatment of ESES syndrome, adequately sized Randomized Controlled Treatments are required. EEGs and neuropsychological assessments need to be performed at pre-specified time-points. RESCUE ESES is a European multicenter randomized controlled trial, currently comparing treatment with corticosteroids to treatment with clobazam. 


\section{Acknowledgments}

This work was supported by grants from the Dutch Epilepsy Fund and the Wilhelmina Children's Hospital Research Fund. The funding sources had no direct involvement in this study.

\section{Competing interests}

Dr. van den Munckhof and Dr. Jansen report grants from the Dutch Epilepsy Fund (epilepsiefonds) and Wilhelmina Children's Hospital Research Fund and non-financial support by the European Clinical Research Infrastructure Network (ECRIN) during the conduct of the study. The other authors have no potential conflicts of interest to declare. 
Chapter 7. ESES: characteristics and treatment effect

\section{References}

1 Patry G, Lyagoubi S, Tassinari CA. Subclinical 'electrical status epilepticus'induced by sleep in children. Arch Neurol 1971;24:242-52. doi:http://dx.doi.org/10.1001/archneur.1971.00480330070006

2 Nickels K, Wirrell E. Electrical Status Epilepticus in Sleep. Semin Pediatr Neurol 2008;15:50-60. doi:10.1016/j.spen.2008.03.002

3 Commission on Classification and Terminology of the International League Against Epilepsy. Proposal for Revised Classification of Epilepsies and Epileptic Syndromes. Epilepsia 1989;30:389-99. doi:10.1111/j.1528-1157.1989.tb05316

4 Fernández IS, Chapman KE, Peters JM, et al. Continuous Spikes and Waves during Sleep: Electroclinical Presentation and Suggestions for Management. Epilepsy Res Treat 2013;2013:583531. doi:10.1155/2013/583531

5 Landau WM, Kleffner FR. Syndrome of acquired aphasia with convulsive disorder in children. Neurology 1957;7:1241, 8 pages following 1241. doi:10.1212/WNL.7.8.523

6 Scholtes FBJ, Hendriks MPH, Renier WO. Cognitive deterioration and electrical status epilepticus during slow sleep. Epilepsy Behav 2005;6:167-73. doi:10.1016/j.yebeh.2004.11.001

7 Kramer U, Sagi L, Goldberg-Stern H, et al. Clinical spectrum and medical treatment of children with electrical status epilepticus in sleep (ESES). Epilepsia 2009;50:1517-24. doi:10.1111 /j.1528-1167.2008.01891

8 van den Munckhof B, van Dee V, Sagi L, et al. Treatment of electrical status epilepticus in sleep: A pooled analysis of 575 cases. Epilepsia 2015;56:1738-46. doi:10.1111/epi.13128

9 Guzzetta F, Battaglia D, Veredice C, et al. Early thalamic injury associated with epilepsy and continuous spike-wave during slow sleep. Epilepsia 2005;46:889-900. doi:10.1111/j.1528-1167.2005.64504

Kersbergen KJ, De Vries LS, Leijten FSS, et al. Neonatal thalamic hemorrhage is strongly associated with electrical status epilepticus in slow wave sleep. Epilepsia 2013;54:733-40. doi:10.1111/epi.12131

11 Agarwal R, Kumar A, Tiwari VN, et al. Thalamic abnormalities in children with continuous spike-wave during slow-wave sleep: An F-18-fluorodeoxyglucose positron emission tomography perspective. Epilepsia 2016;57:263-71. doi:10.1111/epi.13278

12 Bartolini E, Falchi M, Zellini F, et al. The syndrome of polymicrogyria, thalamic hypoplasia, and epilepsy with CSWS. Neurology 2016;86:1250-9. doi:10.1212/WNL.0000000000002526

13 Van Den Munckhof B, De Vries EE, Braun KPJ, et al. Serum inflammatory mediators correlate with disease activity in electrical status epilepticus in sleep (ESES) syndrome. Epilepsia 2016;57:e45-50. doi:10.1111/epi.13274

14 Buzatu M, Bulteau C, Altuzarra C, et al. Corticosteroids as treatment of epileptic syndromes with continuous spike-waves during slow-wave sleep. Epilepsia 2009;50:68-72. doi:10.1111 /j.1528-1167.2009.02224

15 Bölsterli BK, Schmitt B, Bast T, et al. Impaired slow wave sleep downscaling in encephalopathy with status epilepticus during sleep (ESES). Clin Neurophysiol 2011;122:1779-87. doi:10.1016/j. clinph.2011.01.053

16 Bölsterli Heinzle BK, Fattinger S, Kurth S, et al. Spike wave location and density disturb sleep slow waves in patients with CSWS (continuous spike waves during sleep). Epilepsia 2014;55:584-91. doi:10.1111/epi.12576

17 Sánchez Fernández I, Chapman K, Peters JM, et al. Treatment for continuous spikes and waves during sleep (CSWS): Survey on treatment choices in North America. Epilepsia 2014;55:1099-108. doi:10.1111/epi.12678

18 Saltik S, Uluduz D, Cokar O, et al. A clinical and EEG study on idiopathic partial epilepsies with evolution into ESES spectrum disorders. Epilepsia 2005;46:524-33. doi:10.1111/j.0013-9580.2005.45004

19 Tassinari CA, Michelucci R, Forti A, et al. The electrical status epilepticus syndrome. Epilepsy Res Suppl 1992;6:111-5.http://www.ncbi.nlm.nih.gov/pubmed/1418468 
20 Massa R, de Saint-Martin a, Carcangiu R, et al. EEG criteria predictive of complicated evolution in idiopathic rolandic epilepsy. Neurology 2001;57:1071-9.http://www.ncbi.nlm.nih.gov/ pubmed/11571336

21 Pera MC, Brazzo D, Altieri N, et al. Long-term evolution of neuropsychological competences in encephalopathy with status epilepticus during sleep: A variable prognosis. Epilepsia 2013;54:77-85. doi:10.1111/epi.12313

22 Maltoni L, Posar A, Parmeggiani A. Long-term follow-up of cognitive functions in patients with continuous spike-waves during sleep (CSWS). Epilepsy Behav 2016;60:211-7. doi:10.1016/j. yebeh.2016.04.006

23 Lehtimaki KA, Liimatainen S, Peltola J, et al. The serum level of interleukin-6 in patients with intellectual disability and refractory epilepsy. Epilepsy Res 2011;95:184-7. doi:10.1016/j.eplepsyres.2011.03.004 [doi] 


\section{Supplementary material}

Supplementary table 1 Cognitive performance related to first treatment

\begin{tabular}{|c|c|c|c|c|c|c|}
\hline \multirow[t]{2}{*}{ Treatment category } & \multirow{2}{*}{$\begin{array}{c}\begin{array}{c}\text { before } \\
\text { treatment }\end{array} \\
\text { mean / median } \\
\text { IQ }\end{array}$} & \multicolumn{3}{|c|}{ Evaluation before subsequent treatment ${ }^{*}$} & \multicolumn{2}{|c|}{$\begin{array}{l}\text { Regardless of other } \\
\text { treatments** }\end{array}$} \\
\hline & & $\begin{array}{c}\text { cognitive } \\
\text { improvement } \\
\text { at first FU } \\
\text { (subjective) }\end{array}$ & $\begin{array}{c}\text { cognitive } \\
\text { improvement } \\
\text { at last FU } \\
\text { (subjective) }\end{array}$ & $\begin{array}{c}\text { First FU mean } \\
\text { I median } \\
\text { delta IQ*** }\end{array}$ & $\begin{array}{l}\text { first FU mean } \\
\text { I median } \\
\text { delta IQ }\end{array}$ & $\begin{array}{l}\text { last FU mean } \\
\text { I median } \\
\text { delta IQ }\end{array}$ \\
\hline $\operatorname{AED}(n=9)$ & $72 / 71(n=6)$ & $3 / 7(43 \%)$ & $3 / 6(50 \%)$ & $+4 /+4(n=2)$ & $\begin{array}{c}+2 /-2.5 \\
(n=6)\end{array}$ & $\begin{array}{c}-15 /-9 \\
(n=5)\end{array}$ \\
\hline benzodiazepines ( $n=21)$ & $\begin{array}{l}72 / 75 \\
(n=16)\end{array}$ & $7 / 19(37 \%)$ & $8 / 16(50 \%)$ & $-5 /-3(n=8)$ & $\begin{array}{l}-5 /-6 \\
(n=12))\end{array}$ & $\begin{array}{l}-4 /-4 \\
(n=4)\end{array}$ \\
\hline Steroids ( $n=14$ ) & $\begin{array}{l}84 / 87 \\
(n=12)\end{array}$ & $7 / 11(64 \%)$ & $5 / 6(83 \%)$ & $\begin{array}{l}+1 /+3 \\
(n=11)\end{array}$ & $\begin{array}{c}+2 /+3.5 \\
(n=10)\end{array}$ & $\begin{array}{l}-4 /-1 \\
(n=7)\end{array}$ \\
\hline Other $(n=1)$ & N/A & N/A & N/A & N/A & N/A & $N / A$ \\
\hline All $(n=45)$ & $\begin{array}{l}76 / 77 \\
(n=34)\end{array}$ & $17 / 37(46 \%)$ & $16 / 28(57 \%)$ & $\begin{array}{l}-1 / 0 \\
(n=21)\end{array}$ & $\begin{array}{l}-1 /-1 \\
(n=28)\end{array}$ & $\begin{array}{l}-7.5 /-6 \\
(n=16)\end{array}$ \\
\hline
\end{tabular}

Notes:

-Mean / median duration to first follow-up of subjective cognitive functioning was 1.3 / 1.0 (SD 0.9) months for AED, 1.9 / 1.0 (SD 2.4) months for benzodiazepines, 9.3 / 6.0 (SD 8.3) months for steroid treatments and 4.1 / 2.0 months (SD 6.1 months) for all treatments.

-Mean / median time to last follow-up was 3.8 / 2.5 (SD 4.8) months for AED, 12.5 / 7.0 (SD 15.6) months for benzodiazepines, 21.9 / 14.0 (SD13.4) months for steroid treatments and 13.8/9.0 (SD 14,6) months for all treatments. All analyses presented here are based on complete cases.

- Mean / median time to first FU IQ regardless of other treatments: 8.2 / 8.0 (SD 3.9) months for AED, 8.4 / 8.0 (SD 5.5) months for benzodiazepines, 6.7 / 6.0 (SD 4.6) months for steroids and 7.7 / 7.0 (SD4.7) months for all treatments.

- Mean time to last FU IQ regardless of other treatments: 42.5 / 35.0 (SD 31.1) months for AED, 22.4 / 27.0 (SD 13.5) months for benzodiazepines, 34.9 / 26.0 (SD 25.0) months for steroids and 34.8/ 29.0 (SD25.6) months for all treatments.

* Evaluation before subsequent treatment means that the first and last follow-up were collected before a new treatment was started.

** Regardless of other treatments means that these data were gathered without taking subsequent treatments into account. So even if a new treatment was started after the first treatment, the follow-up is still considered as follow-up of the first treatment choice.

*** Insufficient data available for calculation of delta $\mathrm{IQ}$ at last follow-up of first treatment.

Supplementary table 2 Spike-wave indices related to first treatment (follow-up before any other treatment was started)

\begin{tabular}{|c|c|c|c|}
\hline Treatment category & before treatment & First follow-up & Last follow-up \\
\hline & mean / median SWI & $\begin{array}{c}\text { Mean / median SWI change } \\
\text { (first after treatment - before) }\end{array}$ & $\begin{array}{l}\text { mean / median SWI change } \\
\text { (last after treatment - before) }\end{array}$ \\
\hline AED & $\begin{array}{c}88 \% / 85 \% \\
(n=7)\end{array}$ & $\begin{array}{c}+3 \% /+3 \% \\
(n=2, \text { mean FU } 2.3 \text { months })\end{array}$ & $\begin{array}{c}+6 \% /+6 \% \\
(n=2, \text { mean FU } 9 \text { months })\end{array}$ \\
\hline benzodiazepines & $\begin{array}{c}74 \% / 83 \% \\
(n=21)\end{array}$ & $\begin{array}{c}-26 \% /-9 \% \\
(n=17, \text { mean FU } 5 \text { months })\end{array}$ & $\begin{array}{c}-17 \% /-4 \% \\
(n=8, \text { mean FU } 16 \text { months })\end{array}$ \\
\hline Steroids & $\begin{array}{c}92 \% / 96 \% \\
(n=11)\end{array}$ & $\begin{array}{c}-50 \% /-56 \% \\
(n=10, \text { mean FU } 9.3 \text { months })\end{array}$ & $\begin{array}{c}-54 \% /-58 \% \\
(n=4, \text { mean FU } 26 \text { months })\end{array}$ \\
\hline Other & $\begin{array}{r}56 \% \\
(n=1)\end{array}$ & $\begin{array}{c}-17 \% \\
(n=1, \text { FU } 4 \text { months })\end{array}$ & $\begin{array}{c}-53 \% \\
(n=1, \text { FU } 17 \text { months })\end{array}$ \\
\hline All & $\begin{array}{c}81 \% / 85 \% \\
(n=40)\end{array}$ & $\begin{array}{c}-32 \% /-23 \% \\
(n=30, \text { mean FU } 6.3 \text { months })\end{array}$ & $\begin{array}{c}-26 \% /-21 \% \\
(n=15, \text { mean FU }=18 \text { months })\end{array}$ \\
\hline
\end{tabular}

Note:

All analyses presented here are based on complete cases. 
Supplementary table 3. Treatment effect in relation to cognitive performance and SWI of all treatments (regardless of number of previous treatments)

\begin{tabular}{|c|c|c|c|c|c|}
\hline \multirow[t]{2}{*}{ Treatment category } & \multirow{2}{*}{$\begin{array}{c}\text { before treatment } \\
\text { mean / median } \\
\text { SWI }\end{array}$} & \multicolumn{2}{|c|}{ First follow-up } & \multicolumn{2}{|c|}{ Last follow-up } \\
\hline & & $\begin{array}{c}\text { cognitive } \\
\text { improvement }\end{array}$ & $\begin{array}{c}\text { mean / median SWI } \\
\text { change } \\
\text { (first-before) }\end{array}$ & $\begin{array}{c}\text { cognitive } \\
\text { improvement }\end{array}$ & $\begin{array}{c}\text { Mean / median } \\
\text { SWI change } \\
\text { (last-before) }\end{array}$ \\
\hline $\begin{array}{l}\text { AED } \\
(n=35)\end{array}$ & $\begin{array}{c}86 \% / 91 \% \\
(n=20)\end{array}$ & $\begin{array}{c}6 / 26(23 \%) \\
\text { (FU 3.9 months) }\end{array}$ & $\begin{array}{c}-16 \% /-4 \% \\
\text { (n=10, } \\
\text { FU } 8.8 \text { months) }\end{array}$ & $\begin{array}{l}11 / 24 \\
(46 \%)\end{array}$ & $\begin{array}{c}-33 \% /-28 \% \\
(n=7, \text { mean } \\
\text { FU } 21 \text { months) }\end{array}$ \\
\hline $\begin{array}{l}\text { Benzodiazepines } \\
(\mathrm{n}=39)\end{array}$ & $\begin{array}{c}74 \% / 82 \% \\
(n=32)\end{array}$ & $\begin{array}{l}10 / 31 \text { (32\%) } \\
\text { (FU } 1.7 \text { months) }\end{array}$ & $\begin{array}{c}-27 \% /-15 \% \\
\text { (n= }=24, \text { mean FU } 4.2 \\
\text { months) }\end{array}$ & $\begin{array}{l}10 / 28 \\
(36 \%)\end{array}$ & $\begin{array}{l}-17 \% /-17 \% \\
\text { ( } n=12, \text { mean } \\
\text { FU } 16 \text { months) }\end{array}$ \\
\hline $\begin{array}{l}\text { Steroids } \\
(n=43)\end{array}$ & $\begin{array}{c}80 \% / 92 \% \\
(n=34)\end{array}$ & $\begin{array}{l}19 / 36 \text { (53\%) } \\
\text { (FU } 9.3 \text { months) }\end{array}$ & $\begin{array}{c}-26 \% /-15 \% \\
\text { (n=26, mean } \\
\text { FU } 11 \text { months) }\end{array}$ & $\begin{array}{l}11 / 19 \\
(58 \%)\end{array}$ & $\begin{array}{c}-57 \% /-68 \% \\
\text { (n=8, mean } \\
\text { FU } 27 \text { months) }\end{array}$ \\
\hline $\begin{array}{l}\text { Surgery } \\
(n=2)\end{array}$ & $\begin{array}{l}100 \% \\
(n=1)\end{array}$ & $\begin{array}{l}1 / 2 \\
(50 \%)\end{array}$ & $\begin{array}{c}-100 \% \\
\text { (n=1, FU } 3.5 \text { months) }\end{array}$ & $\begin{array}{l}2 / 2 \\
(100 \%)\end{array}$ & $\begin{array}{c}-100 \% \\
(n=1, \text { FU } 4.0 \text { months })\end{array}$ \\
\hline $\begin{array}{l}\text { IVIG } \\
(n=12)\end{array}$ & $\begin{array}{c}84 \% / 99 \% \\
(n=8)\end{array}$ & $\begin{array}{l}4 / 12 \\
(33 \%)\end{array}$ & $\begin{array}{c}-1 \% / 0 \% \\
\text { ( } \mathrm{n}=7, \text { mean } \\
\text { FU } 4.5 \text { months) }\end{array}$ & $\begin{array}{l}4 / 11 \\
(36 \%)\end{array}$ & $\begin{array}{c}+1 \% / 0 \% \\
\text { (n=3, mean } \\
\text { FU } 15 \text { months) }\end{array}$ \\
\hline $\begin{array}{l}\text { Other } \\
(n=16)\end{array}$ & $\begin{array}{c}63 \% / 63 \% \\
(n=12)\end{array}$ & $\begin{array}{l}3 / 12 \\
(25 \%)\end{array}$ & $\begin{array}{c}-7 \% /-1 \% \\
\text { ( } n=6, \text { mean } \\
\text { FU } 3 \text { months) }\end{array}$ & $\begin{array}{l}4 / 10 \\
(40 \%)\end{array}$ & $\begin{array}{l}-18.3 \% /-2 \% \\
\text { ( }=3, \text { mean } \\
\text { FU } 13 \text { months) }\end{array}$ \\
\hline $\begin{array}{l}\text { All treatments } \\
(n=147)\end{array}$ & $\begin{array}{c}78 \% / 85 \% \\
(n=107)\end{array}$ & $\begin{array}{l}43 / 119 \\
(36 \%)\end{array}$ & $\begin{array}{l}-21 \% /-5 \% \\
\text { (n=74, mean } \\
\text { FU7 months) }\end{array}$ & $\begin{array}{l}42 / 94 \\
(45 \%)\end{array}$ & $\begin{array}{c}-31 \% /-24 \% \\
\text { ( } n=34 \text {, mean } \\
F U=18 \text { months) }\end{array}$ \\
\hline
\end{tabular}

Notes:

- Mean time to first cognitive follow-up was 4.8 months for all treatments, 3.9 months for AED, 1.7 months for benzodiazepines, 9.2 months for steroids, 0.5 months for surgery, 3.0 months for IVIG and 4.1 for other treatments. Mean time to last cognitive follow-up was 13.8 months for all treatments, 12.6 months for AED, 11.9 for benzodiazepines, 20.7 for steroids, 18.0 for surgery, 12.2 for IVIG and 9.1 for other treatments.

- Mean time to first EEG follow-up was 7 months for all treatments, 9 months for AED, 4 months for benzodiazepines, 11 months for steroids, 4 months for surgery, 5 months for IVIG and 3 months for other treatments. Mean time to last EEG follow-up was 18 months for all treatments, 21 months for AED, 16 months for benzodiazepines, 27 months for steroids, 4 months for surgery, 15 months for IVIG and 13 months for other treatments. 

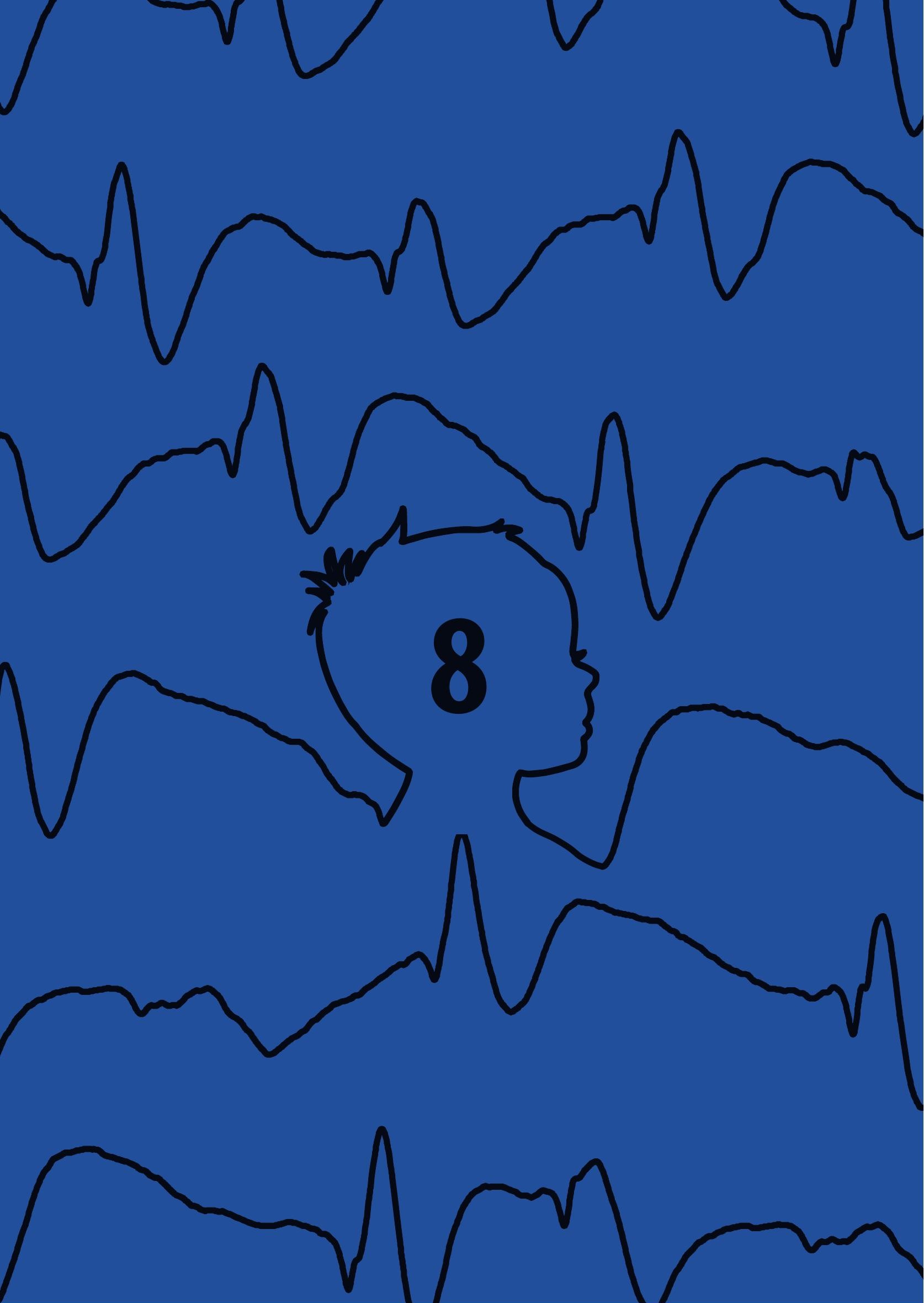


\section{Chapter 8}

\section{Corticosteroids versus}

clobazam in epileptic encephalopathy with ESES:

a European multicenter randomized controlled clinical trial (RESCUE ESES*)

* Randomized European trial of Steroids versus Clobazam Usage for Encephalopathy with Electrical Status Epilepticus in Sleep

Bart van den Munckhof

Alexis Arzimanoglou

Emilio Perucca

Heleen C. van Teeseling

Frans S.S. Leijten

Kees P.J. Braun

Floor E. Jansen

on behalf of the RESCUE ESES study group 


\section{Abstract}

Background: Epileptic encephalopathy with electrical status epilepticus in sleep (ESES) is an epilepsy syndrome occurring almost exclusively in children, usually at an age between 4 and 12 years. It is characterized by abundant sleep-induced epileptic activity in the electroencephalogram (EEG) and by acquired cognitive and behavioural deficits. The goal of treatment is to prevent further decline or even improve cognitive functioning. Based on mostly small and retrospective studies, corticosteroids and clobazam are regarded by many clinicians as the most effective pharmacological treatments. This European multicentre randomized controlled trial is designed to compare the effects of corticosteroids and clobazam on cognitive functioning after 6 months. Secondary outcomes include cognitive functioning after 18 months, EEG abnormalities in sleep, safety and tolerability, and seizure frequency. We also aimed at investigating whether treatment response in epileptic encephalopathy with ESES can be predicted by measurement of inflammatory mediators and auto-antibodies in serum.

Methods: The pragmatic study will be performed in centres with expertise in the treatment of rare paediatric epilepsy syndromes across Europe. 130 patients, 2 to 12 years of age, with epileptic encephalopathy with ESES will be enrolled and randomized in a 1:1 ratio to receive either corticosteroids (monthly intravenous methylprednisolone pulses or daily oral prednisolone) or oral clobazam for 6 months according to an open-label parallelgroup design. Follow-up visits with clinical assessment, EEGs and neuropsychological testing are scheduled for up to 18 months. Blood samples for cytokine and auto-antibody testing are obtained before treatment and after 8 months of treatment.

Discussion: The treatment of epileptic encephalopathy with ESES aims at improving cognitive outcome. This randomized controlled study will compare the most frequently used treatments, i.e. corticosteroids and clobazam. If the study proves superiority of one treatment over the other or identifies biomarkers of treatment response, results will guide clinicians in the early treatment of this severe epilepsy syndrome.

Trial registration: ISRCTN, ISRCTN42686094, registered 24 May 2013, http://www.isrctn. com/ISRCTN42686094 


\section{Background}

Electrical status epilepticus in sleep (ESES) was initially described as a subclinical electroencephalographic (EEG) pattern of sleep-induced spikes and waves (SWs) in children, occupying 85-100\% of slow wave sleep.' When bilateral ESES is associated with cognitive decline or behavioural disturbances, a diagnosis of encephalopathy with ESES can be made. The cognitive deficits are global (often referred to as continuous spikes and waves syndrome, CSWS) or confined to a specific cognitive domain (e.g. acquired aphasia or auditory agnosia in children with Landau-Kleffner Syndrome, LKS). 2,3 These phenotypes are referred to as "typical ESES patients" 4,5 The exact incidence of the epileptic encephalopathy with ESES is unknown, but it is estimated to constitute $0.2-1.9 \%$ of paediatric epilepsy cases., ${ }^{5,6}$

ESES spectrum variants, are increasingly recognized and include children with ESES and developmental delay but without arrest or regression of development ${ }^{7}$. Also, the spike wave index (SWI) threshold to diagnose ESES can be flexible, and can be $<85 \%$, provided that the main feature of epileptic encephalopathy with ESES, i.e. occurrence of cognitive and behavioral deterioration associated with a striking enhancement of epileptic activity during NREM sleep, is demonstrated. ${ }^{8}$ How ESES causes cognitive deficits and which factors determine cognitive outcome remains largely unknown. It has been suggested that ESES disrupts synaptic homeostasis, i.e. the balanced synaptic potentiation during daytime and synaptic downscaling in sleep, leading to an inefficient cerebral network. ${ }^{9}$ Epileptic encephalopathy with ESES has been reported in patients with structural abnormalities (e.g. perinatal thalamic injury) ${ }^{10,11}$, genetic disorders (e.g. a GRIN2A mutation $)^{12,13}$, though in about half of the cases no clear cause is identified. Evidence is accumulating for a role of the immune-system in patients with epilepsy. ${ }^{14}$ In children with Landau-Kleffner, autoantibodies to central and peripheral myelin, cell nucleus and blood vessels, in sera and cerebrospinal fluid have been found. ${ }^{15}$ In addition, in a previous study we found that several cytokines were significantly higher in blood samples of patients with encephalopathy with ESES compared with healthy controls. ${ }^{16}$

While the EEG-abnormalities characteristic of ESES generally resolve spontaneously during puberty, cognitive deficits often remain. ${ }^{17,18}$ Treatment during the active ESES phase may improve EEG abnormalities and daily functioning. ${ }^{19,20}$ In fact, successful early treatment (and thereby a shorter ESES duration) is associated with improvement of long-term cognitive outcome. ${ }^{18,21}$ However, the management of encephalopathy with ESES is often challenging and there is no consensus on which is the best initial treatment.2 Moreover, no adequately powered randomised controlled trials have been performed in children with encephalopathy with ESES. 
Chapter 8. Corticosteroids vs. clobazam for ESES (RESCUE ESES)

We recently performed a pooled analysis of 950 treatments in 575 cases with encephalopathy with ESES, reported in 112 articles. Conventional anti-epileptic drugs, often prescribed to control concomitantly occurring epileptic seizures, were generally not very effective in treating ESES and its associated cognitive deficits (improvement of EEG or cognition in 49\%). Benzodiazepines and corticosteroids seemed to be more effective, with improvement in $68 \%$ and $81 \%$ of cases, respectively. However, in a subgroup analysis that included only consecutively assessed patients, a smaller proportion showed any improvement (34\% with conventional anti-epileptic drugs, 59\% with benzodiazepines and $75 \%$ with corticosteroids). A subgroup of patients with a focal structural abnormality benefitted from epilepsy surgery. These results have to be interpreted with caution because most included studies were small, retrospective and heterogeneous, and sideeffects were not considered..$^{19}$

Review articles on the treatment of encephalopathy with ESES concluded that no standard approach exists and mentioned high-dose benzodiazepines and (cortico)steroids as preferred options. ${ }^{5,6,23}$ Among benzodiazepines, clobazam is often considered the most suitable although sedation and agitation are frequent side-effects. Corticosteroids have been given in variable dosing regimens, and concerns for side-effects such as weight gain and increased blood glucose may be a reason to consider them as a second-line option. All authors emphasize that the evidence guiding the treatment of encephalopathy with ESES is unsatisfactory and therefore a randomised controlled trial is urgently needed.

We hypothesise that, in comparison to treatment with clobazam, corticosteroids are more effective in improving cognitive performance and EEG abnormalities in children with encephalopathy with ESES. Based on previous studies, the difference in percentage of responders is estimated at around $25 \% .^{19}$ The use of corticosteroids may be associated with more frequent or more severe side-effects than clobazam.

In a multicentre randomized controlled trial with 1:1 allocation to corticosteroids and clobazam, we aim to assess whether one of the treatments is superior to the other. The following study objectives and hypotheses are addressed in a population of recently diagnosed patients with encephalopathy with ESES:

\section{Primary study objective:}

1. to compare the effects of treatment with corticosteroids or clobazam on cognition at 6 months after start of the treatment. 


\section{Secondary study objectives:}

2. to compare the effects of treatment with corticosteroids or clobazam on sleep-induced epileptiform activity, measured as the spike-wave index (SWI), in the patients'sleep EEG.

3. to compare the effects of treatment with corticosteroids or clobazam on the frequency of any concomitant seizures.

4. to compare the side effects and tolerability of corticosteroids and clobazam.

5. to compare the effects of treatment with corticosteroids and clobazam on subjective daily functioning, as measured with a visual analogue score (VAS).

6. to assess demographic and disease-related biomarkers, including immunological biomarkers, as potential predictors of disease activity and response to treatment with corticosteroids or clobazam.

The study is conducted as a randomized open-label parallel-group controlled trial with $1: 1$ treatment allocation to clobazam and corticosteroid treatment arms and is aimed at proving superiority of one treatment over the other. The trial also uses a pragmatic approach, whereby participating investigators will be allowed to apply, within predetermined limits, the dosing schedules which they consider best according to their judgment and patient response. The same flexibility will also apply to the option of using i.v. versus oral steroids, which will be left to the discretion of the treating physicians.

\section{Methods}

\section{Study setting}

Study preparations have been initiated in 22 centres with expertise in the treatment of rare paediatric epilepsy syndromes across 12 European countries.

\section{Study population}

The study will include 130 patients with encephalopathy with ESES, with typical or atypical presentation and symptoms, according to the following eligibility criteria. The overall duration of follow-up will be 18 months. 
Chapter 8. Corticosteroids vs. clobazam for ESES (RESCUE ESES)

Inclusion criteria:

- Age at inclusion: 2 up to <12 years;

- A diagnosis within six months prior to enrolment (preferably as close to enrolment as possible) of either:

- $\quad$ Bilateral sleep-induced epileptiform activity with an SWI >85\% in non-REM sleep and developmental delay, arrest, or regression ("typical epileptic encephalopathy with ESES");

- Arrest or regression of development and bilateral sleep-induced epileptiform activity with an SWI $>50 \%$, or unilateral sleep-induced epileptiform activity with an SWI $>85 \%$ in non-REM sleep ("atypical epileptic encephalopathy with ESES");

- Regression of development and unilateral epileptiform activity with an SWI $>50 \%$ in non-REM sleep ("atypical epileptic encephalopathy with ESES");

- No previous treatment with either corticosteroids or clobazam;

- No current treatment, nor treatment in the previous three months, with carbamazepine, oxcarbazepine, vigabatrin, tiagabine, gabapentin and pregabalin; These drugs potentially increase the SWI during sleep and may cause an electrographic pattern fulfilling the criteria for ESES and subsequently worsen outcome in children with epileptic encephalopathy with ESES and may thereby influence treatment results. Therefore inclusion of such cases with possible "treatment-induced ESES" is not desirable.

- Written informed consent by parents / legal representatives

Exclusion criteria:

- Patients with a SWI during wakefulness of $>50 \%$

- Any condition that, in the investigator's judgement, contra-indicates the use of corticosteroids or clobazam, such as acute or chronic infectious disease (e.g. tuberculosis, HIV), immunodeficiency, severe osteopenia/osteoporosis, diabetes mellitus, Cushing syndrome, severe respiratory insufficiency, severe liver failure or gastrointestinal ulcer.

\section{Informed consent}

Parents / legal representatives are informed by the treating doctor about the background, study design and study procedures. A patient information leaflet is provided to all parents / legal representatives and a leaflet version for the children above 6 years of age is available according to their abilities to understand the content. Parents / legal representatives / patients have at least 7 days to consider participation and their questions will be answered. Informed consent forms with approval for participation in the study, including the collection of blood samples and the storage of study data for a fixed period (depending 
on the standard per country, 15 years for the Netherlands), will be signed and filed. Study participants may withdraw from the trial anytime during the conduct of the study.

\section{Treatment}

Patients will be enrolled by their treating physician / study doctor, who has no direct insight in the allocation mechanism of the randomization module. Treatment is allocated according to an automatic online randomization module with block randomization stratified for centre to the two treatment regimens (1:1 ratio). The randomization module was programmed by a data-manager. While the patients and treating physicians are not blinded for treatment allocation (for practical reasons and because the two treatments require different monitoring), the neuropsychological assessment and EEG assessment will be performed by personnel blinded for treatment.

- Clobazam will be administered orally and increased to a dose of $0.5 \mathrm{mg} / \mathrm{kg} / \mathrm{day}$ within 2 weeks. If well tolerated, dosage may be increased up to $1.2 \mathrm{mg} / \mathrm{kg} /$ day (given once daily, in the evening). Treatment with a dosage of at least $0.5 \mathrm{mg} / \mathrm{kg} /$ day will be continued for 6 months and thereafter will be either continued or tapered according to the treating physician's preference. Clobazam is defined by active substance for this study. It is prescribed as tablets and brand names include Frisium, Onfi, Tapclob as well as generic products.

- Corticosteroids: Either intravenous methylprednisolone or oral prednisolone will be used, depending on local experience and preference.

Intravenous methylprednisolone will be given as monthly pulses. A dosage of $20 \mathrm{mg} / \mathrm{kg}$ will be given over 30 min once a day for 3 consecutive days, every four weeks, for a total period of 6 months with the intention to stop thereafter.

Oral prednisolone will be administered at an initial dosage of $2 \mathrm{mg} / \mathrm{kg} /$ day (not exceeding $60 \mathrm{mg} /$ day) for one month, followed in the $2^{\text {nd }}$ through $6^{\text {th }}$ month by a dosage between 1 and 2 mg/kg/day (not exceeding 60 mg/day), according to the treating physician's judgment. Thereafter, prednisolone will be either continued or tapered according to the treating physician's preference.

Treatment will be continued for at least 6 months, unless informed consent is withdrawn, the patient develops intolerable adverse effects, or further cognitive regression occurs, requiring an alternative intervention in the opinion of the treating physician. If cognitive regression is observed to continue after 3 months of treatment, according to the impression of the parents or physician, switching to the other treatment arm is allowed. In patients requiring switching to an alternative treatment, an EEG will be obtained before switching. 
Drug adherence to oral treatment (oral clobazam or oral prednisolone) will be optimized and monitored by instructing patients to bring their empty packages of study medication to the hospital at every scheduled visit, together with completed drug intake diaries. In addition, for patients treated with clobazam, plasma levels will be measured in blood samples collected between 1 and 3 months after enrolment and between 3 and 6 months after enrolment. For intravenous methylprednisolone, adherence will be confirmed by recording drug administration at times of hospital admission for the monthly pulses.

Concomitant medication will be allowed as long as the patient fulfils the criteria for inclusion. However, changes in concomitant medications are discouraged during the first 6 months. After assessment of the primary endpoint at 6 months, subsequent treatment strategies will be left to the clinical judgment of the treating physician.

\section{Outcomes}

The co-primary outcome measures at six months will be cognitive functioning, assessed with a full neuropsychological assessment (NPA):

- Intelligence quotient (IQ), or developmental quotient (DQ), compared to baseline IQ / DQ. Improvement is defined as an increase by 10 IQ / DQ points.

- Cognitive sumscore (as defined below). Improvement is defined as statistically significant when improved by at least $75 \%$ of the standard deviation (SD).

\section{Secondary outcome measures at 6 and 18 months will include:}

- changes in individual absolute test results, and IQ / DQ scores, compared to baseline;

- changes in spike wave index (SWI) during non-REM sleep, compared with baseline SWI;

- changes in seizure frequency assessed for all reported seizure types combined, with improvement being defined as at least 50\% decrease as compared with baseline;

- changes in subjective global daily functioning assessed with a visual analogue score (VAS) of -5 to +5 as compared with baseline;

- safety and tolerability, as assessed by the occurrence of adverse events;

- change in inflammatory markers post treatment as compared to levels prior to treatment

- Identification of auto-antibodies as potential biomarkers of disease severity (TIQ, presence of developmental regression, arrest or delay and SWI at baseline) and treatment efficacy.

\section{Data collection}

All patient data for the study will be recorded in the online case report form with reference to the patient study number, in compliance with Good Clinical Practice guidelines. Data will be stored in a secure data platform, managed by an independent data-manager. 
Baseline data ( $t=0$ months) will be collected before treatment initiation and include patient demographics, date of ESES diagnosis, onset of ESES, seizure type(s), anti-epileptic drug history, detailed history of psychomotor development and behaviour, estimated age at onset of developmental arrest or regression, impression of global functioning assessed with VAS score (-5 to 5) and neurological examination. Ancillary investigations related to aetiology will be reassessed or scheduled (if not yet performed) and will include a cerebral MRI (dedicated epilepsy protocol) and genetic tests if no aetiology is known (array CGH, mutation analysis including GRIN2A gene). Metabolic screening and a cerebrospinal fluid tap will be performed if considered indicated.

\section{Sleep EEG at baseline and after 1, 3, 6 and 18 months}

The diagnosis of encephalopathy with ESES will have to be confirmed by whole night EEG recording prior to randomization. Depending on the logistics of the participating centres, either sleep-deprived EEGs of at least one hour or whole-night recordings will be considered adequate for follow-up assessments. Technical requirements for the EEG recordings are specified in an appendix to the study protocol. Clinical neurophysiologists will be blinded to the type of treatment.

For each EEG, the spike wave index (SWI) will be calculated in an epoch of 10 minutes (600 s) duration, starting 5 minutes after alpha attenuation or after sleep had clinically commenced. The number of seconds containing epileptiform discharges is divided by the total number of seconds in the epoch (600) and multiplied by 100 to reflect the SWI as a percentage.

\section{Neuropsychological assessment at baseline, after 6 months and after 18 months}

Depending on the age and abilities of the patient, tests will be selected from a fixed battery covering the major domains of cognition (intelligence, language, memory, attention, visuospatial functions, executive functions, as specified in an appendix of the study protocol). Administration and scoring will be conducted according to the test manuals. Individual raw test scores at baseline and at follow-up will be transformed into $z$-scores, based on the mean and SD of standard scores. As a measure of overall cognitive functioning, a cognitive sum score will be calculated, representing the mean z-score over the 6 domains. Neuropsychologists will be blinded to treatment. 


\section{Cytokine profiles and auto-antibodies at baseline and 8 months after start of treatment ( 2 months after withdrawal of study steroids, to limit the influence of a possible decrease in levels caused solely by treatment)}

A snapshot of around 100 cytokines will be analysed (Luminex, X-map technology) in serum of the study participants, a) at randomisation and b) 8 months after start of treatment (2 months after withdrawal of corticosteroids). Screening for auto-antibodies will be performed using rat brain immunohistochemistry, optimized for extracellular antigens, like NmDAR, AMPaR, GAD, GABABR, LGI1, Caspr2.24 In addition, all samples will be tested by in house cell based assays for anti-NMDAR en anti-GlyR antibodies. Samples with positive staining, but no known antibodies, will be tested by immunocytochemistry using live hippocampal neurons. If positive, immunoprecipitation will be the first step towards antigen discovery.

\section{Data collection during follow-up}

Epilepsy characteristics and information on neurodevelopment, other medical history, neurological examination, concomitant medications, possible treatment emergent adverse events, and sleep EEGs will be collected 1, 3, 6 and 18 months after start of treatment. Neuropsychological assessments will be repeated only after 6 and 18 months to minimize re-test bias. After three months a brief assessment of cognitive performance is performed by the clinician to detect possible ongoing regression that may warrant a change of treatment. In the corticosteroid treatment group, additional safety and tolerability assessments will include monitoring of blood pressure, glucose and protein in urine and weight monitoring once weekly plus additionally at any hospital visits (including admissions for methylprednisolone pulses, when applicable). In case of stress, high fever or illness managements in these situations will be left to treating physician and details will be recorded in eCRF.

\section{Participant timeline}

A schedule of enrolment, interventions and assessments for participants is provided in table 1.

\section{Statistical analysis}

The primary analyses will be performed according to the intention-to-treat principle. We will also perform (secondary) analyses in patients who have completed their assigned treatment for the period of 6 months.

Continuous outcomes will be presented with means and 95\% confidence intervals. These outcomes will primarily be compared between the two treatment groups using a t-test or Mann-Whitney U-test depending on the distribution of the data (normal vs not normal). 
Categorical outcomes will be presented as proportions with 95\% confidence intervals. These outcomes will primarily be compared between the two treatment groups using the Fisher's Exact test. Rates of (serious) adverse events will be compared in terms of risk ratios with corresponding 95\% confidence intervals. We will also identify the proportion of patients that continue on the initially allocated treatment throughout the entire study period, and analyse possible predictors for discontinuation.

Table 1. Schedule of enrolment, interventions and assessments for participants

\begin{tabular}{|c|c|c|c|c|c|c|c|}
\hline \multirow[b]{3}{*}{ Timepoint* } & \multicolumn{7}{|c|}{ Study period } \\
\hline & \multirow{2}{*}{$\frac{\text { Enrolment }}{-\mathrm{t}_{1}}$} & \multirow{2}{*}{$\frac{\text { Randomization }}{t_{0}}$} & \multicolumn{4}{|c|}{ Post-allocation } & \multirow{2}{*}{$\frac{\text { Close-out }}{T_{18}}$} \\
\hline & & & $t_{1}$ & $\mathrm{t}_{3}$ & $t_{6}$ & $t_{8}$ & \\
\hline \multicolumn{8}{|l|}{ Enrolment } \\
\hline Eligibility screen & $x$ & & & & & & \\
\hline Informed Consent & $x$ & & & & & & \\
\hline Allocation & & $x$ & & & & & \\
\hline \multicolumn{8}{|l|}{ Interventions } \\
\hline Clobazam** & & 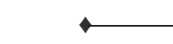 & & & $\rightarrow$ & & \\
\hline Steroids** & & $\vdash$ & & & $\rightarrow$ & & \\
\hline \multicolumn{8}{|l|}{ Assessments } \\
\hline $\begin{array}{l}\text { Epilepsy characteristics and information on } \\
\text { neurodevelopment, other medical history, } \\
\text { neurological examination, concomitant } \\
\text { medications }\end{array}$ & $x$ & $x$ & $x$ & $x$ & $x$ & & $x$ \\
\hline $\begin{array}{l}\text { Assessment of treatment emergent adverse } \\
\text { events }\end{array}$ & & $\bullet$ & & & & & $\rightarrow$ \\
\hline MRI & & $x$ & & & & & \\
\hline Sleep EEG & & $x$ & $x$ & $x$ & $x$ & & $x$ \\
\hline NPA & & $x$ & & & $x$ & & $x$ \\
\hline Brief assessment of cognitive performance & & $x$ & & $x$ & & & \\
\hline Cytokine profiles and auto-antibodies & & $x$ & & & & $x$ & \\
\hline Clobazam levels (2 times ${ }^{* * *}$ & & & $x$ & & $x$ & & \\
\hline
\end{tabular}

$*-t_{1}=$ Informative visit (both oral and written information), $t_{0}=$ Allocation and collection of baseline data. At least one week after $-t_{1}$ and if informed consent has been given, $\mathbf{t}_{1}=1$ month visit, $\mathbf{t}_{3}=3$ months visit, $\mathbf{t}_{\mathbf{6}}=6$ months visit, $\mathbf{t}_{8}=8$ months visit, $\mathbf{t}_{18}=18$ months visit

** treatment with clobazam OR steroids according to randomization

*** clobazam levels between $\mathrm{t} 1$ and $\mathrm{t} 3$ and between $\mathrm{t} 3$ and $\mathrm{t} 6$, in patients randomized to clobazam

MRI = Magnetic Resonance Imiging, EEG = Electroencephalography, NPA = Neuropsychological Assessment 
Chapter 8. Corticosteroids vs. clobazam for ESES (RESCUE ESES)

Although the stratified randomisation procedure intends to create two groups with equal patient characteristics, it is known that in randomised trials of $<1000$ patients, there still is a risk of bias by chance. ${ }^{25}$ Adjusted analyses are often performed to reduce the influence of the possible differences between the two treatment arms. ${ }^{26}$ Therefore, possible predictors of treatment outcome (i.e. known prognostic factors) will be included in a multivariate logistic / linear regression model. These prognostic factors are: age at ESES recording, time interval between ESES recording and inclusion, IQ levels and cognitive sum scores at enrolment, number of drugs administered before enrolment, aetiology (unknown, structural, metabolic, genetic, immune, infectious). We will also include cytokine and autoantibody profiles as possible predictors.

\section{Sample size calculation}

A formal sample size calculation is hampered by the fact that no previous trials with these interventions have been performed. Our recently performed meta-analysis of published cases with epileptic encephalopathy with ESES provides the basis for estimating the difference in proportions of successfully treated patients that might be expected between corticosteroids and benzodiazepines. In our meta-analysis, treatment success was defined as improvement in EEG (at least 25\% decrease in SWI) or cognition (10 IQ points or improvement defined by author). Difference in proportions were $25-30 \%$ between the two treatment groups in favour of corticosteroids. However, these results are of limited value because of the small sample size in the included studies and their mostly retrospective design.

In the RESCUE ESES study we aim to include a total of 130 children, of whom 65 will be randomized to treatment with corticosteroids and 65 to clobazam. This sample size permits to detect a difference of $25 \%$ in the proportion of successfully treated patients between the two treatment arms (for example 50\% vs 25\%). In fact, 116 patients are needed to identify this difference with a power of $80 \%$ and a two-sided alpha of $5 \%$, and we additionally account for a possible dropout rate of $10 \%$.

As mentioned above, success is defined as improvement of 0.75 of the standard deviation of either IQ or the cognitive sumscore. Differences of this magnitude have been reported in earlier observational (non-randomized) studies in this area. ${ }^{27}$ Displaying the primary outcome as a dichotomous value (instead of a continuous value), will lead to a conservative estimation of the difference in effect. Therefore, our sample size calculation is likely to be an overestimation of the required number of patients for the continuous outcomes. 
For continuous outcomes we can detect standardized mean differences or Cohen's $d$ of around 0.5 (power $=80 \%$, two-sided alpha of 5\%). For Cohen's $d$ an effect size of 0.2 to 0.3 is considered a "small" effect, around 0.5 a "medium" effect and above 0.8, a "large" effect. 28 This means that "medium" effects on secondary outcomes can be detected by our trial including 130 children. Furthermore, as the primary outcome can also be displayed as a continuous variable (IQ or cognitive sumscore), this also applies to analysis of the primary outcome as a continuous variable.

\section{Recruitment and promotion of participant retention}

Because encephalopathy with ESES is relatively rare, we initiated a collaboration with European centers with a high level of expertise in epilepsy. The trial was announced at several international congresses, in newsletters of national and international paediatric neurology and epilepsy associations as well as in patient magazines. Online as well as local training meetings for study personnel were organized. The study team is available for any questions or concerns from patients and their parents and an independent physician is available for questions or complaints. If patients discontinue participation in the study or a protocol deviation occurs, the baseline and outcome measures that have been collected before discontinuation will still be analysed, e.g. if discontinuation occurred after 6 months of follow up, the collected data will be included in primary outcome assessment. If they switch to the other treatment arm, but agree to continued follow-up according to the study protocol, their outcomes will still be analysed in the intention to treat analysis.

\section{Safety and tolerability}

In accordance with legal requirements, the investigator will inform study participants and the reviewing accredited Ethics Committees (EC) if any data or findings emerge during the conduct of the study that suggest that risks involved in participation may outweigh potential benefits.

Adverse events will be reported according to good clinical practice (GCP) guidelines, as specified in the study protocol.

\section{Monitoring}

Because both treatments in this trial are also given as part of standard patient care, the study was classified as a (pragmatic) low risk trial. A data safety and monitoring board (DSMB) was therefore not required. An initiation visit, annual monitoring visits and a closeout visit are performed in all centres in accordance with GCP guidelines. 
Chapter 8. Corticosteroids vs. clobazam for ESES (RESCUE ESES)

\section{Discussion}

Encephalopathy with ESES is characterized by sleep-induced epileptic activity accompanied by acquired cognitive deficits. Treatment aims at improving cognitive functioning. Current evidence regarding treatment efficacy is limited to mostly retrospective case series and indicates that conventional anti-epileptic drugs are often not effective, and that benzodiazepines and corticosteroids can provide greater benefit in improving cognition and EEG abnormalities. In patients with an operable structural lesion, surgery seems to be the most effective treatment.

The current study aims at comparing the efficacy of corticosteroids versus clobazam in improving cognitive functioning in children with ESES. The co-primary outcomes are cognitive functioning, as measured with total IQ scores and cognitive sumscores after 6 months. Secondary outcomes include cognitive functioning after 18 months, the spikewave index after 6 and 18 months, safety and tolerability, subjective assessment of daily functioning and whether disease severity and treatment effect can be predicted, among other characteristics, by measuring serum levels of cytokines or auto-antibodies.

The conduct of this multinational multicentre study in the rare population of patients with ESES is challenging for several reasons. Firstly, the differences in legislation and regulations between the involved countries and the requirement of obtaining ethics committee and competent authority approval for each of these countries, is difficult and time-consuming. Secondly, although clobazam and corticosteroids are widely prescribed in regular clinical care, in some countries labelling of the study drugs for this specific indication was considered to be required and posed a logistical challenge. Thirdly, each centre had their own requirements in terms of clinical trial contracts and this resulted in numerous email and telephone discussions between legal representatives. Altogether, these challenges caused considerable delay of the initiation of study at participating centres and resulted in withdrawal of several centres.

Despite the good intentions of local investigators, announcements on congresses, in newsletters, on websites and RESCUE ESES newsletters and promotional material, recruitment of patients in the centres where the study was initiated has so far been slower than expected. No general reasons have become apparent, but a few possible explanations have been proposed. Some patients did not participate because they considered the study investigations time consuming and were concerned that their treatment might be delayed. Others had a strong preference for one of the treatment arms, e.g. because of the ease of use of clobazam or presumed higher effectivity of corticosteroids. Furthermore, the investigators involved are only supported in trial management and not financially 
compensated for time spent on study procedures. In addition, some local investigators mentioned that it was difficult to influence local referral patterns and that they failed to promote referral of additional ESES patients for enrolment in the study.

To conclude, we believe that this study addresses an important open question and that the results may guide clinicians in choosing the best treatment for patients with epileptic encephalopathy with ESES.

\section{Trial status}

The study protocol (latest version: 11, 25 ${ }^{\text {th }}$ of November 2014) was approved by ethics committees for conduct in the University Medical Center Utrecht in the Netherlands, University Hospital (UZ) Brussel and University Hospital (UZ) Leuven in Belgium, University Hospital Freiburg, Epilepsy Center Kork (Kehl), the Northern German Epilepsy Center Raisdorf and Schön Klinik Vogtareuth in Germany, Filadelfia Epilepsy Hospital (Dianalund) in Denmark, Helsinki University Hospital in Finland, University Hospital Lyon (HCL), University Hospital Paris and University Hospital Strasbourg in France, University Hospital San Carlos (Madrid) in Spain, Great Ormond Street Hospital for Children (London), Royal Hospital for Sick Children Edinburgh and Royal Hospital for Sick Children Glasgow in the United Kingdom. Approval by the ethics committee of the University Children's Hospital Zurich, Switzerland is expected soon. The first patient was included on the 22 July 2014 and to date (2 April 2020) 43 patients have been included. The study end date is currently scheduled at 31 December 2020, but study extension will be considered.

\section{Declarations}

\section{Ethics approval and consent to participate}

Approval for the conduct of the study was received by the ethics committee of the University Medical Center Utrecht in December 2013 (protocol number 13-275 / NL43510.041.13) and subsequently by the ethics committees / institutional review boards of 15 other centres as specified above under "Trial status". Changes in the study protocol have been / will be proposed to ethics committees / institutional review boards, communicated to trial site principal investigators by email and in regular investigator meetings (online meetings and meetings at international congresses). Depending on the implications of the changes these have been / will also be communicated to patients / parents and the trial registry (ISRCTN). Since the first approval of the study protocol, two amendments have been submitted (approved in January 2016 and February 2018). These amendments had no impact on the patients that were already included in the study. Patients were included in the study if informed consent was obtained by their parents / legal representatives as described in the methods section (subheading "Informed consent"). The trial is conducted in accordance with the principles of the Declaration of 
Chapter 8. Corticosteroids vs. clobazam for ESES (RESCUE ESES)

Helsinki, as amended by the World Medical Association General Assembly in October 2013, with the ICH guidelines for Good Clinical Practice (CPMP/ICH/135/95, July 1996) and with all international laws and regulations (including EU Clinical Trial Directive 2001/20/EC) and national laws and regulations of the countries in which the clinical trial is performed. The protocol (version 11, 25 $5^{\text {th }}$ of November 2014) has been prepared and reported in accordance with the Standard Protocol Items: Recommendations for Interventional Trials (SPIRIT) checklist (appendix 1).

\section{Consent for publication}

Not applicable.

\section{Availability of data and materials}

Not applicable.

\section{Competing interests}

The authors declare that they have no competing interests.

\section{Funding and support}

This study is funded by the Dutch Epilepsy Fund (Epilepsiefonds, grant 13-17) and the Wilhelmina Children's Hospital Research Fund (grant R2634). The funding sources had no direct involvement in the conduct of the study.

The European Clinical Research Infrastructrure Network (ECRIN) provides support for the European extension of the study via their network of clinical trial units. Their services include translation of study documents, support for ethics committee and competent authority submission and monitoring of study sites.

The funding sources had no involvement in the design of the study and will have no direct involvement in the analysis and interpretation of findings, writing of the manuscript and the decision to submit the report for publication. ECRIN provides logistical support and monitoring during the conduct of the study, but will not be directly involved in data collection.

\section{Authors' contributions}

FEJ designed the trial in collaboration with BVdM, AA, EP, FSSL, HCVT and KPJB. FEJ applied for funding, established collaborations and is the principal investigator of the trial. BvdM does the coordination of the study and patient recruitment in collaboration with FEJ. BvdM wrote the first version of this manuscript. All authors together constitute the steering committee of the trial and are involved in decision making regarding design, 
conduct and dissemination of the study. All authors read and revised the manuscript and approved the submission of the study protocol.

\section{Acknowledgements}

The study is conducted by the RESCUE ESES study group which constitutes of current and previous collaborators, and includes Anna Jansen (Brussels, Belgium), Patrick van Bogaert (Brussels, Belgium), Lieven Lagae (Leuven, Belgium), Guido Rubboli (Dianalund, Denmark), Eija Gaily (Helsinki, Finland), Pierangelo Veggiotti (at start of study working in Pavia, Italy, currently working in Milano, Italy), Gaetano Cantalupo (Verona, Italy), Giuseppe Gobbi (Bologna, Italy), Dana Craiu (Bucharest, Romania), Petia Dimova (Sofia, Bulgaria), Thomas Bast (Kehl, Germany), Julia Jacobs (Freiburg, Germany), Sarah von Spiczak (Schwentinental-Raisdorf, Germany), Anja Lübbig (Vogtareuth, Germany), Stéphane Auvin (Paris, France), Anne de Saint-Martin (Strasbourg, France), J. Helen Cross (London, United Kingdom), Richard Chin (Edinburgh, United Kingdom), Sameer Zuberi (Glasgow, United Kingdom), Irene Garcia Morales (Madrid, Spain), Georgia Ramantani (Zurich, Switzerland) and all authors of this manuscript.

We thank all study participants and their parents / caregivers for their participation in the trial and their patience during the conduct of the study.

We thank all co-investigators, collaborating paediatric neurologists, neuropsychologists, clinical neurophysiologists, technicians, nurses, outpatient assistants, laboratory employees and anyone else involved in the conduct of the study for their efforts and support.

We thank dr. Guido Rubboli for his suggestions regarding the protocol for the registration of EEGs in the study.

We thank Carolijn Kesler for her continued support in the legislatory and contractual challenges that we faced during the preparations and conduct of the study.

We thank Eltje Bloemen for her general support and enthusiasm with the conduct of the trial, knowledge about good clinical practice guidelines, approachability for ESES patients and help with the international coordination of the study.

We thank Judith Meester for her support with the conduct of the trial. 
Chapter 8. Corticosteroids vs. clobazam for ESES (RESCUE ESES)

\section{References}

1. Patry G, Lyagoubi S, Tassinari CA. Subclinical "electrical status epilepticus"induced by sleep in children. Arch Neurol. 1971;24(3):242-252. doi:http://dx.doi.org/10.1001/archneur.1971.00480330070006

2. Landau WM, Kleffner FR. Syndrome of acquired aphasia with convulsive disorder in children. Neurology. 1957;7(8):1241, 8 pages following 1241. doi:10.1212/WNL.7.8.523

3. Fernández IS, Chapman KE, Peters JM, et al. The tower of Babel: Survey on concepts and terminology in electrical status epilepticus in sleep and continuous spikes and waves during sleep in North America. Epilepsia. 2013;54(4):741-750. doi:10.1111/epi.12039

4. Nickels K, Wirrell E. Electrical Status Epilepticus in Sleep. Semin Pediatr Neurol. 2008;15(2):50-60. doi:10.1016/j.spen.2008.03.002

5. Sánchez Fernández I, Loddenkemper T, Peters JM, Kothare S V. Electrical status epilepticus in sleep: Clinical presentation and pathophysiology. Pediatr Neurol. 2012;47(6):390-410. doi:10.1016/j. pediatrneurol.2012.06.016

6. Hughes JR. A review of the relationships between Landau-Kleffner syndrome, electrical status epilepticus during sleep, and continuous spike-waves during sleep. Epilepsy Behav. 2011;20(2):247253. doi:10.1016/j.yebeh.2010.10.015

7. Ballaban-Gil K, Tuchman R. Epilepsy and epileptiform EEG: Association with autism and language disorders. Ment Retard Dev Disabil Res Rev. 2000;6(4):300-308. doi:10.1002/10982779(2000)6:4<300::AID-MRDD9>3.0.CO;2-R

8. Tassinari CA, Rubboli G. Encephalopathy related to Status Epilepticus during slow Sleep: current concepts and future directions. Epileptic Disord. 2019. doi:10.1684/epd.2019.1062

9. Bölsterli BK, Schmitt B, Bast T, et al. Impaired slow wave sleep downscaling in encephalopathy with status epilepticus during sleep (ESES). Clin Neurophysiol. 2011;122(9):1779-1787. doi:10.1016/j. clinph.2011.01.053

10. Kersbergen KJ, De Vries LS, Leijten FSS, et al. Neonatal thalamic hemorrhage is strongly associated with electrical status epilepticus in slow wave sleep. Epilepsia. 2013;54(4):733-740. doi:10.1111/ epi.12131

11. van den Munckhof B, Zwart AF, Weeke LC, et al. Perinatal thalamic injury: MRI predictors of electrical status epilepticus in sleep and long-term neurodevelopment. Neurolmage Clin. 2020. doi:10.1016/j. nicl.2020.102227

12. Lemke JR, Lal D, Reinthaler EM, et al. Mutations in GRIN2A cause idiopathic focal epilepsy with rolandic spikes. Nat Genet. 2013;45(9):1067-1072. doi:10.1038/ng.2728

13. Lesca G, Rudolf G, Bruneau N, et al. GRIN2A mutations in acquired epileptic aphasia and related childhood focal epilepsies and encephalopathies with speech and language dysfunction. Nat Genet. 2013;45(9):1061-1066. doi:10.1038/ng.2726

14. de Vries EE, van den Munckhof B, Braun KPJ, van Royen-Kerkhof A, de Jager W, Jansen FE. Inflammatory mediators in human epilepsy: A systematic review and meta-analysis. Neurosci Biobehav Rev. 2016;63:177-190. doi:10.1016/j.neubiorev.2016.02.007

15. Connolly AM, Chez MG, Pestronk A, Arnold ST, Mehta S, Deul RK. Serum autoantibodies to brain in Landau-Kleffner variant, autism, and other neurologic disorders. J Pediatr. 1999;134(5):607-613. doi:10.1016/S0022-3476(99)70248-9

16. Van Den Munckhof B, De Vries EE, Braun KPJ, et al. Serum inflammatory mediators correlate with disease activity in electrical status epilepticus in sleep (ESES) syndrome. Epilepsia. 2016;57(2):e45-e50. doi:10.1111/epi.13274

17. Giovanardi Rossi P, Parmeggiani A, Posar A, Scaduto MC, Chiodo S, Vatti G. Landau-Kleffner syndrome (LKS): long-term follow-up and links with electrical status epilepticus during sleep (ESES). Brain Dev. 1999;21(2):90-98. doi:10.1016/\$0387-7604(98)00071-0 
18. Pera MC, Brazzo D, Altieri N, Balottin U, Veggiotti P. Long-term evolution of neuropsychological competences in encephalopathy with status epilepticus during sleep: A variable prognosis. Epilepsia. 2013;54(SUPPL.7):77-85. doi:10.1111/epi.12313

19. van den Munckhof B, van Dee $V$, Sagi $L$, et al. Treatment of electrical status epilepticus in sleep: A pooled analysis of 575 cases. Epilepsia. 2015;56(11):1738-1746. doi:10.1111/epi.13128

20. van den Munckhof B, Alderweireld C, Davelaar S, et al. Treatment of electrical status epilepticus in sleep: Clinical and EEG characteristics and response to 147 treatments in 47 patients. Eur J Paediatr Neurol. 2018;22(1):64-71. doi:10.1016/j.ejpn.2017.08.006

21. Liukkonen E, Kantola-Sorsa E, Paetau R, Gaily E, Peltola M, Granström ML. Long-term outcome of 32 children with encephalopathy with status epilepticus during sleep, or ESES syndrome. Epilepsia. 2010. doi:10.1111/j.1528-1167.2010.02578

22. Sánchez Fernández I, Chapman K, Peters JM, et al. Treatment for continuous spikes and waves during sleep (CSWS): Survey on treatment choices in North America. Epilepsia. 2014;55:1099-1108. doi:10.1111/epi.12678

23. Veggiotti P, Pera MC, Teutonico F, Brazzo D, Balottin U, Tassinari CA. Therapy of encephalopathy with status epilepticus during sleep (ESES/CSWS syndrome): An update. Epileptic Disord. 2012;14(1):1-11. doi:10.1684/epd.2012.0482

24. van Coevorden-Hameete MH, Titulaer MJ, Schreurs MWJ, de Graaff E, Sillevis Smitt PAE, Hoogenraad CC. Detection and Characterization of Autoantibodies to Neuronal Cell-Surface Antigens in the Central Nervous System. Front Mol Neurosci. 2016. doi:10.3389/fnmol.2016.00037

25. Nguyen TL, Collins GS, Lamy A, et al. Simple randomization did not protect against bias in smaller trials. J Clin Epidemiol. 2017. doi:10.1016/j.jclinepi.2017.02.010

26. Saquib N, Saquib J, loannidis JPA. Practices and impact of primary outcome adjustment in randomized controlled trials: Meta-epidemiologic study. BMJ. 2013. doi:10.1136/bmj.f4313

27. Kramer U, Sagi L, Goldberg-Stern H, Zelnik N, Nissenkorn A, Ben-Zeev B. Clinical spectrum and medical treatment of children with electrical status epilepticus in sleep (ESES). Epilepsia. 2009;50(6):15171524. doi:10.1111/j.1528-1167.2008.01891

28. Cohen J. Statistical Power Analysis for the Behavioural Science (2nd Edition). In: Statistical Power Anaylsis for the Behavioural Science (2nd Edition). ; 1988:25-27 

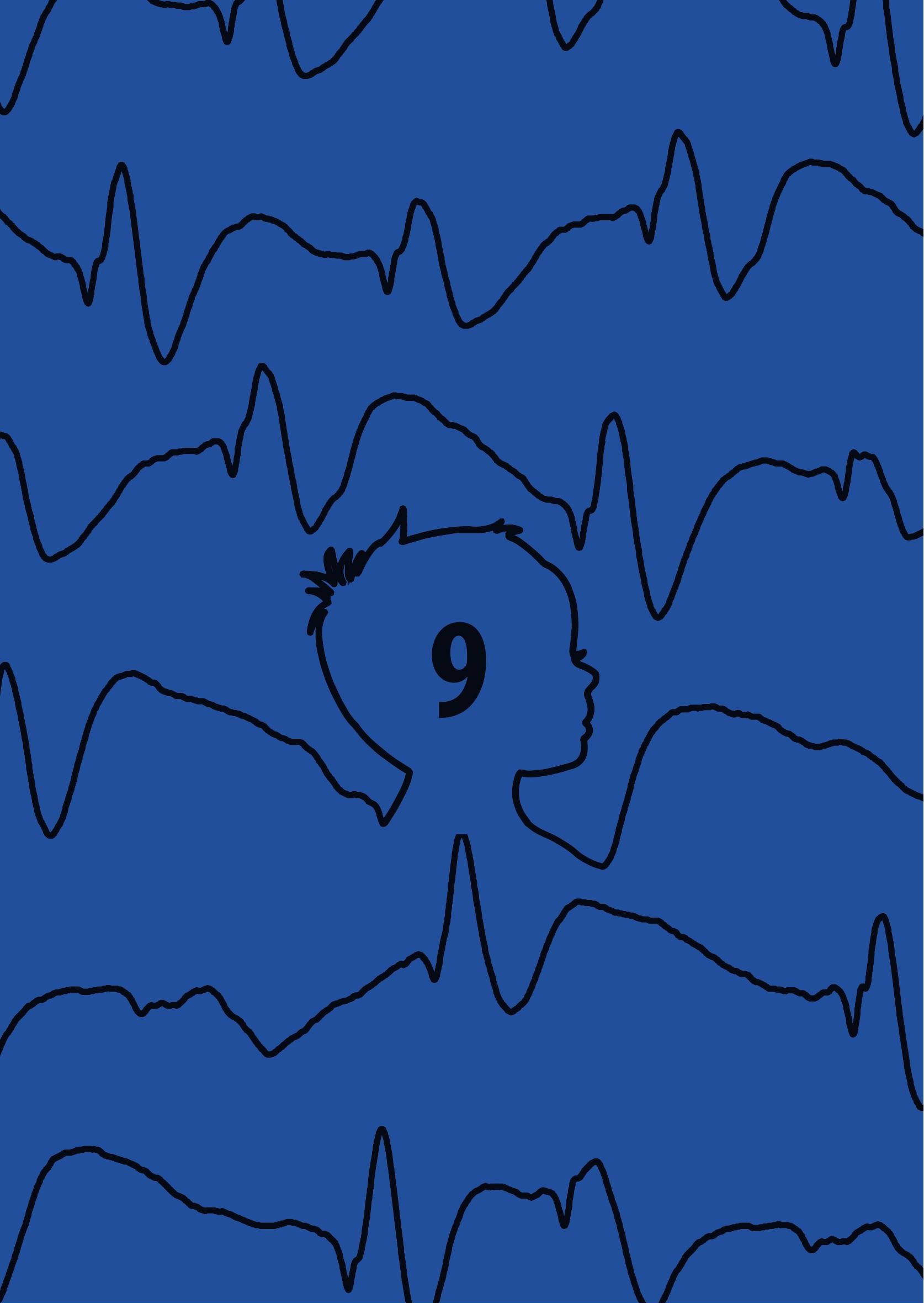


\section{Chapter 9}

\section{Summary}


The aim of the studies described in this thesis was to 1) to improve early recognition of children at risk of ESES development, 2) to increase our knowledge of ESES aetiology and pathophysiological mechanisms, and 3) to provide more evidence on optimal treatment of children with encephalopathy with ESES. We studied several aspects of this epileptic encephalopathy, which are summarised and discussed below.

\section{PART I. Aetiology and Pathophysiology}

In chapter 2, we describe that nearly all patients with perinatal thalamic injury developed ESES spectrum abnormalities during follow-up. Residual thalamic volume on an MRI performed after 3 months is predictive of long-term neurodevelopment: the patients with the largest thalamic volume had the highest total IQ scores during follow-up. We also explored whether alteration of structural connectivity may provide an explanation for cognitive deficits in children who suffered from perinatal thalamic injury. Higher fractional anisotropy and lower mean diffusivity at the MRI performed later during childhood were found to be correlated to total IQ. This suggests that children with relatively welldeveloped functional integrity of the thalamus benefit from preserved thalamic control over their developing brain.

In chapter 3, we present the results of a systematic review and meta-analysis of studies that investigated biomarkers of inflammation in the broader context of human epilepsy. We found that in different epilepsy aetiologies, and within different media (serum, cerebrospinal fluid [CSF], brain tissue), different patterns of activated inflammatory proteins were reported. The widely studied cytokines, such as IL-6, IL-1 ra, and IL-1 $\beta$, were found to be raised in several studies across all media, while the chemokines were raised specifically in brain tissue. In the aggregate meta-analysis, we found that in serum the proinflammatory cytokines IL-6 and IL-17 were higher in epilepsy patients than in controls, while in CSF the pro-inflammatory IL-1 $\beta$ and anti-inflammatory IL-10 were raised. From chapter 3, we conclude that in patients with epilepsy several inflammatory pathways are activated. The difference in inflammatory profiles between serum, CSF and brain tissue suggests that local brain inflammation as well as a more general inflammatory response play a role. Whether these inflammatory pathways are part of a cascade that contributes to the development of epilepsy, or are merely an epiphenomenon of seizures, is unclear. Future studies may further clarify their role and explore the potential of targeted antiinflammatory treatment strategies. 
In chapter 4, we explored whether levels of inflammatory mediators are higher in serum of patients with ESES as compared to controls. We found that, among the 16 proteins investigated, levels of 7 proteins were significantly different in patients ( 5 higher, 2 lower) from those in controls. IL-6 levels decreased significantly after immunomodulatory therapy and this was accompanied by improvement of the EEG-abnormalities and neuropsychological test results. We hypothesize that activity of several cytokines correlates with disease activity and speculate that (at least in some patients with ESES), modification of this inflammatory activation may be beneficial.

In chapter 5, we studied if neurodevelopmental deficits and behavioural symptoms in children with ESES can be explained by disturbed sleep physiology. The synaptic homeostasis hypothesis describes the process of the creation of new synapses and strengthening of existing synapses during wakefulness (synaptic potentiation), balanced by synaptic downscaling during sleep. Synaptic downscaling is the process of selective reduction of the number and strength of synapses, thereby preserving the most important connections. Previous studies have shown that slow wave activity (an important EEG characteristic of non-REM sleep), especially slow wave slope, is an indirect marker of synaptic density. In healthy controls, an overnight reduction in slow wave slope reflects the process of synaptic downscaling. In patients with encephalopathy with ESES, a reduced overnight slow wave slope decline was reported. We tested the hypothesis that epileptic activity in non-REM sleep in children with encephalopathy with ESES, interferes with synaptic downscaling and thereby influences cognitive functioning and behaviour. We found that, in children with ESES, the overnight reduction in slow wave slope is more impaired in the epileptic focus electrodes, as compared to the non-focus electrodes. This suggests that slow wave activity is locally disturbed by epileptic activity. We also found that patients with behavioural problems, as compared to those without, show a more hampered slow wave slope decline (i.e. on average a less pronounced overnight slow wave slope decline). We did not find a correlation between the overnight slow wave slope course and neurodevelopmental test results. Whether this reflects insufficient study power, insensitivity of neurodevelopmental tests to probe cognitive fluctuations during the course of ESES, or a truly absent link between slow wave slope course and neurodevelopmental function, remains to be elucidated.

\section{PART II. Treatment}

In chapter 6, we present a systematic review of the literature on the treatment of encephalopathy with ESES. We also present a pooled analysis of 575 published cases, to assess to what extent cognitive or EEG improvement relate to treatment with 
conventional anti-epileptic drugs, benzodiazepines, corticosteroids and surgery. Twentynine authors contributed additional information to increase the completeness of the dataset of published cases. To reduce the influence of publication bias, we also performed a subgroup analysis of consecutive cohort patients. We found that any improvement either in cognition or EEG - was reported in $49 \%$ of patients treated with conventional anti-epileptic drugs, $68 \%$ with benzodiazepines, $81 \%$ with corticosteroids and $90 \%$ with surgery. In the subgroup of consecutively included patients, a markedly lower proportion showed any improvement with AED and benzodiazepines, while the high improvement percentage for corticosteroids and surgery was relatively preserved. Improvement after treatment was not only related to treatment type. Children with abnormal development before ESES onset were less likely to improve after treatment. Due to the mostly retrospective nature and small cohort size of most included studies, results have to be interpreted with caution. Nevertheless, the findings suggest that treatment with corticosteroids and surgery are relatively effective.

In chapter 7, we analysed if EEG improvement (SWI decrease) after treatment is associated with cognitive improvement in children with encephalopathy with ESES. In this retrospective single centre cohort study of 147 treatments in 47 patients, we found that SWI decrease is associated with subjective cognitive improvement, while we did not find a significant association between EEG improvement and IQ change. We also showed that steroid treatment (as compared to non-steroid treatment) was related to cognitive improvement at first follow-up, while higher age at diagnosis was related to improvement at last follow-up of treatment. Although these treatment data from a retrospective cohort have to be interpreted carefully, the results may help clinicians in their choice of treatment and interpretation of findings after initiation of treatment in a child with encephalopathy with ESES.

Chapter 8 describes the design of a European Randomised controlled trial (acronym RESCUE ESES) comparing corticosteroids and clobazam treatments in children with encephalopathy with ESES. The aim of treatment is to improve cognitive functioning. The trial requires recruitment of 130 patients, 2 to 12 years of age, to be treated according to randomisation for 6 months. Primary outcome is cognitive functioning at 6 months and secondary outcomes include cognitive functioning after 18 months, EEG findings (SWI), side effects and seizure frequency. We will also investigate whether inflammatory markers (cytokines and auto-antibodies) are associated with treatment effect. The first patient was included in July 2014. 


\section{Key points box}

- Encephalopathy with ESES is a childhood epilepsy syndrome characterised by a cognitive or behavioural deterioration and sleep-induced epileptiform activity.

\section{Part I. Aetiology and Pathophysiology}

- Children with perinatal thalamic injury are at high risk of developing ESES and higher residual thalamic volume predicts better neurodevelopmental outcome.

- Several inflammatory mediators are highly activated in people with epilepsy.

- In children with ESES, serum levels of several inflammatory mediators are higher than in controls and reduction in IL-6 with treatment is accompanied by clinical improvement.

- Overnight sleep slow wave homeostasis, reflecting synaptic homeostasis, is impaired in children with ESES and this impairment is most severe in children with behavioural problems.

\section{Part II. Treatment}

- In children with ESES, benzodiazepines and corticosteroids are more effective than conventional anti-epileptic drugs in improving cognition or EEG. In cases with a clear unilateral structural lesion, surgery is the most effective treatment.

- Reduction in epileptiform activity (SWI) after treatment is associated with subjective improvement in cognitive functioning, but not reflected by significant total IQ change.

- To assess the best medical treatment for ESES, a randomised controlled trial comparing clobazam and corticosteroid treatment (RESCUE ESES) is currently conducted. Outcome measures include neuropsychological test results, EEG findings and side-effects. Possible predictors (including inflammatory mediators) are analysed. 

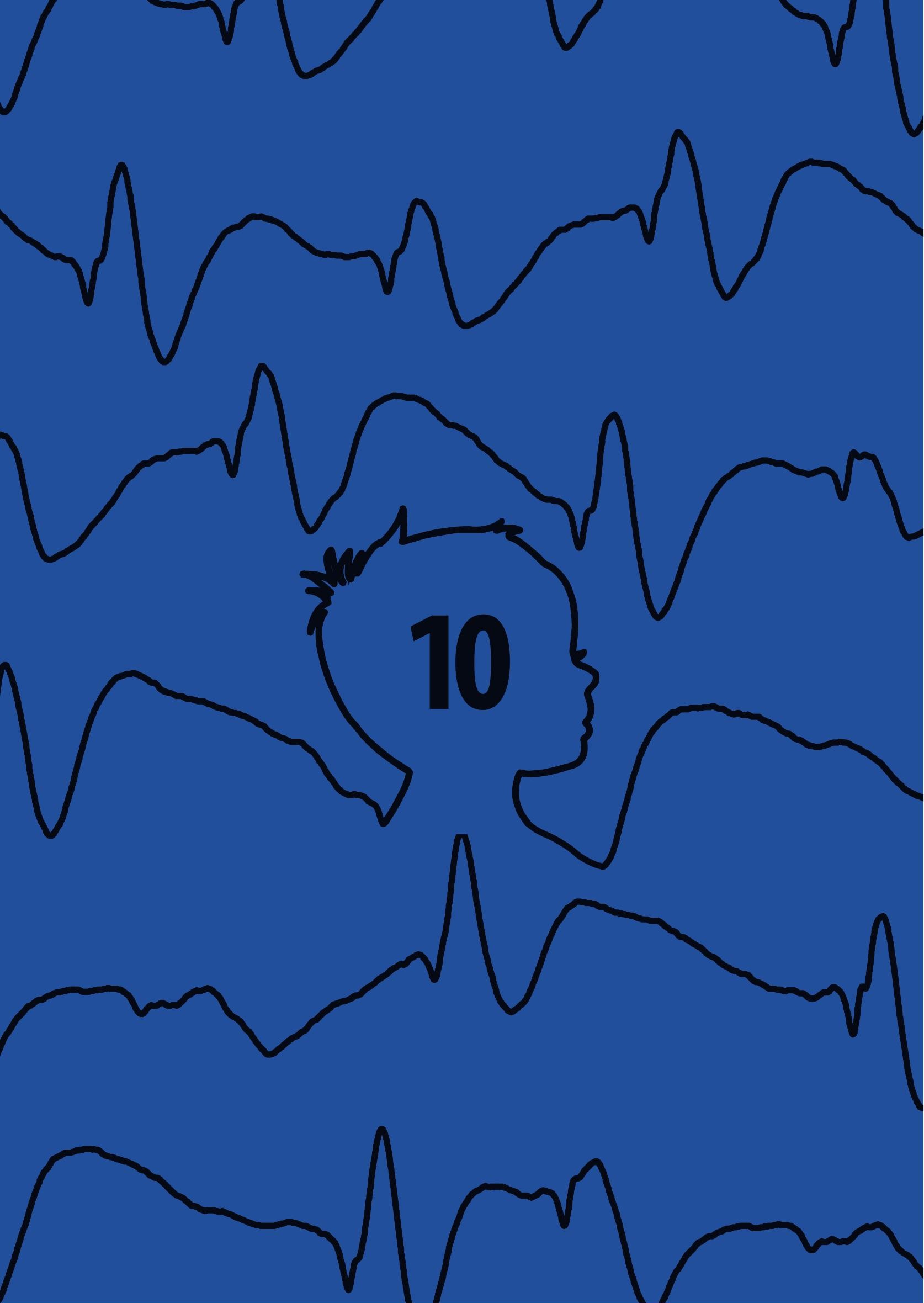


\section{Chapter 10}

General discussion 


\section{When to consider encephalopathy with ESES?}

The clinical manifestations associated with ESES EEG abnormalities are highly variable, ranging from normal development and behaviour to acquired aphasia or global developmental regression., ${ }^{1,2}$ In a child of at least 2 years of age with new-onset learning problems or behavioural regression, a sleep EEG should be considered. In most cases, an epileptic seizure precedes or accompanies the (worsening of) neurodevelopmental deficits, but cases without seizures exist. Especially in the case of an acquired auditory agnosia or aphasia, seizures may be absent and a high index of suspicion is required., ${ }^{3,4}$ However, routine performance of sleep EEGs in children with autism or ADHD is not recommended, as they frequently have epileptiform activity in sleep and there is no evidence for a causative relation with their symptoms. ${ }^{5}$

\section{Definition of encephalopathy with ESES}

Over the years, different criteria for the diagnosis of (encephalopathy with) ESES have been used. Patry et al. first described ESES and stated that there had to be continuous epileptiform activity in sleep, defined as occupying at least $85 \%$ of the slow wave tracing. This pattern had to be present in at least three sleep recordings over a period of at least one month. They also noted that all six children in their study had some kind of "mental retardation".6 Since this first report, several different criteria for epileptiform activity and cognitive consequences have been suggested to define this epilepsy syndrome ${ }^{7-10}$ Although no consensus exists, in our opinion, two core criteria are essential to the diagnosis of encephalopathy with ESES in children:

A) Severe epileptiform activity induced by non-REM sleep; this implies that a substantial proportion of sleep is affected by epileptiform activity and that there is a clear relation to non-REM sleep (i.e. it is not, or to a much lesser extent, present during wakefulness).

B) A neurodevelopmental deterioration that is likely related to the sleep-induced epileptiform activity.

Criterion A (EEG) can be further specified according to the quantity of epileptiform activity (e.g. the spike-wave index describing the proportion of time affected by epileptiform activity) and the localisation of epileptiform activity (e.g. focal vs. generalised, unilateral vs. bilateral). We suggest that a minimum spike-wave index (SWI) of 50\% is probably required to cause clinical symptoms, which define encephalopathy with ESES, and that bilateral epileptiform activity is, in general, more severe than unilateral epileptiform activity. Aggravation of epileptiform activity in sleep is frequently seen in patients with benign focal epilepsies of childhood and in children with ADHD and autism and it is unclear whether this has any influence on their functioning. In most of these cases, the SWI does not exceed $50 \% .^{5,11}$ 
Criterion B (neurodevelopment) is ideally based on repeated neuropsychological assessment showing a deterioration. However, in previously healthy children a baseline measurement (before the clinical symptoms emerge) is generally not available and in these children the history taken from parents and school reports is the best information available. The severity of the developmental deficit can be specified as a delay (still ongoing development, but slower than expected), arrest (not progressing anymore) or regression (losing skills).

Considering the large heterogeneity in EEG findings and clinical symptoms, encephalopathy with ESES, or ESES syndrome, represents a diverse spectrum. Every patient is different and the impression that epileptiform activity in sleep is impacting on development is the cornerstone of diagnosis and treatment decisions. For the purpose of research, the spectrum can be categorised as "typical ESES syndrome", representing the most severe end of the spectrum, or "atypical ESES syndrome" for cases with less severe EEG abnormalities or less deviation from their developmental trajectories. The inclusion criteria for our randomised controlled trial (RESCUE ESES) are based on this categorisation. Figure 1 illustrates the spectrum of ESES patients and the suggested categories.

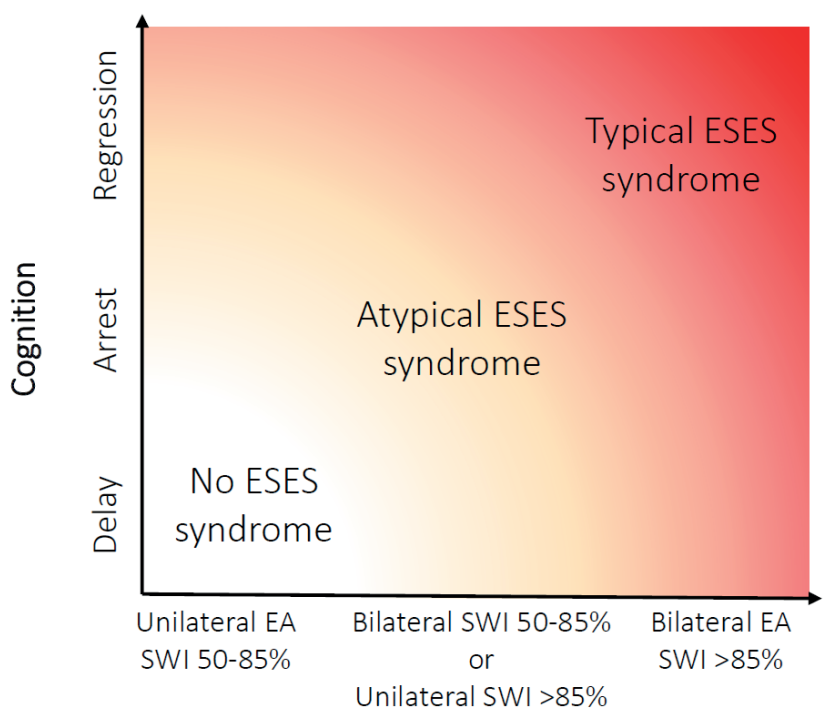

EEG

Figure 1: Spectrum of ESES syndrome 


\section{Thalamic injury}

In children with encephalopathy with ESES, diverse aetiologies have been reported, including perinatal or early thalamic injury or hypoplasia, polymicrogyria, genetic abnormalities (GRIN2A mutations), as well as cases of unknown aetiology.12-16 We added evidence that children with perinatal thalamic injury are at a very high risk of developing ESES.17 We also found a very strong correlation between residual thalamic volume and long-term total $I Q$, which persists in a multivariate model with total brain volume and SWI. This suggests that injury of the thalamus, in addition to playing a role in the development of ESES, has an impact on neurodevelopment. Furthermore, this raises the question whether, in the context of thalamic injury, the (severity of) epileptiform activity in sleep (SWI) influences neurodevelopment or whether it is just a marker of the severity of thalamic injury. This question is illustrated in figure 2.

\section{thalamic volume / structure loss}
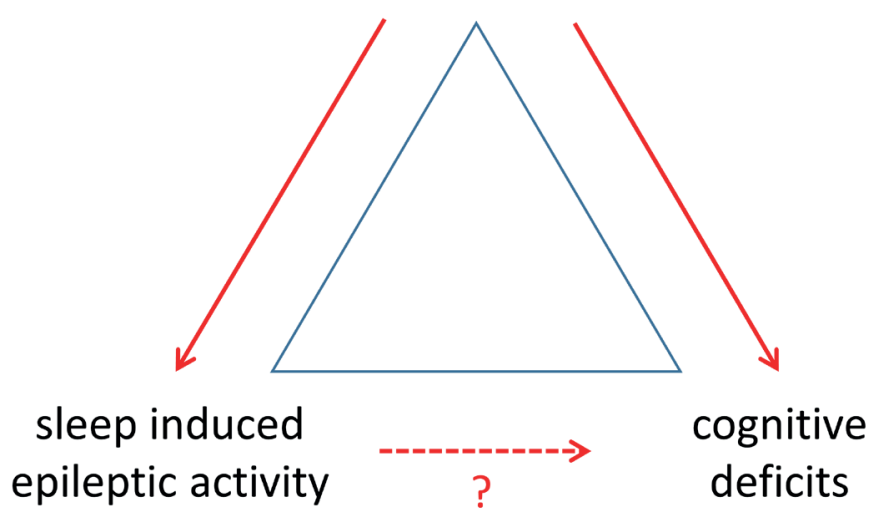

Figure 2: Thalamus, epileptiform activity and cognition

Previous studies, in a broader context of ESES patients with a range of aetiologies, suggested that the (severity of) epileptiform activity does relate to the cognitive functioning. ${ }^{18-20} \mathrm{We}$ found that improvement of cognitive functioning is related to reduction of epileptiform activity with treatment. ${ }^{21}$ Although it seems likely that the sleep related epileptiform activity in children with ESES after thalamic injury adversely affects cognition and behaviour, this remains to be proven. Larger studies in this population may show whether severity of epileptiform activity in sleep (SWI) has an impact on neurodevelopment, in addition to the impact of thalamic injury. 


\section{Inflammation}

Epilepsy is seen as a symptom of several inflammatory syndromes. In Rasmussen encephalitis, severe hemispheric inflammation leads to frequent refractory epileptic seizures and sometimes epilepsia partialis continua. The emergence of inflammation in this syndrome, and why it is generally confined to one hemisphere, is incompletely understood. An early role for T-cells and microglia is suggested. In many cases hemispherectomy is the only way to stop seizures and prevent further cognitive decline.22 Anti-NMDA receptor encephalitis is characterised by psychiatric manifestations, insomnia, dyskinesia and seizures. In patients with this disorder, antibodies to a subunit of the NMDA receptor are thought to be causative and immunomodulatory treatment is often effective in reducing severity and duration of symptoms, including seizures.23-25 These examples, along with other auto-immune and inflammatory conditions associated with epilepsy, suggest that inflammation may be a causative factor for epilepsy.26,27 In our meta-analysis of inflammatory mediators in epilepsy, we explored whether inflammatory activation is present in a broader context of epilepsy and found that levels of IL-6, IL-17 were different in serum of epilepsy patients as compared to controls. In cerebrospinal fluid, IL-1 $\beta$ and IL-10 were found to differ significantly from controls. Several of the included studies reported chemokines that were exclusively different in brain tissue.28 These findings suggest that both systemic and local inflammatory processes are activated in patients with epilepsy. A core question is whether inflammation is the cause or consequence of epilepsy, or both. Several studies have described the neuromodulatory properties of inflammatory proteins and their impact on neuronal excitability.29-33 This suggests that inflammatory proteins are part of a cascade that leads to epilepsy (i.e. epileptogenesis). Figure 3 is a schedule showing where in the cascade of epileptogenesis we suggest inflammation may contribute.

\section{Brain injury}

(stroke, infection, cortical malformation)

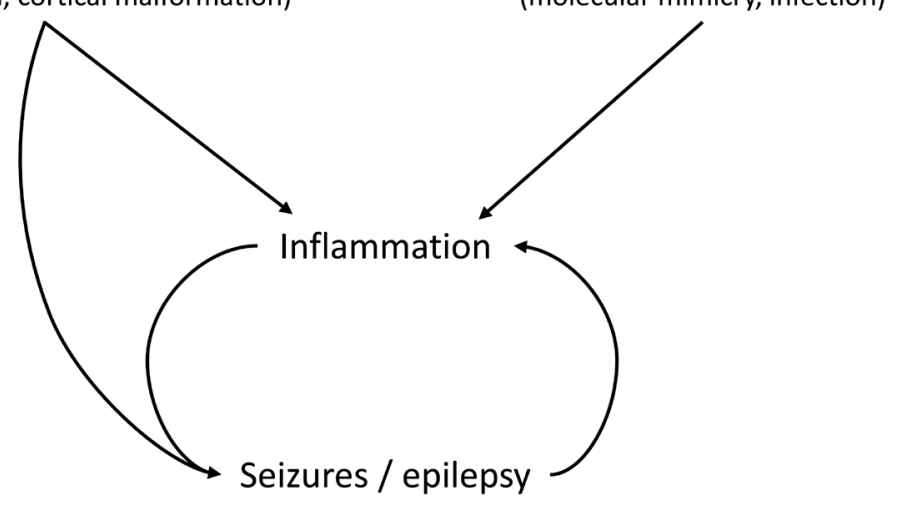

Figure 3: Simplified schedule of proposed role of inflammation in epileptogenesis 
Chapter 10. General discussion

In children with ESES, several studies reported a benefit of treatment with immunomodulatory agents, such as ACTH, corticosteroids and immunoglobulins.34-39 This suggests that inflammation may cause or sustain near-continuous epileptiform activity during sleep. A previous study reported increased activity of IL-6 in 4 children with epileptic encephalopathy with ESES. ${ }^{40}$ We compared serum cytokine levels between 11 patients with ESES and healthy controls and found that, in addition to IL-6, several other inflammatory proteins were significantly different in ESES patients. We are currently conducting a study comparing inflammatory proteins in brain tissue of operated ESES patients with brain tissue of epilepsy controls and non-epilepsy controls. Pilot data of fresh frozen brain tissue of 4 ESES patients, 4 epilepsy controls and 6 autopsy controls shows that expression of many inflammatory proteins is higher in epilepsy patients. IL-20 and CCL1 levels were higher in all epilepsy patients (ESES and epilepsy controls) than in any autopsy control. The activation of IL-20, which facilitates communication between leukocytes and endothelial cells, might be caused by the surgical procedures performed in the epilepsy patients. ${ }^{41}$ However, CCL1, involved in attraction of leukocytes and microglia, may be a sign of focal brain inflammation in the epileptic brain tissue. Three out of four ESES patients had higher CXCL7 levels than any epilepsy or autopsy control. This protein has pro-inflammatory and angiogenic properties and could be involved in focal cerebral inflammation in patients with ESES. The chemokines are an interesting group of proteins in the pathophysiology of epilepsy, as recent insights suggest that they can modulate neural excitability and some chemokines may even serve as neurotransmitters. ${ }^{42,43}$

If inflammation is an important factor in the process of epileptogenesis and may sustain seizure activity, this suggests opportunities for treatment. Corticosteroids have been widely studied in children with epileptic encephalopathies, such as West syndrome and ESES. ${ }^{39,44}$ By stimulating the glucocorticoid receptor, they have a broad range of actions and can be effective in many different diseases. However, the disadvantage of their broad therapeutic profile is a broad range of potential side-effects, which limits the possibilities for long-term treatment. ${ }^{45}$ Assessing the inflammatory profile in patients with epilepsy may help decide if immunomodulatory treatment is indicated and which option is preferred. Whilst recognising that this currently is not part of standard clinical practice, promising results have been reported from treatment with several drugs targeting specific aspects of the immune system. For example, VX765, which inhibits IL-1 $\beta$ synthesis, was shown to significantly reduce seizure frequency in patients with drug-resistant focal epilepsy as compared to placebo. ${ }^{46}$ Promising effects of drugs targeting several other specific antiinflammatory proteins and receptors in patients with epilepsy were reported, although mostly based on case series. ${ }^{33}$ Hopefully, in the near future, assessing the inflammatory profile of patients with epilepsy, especially children with ESES, will help clinicians find 
the pathophysiological mechanisms and choose a targeted treatment for each individual patient.

\section{Pathophysiology: Linking EEG abnormalities to cognitive and behavioural disturbances.}

The International League Against Epilepsy (ILAE) defined the concept of an epileptic encephalopathy as "where the epileptic activity itself contributes to severe cognitive and behavioural impairments above and beyond what might be expected from the underlying pathology alone".47,48 Epileptic encephalopathy with electrical status epilepticus in sleep (ESES) may serve as a classic example: children with an underlying structural or genetic abnormality that may already have some impact on their development, at some point show ESES on EEG recordings and an acquired developmental arrest or regression may occur. In children with ESES of unknown aetiology who previously had a normal development, the impact of ESES on their cognitive functioning is even more apparent. However, how and to what extent do the ESES EEG abnormalities contribute to cognitive deficits and behavioural symptoms? While it seems apparent that near-continuous epileptiform activity in sleep impairs the recuperative function of sleep, until recently, little was known about the mechanisms behind this relation.

The synaptic homeostasis hypothesis may be a valuable model to explain this presumed relationship. It describes the process called synaptic potentiation in which novel experiences during wakefulness lead to the formation of new synaptic connections and strengthening of some existing connections. During sleep, this is balanced by synaptic downscaling, i.e. a selective reduction in the number and strength of synapses. This selective reduction is thought to be essential to keep the brain network efficient and regain space for novel experiences during the subsequent day.49-52 It was found that the cortical firing rate and synchrony is relatively high after sustained wakefulness and relatively low after sustained sleep.53 In the EEG, slow waves are characteristic of nonREM sleep and represent synchronous oscillations of large number of cortical neurons. The process of synaptic downscaling is reflected by relatively large slow waves in early sleep, whilst the amount and size of slow waves decrease overnight.54-56 Previous studies found that, in children with ESES, overnight slow wave reduction, as a marker of synaptic downscaling, is hampered.57,58 They also reported partial normalisation of synaptic downscaling after resolution of ESES.59 In chapter 5, we added evidence that overnight slow wave reduction is impaired in children with ESES and that this impairment is more severe in the electrodes covering the areas with most pronounced sleep-induced epileptic activity. This suggests that, indeed, slow wave activity and synaptic downscaling are locally influenced by epileptic activity. How epileptic activity during sleep influences slow wave homeostasis and synaptic downscaling is incompletely understood. Some 
authors suggest a transformation of "physiological sleep slow waves" to "pathological sleep slow waves", i.e. spike-waves. If they occupy a substantial part of slow wave sleep, these spike-waves would have a detrimental effect on the overnight downscaling process. The scarce "physiological slow waves" that are still present, would thus not show normal overnight sleep slow wave decline. 58,60,61

Whether the severity of epileptiform activity and impairment of slow wave homeostasis is related to the severity of cognitive deficits was investigated by several studies. Galer et al. reported that overnight recall performance was worse in children with idiopathic focal epilepsy as compared to healthy controls. Interestingly, they also found that higher spike-wave index during sleep in these children was related to lower overnight recall performance. ${ }^{11}$ In another study, it was found that frequent spikes during sleep were related to reduced homeostatic decline in slow wave activity and this, in turn, was related to performance on a visual learning task. ${ }^{62}$ We assessed whether overnight slow wave slope decline in children with ESES was associated with neuropsychological assessment (NPA) test results and did not find a correlation. We did, however, observe an association between slow wave slope decline and behaviour, with children who did not have behavioural problems having a steeper overnight slow wave slope decline than those with reported behavioural problems. The lack of correlation between slow wave slope decline and neuropsychological test results might be attributed to the temporal relation of sleep EEG and NPA (separated in time by up to four months), and by the type of tests used. The quantitative test results of NPA might be better used to assess the long-term developmental trajectories of children with ESES, while they are probably insensitive to short-term fluctuations that may be related to changes in ESES severity and slow wave homeostasis in the context of treatment and spontaneous fluctuations. We believe that impaired synaptic homeostasis is currently the best model to explain cognitive and behavioural disturbances in children with ESES. We recommend future studies that relate overnight slow wave course to overnight testing of recall or improvement in task performance, e.g. using a visuomotor task, similar to the test that Boly et al. used in their study of focal epilepsy patients. ${ }^{62}$

\section{Treatment}

Treatment of ESES is often difficult and treatment strategies vary widely among clinicians. ${ }^{39,63}$ We performed a systematic review and pooled analysis of published cases with encephalopathy with ESES. We found that benzodiazepines, steroids and surgery are generally more effective in improving cognitive functioning or epileptiform EEG activity as compared to conventional anti-epileptic drugs. ${ }^{64}$ The included studies were mostly retrospective and the results were probably influenced by publication bias. Several expert reviews have proposed treatment algorithms for children with encephalopathy with ESES. 
Most of these reviews suggest that benzodiazepines or steroids can be given specifically for ESES, while a conventional anti-epileptic drug can be prescribed in cases with recurrent seizures. $3,65,66$ Some report that specific anti-epileptic drugs, such as levetiracetam, valproic acid, sulthiam and ethosuximide, can also be effective in controlling the ESES EEG abnormalities and improving cognition. ${ }^{3,67}$ A very recent review article suggested that in cases with a structural unilateral aetiology, epilepsy surgery should be considered a firstline treatment, whereas in cases without a clear structural aetiology, the first-line treatment may depend on the severity of the cognitive sequelae. For children with a delay or arrest of development, they suggest that clobazam can be started first and corticosteroids can be added after 3 months if no improvement is observed. In children with a regression of cognitive abilities, they proposed that corticosteroids are initiated first and clobazam can be added if no improvement is seen. ${ }^{66}$

Monitoring the effects of treatment with repeat EEGs and assessment of cognitive functioning is of great importance. We found that subjective cognitive improvement is associated with EEG improvement. ${ }^{21}$ Quantification of cognitive functioning is preferred to provide a more precise and reproducible measure of treatment efficacy. However, neuropsychological test results appear to be relatively insensitive to fluctuations in the context of treatment, as described above. ${ }^{21}$ In addition, the tests may not be able to reveal a change in performance in the children with severe neurodevelopmental delay, with results below the lowest measurable level. Neuropsychological test results are probably useful for long-term monitoring of neurodevelopment, whereas new tools for short-term evaluation of treatment efficacy should be developed and validated. ${ }^{68}$

In the near future, we hope to prove which initial treatment for encephalopathy with ESES is most effective. For this purpose a comparative randomised controlled trial (RCT) is the gold standard. We are conducting a multicentre European RCT (acronym RESCUE ESES: Randomised European trial of Steroids vs Clobazam Usage for Encephalopathy with Electrical Status Epilepticus in Sleep). This study compares efficacy of 6 months of treatment with clobazam versus treatment with corticosteroids. As the treatments under evaluation are already widely used, the study can be labelled a pragmatic RCT. We also investigate whether inflammatory mediators can be used to predict whether one of the treatment options is probably more effective than the other.

On the longer term, future study results may help to define a therapeutic approach that implements imaging findings, inflammatory markers and genetic test results (e.g. GRIN2A mutation status) and allow precision medicine for each individual child. A patient with a clear structural lesion, in a location where resection is safe, may qualify for an early operation. In patients with ESES after perinatal thalamic injury, targeted therapy such 
as thalamic deep brain stimulation (DBS), may be an interesting topic for future studies. Randomised controlled trials have shown that thalamic DBS significantly reduces seizure frequency in patients with medically refractory epilepsy. ${ }^{69-71}$ Furthermore, studies on the influence of DBS on sleep-wake disturbances in patients with Parkinson's disease, have shown that treatment with DBS is associated with improvement in sleep quality, sleep efficiency, and sleep duration. ${ }^{72}$ In a very recent study using a mouse model of narcolepsy, a sleep disorder with disorganised sleep and wake states, DBS resulted in better sleep - wake consolidation. ${ }^{73}$ Future studies may prove whether deep brain stimulation is a safe and effective treatment in children with encephalopathy with ESES with and without thalamic injury. Unfortunately, the current lack of a well-established mouse model for ESES limits possibilities to test this treatment in a preclinical setting.

Patients with an activated inflammatory profile could be treated with (targeted) antiinflammatory treatment. A patient with a GRIN2A gain of function mutation (encoding a subunit of the NMDA-specific glutamate receptor, having a major role in excitation in the brain), may benefit from treatment that supresses the activity of the glutamate receptor. Previous reports have suggested that existing drugs targeting this receptor (e.g. memantine and amantadine) may be beneficial. ${ }^{74-76}$

\section{Pragmatic randomised controlled trials (RCTs): lessons learned from RESCUE ESES}

In 2012, we received funding, altogether 353.808 Euro, for a European randomised controlled trial (acronym: RESCUE ESES) comparing treatment with clobazam to treatment with corticosteroids in 130 children with encephalopathy with ESES. The effects of those treatments are evaluated with regular EEGs and repeated neuropsychological assessment. Predictors of outcome are assessed. The protocol for this study (described in chapter 8) was drafted by a steering committee consisting of 7 experts in the treatment of ESES from three centres across three countries. A large group of experienced paediatric neurologists, from 24 centres across Europe, acknowledged the value of the trial, had the opportunity to provide input to the study protocol, and intended to participate. The EU organisation European Clinical Research Infrastructure Network (ECRIN) endorsed their logistical support for the international extension of the study, which included preparation and submission of documents for ethics approval in countries outside the Netherlands and monitoring during the conduct of the study. In December 2013, the study protocol was approved by the UMC Utrecht ethics committee and in February 2014 by the Dutch competent authority. The first patient was included in Utrecht in July 2014. This was a promising start. 
What happened since then? Currently, 6 years later, 43 patients have been included. Initially, it was expected that by the end of 2018 the required 130 patients would have been included. Several aspects caused significant delay in the initiation of the study in the participating centres:

I. Ethics committee and competent authority approval: Despite the pragmatic design of the RESCUE ESES study, ethics committees and competent authorities in each country had to approve the study. The process of preparation of the study files involved enormous amounts of paperwork and ethics committees often asked for changes to the study protocol, whereas the study protocol had already been approved by the ethics committee of the principal investigator's (also sponsor's) site (UMC Utrecht). As a consequence, we had to submit numerous amendments to the study protocol, that each subsequently had to be submitted to all other centres to keep the protocol uniform across all study sites. The heterogeneity in procedures and requests by ethics committees and competent authorities across countries altogether caused significant delay.

II. Study medications: Despite the fact that clobazam and corticosteroids are routinely prescribed for epilepsy in the clinical setting in all participating centres, for some countries it was required to prepare and label the study medication for clinical trial use. This was a challenge to organise, involved another costly and time-consuming procedure, and medications had to be prepared for the study beforehand, whilst not knowing how many patients per centre would be included. In one country, the national drug agency required review by an external expert, who withheld approval for the study.

III. Clinical trial agreements: Most centres required changes to the clinical trial agreement that the UMC Utrecht developed for international clinical trials, and some would only accept their own clinical trial agreement template to be used. For some centres, finding a mutually acceptable agreement involved a time-consuming process of extensive negotiations between legal counsellors.

IV. Insurance requirements: Whilst some centres have standard insurance policies that cover study participants, for other centres study-specific participant insurance had to be contracted. The insurance requirements differed per country and study centre.

To date, approval was granted for the conduct of the trial in 16 centres across 8 countries. In 5 centres where preparations were initiated, the study was found not feasible for varying reasons. Altogether, the administrative (and financial) burden per required included patient, was extremely high. After the approval in the majority of participating centres, several other challenges were faced and actions undertaken: 
I. Initiation visits and annual monitoring requirements: For each centre, initiation visits and sometimes multiple visits needed to be scheduled. After initiation of the study, monitoring visits were required for each centre annually, independent of the number of inclusions.

II. Training of study personnel: Multiple training meetings for investigators, clinical trial nurses and study monitors were organised online and at international meetings.

III. Participant recruitment: Despite an expected number of inclusions of at least 4 per year for most of the centres, the inclusion rate was much lower. We tried to improve the inclusion rate by sending newsletters, announcements of new inclusions and study pocket cards. In addition, we published announcements of the trial in newsletters of the European Paediatric Neurology Society and national paediatric neurology associations. Unfortunately, the number of inclusions lagged far behind on expectations.

IV. Investigators reported that patients / parents of study candidates refrained from participating for various reasons: a perceived delay in start of treatment if they would participate in the study, a preference for one of the treatment arms, or inability to meet with study timelines for follow-up visits and investigations. In addition, some patients could not be included because they had already been treated with one of the study medications, or were not referred in time to a study site by their treating doctors.

V. The lack of available funding for local study investigators and research nurses probably led to a limited availability of local investigators and nurses for the trial and a competition with (often more urgent) routine clinical duties. In 6 centres, the principal investigator moved to another hospital, and finding a new PI was a challenge for some of these centres.

Although the abovementioned difficulties - or rather challenges - probably play a role, it is unclear why the speed of inclusions is so variable between centres, with some centres having no inclusions after 5 years, whilst others included 5 or more patients.

\section{Pragmatic randomised controlled trials: suggestions for the future}

The challenges that we faced in the conduct of the RESCUE ESES trial, have been reported by many investigators. A systematic analysis of registered trials in rare disease populations found that more than half of the trials were either discontinued or unpublished 4 years after completion.77 Similar results were found in a systematic analysis of randomised clinical trials in children, the authors of which identified that the risk of discontinuation or non-publication was highest in academic (as compared to industry-sponsored) studies.78 Several barriers to the conduct of randomised controlled trials (especially in rare disease populations) and suggested solutions have been reported in the literature, which is summarised in table 1.79-81 
For most of the abovementioned challenges, solutions were found for the RESCUE ESES trial. Whilst we still strive to achieve the required number of inclusions for RESCUE ESES, we realize that the trial may have to be discontinued earlier for reasons of feasibility.

Table 1. Challenges in the conduct of randomised controlled trials in rare disease populations

\begin{tabular}{|c|c|c|}
\hline & Barriers & Suggested solutions \\
\hline Research question and outcome measures & $\begin{array}{l}\text { Limited literature available to determine } \\
\text { research question; outcome measures } \\
\text { insensitive }\end{array}$ & $\begin{array}{l}\text { Prepare study in a multicentre / } \\
\text { international team of experts; choose } \\
\text { outcome measure that is sensitive }\end{array}$ \\
\hline Inadequate funding & $\begin{array}{l}\text { Academic trials often do not have sufficient } \\
\text { funding to cover all research procedures }\end{array}$ & $\begin{array}{l}\text { Establish a support frame work for rare } \\
\text { disease studies }\end{array}$ \\
\hline Inadequate understanding of methodology & $\begin{array}{l}\text { Trials often designed by clinicians, having } \\
\text { limited methodological background }\end{array}$ & $\begin{array}{l}\text { Provide adequate research support for } \\
\text { clinicians designing a trial, including early } \\
\text { consultation of a methodologist }\end{array}$ \\
\hline Study population & $\begin{array}{l}\text { Due to rarity, multicentre and international } \\
\text { collaboration required to recruit enough } \\
\text { patients }\end{array}$ & Thematic collaborative research networks \\
\hline Regulatory requirements & $\begin{array}{l}\text { Complex regulatory requirements; separate } \\
\text { approval needed for each country }\end{array}$ & $\begin{array}{l}\text { Harmonise regulations globally, or at } \\
\text { least within Europe, and implement } \\
\text { internationally recognised review bodies }\end{array}$ \\
\hline Excessive monitoring & $\begin{array}{l}\text { Monitoring requirements are independent } \\
\text { of trial type and excessive for rare diseases }\end{array}$ & $\begin{array}{l}\text { Establish different monitoring regulations } \\
\text { for industry sponsored and academic } \\
\text { studies; define requirements according } \\
\text { to number of included patients instead of } \\
\text { annually. }\end{array}$ \\
\hline
\end{tabular}

\section{Concluding remarks}

We investigated several aspects of encephalopathy with ESES and gained new insights in the aetiology, pathophysiology and treatment of this severe paediatric epilepsy syndrome. Hopefully, the findings presented in this thesis and the ongoing RESCUE ESES trial, will provide an important step towards early recognition, individualised and effective treatment and a better neurodevelopmental outcome in children with encephalopathy with ESES. Lessons learned from our European randomised controlled trial will hopefully help in the design and conduct of future trials. We also hope that regulatory authorities will harmonise regulations across countries and tailor regulations to rare disease populations, which will aid in the discovery of much needed effective treatments. 


\section{References}

1. Van Hirtum-Das M, Licht EA, Koh S, Wu JY, Donald Shields W, Sankar R. Children with ESES: Variability in the syndrome. Epilepsy Res. 2006;70(SUPPL.1):248-258. doi:10.1016/j.eplepsyres.2006.01.020

2. Sánchez Fernández I, Loddenkemper T, Peters JM, Kothare S V. Electrical status epilepticus in sleep: Clinical presentation and pathophysiology. Pediatr Neurol. 2012;47(6):390-410. doi:10.1016/j. pediatrneurol.2012.06.016

3. Nickels K, Wirrell E. Electrical Status Epilepticus in Sleep. Semin Pediatr Neurol. 2008;15(2):50-60. doi:10.1016/j.spen.2008.03.002

4. Giovanardi Rossi P, Parmeggiani A, Posar A, Scaduto MC, Chiodo S, Vatti G. Landau-Kleffner syndrome (LKS): long-term follow-up and links with electrical status epilepticus during sleep (ESES). Brain Dev. 1999;21(2):90-98. doi:10.1016/S0387-7604(98)00071-0

5. Silvestri R, Gagliano A, Calarese T, et al. Ictal and interictal EEG abnormalities in ADHD children recorded over night by video-polysomnography. Epilepsy Res. 2007;75(2-3):130-137. doi:10.1016/j. eplepsyres.2007.05.007

6. Patry G, Lyagoubi S, Tassinari CA. Subclinical "electrical status epilepticus"induced by sleep in children. Arch Neurol. 1971;24(3):242-252. doi:http://dx.doi.org/10.1001/archneur.1971.00480330070006

7. Commission on Classification and Terminology of the International League Against Epilepsy. Proposal for Revised Classification of Epilepsies and Epileptic Syndromes. Epilepsia. 1989;30(4):389399. doi:10.1111/j.1528-1157.1989.tb05316

8. Tassinari C a., Rubboli G, Volpi L, et al. Encephalopathy with electrical status epilepticus during slow sleep or ESES syndrome including the acquired aphasia. Clin Neurophysiol. 2000;111(SUPPL. 2):94102. doi:10.1016/S1388-2457(00)00408-9

9. Scheltens-De Boer M. Guidelines for EEG in encephalopathy related to ESES/CSWS in children. In: Epilepsia. Vol 50. ; 2009:13-17. doi:10.1111/j.1528-1167.2009.02211

10. Fernández IS, Chapman KE, Peters JM, et al. The tower of Babel: Survey on concepts and terminology in electrical status epilepticus in sleep and continuous spikes and waves during sleep in North America. Epilepsia. 2013;54(4):741-750. doi:10.1111/epi.12039

11. Galer S, Urbain C, De Tiège $X$, et al. Impaired sleep-related consolidation of declarative memories in idiopathic focal epilepsies of childhood. Epilepsy Behav. 2015;43:16-23. doi:10.1016/j. yebeh.2014.11.032

12. Kersbergen KJ, De Vries LS, Leijten FSS, et al. Neonatal thalamic hemorrhage is strongly associated with electrical status epilepticus in slow wave sleep. Epilepsia. 2013;54(4):733-740. doi:10.1111/ epi.12131

13. Bartolini E, Falchi M, Zellini F, et al. The syndrome of polymicrogyria, thalamic hypoplasia, and epilepsy with CSWS. Neurology. 2016;86(13):1250-1259. doi:10.1212/WNL.00000000000002526

14. Lemke JR, Lal D, Reinthaler EM, et al. Mutations in GRIN2A cause idiopathic focal epilepsy with rolandic spikes. Nat Genet. 2013;45(9):1067-1072. doi:10.1038/ng.2728

15. Saltik S, Uluduz D, Cokar O, Demirbilek V, Dervent A. A clinical and EEG study on idiopathic partial epilepsies with evolution into ESES spectrum disorders. Epilepsia. 2005;46(4):524-533. doi:10.1111 /j.0013-9580.2005.45004

16. Caraballo RH, Cersósimo RO, Fortini PS, et al. Congenital hemiparesis, unilateral polymicrogyria and epilepsy with or without status epilepticus during sleep: A study of 66 patients with long-term follow-up. Epileptic Disord. 2013. doi:10.1684/epd.2013.0612

17. van den Munckhof B, Zwart AF, Weeke LC, et al. Perinatal thalamic injury: MRI predictors of electrical status epilepticus in sleep and long-term neurodevelopment. Neurolmage Clin. 2020. doi:10.1016/j. nicl.2020.102227

18. Scholtes FBJ, Hendriks MPH, Renier WO. Cognitive deterioration and electrical status epilepticus during slow sleep. Epilepsy Behav. 2005;6(2):167-173. doi:10.1016/j.yebeh.2004.11.001 
19. Pera MC, Brazzo D, Altieri N, Balottin U, Veggiotti P. Long-term evolution of neuropsychological competences in encephalopathy with status epilepticus during sleep: A variable prognosis. Epilepsia. 2013;54(SUPPL.7):77-85. doi:10.1111/epi.12313

20. Tassinari CA, Rubboli G. Cognition and paroxysmal EEG activities: From a single spike to electrical status epilepticus during sleep. Epilepsia. 2006;47(SUPPL. 2):40-43. doi:10.1111/j.1528-1167.2006.00686

21. van den Munckhof B, Alderweireld C, Davelaar S, et al. Treatment of electrical status epilepticus in sleep: Clinical and EEG characteristics and response to 147 treatments in 47 patients. Eur J Paediatr Neurol. 2018;22(1):64-71. doi:10.1016/j.ejpn.2017.08.006

22. Varadkar S, Bien CG, Kruse CA, et al. Rasmussen's encephalitis: Clinical features, pathobiology, and treatment advances. Lancet Neurol. 2014. doi:10.1016/S1474-4422(13)70260-6

23. Dalmau J, Gleichman AJ, Hughes EG, et al. Anti-NMDA-receptor encephalitis: case series and analysis of the effects of antibodies. Lancet Neurol. 2008;7:1091-1098. doi:10.1016/S1474-4422(08)70224-2

24. Dalmau J, Lancaster E, Martinez-Hernandez E, Rosenfeld MR, Balice-Gordon R. Clinical experience and laboratory investigations in patients with anti-NMDAR encephalitis. Lancet Neurol. 2011. doi:10.1016/S1474-4422(10)70253-2

25. Titulaer MJ, McCracken L, Gabilondo I, et al. Treatment and prognostic factors for long-term outcome in patients with anti-NMDA receptor encephalitis: An observational cohort study. Lancet Neurol. 2013. doi:10.1016/S1474-4422(12)70310-1

26. Korff CM, Dale RC. The immune system in pediatric seizures and epilepsies. Pediatrics. 2017. doi:10.1542/peds.2016-3534

27. Graus F, Titulaer MJ, Balu R, et al. A clinical approach to diagnosis of autoimmune encephalitis. Lancet Neurol. 2016. doi:10.1016/S1474-4422(15)00401-9

28. de Vries EE, van den Munckhof B, Braun KPJ, van Royen-Kerkhof A, de Jager W, Jansen FE. Inflammatory mediators in human epilepsy: A systematic review and meta-analysis. Neurosci Biobehav Rev. 2016;63:177-190. doi:10.1016/j.neubiorev.2016.02.007

29. Vezzani A, Viviani B. Neuromodulatory properties of inflammatory cytokines and their impact on neuronal excitability. Neuropharmacology. 2015;96(Pt A):70-82. doi:10.1016/j. neuropharm.2014.10.027

30. Auvin S, Mazarati A, Shin D, Sankar R. Inflammation enhances epileptogenesis in the developing rat brain. Neurobiol Dis. 2010;40(1):303-310. doi:10.1016/j.nbd.2010.06.004

31. Kalueff A V, Lehtimaki KA, Ylinen A, Honkaniemi J, Peltola J. Intranasal administration of human IL-6 increases the severity of chemically induced seizures in rats. Neurosci Lett. 2004;365(2):106-110. doi:10.1016/j.neulet.2004.04.061

32. Dubé C, Vezzani A, Behrens M, Bartfai T, Baram TZ. Interleukin-1 $\beta$ contributes to the generation of experimental febrile seizures. Ann Neurol. 2005;57(1):152-155. doi:10.1002/ana.20358

33. Vezzani A, Balosso S, Ravizza T. Neuroinflammatory pathways as treatment targets and biomarkers in epilepsy. Nat Rev Neurol. 2019. doi:10.1038/s41582-019-0217

34. Buzatu M, Bulteau C, Altuzarra C, Dulac O, Van Bogaert P. Corticosteroids as treatment of epileptic syndromes with continuous spike-waves during slow-wave sleep. Epilepsia. 2009;50:68-72. doi:10.1 111/j.1528-1167.2009.02224

35. Sinclair DB, Snyder TJ. Corticosteroids for the treatment of Landau-kleffner syndrome and continuous spike-wave discharge during sleep. Pediatr Neurol. 2005;32(5):300-306. doi:10.1016/j. pediatrneurol.2004.12.006

36. Kramer U, Sagi L, Goldberg-Stern H, Zelnik N, Nissenkorn A, Ben-Zeev B. Clinical spectrum and medical treatment of children with electrical status epilepticus in sleep (ESES). Epilepsia. 2009;50(6):15171524. doi:10.1111/j.1528-1167.2008.01891

37. Mikati MA, Saab R, Fayad MN, Choueiri RN. Efficacy of intravenous immunoglobulin in LandauKleffner syndrome. Pediatr Neurol. 2002. doi:10.1016/S0887-8994(01)00402-7 
Chapter 10. General discussion

38. Arts WFM, Aarsen FK, Scheltens-De Boer M, Catsman-Berrevoets CE. Landau-Kleffner syndrome and CSWS syndrome: Treatment with intravenous immunoglobulins. In: Epilepsia. ; 2009. doi:10.1111 /j.1528-1167.2009.02221

39. Van Den Munckhof B, Van Dee V, Sagi L, et al. Treatment of electrical status epilepticus in sleep: A pooled analysis of 575 cases. Epilepsia. 2015;56(11):1738-1746. doi:10.1111/epi.13128

40. Lehtimaki KA, Liimatainen S, Peltola J, Arvio M. The serum level of interleukin-6 in patients with intellectual disability and refractory epilepsy. Epilepsy Res. 2011;95(1-2):184-187. doi:10.1016/j. eplepsyres.2011.03.004 [doi]

41. Rutz S, Wang X, Ouyang W. The IL-20 subfamily of cytokines-from host defence to tissue homeostasis. Nat Rev Immunol. 2014. doi:10.1038/nri3766

42. Fabene PF, Bramanti P, Constantin G. The emerging role for chemokines in epilepsy. J Neuroimmunol. 2010. doi:10.1016/j.jneuroim.2010.05.016

43. Cerri C, Caleo M, Bozzi Y. Chemokines as new inflammatory players in the pathogenesis of epilepsy. Epilepsy Res. 2017. doi:10.1016/j.eplepsyres.2017.07.016

44. Hancock EC, Osborne JP, Edwards SW. Treatment of infantile spasms. Cochrane database Syst Rev. 2013;6:CD001770. doi:10.1002/14651858.CD001770.pub3

45. Rhen T, Cidlowski JA. Antiinflammatory action of glucocorticoids - New mechanisms for old drugs. NEngl J Med. 2005. doi:10.1056/NEJMra050541

46. Bialer M, Johannessen SI, Levy RH, Perucca E, Tomson T, White HS. Progress report on new antiepileptic drugs: A summary of the Eleventh Eilat Conference (EILAT XI). Epilepsy Res. 2013. doi:10.1016/j.eplepsyres.2012.10.001

47. Berg AT, Berkovic SF, Brodie MJ, et al. Revised terminology and concepts for organization of seizures and epilepsies: Report of the ILAE Commission on Classification and Terminology, 2005-2009. Epilepsia. 2010;51(4):676-685. doi:10.1111/j.1528-1167.2010.02522

48. Scheffer IE, Berkovic S, Capovilla G, et al. ILAE classification of the epilepsies: Position paper of the ILAE Commission for Classification and Terminology. Epilepsia. 2017. doi:10.1111/epi.13709

49. Huber R, Felice Ghilardi M, Massimini M, Tononi G. Local sleep and learning. Nature. 2004;430(6995):7881. doi:10.1038/nature02663

50. Tononi G, Cirelli C. Sleep function and synaptic homeostasis. Sleep Med Rev. 2006;10(1):49-62. doi:10.1016/j.smrv.2005.05.002

51. Tononi G, Cirelli C. Sleep and the Price of Plasticity: From Synaptic and Cellular Homeostasis to Memory Consolidation and Integration. Neuron. 2014;81(1):12-34. doi:10.1016/j.neuron.2013.12.025

52. De Vivo L, Bellesi M, Marshall W, et al. Ultrastructural evidence for synaptic scaling across the wake/ sleep cycle. Science (80- ). 2017;355(6324):507-510. doi:10.1126/science.aah5982

53. Vyazovskiy $\vee$ V., Olcese U, Lazimy YM, et al. Cortical Firing and Sleep Homeostasis. Neuron. 2009;63(6):865-878. doi:10.1016/j.neuron.2009.08.024

54. Esser SK, Hill SL, Tononi G. Sleep homeostasis and cortical synchronization: I. Modeling the effects of synaptic strength on sleep slow waves. Sleep. 2007;30(12):1617-1630.

55. Vyazovskiy V V, Riedner B a, Cirelli C, Tononi G. Sleep homeostasis and cortical synchronization: II. A local field potential study of sleep slow waves in the rat. Sleep. 2007;30(12):1631-1642. doi:18246974

56. Riedner B a, Vyazovskiy VV, Huber R, et al. Sleep homeostasis and cortical synchronization: III. A highdensity EEG study of sleep slow waves in humans. Sleep. 2007;30(12):1643-1657. doi:18246974

57. Bölsterli BK, Schmitt B, Bast T, et al. Impaired slow wave sleep downscaling in encephalopathy with status epilepticus during sleep (ESES). Clin Neurophysiol. 2011;122(9):1779-1787. doi:10.1016/j. clinph.2011.01.053

58. Bölsterli Heinzle BK, Fattinger S, Kurth S, et al. Spike wave location and density disturb sleep slow waves in patients with CSWS (continuous spike waves during sleep). Epilepsia. 2014;55(4):584-591. doi:10.1111/epi.12576 
59. Bölsterli BK, Gardella E, Pavlidis E, et al. Remission of encephalopathy with status epilepticus (ESES) during sleep renormalizes regulation of slow wave sleep. Epilepsia. 2017;58(11):1892-1901. doi:10.1111/epi.13910

60. Steriade M, Amzica F. Dynamic coupling among neocortical neurons during evoked and spontaneous spike-wave seizure activity. J Neurophysiol. 1994. doi:10.1152/jn.1994.72.5.2051

61. Rubboli G, Huber R, Tononi G, Tassinari CA. Encephalopathy related to Status Epilepticus during slow Sleep: a link with sleep homeostasis? Epileptic Disord. 2019. doi:10.1684/epd.2019.1059

62. Boly M, Jones B, Findlay $G$, et al. Altered sleep homeostasis correlates with cognitive impairment in patients with focal epilepsy. Brain. 2017;140(4):1026-1040. doi:10.1093/brain/awx017

63. Sánchez Fernández I, Chapman K, Peters JM, et al. Treatment for continuous spikes and waves during sleep (CSWS): Survey on treatment choices in North America. Epilepsia. 2014;55:1099-1108. doi:10.1111/epi.12678

64. van den Munckhof B, van Dee V, Sagi L, et al. Treatment of electrical status epilepticus in sleep: A pooled analysis of 575 cases. Epilepsia. 2015;56(11):1738-1746. doi:10.1111/epi.13128

65. Veggiotti P, Pera MC, Teutonico F, Brazzo D, Balottin U, Tassinari CA. Therapy of encephalopathy with status epilepticus during sleep (ESES/CSWS syndrome): An update. Epileptic Disord. 2012;14(1):1-11. doi:10.1684/epd.2012.0482

66. Jansen FE, Nikanorova M, Peltola M. Current treatment options for Encephalopathy related to Status Epilepticus during slow Sleep. Epileptic Disord. 2019. doi:10.1684/epd.2019.1061

67. Fernández IS, Chapman KE, Peters JM, Harini C, Rotenberg A, Loddenkemper T. Continuous Spikes and Waves during Sleep: Electroclinical Presentation and Suggestions for Management. Epilepsy Res Treat. 2013;2013:583531. doi:10.1155/2013/583531

68. Arzimanoglou A, Cross HJ. Cognitive impairment and behavioral disorders in Encephalopathy related to Status Epilepticus during slow Sleep: diagnostic assessment and outcome. Epileptic Disord. 2019. doi:10.1684/epd.2019.1060

69. Sprengers M, Vonck K, Carrette E, Marson AG, Boon P. Deep brain and cortical stimulation for epilepsy. Cochrane Database Syst Rev. 2017. doi:10.1002/14651858.CD008497.pub3

70. Bouwens van der Vlis TAM, Schijns OEMG, Schaper FLWVJ, et al. Deep brain stimulation of the anterior nucleus of the thalamus for drug-resistant epilepsy. Neurosurg Rev. 2019. doi:10.1007/s10143-0170941

71. Li MCH, Cook MJ. Deep brain stimulation for drug-resistant epilepsy. Epilepsia. 2018. doi:10.1111/ epi.13964

72. Sharma VD, Sengupta S, Chitnis S, Amara AW. Deep brain stimulation and sleep-wake disturbances in parkinson disease: A review. Front Neurol. 2018. doi:10.3389/fneur.2018.00697

73. Rogers AA, Aiani LM, Blanpain LT, Yuxian S, Moore R, Willie JT. Deep Brain Stimulation of Hypothalamus for Narcolepsy-Cataplexy in Mice. Brain Stimul. 2020. doi:10.1016/j.brs.2020.04.006

74. Pierson TM, Yuan H, Marsh ED, et al. GRIN2A mutation and early-onset epileptic encephalopathy: personalized therapy with memantine. Ann Clin Trans/ Neurol. 2014. doi:10.1002/acn3.39

75. Perucca P, Perucca E. Identifying mutations in epilepsy genes: Impact on treatment selection. Epilepsy Res. 2019. doi:10.1016/j.eplepsyres.2019.03.001

76. Wilson RB, Eliyan Y, Sankar R, Hussain SA. Amantadine: A new treatment for refractory electrical status epilepticus in sleep. Epilepsy Behav. 2018;84:74-78. doi:10.1016/j.yebeh.2018.04.018

77. Rees CA, Pica N, Monuteaux MC, Bourgeois FT. Noncompletion and nonpublication of trials studying rare diseases: A cross-sectional analysis. PLoS Med. 2019. doi:10.1371/journal.pmed.1002966

78. Pica N, Bourgeois F. Discontinuation and nonpublication of randomized clinical trials conducted in children. Pediatrics. 2016. doi:10.1542/peds.2016-0223

79. Rath A, Salamon V, Peixoto S, et al. A systematic literature review of evidence-based clinical practice for rare diseases: What are the perceived and real barriers for improving the evidence and how can they be overcome? Trials. 2017. doi:10.1186/s13063-017-2287-7 
Chapter 10. General discussion

80. Ford I, Norrie J. Pragmatic trials. N Engl J Med. 2016. doi:10.1056/NEJMra1510059

81. Djurisic S, Rath A, Gaber S, et al. Barriers to the conduct of randomised clinical trials within all disease areas. Trials. 2017. doi:10.1186/s13063-017-2099-9 

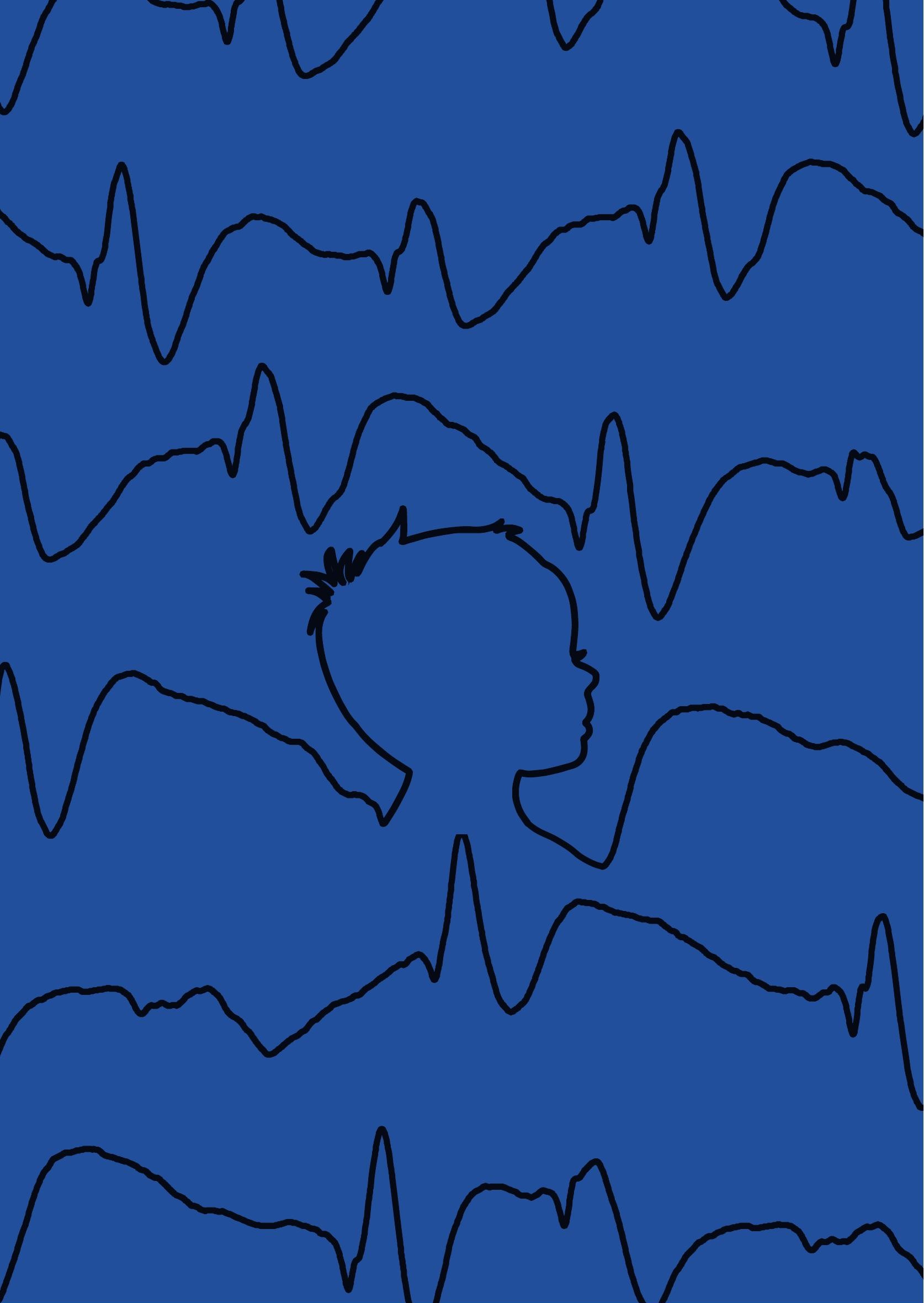
Appendix 


\section{Nederlandstalige samenvatting | Summary in Dutch}

Encefalopathie met elektrische status epilepticus in slaap (ESES), of "ESES syndroom", is een zeldzaam epilepsiesyndroom dat bij kinderen kan voorkomen vanaf 2 jaar. Kinderen met het ESES syndroom hebben in slaap vrijwel continue epileptische activiteit zonder dat dit zichtbaar is bij het kind. Deze vorm van epilepsie kan worden vastgesteld met een slaap elektro-encefalogram (EEG), een registratie van de hersenactiviteit in slaap. Wanneer er gedurende meer dan 85\% van de tijd epileptische activiteit wordt gezien (een "piekgolfindex" van >85\%), wordt dit "klassiek ESES syndroom" genoemd. Bij een piekgolfindex van 50-85\% gaat het om "atypisch ESES syndroom". Een groot deel van de kinderen met ESES syndroom heeft ook af en toe een zichtbare epileptische aanval.

De vrijwel continue epileptische activiteit in slaap gaat gepaard met problemen van de ontwikkeling van het kind, zoals leer- en gedragsproblemen. Soms verliezen kinderen met ESES syndroom hun taalvaardigheid, een variant die het "Landau-Kleffner syndroom" wordt genoemd. Bij andere kinderen gaan de schoolprestaties achteruit of staan gedragsproblemen op de voorgrond. De vrijwel continue epileptische activiteit in slaap verdwijnt in de puberteit, maar de opgelopen leerachterstand is vaak blijvend. Bij een derde tot de helft van de kinderen met ESES syndroom wordt een structurele afwijking van de hersenen gevonden, bijvoorbeeld een aanlegstoornis van de hersenschors of een beschadiging die rond de geboorte is ontstaan. Soms wordt een genetische oorzaak gevonden, zoals een GRIN2A mutatie. Bij ongeveer de helft van de kinderen met ESES syndroom wordt geen oorzaak gevonden.

Het vroegtijdig stellen van de diagnose en het vlot starten van behandeling kan ernstige gevolgen voorkomen of verminderen. Het is dus van groot belang om te weten wanneer aan ESES syndroom moet worden gedacht en welke kinderen een verhoogd risico lopen op het krijgen van dit epilepsiesyndroom. De behandeling van ESES syndroom wordt bemoeilijkt doordat normale anti-epileptische medicijnen (reguliere anti-epileptica) vaak een beperkt effect hebben op de epilepsie in slaap. Benzodiazepines (zoals het middel clobazam) en corticosteroïden (ontstekingsremmers) worden ook vaak gegeven aan kinderen met ESES syndroom. Bij kinderen met een structurele afwijking van een beperkt deel van de hersenen, kan een hersenoperatie worden overwogen.

Het doel van dit proefschrift was om: 1) handvatten te bieden voor de vroegtijdige herkenning van kinderen met een verhoogd risico op het ontwikkelen van ESES, 2) meer inzicht te verkrijgen in de oorzaken van ESES en hoe ESES leidt tot leer- en gedragsproblemen en 3) meer wetenschappelijk bewijs te vinden voor wat de optimale 
behandeling is voor kinderen met het ESES syndroom. We hebben verschillende aspecten van het ESES syndroom bestudeerd, welke hieronder zijn samengevat.

\section{Deel I. Oorzaken en verklarende mechanismen}

In hoofdstuk 2 beschrijven we een groep van 30 kinderen die rond de geboorte een beschadiging opliepen van de thalamus, een belangrijk regelcentrum in de hersenen. Deze kinderen werden gevolgd met slaap EEG's vanaf 2 jaar en hun ontwikkeling werd gevolgd met neuropsychologische onderzoeken. We lieten zien dat het merendeel van de kinderen, die rond de geboorte thalamusletsel opliepen, vanaf de leeftijd van 2 jaar ESES krijgt. Het resterende volume van de thalamus, gemeten op een MRI scan na 3 maanden, bleek te voorspellen hoe hun ontwikkeling op de lange termijn verloopt: kinderen met een groter resterend thalamusvolume, hadden op de lange termijn hogere totaal IQ scores. Ook onderzochten we met "diffusion tensor imaging" (DTI), een techniek om de zenuwbanen in de hersenen te beoordelen, of een verstoring van de structuur van deze zenuwbanen het optreden van ontwikkelingsproblemen kan verklaren. We vonden dat een relatief eenduidige richting van de zenuwbanen in de thalamus op de kinderleeftijd, geassocieerd is met een hoger totaal IQ. Dit suggereert dat het hebben van een relatief normale structuur van de resterende thalamus gunstig is voor de ontwikkeling van de hersenen bij kinderen met een beschadiging van de thalamus die is ontstaan rond de geboorte.

In hoofdstuk 3 presenteren we de resultaten van een systematische literatuurstudie en meta-analyse van studies naar ontstekingseiwitten bij mensen met epilepsie. We vonden dat verschillende ontstekingseiwitten met een brede functie (zoals interleukine 6, IL-6) verhoogd zijn bij mensen met epilepsie en dat bij verschillende vormen van epilepsie bepaalde patronen in de activatie van deze eiwitten te onderscheiden zijn. Ook onderzochten we ontstekingseiwitten in verschillende lichaamsmaterialen, namelijk in bloed, liquor (hersenvocht) en in hersenweefsel van geopereerde epilepsie patiënten. In hersenweefsel bleek een specifieke groep ontstekingseiwitten, de chemokines, in het bijzonder verhoogd te zijn. Dit suggereert dat er bij mensen met epilepsie sprake is van verhoogde ontstekingsactiviteit in het gehele lichaam, maar daarnaast van een specifiekere ontstekingsreactie in de hersenen. Toekomstige studies kunnen mogelijk verhelderen of de verhoogde ontstekingsactiviteit een oorzakelijke rol heeft, of slechts een gevolg is van het hebben van epileptische aanvallen. Ook kan de rol van behandeling met ontstekingsremmende medicijnen verder worden onderzocht. 
In hoofdstuk 4 onderzochten we of de hoeveelheid van ontstekingseiwitten in het bloed van kinderen met het ESES syndroom verhoogd is in vergelijking met controles zonder epilepsie. Van de 16 ontstekingseiwitten die we onderzochten, waren er 7 significant verschillend tussen de twee groepen: 5 ontstekingseiwitten waren hoger en 2 waren er juist lager bij kinderen met het ESES syndroom. We vonden ook dat de hoeveelheid ontstekingseiwit IL-6 significant daalde na behandeling met ontstekingsremmers en dat dit samenging met een afname van de epileptische afwijkingen op het EEG en met een verbetering van de neuropsychologische test resultaten. We veronderstellen dat de activiteit van verschillende ontstekingseiwitten gerelateerd is aan de ernst van het ESES syndroom en speculeren dat, tenminste bij een deel van de kinderen met ESES syndroom, ontstekingsremmers een gunstig effect kunnen hebben.

In hoofdstuk 5 bestudeerden we of ontwikkelings- en gedragsproblemen bij kinderen met ESES syndroom kunnen worden verklaard door een verstoring van de normale slaapritmes. Een mogelijke verklaring voor deze problemen, hebben we gezocht vanuit de "synaptische homeostase hypothese". Deze hypothese beschrijft dat er bij het wakker zijn nieuwe verbindingen (synapsen) tussen hersencellen worden gevormd en dat bestaande verbindingen worden versterkt ("synaptische vermeerdering"). Hierdoor worden nieuwe ervaringen en nieuwe kennis vastgelegd. Ter compensatie wordt in slaap een deel van de verbindingen opgeheven en neemt de sterkte van andere verbindingen af ("synaptische vermindering"). Door deze synaptische vermindering blijft het hersennetwerk compact en efficiënt en is er na een nacht slaap weer ruimte om nieuwe ervaringen en informatie te onthouden. De synaptische homeostase is in het EEG te herkennen aan de deltagolven (trage hersengolven), een kenmerk van diepe slaap. Bij gezonde kinderen zijn deze deltagolven aan het begin van de nacht slaap erg groot en neemt het formaat van deze golven in de loop van de nacht slaap af. Een eerdere studie toonde dat deze afname bij kinderen met ESES syndroom minder of niet platsvindt. Wij vonden dat deze verstoring van de synaptische vermindering het ernstigst is op de plek waar de epilepsie het hevigst is, wat suggereert dat de epileptische activiteit bij kinderen met ESES syndroom de synaptische homeostase verstoort. Met dezelfde methode vonden we dat kinderen met gedragsproblemen een ernstigere verstoring van de synaptische vermindering in slaap hadden dan kinderen zonder gedragsproblemen. We vonden geen relatie tussen de mate van verstoring van de synaptische vermindering en de testresultaten van neuropsychologisch onderzoek. 


\section{Deel II. Behandeling}

In hoofdstuk $\mathbf{6}$ beschrijven we een systematische literatuurstudie naar de behandeling van het ESES syndroom. We hebben daarin de resultaten van 112 publicaties, waarin 575 patiënten beschreven zijn, samengevoegd in een dataset. 29 auteurs hebben op onze uitnodiging extra informatie over deze patiënten aangeleverd, waarna we 950 behandelingen in deze patiëntengroep konden analyseren. We vonden dat een vorm van verbetering, dat wil zeggen van de EEG verschijnselen of van het functioneren van het kind, werd gerapporteerd bij 49\% van de patiënten na behandeling met reguliere antiepileptica. Bij behandeling met benzodiazepines, corticosteroïden en epilepsiechirurgie werd een vorm van verbetering gezien bij respectievelijk 68\%, 81\% en 90\% van de patiënten. Om een betrouwbaardere uitspraak te kunnen doen over het behandeleffect, hebben we een subgroep analyse verricht van de grotere studies die alle achtereenvolgende patiënten met ESES syndroom in een bepaalde periode beschreven. Deze studies geven een betrouwbaarder beeld dan studies waarin slechts een of enkele patiënten beschreven werden (bijvoorbeeld vanwege een uitzonderlijk goed behandeleffect). In deze subgroep analyse werd bij een aanzienlijk kleiner deel van de kinderen succesvolle behandeling gerapporteerd voor reguliere anti-epileptica en benzodiazepines, terwijl corticosteroïden en epilepsie chirurgie nog altijd tamelijk hoge succespercentages kenden. We zochten ook naar andere voorspellers van succesvolle behandeling en vonden dat kinderen die al voordat de diagnose ESES gesteld werd een afwijkende ontwikkeling hadden, een kleinere kans op succesvolle behandeling hebben. Vanwege de veelal retrospectieve en kleine studies die in deze analyse werden meegenomen, biedt de analyse geen bewijs welke behandeling de beste is, maar slechts aanwijzingen. Toch suggereren de resultaten van deze studie dat behandeling met corticosteroïden en epilepsiechirurgie bij kinderen met ESES syndroom relatief effectief zijn.

In hoofdstuk 7 onderzochten we of verbetering van de EEG-afwijkingen na behandeling, d.w.z. een afname van de piekgolfindex, geassocieerd is met verbetering van het functioneren van kinderen met ESES syndroom. In dit onderzoek analyseerden we 147 behandelingen bij 47 patiënten uit het UMC Utrecht. Een afname van de piekgolfindex na behandeling was geassocieerd met subjectieve verbetering van het functioneren (oordeel ouders, eventueel leraar en behandelend arts), terwijl er geen significante relatie was met verandering van totaal IQ. Tevens vonden we dat behandeling met corticosteroïden (ontstekingsremmers) en hogere leeftijd bij het stellen van de diagnose geassocieerd zijn met verbetering in functioneren na behandeling. Deze resultaten kunnen helpen bij de keuze tussen verschillende behandelingen en bij de interpretatie van bevindingen na de start van een nieuwe behandeling bij kinderen met ESES syndroom. 
In hoofdstuk 8 beschrijven we de opzet van een Europese lotingsstudie (acroniem: RESCUE ESES) waarin we behandeling met clobazam (een benzodiazepine) vergelijken met behandeling met corticosterö̈den (ontstekingsremmers) bij kinderen met ESES syndroom. Het doel van de behandeling is het cognitief functioneren (de ontwikkeling) te verbeteren. We willen aan dit onderzoek 130 patiënten met de leeftijd van 2 tot 12 jaar laten deelnemen. Na loting worden zij gedurende 6 maanden behandeld met een van de twee behandelingen. De belangrijkste uitkomst is het cognitief functioneren na 6 maanden. Daarnaast beoordelen we ook het cognitief functioneren na 18 maanden, het al dan niet verbeteren van de EEG-afwijkingen, bijwerkingen en het voorkomen van epileptische aanvallen. We kijken ook of we het effect van de behandeling kunnen voorspellen met meting van de hoeveelheid ontstekingseiwitten in bloed van de deelnemers. Vanwege de zeldzaamheid van het ESES syndroom, hebben we deze studie in 24 Europese centra opgezet. Dit bracht veel uitdagingen met zich mee. Het verkrijgen van ethische goedkeuring in ieder land (met variatie in regelgeving), onderhandeling over studie-contracten en verzekeringen kostte veel tijd. Het werven van studie-deelnemers verloopt trager dan voorspeld, waarvoor diverse oorzaken zijn aan te wijzen. De eerste patiënt startte met de studie in juli 2014 en de studie loopt nog.

Samenvattend hebben de studies in dit proefschrift meer inzicht gegeven in verschillende aspecten van het ESES syndroom. In de discussie van dit proefschrift geef ik een toekomstvisie waarin de verworven inzichten helpen bij de tijdige opsporing en vroegtijdige behandeling van dit ernstige epilepsiesyndroom bij kinderen. Hopelijk vormen de resultaten van dit proefschrift, en in de nabije toekomst de resultaten van de RESCUE ESES studie, een belangrijke stap in de vroege herkenning van het ESES syndroom en effectieve "gepersonaliseerde behandeling" en leidt deze tot een verbeterde ontwikkeling van kinderen met het ESES syndroom. 


\section{List of publications}

van den Munckhof $\mathbf{B}^{*}$, van Dee $\mathrm{V}^{*}$, Sagi $L$, Caraballo $\mathrm{RH}$, Veggiotti $P$, Liukkonen $E$, Loddenkemper T, Sánchez Fernández I, Buzatu M, Bulteau C, Braun KP, Jansen FE. Treatment of electrical status epilepticus in sleep: A pooled analysis of 575 cases. Epilepsia. 2015 Nov;56(11):1738-46.

van den Munckhof B*, de Vries EE*, Braun KP, Boss HM, Willemsen MA, van Royen-Kerkhof A, de Jager W, Jansen FE. Serum inflammatory mediators correlate with disease activity in electrical status epilepticus in sleep (ESES) syndrome. Epilepsia. 2016 Feb;57(2):e45-50.

de Vries EE*, van den Munckhof B*, Braun KP, van Royen-Kerkhof A, de Jager W, Jansen FE. Inflammatory mediators in human epilepsy: A systematic review and meta-analysis. Neuroscience \& Biobehavioral Reviews. 2016 Apr;63:177-90.

van den Munckhof B, Alderweireld C, Davelaar S, van Teeseling HC, Nikolakopoulos S, Braun KPJ, Jansen FE. Treatment of electrical status epilepticus in sleep: Clinical and EEG characteristics and response to 147 treatments in 47 patients. European Journal of Paediatric Neurology. 2018 Jan;22(1):64-71.

Smit M, Van Stralen HE, Van den Munckhof B, Snijders TJ, Dijkerman HC. The man who lost his body: Suboptimal multisensory integration yields body awareness problems after a right temporoparietal brain tumour. Journal of Neuropsychology. 2018 Mar 12.

van Westrhenen A, Cats EA, van den Munckhof B, van der Salm SMA, Teunissen NW, Ferrier CH, Leijten FSS, Geleijns KPW. Specific EEG markers in POLG1 Alpers'syndrome. Clinical Neurophysiology. 2018 Oct;129(10):2127-2131.

van den Munckhof B, Braun KPJ, Jansen FE. Encefalopathie met elektrische status epilepticus in slaap. Epilepsie, periodiek voor professionals. 2018 Dec.

Jansen FE, van den Munckhof B, Treatment in epileptic encephalopathy with ESES and Landau-Kleffner syndrome. Zeitschrift für Epileptologie. 2019 Jan; 32:135-140.

van den Munckhof B, Zwart AF, Weeke LC, Claessens NHP, Plate JDJ, Leemans A, Kuijf HJ, van Teeseling HC, Leijten FSS, Benders MJN, Braun KPJ, de Vries LS, Jansen FE. Perinatal thalamic injury: MRI predictors of electrical status epilepticus in sleep and long-term neurodevelopment. Neurolmage: Clinical. 2020 Feb 25;26:102227. 
van den Munckhof B, Gefferie SR, van Noort SAM, van Teeseling HC, Schijvens MP, Smit W, Teunissen NW, Plate DJP, Huiskamp GJM, Leijten FSS, Braun KPJ, Jansen FE, Bölsterli BK. Sleep slow wave homeostasis and cognitive functioning in children with electrical status epilepticus in sleep. Sleep. 2020 May 6 (e-publication ahead of print).

van den Munckhof B, Arzimanoglou A, Perucca E, van Teeseling HC, Leijten FSS, Braun KPJ, Jansen FE on behalf of the RESCUE ESES study group. Corticosteroids versus clobazam in epileptic encephalopathy with ESES: a European multicenter randomized controlled clinical trial (RESCUE ESES). Submitted.

* These authors contributed equally 


\section{Curriculum Vitae}

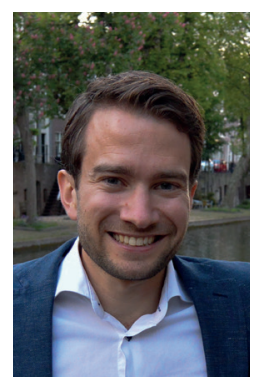

Bart van den Munckhof was born on the $7^{\text {th }}$ of July 1986 in Nijmegen. He graduated cum laude at the Nijmeegse Scholengemeenschap Groenewoud in 2004 and moved to Utrecht to study Medicine in the same year. During his medical studies he did several research projects in internal medicine and neurology and did a tropical medicine internship at Sokoine hospital in Lindi, Tanzania. In 2007/2008 he was president of the board of the Utrecht students sailing association U.S. Histos.

Bart graduated medical school in 2011 and started specialising in neurology at the University Medical Centre Utrecht in 2012. Since 2013 he has combined his neurology training with a PhD programme in paediatric neurology, supervised by dr. Floor E. Jansen and prof. dr. Kees P.J. Braun. He became study coordinator of the European randomised controlled trial RESCUE ESES and completed several other research projects on the aetiology, pathogenesis and treatment of encephalopathy with electrical status epilepticus in sleep (ESES).

During his neurology training and PhD programme, Bart's interest in paediatric neurology grew and he chose to subspecialise in this area. He was a paediatric neurology fellow at the Royal Children's Hospital Melbourne in 2019 / 2020 and started a year of general paediatrics training in 2020. He expects to finalise his specialty training and become a paediatric neurologist in September 2021.

When he is not at work, Bart likes windsurfing, sailing, playing tennis, hiking and camping in the great outdoors. 